\title{
Melt electrospinning towards industrial scale nanofiber production
}

\author{
Citation for published version (APA):
}

König, K. (2020). Melt electrospinning towards industrial scale nanofiber production: an in-depth material and parameter study based on polypropylene and polylactic acid. [Doctoral Thesis, Maastricht University]. ProefschriftMaken. https://doi.org/10.26481/dis.20201203kk

\section{Document status and date:}

Published: 01/01/2020

DOI:

10.26481/dis.20201203kk

Document Version:

Publisher's PDF, also known as Version of record

\section{Please check the document version of this publication:}

- A submitted manuscript is the version of the article upon submission and before peer-review. There can be important differences between the submitted version and the official published version of record.

People interested in the research are advised to contact the author for the final version of the publication, or visit the DOI to the publisher's website.

- The final author version and the galley proof are versions of the publication after peer review.

- The final published version features the final layout of the paper including the volume, issue and page numbers.

Link to publication

\footnotetext{
General rights rights.

- You may freely distribute the URL identifying the publication in the public portal. please follow below link for the End User Agreement:

www.umlib.nl/taverne-license

Take down policy

If you believe that this document breaches copyright please contact us at:

repository@maastrichtuniversity.nl

providing details and we will investigate your claim.
}

Copyright and moral rights for the publications made accessible in the public portal are retained by the authors and/or other copyright owners and it is a condition of accessing publications that users recognise and abide by the legal requirements associated with these

- Users may download and print one copy of any publication from the public portal for the purpose of private study or research.

- You may not further distribute the material or use it for any profit-making activity or commercial gain

If the publication is distributed under the terms of Article $25 \mathrm{fa}$ of the Dutch Copyright Act, indicated by the "Taverne" license above, 
Melt electrospinning towards industrial scale nanofiber production:

An in-depth material and parameter study based on polypropylene and polylactic acid

Kylie Koenig 
Melt electrospinning towards industrial scale nanofiber production:

An in-depth material and parameter study based on polypropylene and polylactic acid Koenig, K.

Maastricht University, 2020

Cover design: @ Sarah Gerling

Printing: ProefschriftMaken (www.proefschriftmaken.nl)

NUR 978

ISBN: 978-94-6423-075-8

(C) Kylie Koenig, Maastricht 2020

All rights reserved. No part of this thesis may be reproduced or transmitted in any form or by any means without prior permission of the author, or when appropriate, of the publisher of the publications. 
Melt electrospinning towards industrial scale nanofiber production:

An in-depth material and parameter study based on polypropylene and polylactic acid

\section{DISSERTATION}

to obtain the degree of Doctor at the Maastricht University, on the authority of the Rector Magnificus,

Prof. dr. Rianne M. Letschert

In accordance with the decision of the Board of Deans, to be defended in public on Thursday $3^{\text {rd }}$ of December 2020 at 10.00 hours

by

Kylie Koenig 
Supervisor

Prof. Dr. G.H. (Gunnar) Seide

\section{Assessment Committee}

Prof. Dr. Pamela Habibovic (Chair)

Prof. Dr. Hans-Josef Endres (Leibniz University Hannover, Germany)

Dr. Jules Harings

Dr.-Ing. Bayram Aslan (TFI Aachen, Germany) 


\section{Abbreviations}

\section{Abbreviations}

$\%(w / w)$

a.R.

AMIBM

ANOVA

DSC

DF

DIN

GPC

HT-SEC

MFI

MFR

PDI

PF

POM

SD

SEM

TGA percentage by weight

after rheometer

Aachen-Maastricht Institute for Biobased Materials

analysis of variance

differential scanning calorimetry

drawn filament

Deutsches Institut für Normung

gel permeation chromatography

high temperature size exclusion chromatography

melt flow index

melt flow rate

polydispersity index

partially oriented filament

polarized optical microscopy

standard deviation

scanning electron microscopy

thermogravimetric analysis

\section{Material abbreviations}
A
compounds containing alizarin
$\mathrm{H}$
compounds containing hematoxylin
HFIP
hexafluor-2-isopropanol
LA
compounds containing alizarin and liquid plasticizer
$\mathrm{LH}$
compounds containing hematoxylin and liquid plasticizer
LQ
compounds containing quercetin and liquid plasticizer
$\mathrm{NaCl}$
sodium chloride
$\mathrm{NaOl}$
sodium oleate 
$\mathrm{NaSt}$

PCL

PES

PLA

PLAG

PP

PU

Q

\section{Physical variables}

F

$\mathrm{F}_{\mathrm{C}}$

$\mathrm{F}_{\mathrm{EF}}$

$F_{G}$

$\ell$

m

$\mathrm{M}_{\mathrm{n}}$

$\mathrm{M}_{\mathrm{w}}$

n

$\mathrm{T}_{\mathrm{cc}}$

$\mathrm{T}_{\mathrm{g}}$

$\mathrm{T}_{\mathrm{m}}$

$X, Y, Z$

$\mathrm{X}_{\mathrm{c}}$

$\Delta \mathrm{A}$

$\Delta \mathrm{W}$

$\dot{\mathrm{Y}}$.

$\dot{Y}_{c}$

$\eta$ sodium stearate

polycaprolactone

Here: viscosity-reducing modified polyester

polylactic acid

poly(lactic-co-glycolic) acid

polypropylene

polyurethane

compounds containing quercetin ratio of the mean-square value for that source of variation to the residual mean square

Coulombic force

electrical field strength

gravitation

distance/length

power law index

number-average molar mass

weight-average molar mass

power law

cold crystallization temperature

glass transition temperature

melting temperature/point

charges in the jet

percentage crystallinity

additional surface area

work to enlarge the surface area

shear rate

critical strain rate

effect strength 


$\begin{array}{ll}\eta^{\prime} & \text { variation of viscosity } \\ \eta^{*} & \text { complex viscosity } \\ \eta_{0} & \text { zero shear viscosity } \\ \sigma & \text { conductivity } \\ \sigma_{S T} & \text { surface tension }\end{array}$




\section{Contents}

Chapter 1 General introduction

A new prototype melt-electrospinning device for the

production of biobased thermoplastic sub-microfibers and nanofibers

Outline of this thesis

Chapter 2 Technical principles of melt electrospinning

Chapter 3 Polypropylene in melt electrospinning

From lab to pilot scale: Melt-electrospun nanofibers of polypropylene with conductive additives

Chapter 4 Polylactic acid in melt electrospinning

The effect of additives and process parameters on the pilot-scale manufacturing of polylactic acid submicrofibers by melt electrospinning

Biobased dyes as conductive additives to reduce the diameter of polylactic acid fibers during melt electrospinning

\section{Chapter 5 Integration of a climate system}

Pilot-scale production of polylactic acid nanofibers by melt electrospinning

Chapter 6 Valorization 


\section{Chapter 1}

\section{General introduction}

A new prototype melt-electrospinning device for the production
of biobased thermoplastic sub-microfibers and nanofibers

K. Koenig, K. Beukenberg, F. Langensiepen, G. Seide

Biomaterials Research. 2019; 23(1):10.

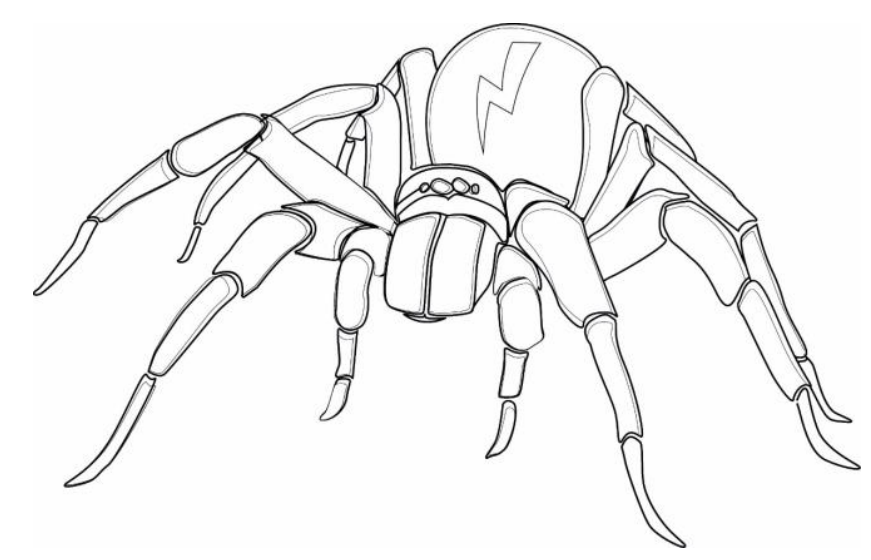




\title{
A new prototype melt-electrospinning device for the production of biobased thermoplastic sub-microfibers and nanofibers
}

\begin{abstract}
Sub-microfibers and nanofibers have a high surface-to-volume ratio, which makes them suitable for diverse applications including environmental remediation and filtration, energy production and storage, electronic and optical sensors, tissue engineering, and drug delivery. However, the use of such materials is limited by the low throughput of established manufacturing technologies. This chapter provides an overview of current production methods for sub-microfibers and nanofibers and then introduces a new meltelectrospinning prototype based on a spinneret with 600 nozzles, thereby providing an important step towards larger-scale production. The prototype features an innovative collector that achieves the optimal spreading of the fiber due to its uneven surface, as well as a polymer inlet that ensures even polymer distribution to all nozzles. We prepared a first generation of biobased fibers with diameters ranging from 1.000 to $7.000 \mu \mathrm{m}$ using polylactic acid and $6 \%(\mathrm{w} / \mathrm{w})$ sodium stearate, but finer fibers could be produced in the future by optimizing the prototype and the composition of the raw materials. Melt electrospinning using the new prototype is a promising method for the production of highquality sub-microfibers and nanofibers.
\end{abstract}

\section{Introduction}

Nanotechnology can be generally defined as the development, handling and control of structures or materials with at least one dimension within the size range 1-100 nm, and the advent of precision tools for nanoscale engineering has promoted great interest in this emerging field over the last 30 years [1, 2]. Nanotechnology exploits the properties of materials that depend on size or structure, particularly properties that differ from the behavior of individual atoms/molecules or larger masses of the same material [2]. The term "nanofiber" is frequently used in the literature to describe very thin fibers without a specified size limit, but a stricter definition as used by the Deutsches Institut für Normung (DIN) standard among others is a structure with two external nanoscale dimensions and a third external dimension that is considerably larger than the nanoscale [3]. However, a comparison of many studies shows that the same term is often used as soon as the fiber diameter falls below $1 \mu \mathrm{m}$ [4]. Although such fibers are not nanofibers according to the DIN standard, the designation has become established and consolidated in recent years. With respect to the DIN standard, another term used to describe fibers with a diameter in the hundreds of nanometers range is "sub-microfiber". 
The small diameter of sub-microfibers and nanofibers provides a high surface-to-volume ratio while maintaining or even improving flexibility compared to conventional fibers. Additionally, many production methods yield porous fibers thus increasing the surface area even further [5]. These properties make such fibers extremely versatile. Their diverse applications include air and water filtration [6, 7], the separation of water/oil and air/oil mixtures [4, 8], technical uses such as the development of lithium-air batteries [912], optical sensors [13] and textiles [5, 14], and medical applications such as tissue engineering [15-23], drug delivery [18, 19, 24-30, 31-36], and the diagnosis and treatment of cancer [27, 36-40] (Figure 1.1).

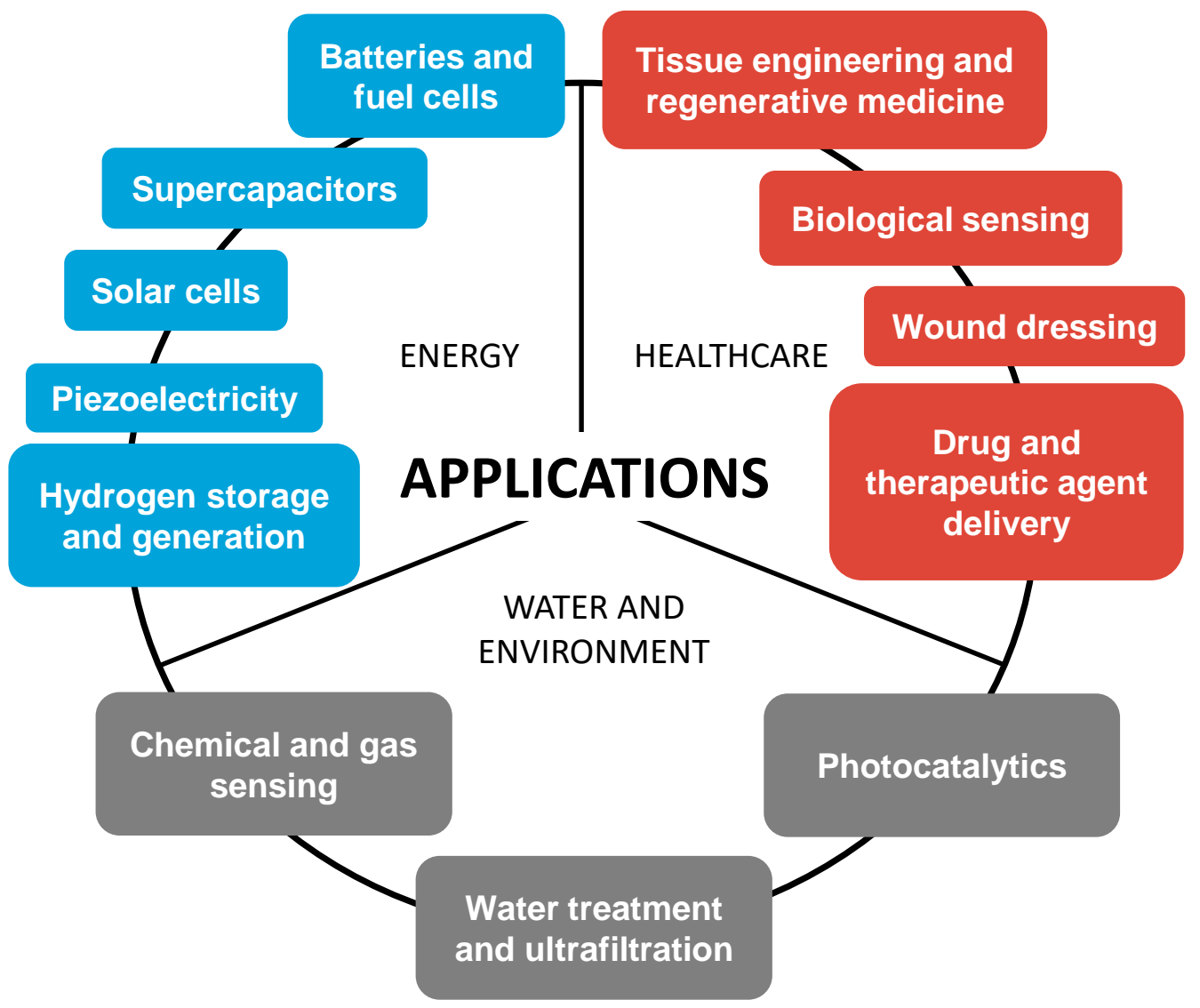

Fig. 1.1: Applications of sub-microfibers and nanofibers [41].

The applications of sub-microfibers and nanofibers depend on their physical and mechanical properties, which in turn depend on the manufacturing process and type of chemistry. This chapter provides an overview of current production methods before describing a novel and scalable melt-electrospinning prototype device and its deployment for the processing of biobased materials into fibers. 


\section{Current manufacturing processes for sub-microfibers and nanofibers}

Sub-microfibers and nanofibers can be produced from a range of biological materials, such as polysaccharides (e.g. chitosan, cellulose, or alginate) and proteins (e.g. gelatin, keratin, or collagen), as well as synthetic polymers, such as polycaprolactone (PCL), polyurethane (PU), polylactic acid (PLA), and poly(lactic-co-glycolic) acid (PLGA). Figure 1.2 provides an overview of current major nanofiber production technologies and the fiber diameters that have typically been achieved using those methods. Only the most common processes are mentioned and there are many variants of these methods that are not discussed in detail [42-50].

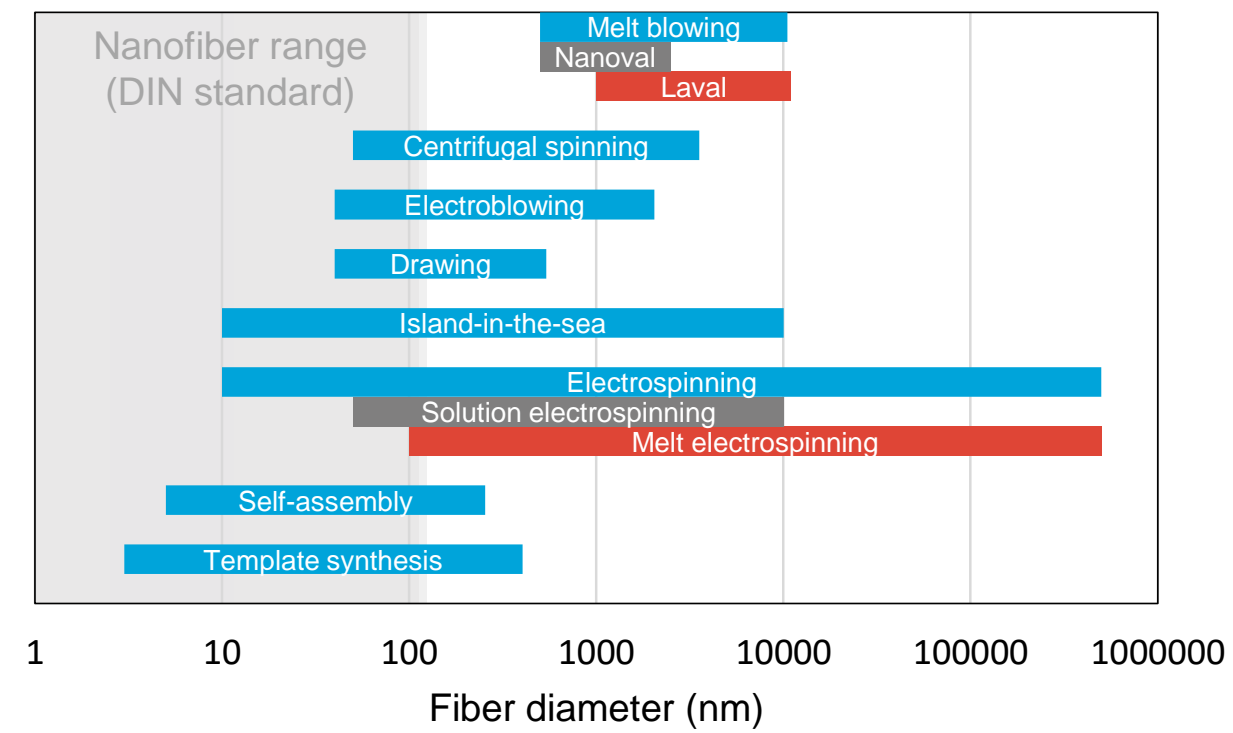

Fig. 1.2: Nanofiber production technologies and the corresponding fiber diameter range: melt blowing [4], nanoval [42, 43], Laval [42], centrifugal spinning [44], electroblowing [45], drawing [46], island-in-the-sea [47], electrospinning [4], solution electrospinning [4], melt electrospinning [4], self-assembly [48, 49], template synthesis [50].

Electrospinning is the most common production method for sub-microfibers and nanofibers, and two fundamental techniques can be distinguished: solution electrospinning and melt electrospinning. Electrospinning combines a strong electrostatic field with the principle of Taylor cone formation. When a droplet of a liquid becomes charged in a field of sufficient strength, the electrostatic repulsion is strong enough to overcome the surface tension and the droplet is stretched. If the charge reaches a certain threshold, a jet erupts from the liquid droplet and this is known as a Taylor cone [51-53]. If the liquid is viscous and cohesive, the jet does not break up into droplets (the principle of electro-spraying) but forms an electrically charged laminar jet, which elongates due to electrostatic repulsion. The jet dries (in the case of solution electrospinning) or cools sufficiently to become solid (in the case of melt electrospinning) 
and a nanoscale fiber is produced [54]. The basic setup for electrospinning is shown in Figure 1.3.

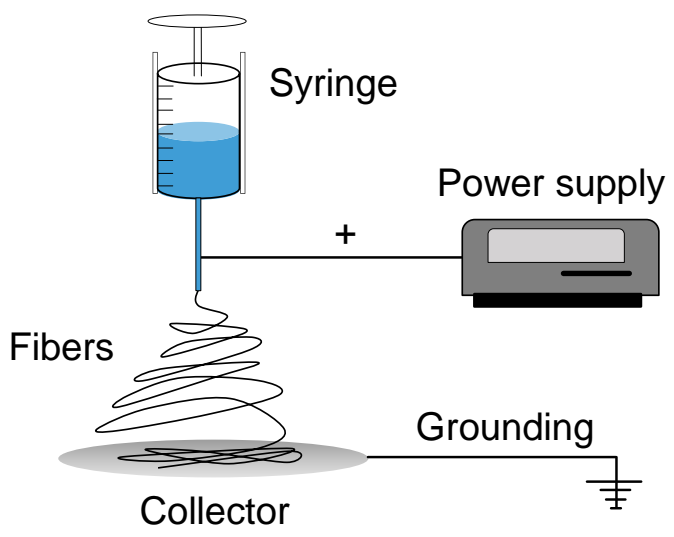

Fig. 1.3: Basic setup of the electrospinning production method.

Solution electrospinning is used more frequently than melt electrospinning for the production of nanofibers because a smaller fiber diameter can be achieved (high hundreds of nanometers), and the equipment has a simpler design and higher productivity compared to current melt-electrospinning devices [55]. The finest fiber produced by melt electrospinning thus far was $80 \mathrm{~nm}$ in diameter [56], although this in not yet routine and typically the fiber diameter is $>2 \mu \mathrm{m}$ [45]. The major advantage of melt electrospinning is that it does not require a solvent, avoiding any risk of toxic solvents being carried over into the mature fiber [57]. Electrospinning is compatible with many different polymers and multiple applications (Table 1.1). 
Tab. 1.1: Overview of electrospinning methods, typical fiber diameters and applications.

\begin{tabular}{|c|c|c|c|c|}
\hline Publication & $\begin{array}{l}\text { Production } \\
\text { method }\end{array}$ & Polymer & $\begin{array}{l}\text { Fiber } \\
\text { diameter } \\
(\mathrm{nm})\end{array}$ & Application \\
\hline Wang 2002 [12] & Solution & $\begin{array}{l}\text { Poly(acrylic } \\
\text { acid)-poly(pyrene } \\
\text { methanol) }\end{array}$ & $100-300$ & $\begin{array}{l}\text { Optical } \\
\text { sensors }\end{array}$ \\
\hline Li 2012 [87] & $\begin{array}{l}\text { Melt (laser } \\
\text { melt) }\end{array}$ & Poly(L-lactic acid) & $2000-7000$ & Biomedical \\
\hline $\begin{array}{l}\text { Dalton } 2006 \\
\text { [89] }\end{array}$ & Melt & $\begin{array}{l}\text { Poly(ethylene } \\
\text { glycol) and } \\
\text { poly(epsilon- } \\
\text { caprolactone) }\end{array}$ & - & - \\
\hline Yoon 2013 [90] & $\begin{array}{l}\text { Melt and S/M- } \\
\text { hybrid }\end{array}$ & $\begin{array}{l}\text { Silk fibroin and } \\
\text { poly(L-lactic acid) }\end{array}$ & $\begin{array}{l}\text { PLA: } 8900 \\
\text { SF: } 820\end{array}$ & $\begin{array}{l}\text { Biomedical } \\
\text { (scaffolding) }\end{array}$ \\
\hline Zhou 2006 [91] & Melt & Poly(L-lactic acid) & & Filtration \\
\hline Kim 2010 & S/M hybrid & $\begin{array}{l}\text { Poly(lactic-co- } \\
\text { glycolic acid) }\end{array}$ & $\begin{array}{l}2800(\mathrm{~S}) \\
530(\mathrm{M})\end{array}$ & $\begin{array}{l}\text { Biomedical } \\
\text { (scaffolding) }\end{array}$ \\
\hline $\begin{array}{l}\text { Scholten } 2011 \\
\text { [7] }\end{array}$ & Solution & Polyurethane & Low 1000s & Air filtration \\
\hline
\end{tabular}

Another common method for nanofiber production is fiber drawing. Here, a solid tip is placed in contact with a liquid polymer and then drawn away, leaving behind a string of polymer liquid that solidifies into a fiber (Figure 1.4).

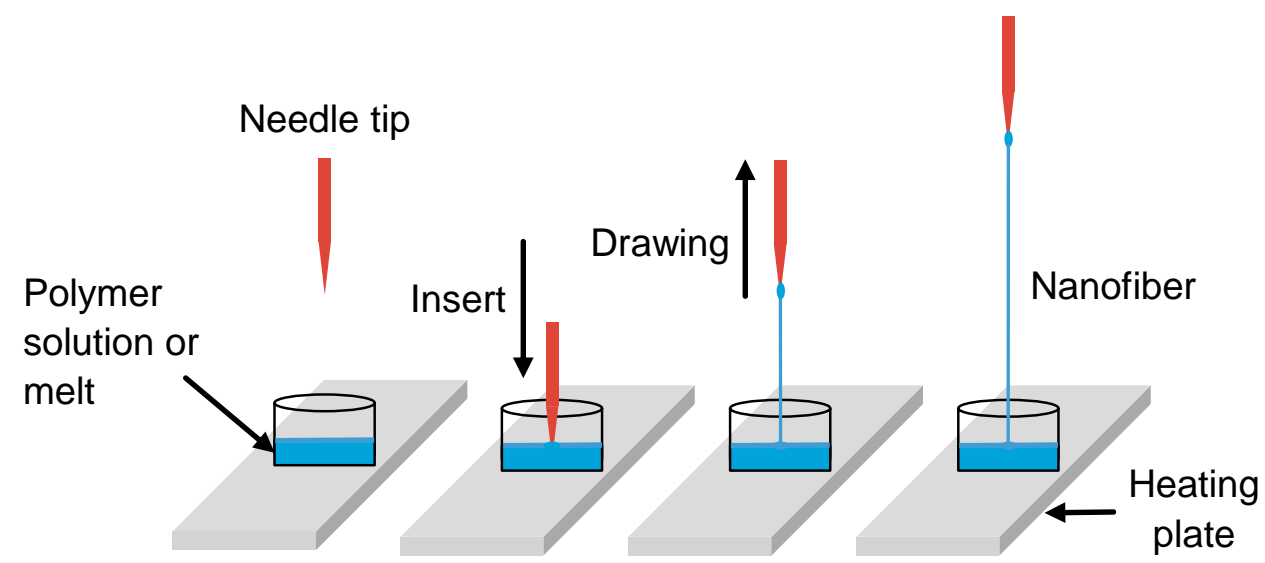

Fig. 1.4: Basic setup of the fiber-drawing production method. 
Like electrospinning, this method is compatible with polymer melts [58] and polymer solutions [46]. One of the main advantages of this method is that it allows the evaluation of single fibers [59]. Drawing typically produces narrower fibers than electrospinning, with diameters of tens of nanometers (Table 1.2).

Tab. 1.2: Overview of non-electrospinning methods, typical fiber diameters and applications.

\begin{tabular}{|c|c|c|c|c|}
\hline Publication & $\begin{array}{l}\text { Production } \\
\text { method }\end{array}$ & Polymer & $\begin{array}{l}\text { Fiber } \\
\text { diameter } \\
(\mathrm{nm})\end{array}$ & Application \\
\hline Xing 2008[58] & Drawing & $\begin{array}{l}\text { Poly(trimethylene } \\
\text { terephthalate) }\end{array}$ & 60 & $\begin{array}{l}\text { Optical } \\
\text { fibers }\end{array}$ \\
\hline Ma 2016 [46] & Drawing & Polyethylene & 40 & - \\
\hline Nakata 2007 [61] & $\begin{array}{l}\text { Island in the } \\
\text { sea }\end{array}$ & $\begin{array}{l}\text { Polyamide } \\
\text { 6/poly(ethylene } \\
\text { terephthalate) }\end{array}$ & 39 & - \\
\hline Uppal 2012 [63] & Melt-blown & & 290 & Filtration \\
\hline Luo [97] & $\begin{array}{l}\text { Template } \\
\text { synthesis }\end{array}$ & $\begin{array}{l}\text { Silver/cross- } \\
\text { linked poly(vinyl } \\
\text { alcohol) }\end{array}$ & Sub-micro & Various \\
\hline Wang 2010 [98] & $\begin{array}{l}\text { Template } \\
\text { synthesis }\end{array}$ & Carbon & 20 & Various \\
\hline Rolandi 2014 [99] & Self-assembly & Chitin & various & Various \\
\hline Xu 2017 [100] & Self-assembly & Polypeptide & & $\begin{array}{l}\text { Drug } \\
\text { delivery }\end{array}$ \\
\hline $\begin{array}{l}\text { Hammami } 2014 \\
\text { [74] }\end{array}$ & Centrifugal & Polyamide 6 & $200-800$ & Various \\
\hline
\end{tabular}

The island-in-the-sea method (Figure 1.5) is a subtype of conventional melt spinning, but two different polymers are involved. One of them (the sea polymer) is spun into a thick fiber within which multiple thinner fibers of the other (the island polymer) are suspended. Following primary extrusion, the sea polymer is removed to leave the nanoscale islandpolymer fibers behind [60]. This method has been used to create polyamide 6/polyethylene terephthalate nanofibers with a consistent diameter of $39 \mathrm{~nm}$ [61]. 


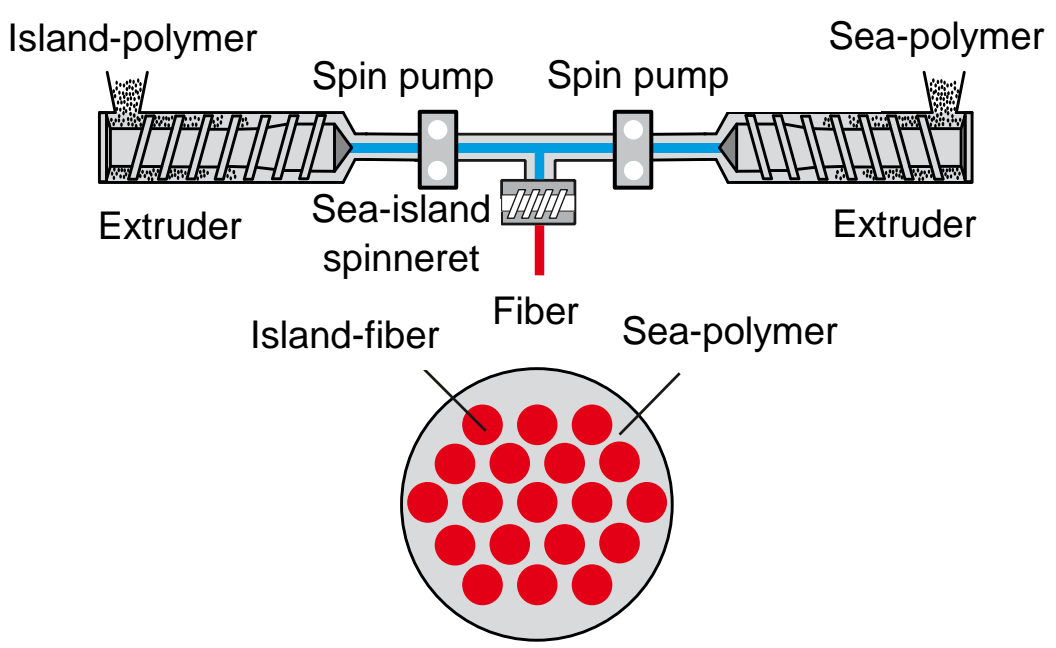

Fig. 1.5: Island-in-the-sea nanofiber production method.

Melt-blown fibers are produced by extruding a polymer melt through small nozzles surrounded by high-speed flowing gas, typically resulting in microfibers approximately $2 \mu \mathrm{m}$ in diameter (Figure 1.6). However, individual sub-microfibers/nanofibers with diameters of $\sim 100 \mathrm{~nm}$ have been produced using an ideal setup comprising an annual air die, Finaplas polypropylene (PP) with a melt flow rate (MFR) of 35 as the polymer, a polymer temperature of $290^{\circ} \mathrm{C}$, a gas temperature of $400^{\circ} \mathrm{C}$ and a feed rate of $4.11 \cdot 10^{-6} \mathrm{~kg} / \mathrm{s}$ [62]. Like electrospinning, which can be scaled up by multiplying the number of jets, melt blowing can be scaled up by multiplying the number of nozzles to reduce costs [63]. However, unlike electrospinning, which can produce aligned fibers, melt-blown fibers are deposited randomly into nonwoven sheets. These are particularly suitable for filtration applications, but melt blowing cannot be used for applications that require oriented fiber sheets.

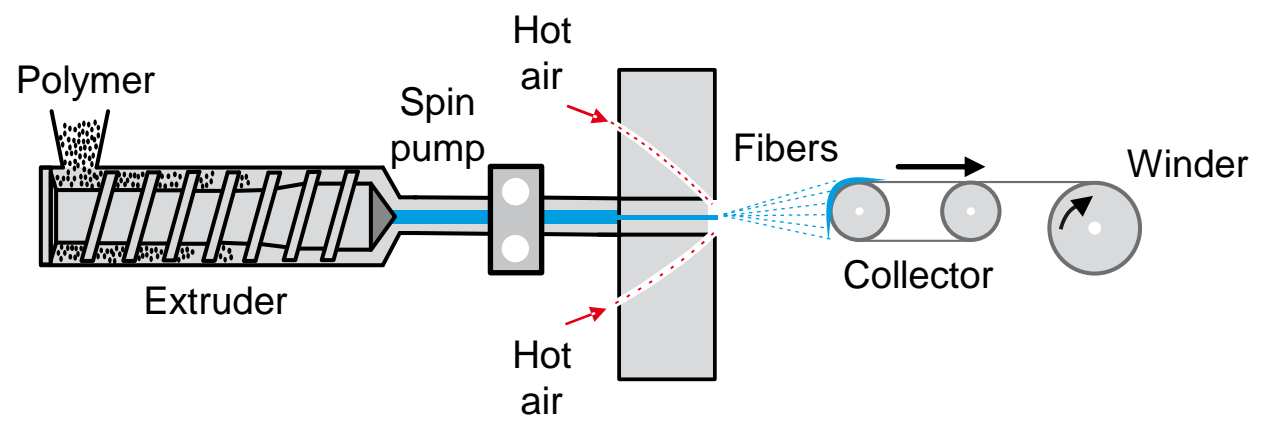

Fig. 1.6: Basic setup of the melt-blowing production method.

A derivative of the melt-blowing technique is the Laval spinning method, which also uses an airstream to draw the fiber from the nozzle [64]. However, the shape of the longitudinal Laval nozzle accelerates the air, making the process more efficient than conventional melt blowing. Furthermore, a cold airstream is used rather than the hot airstream of the conventional method (Figure 1.7). 


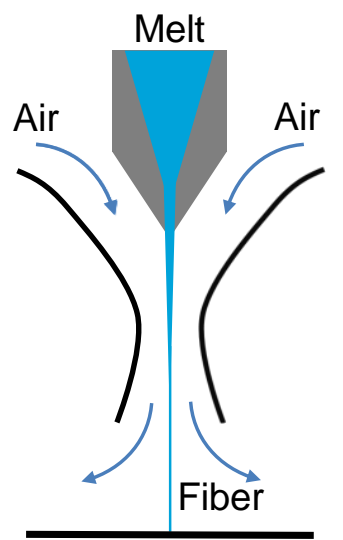

Collector

Fig. 1.7: Airflow through the Laval longitudinal nozzle.

The laminar airstream enters the nozzle from the back. The nozzle narrows just beyond the entrance channel for the polymer, which accelerates the air stream and the fiber to supersonic velocity. The main advantage compared to conventional melt blowing is that the nozzle diameter can be much larger, allowing spinning with a high mass throughput per nozzle [64]. The proprietary Nanoval process is similar to the Laval method but it produces a multitude of smaller-diameter fibers that erupt from the original drawn string when the steadily increasing laminar airflow reaches a particular threshold [65].

Electroblowing is essentially a hybrid of electrospinning and blowing $[64,66]$. The voltage at the spinning nozzle is sufficient to allow Taylor cone formation. The fiber is then caught by a low-velocity airstream that carries it away from the spinneret in a manner similar to conventional melt blowing. However, in contrast to conventional melt blowing, electrostatic repulsion is the main force that pulls the fiber from the nozzle and the purpose of the airflow is to reduce interference from the electric field of adjacent nozzles, making the process easier to scale up [64]. Like electrospinning, electroblowing has two variants: solution electroblowing and melt electroblowing, the latter illustrated in Figure 1.8. In both cases, the airstream also cools down the liquid fiber to solidify it (melt electroblowing) or to dry it and remove the solvent (solution electroblowing).

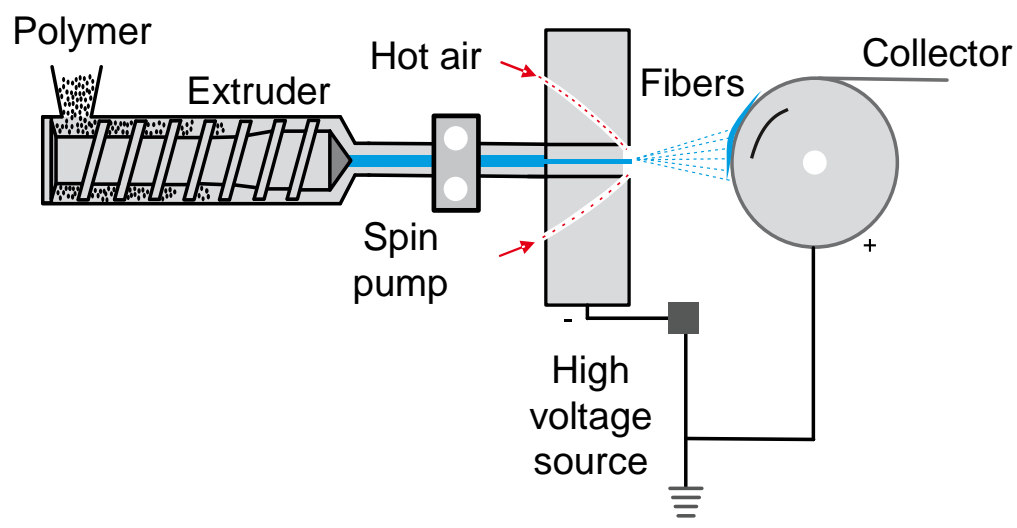

Fig. 1.8: Basic setup of the hybrid melt-electroblowing production method. 
Flash spinning is a special form of solution spinning, in which the solvent is a hydrocarbon such as butane or isobutene, which would exist as gas under normal atmospheric pressure at room temperature. The spinning solution is maintained under very high pressure at temperatures of $130-500^{\circ} \mathrm{C}$. When the spinning solution is ejected into an environment with a much lower pressure and temperature, the fiber dries immediately $[67,68]$.

Other, lesser-used production methods include template synthesis and self-assembly. Template synthesis is suitable for the production of both fibers and tubules [50,69]. It uses the pores of a host material as a template to control the growth of new materials [57]. For example, polymers can be produced electrochemically by applying a metal layer to a membrane with pores within which the polymers are synthesized [57]. Figure 1.9 demonstrates the procedure and shows how the fiber diameter and length are controlled by the pore dimensions. [70]

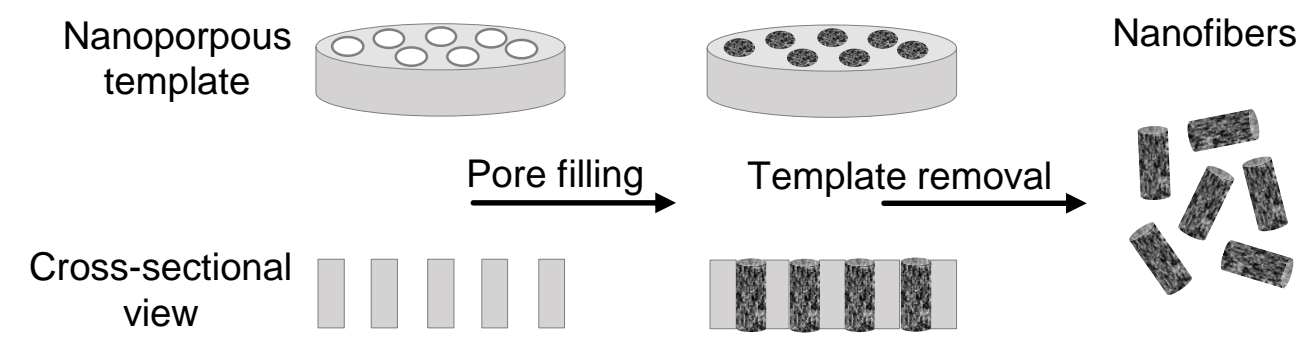

Fig. 1.9: Overview of template synthesis.

Self-assembly is used for the production of nanofibers comprising polypeptides with an intrinsic capacity for self-assembly [71]. The method is based on the spontaneous organization of individual macromolecules into an ordered and stable nanoscale structure [41]. A solution is necessary to create the appropriate environment for the formation of these structures, which have a potential minimum diameter of $3 \mathrm{~nm}$ [72]. Although very small diameters can be achieved, this technology is complex and has a low throughput, making it difficult to scale up and thus unsuitable for industrial applications [41].

Whereas melt blowing and its variations are easy to scale up, these methods cannot produce oriented fibers. In contrast, the other methods can produce oriented fibers but are hampered by their low throughput. Centrifugal spinning can overcome this challenge by mounting the spinneret on a centrifuge with the nozzles facing outward [73, 74]. When the centrifugal force (dependent on the rotor diameter and angular velocity) is sufficient to overcome the drag caused by the viscosity of the polymer solution or melt, a steady polymer jet streams from the nozzle to the collector [73, 74]. The centrifugal spinning method is shown in Figure 1.10. 


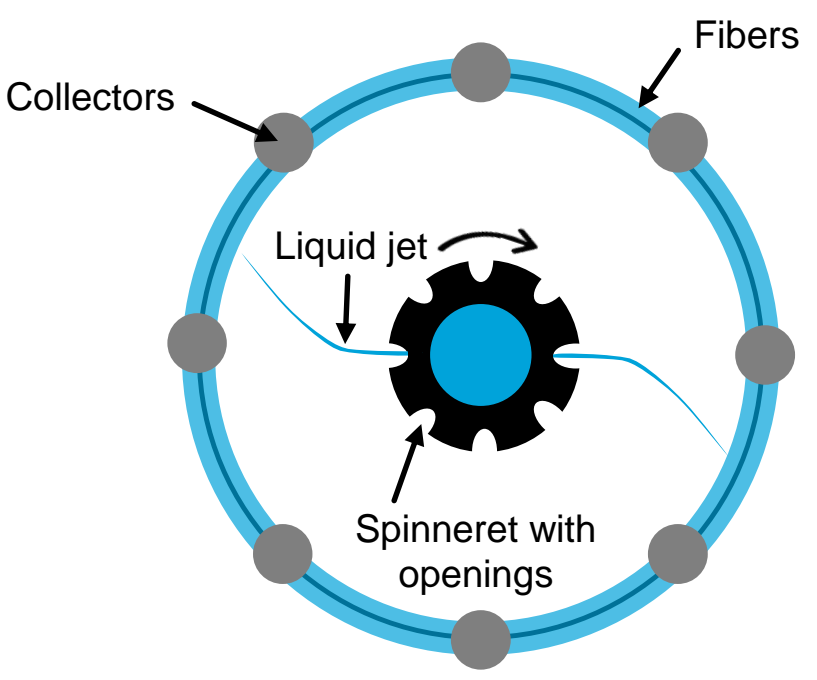

Fig. 1.10: Basic setup of the centrifugal spinning production method.

A derivative of this method is split-fiber production, where the nozzle is split into several smaller nozzles to produce narrower fibers or flat bands [75]. The throughput is up to 500-fold higher than conventional solution electrospinning [76]. However, the use of solvents and the strong dependence on the elasticity of the polymer solution and the evaporation rate of the solvents make this process difficult to control [77].

\section{Development of a new melt-electrospinning prototype for sub- microfibers and nanofibers}

\section{Work leading up to the prototype - state of the art}

Electrospinning methods allow the production of single or multiple nanofibers (depending on the number of jets) using a simple apparatus with relatively low setup and operating costs, so electrospinning can be an economically competitive production method [78]. The presence of solvent in the solution electrospinning process adds an expensive recovery step to the overall manufacturing process, and the potential carryover of toxic solvents or solvents with undesirable optical activity makes solution electrospinning unsuitable for medical and filtration applications or the production of optical sensors. Although the high temperature, high viscosity and low conductivity of the molten polymer is a challenge that must be addressed during melt electrospinning $[79,80]$, the absence of solvent ensures stable jet formation, allowing the direct deposition of micrometer to sub-micrometer range fibers and the reproduction of three-dimensional structures [8185]. Melt electrospinning is not compatible with non-thermoplastic materials, including biological polymers such as collagen, but is ideal for sparingly-soluble polymers such as PP and polyethylene. Other commonly used polymers include PCL, PU, PLA, and PLGA [86-92]. 
In order to produce nanoscale fibers, the polymer delivery rate during melt electrospinning must be significantly lower than during solution electrospinning, which explains the absence of melt electrospinning as an industrial manufacturing method for nanofibers [4]. However, only $2-10 \%$ of the liquid processed during solution electrospinning is the polymer (the rest is solvent that evaporates) whereas $100 \%$ of the processed liquid solidifies into fibers during melt electrospinning, indicating that the industrial use of this method could be achieved by scaling the process up [4]. Accordingly, recent device innovations, such as multiple-needle and needleless configurations, have demonstrated a roadmap to overcome the low throughput of melt electrospinning, typically in the $\mu \mathrm{g} / \mathrm{h}$ range [4]. Prototypes with umbellate nozzles containing 60 spinnerets can achieve maximum product deposition rates of $\sim 36 \mathrm{~g} / \mathrm{h}$ $[93,94]$. The largest multi-nozzle spinning device described in the literature thus far features 64 nozzles [95].

\section{Prototype for the scaled-up melt electrospinning of sub-microfibers and nanofibers}

The Aachen-Maastricht Institute for Biobased Materials (AMIBM) at Maastricht University has cooperated with Fourné Maschinenbau GmbH (Alfter-Impekoven, Germany) and Pötter-Klima Gesellschaft für Nanoheiztechnik mbH (Georgsmarienhütte, Germany) to develop a functional prototype of a melt-electrospinning device featuring a spinneret with 600 nozzles, which vastly exceeds the capabilities of any state-of-the-art technologies. The nozzle plate of this device is shown in Figure 1.11. Each nozzle has a diameter of $0.3 \mathrm{~mm}$ and the nozzles are spaced $8 \mathrm{~mm}$ apart.

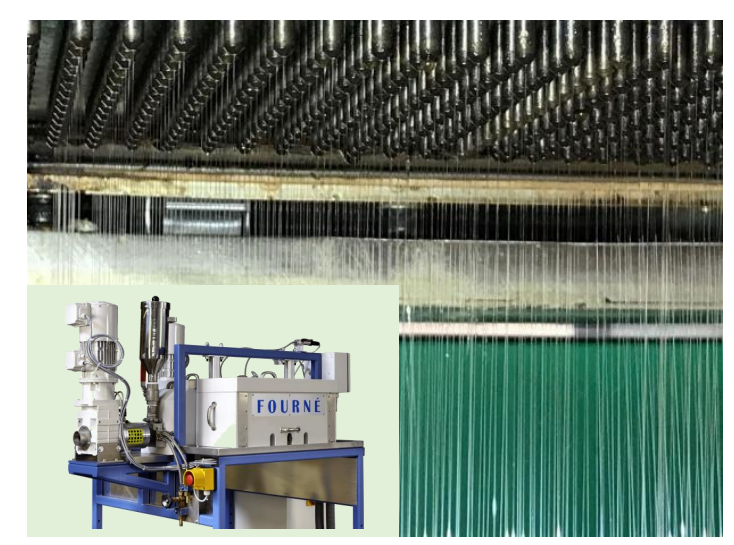

Fig. 1.11: The 600-nozzle melt-electrospinning prototype developed at the AMIBM.

One of the major challenges when scaling up a melt-electrospinning device from a smaller number to a larger number of spinnerets is the uniform distribution of the melt to all nozzles. The low volume flow of the polymer melt during melt electrospinning may lead to incomplete nozzle filling, resulting in sporadic and unpredictable pressure losses within each nozzle. Inside the prototype nozzle, melt flow has been improved by taking this design consideration into account and introducing a three-plate construction and two symmetrically designed polymer inlets. A distributor plate combined with a finely porous 
sintered plate ensures the optimum melt distribution and a uniform pressure build-up over the entire nozzle cross-section. A relatively high specific contact load at the sealing line as well as the use of aluminum flanges guarantees the sealing of the nozzle plates. The constant supply of polymer melt is ensured by a speed-adjustable single-screw extruder and spinning pump.

Another challenge addressed by the new prototype is the tendency for solidified polymer to block the capillaries. The integration of heating elements around the spinneret achieves a uniform polymer melt flow from the nozzles to prevent this common problem during fiber production. A collector with an uneven surface is used instead of a conventional plate collector to facilitate the optimal spreading of the collected fibers (Figure 1.12). With the nozzle/collector pairing installed in the prototype, nonwovens can be produced continuously over a width of $340 \mathrm{~mm}$. The collector is connected to an Eltex KNH65 source supplying a positive high voltage $(1-60 \mathrm{kV})$ while simultaneously grounding the spinneret.

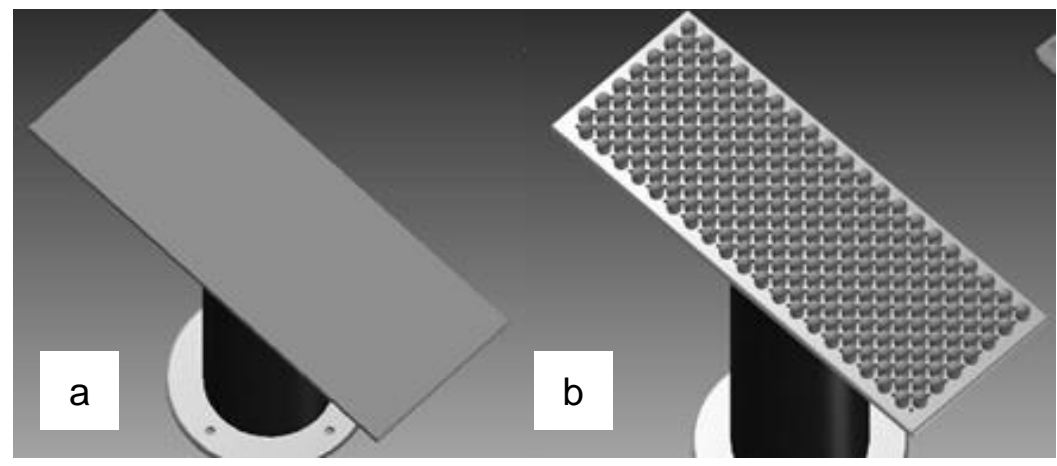

Fig. 1.12: Comparison of (a) a conventional collector and (b) the novel collector designed for the 600-nozzle AMIBM melt-electrospinning prototype.

Initially, the device was used to produce PP fibers containing conductive additives, and the finest fiber had a diameter of $6.64 \mu \mathrm{m}$. This was produced using high-flow PP HL508FB (Borealis AG, Vienna, Austria) containing 2\% (w/w) sodium stearate (Alfa Aesar, Karlsruhe, Germany). The distance between the collector and the nozzle plate was $11 \mathrm{~cm}$, a positive voltage of $60 \mathrm{kV}$ was applied to the collector, the nozzle was heated to $210^{\circ} \mathrm{C}$ and the polymer flow rate was defined by a spinning pump speed of $16 \mathrm{rpm}$ [96]. Having verified the function of the device, we then attempted the production of biobased fibers using Ingeo Biopolymer 6201D, a commercial spinning-grade PLA (NatureWorks LLC, Minnetonka, Minnesota, USA) containing 6\% (w/w) sodium stearate. We maintained the distance between the collector and nozzle plate at $11 \mathrm{~cm}$ but reduced the nozzle temperature to $190^{\circ} \mathrm{C}$ and the spinning pump speed to $2 \mathrm{rpm}$, yielding fibers ranging from 1.000 to $7.000 \mu \mathrm{m}$ in diameter (Figure 1.13). 


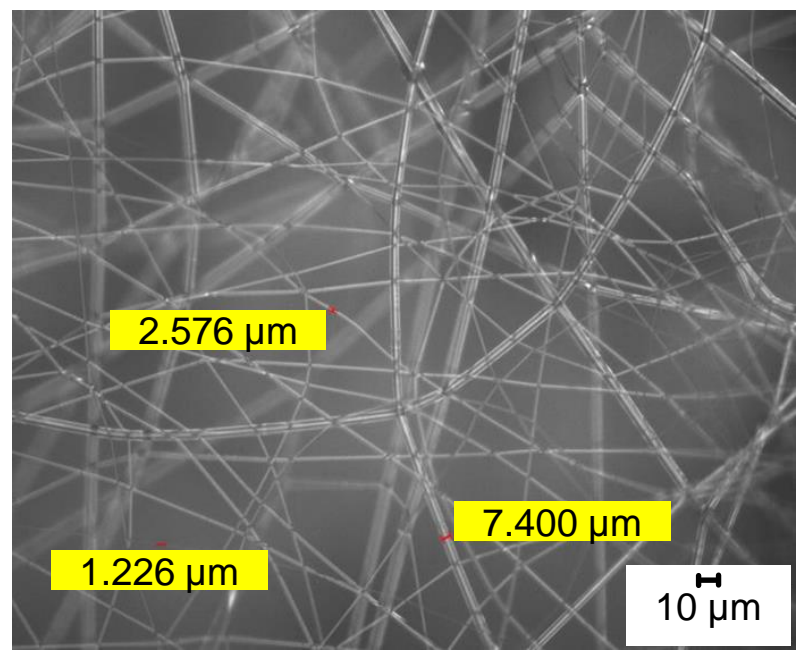

Fig. 1.13: PLA microfibers containing $6 \%(\mathrm{w} / \mathrm{w})$ sodium stearate produced using the 600-nozzle AMIBM melt-electrospinning prototype.

\section{Outlook}

Several methods can be used to produce nanofibers and sub-microfibers, but melt electrospinning is among the most promising technologies in terms of fiber structure and the breadth of downstream applications due to the absence of solvents in the manufacturing process. The major drawback of melt electrospinning is its low throughput, resulting in the adoption of solution electrospinning as the principal industrial process technology. Although some attempts have been made to scale up the electrospinning method, an industrial process has yet to be established. At the AMIBM, a promising, scaled-up melt-electrospinning prototype was developed that bridges the gap between laboratory-scale and pilot-scale manufacturing. Thus far, PLA fibers $\sim 1 \mu \mathrm{m}$ in diameter have been produced, but this was achieved without comprehensive optimization of the apparatus, the process parameters or the polymer substrate and additives. There are many opportunities to improve the performance of the device by adding new features such as a controllable climate chamber around the spinneret to improve jet stretching before the collector, delaying the solidification of the melt and thus producing thinner fibers with uniform diameters. In the future, individually controlled collector tips in a multinozzle structure with the writing ability of melt electrospinning could lead to the development of truly innovative microfiber and nanofiber products.

\section{Acknowledgements}

The authors acknowledge the support of Fourné Maschinenbau GmbH (AlfterImpekoven, Germany), Pötter-Klima Gesellschaft für Nanoheiztechnik mbH (Georgsmarienhütte, Germany), Schnick Systemtechnik $\mathrm{GmbH} \&$ Co KG 
(Heiligenhaus,Germany), and the Chair of Polymer Materials (LSP) of ErlangenNürnberg University (Germany). We also thank our Bachelor's students Nina Goebel and Jaqueline Ellerkmann for their support with the literature research and preparation of figures, and our hands-on technician Henri Becker. 


\section{References}

[1] Back S. The role of nanotechnology in sustainable textiles. In: BLACKBURN, R. S.: Sustainable Textiles. Woodhead Publishing, 2009. 978-1-84569-453-1. p. 302328.

[2] DIN CEN ISO/TS 80004-1: Nanotechnologien - Fachwörterverzeichnis - Teil 1: Kernbegriffe (ISO/TS 80004-1:2015); Deutsche Fassung CEN ISO/TS 80004$1: 2015$.

[3] DIN CEN ISO/TS 80004-2: Nanotechnologien - Fachwörterverzeichnis - Teil 2: Nanoobjekte (ISO/TS 80004-2:2015); Deutsche Fassung CEN ISO/TS 800042:2017.

[4] Brown T, Dalton P, Hutmacher DW, et al. Melt electrospinning today: An opportune time for an emerging polymer process. Elsevier 2016; 56: 116-166.

[5] Bhat GS. Advances in Polymeric Nanofiber Manufacturing Technologies. J Nanomater Mol Nanotechnol 2016; 5: 2324.

[6] Sundarrajan S, Tan KL, Lim SH, et al. Electrospun Nanofibers for Air Filtration Applications. Procedia Eng 2014; 75: 159-163.

[7] Scholten E, Bromberg L, Rutledge GC, et al. Electrospun Polyurethane Fibers for Absorption of Volatile Organic Compounds from Air. ACS Appl Mater Interfaces 2011; 3: 3902-3909.

[8] Sarbatly R, Krishnaiah D, Kamin Z. A review of polymer nanofibres by electrospinning and their application in oil-water separation for cleaning up marine oil spills. Mar Pollut Bull 2016; 106: 8-16.

[9] Song M, Park S, Alamgir F, et al. Nanostructured electrodes for lithium-ion and lithium-air batteries: the latest developments, challenges, and perspectives. Mater Sci Eng R Reports 2011; 72: 203-252.

[10] Wang J, Li Y, Sun X. Challenges and opportunities of nanostructured materials for aprotic rechargeable lithium-air batteries. Nano energy 2013; 2: 443-467.

[11] Zhang G, Zheng J, Liang $\mathrm{R}$, et al. a-MnO2/carbon nanotube/carbon nanofiber composite catalytic air electrodes for rechargeable lithium-air batteries. jes.ecsdl.org 2011; 158: A822.

[12] Truong TT, Liu Y, Ren Y, et al. Morphological and Crystalline Evolution of Nanostructured $\mathrm{MnO}_{2}$ and Its Application in Lithium-Air Batteries. ACS Nano 2012; 6: 8067-8077.

[13] Wang X, Drew C, Lee S-H, et al. Electrospun Nanofibrous Membranes for Highly Sensitive Optical Sensors. Nano Lett 2002; 2: 1273-1275.

[14] Bhardwaj N, Kundu SC. Electrospinning: A fascinating fiber fabrication technique. Biotechnol Adv 2010; 28: 325-347. 
[15] Ma B, Xie J, Jiang J, et al. Rational design of nanofiber scaffolds for orthopedic tissue repair and regeneration. Nanomedicine (Lond) 2013; 8: 1459-81.

[16] Shin S-H, Purevdorj O, Castano O, et al. A short review: Recent advances in electrospinning for bone tissue regeneration. J Tissue Eng 2012; 3:1-9.

[17] McClellan P, Landis WJ. Recent Applications of Coaxial and Emulsion Electrospinning Methods in the Field of Tissue Engineering. Biores Open Access 2016; 5: 212-27.

[18] Cui W, Zhou Y, Chang J. Electrospun nanofibrous materials for tissue engineering and drug delivery. Sci Technol Adv Mater 2010; 11: 014108.

[19] Sill T, von Recum HA. Electrospinning: applications in drug delivery and tissue engineering. Biomaterials 2008; 29 (13): 1989-2006.

[20] Yoo H, Kim TG, Park TG. Surface-functionalized electrospun nanofibers for tissue engineering and drug delivery. Adv Drug Deliv Rev 2009, 61(12): 1033-42.

[21] Khajavi R, Abbasipour M, Bahador A. Electrospun biodegradable nanofibers scaffolds for bone tissue engineering. J Appl Polym Sci 2016; 133: 42883.

[22] Venugopal J, Low S, Choon AT, et al. Interaction of cells and nanofiber scaffolds in tissue engineering. J Biomed Mater Res Part B Appl Biomater 2008; 84(1): 3448.

[23] Vasita R, Katti DS. Nanofibers and their applications in tissue engineering. Int $\mathrm{J}$ Nanomedicine 2006; 1: 15-30.

[24] Chew SY, Wen Y, Dzenis $Y$, et al. The role of electrospinning in the emerging field of nanomedicine. Curr Pharm Des 2006; 12: 4751-70.

[25] Chakraborty S, Liao I-C, Adler A, et al. Electrohydrodynamics: A facile technique to fabricate drug delivery systems. Adv Drug Deliv Rev 2009; 61: 1043-54.

[26] Goyal R, Macri LK, Kaplan HM, et al. Nanoparticles and nanofibers for topical drug delivery. J Control Release 2016; 240: 77-92.

[27] Zamani M, Prabhakaran MP, Ramakrishna S. Advances in drug delivery via electrospun and electrosprayed nanomaterials. Int J Nanomedicine 2013; 8: 29973017.

[28] Weng L, Xie J. Smart electrospun nanofibers for controlled drug release: recent advances and new perspectives. Curr Pharm Des 2015; 21: 1944-59.

[29] Chou S-F, Carson D, Woodrow KA. Current strategies for sustaining drug release from electrospun nanofibers. J Control Release 2015; 220: 584-91.

[30] Zeng J, Xu X, Chen X, et al. Biodegradable electrospun fibers for drug delivery. Elsevier 2003; 92: 227-231. 
[31] Katti DS, Robinson KW, Ko FK, et al. Bioresorbable nanofiber-based systems for wound healing and drug delivery: Optimization of fabrication parameters. J Biomed Mater Res 2004; 70(2): 286-296.

[32] Hu X, Liu S, Zhou G, et al. Electrospinning of polymeric nanofibers for drug delivery applications. Elsevier 2014; 185: 12-21.

[33] Kenawy E, Abdel-Hay, El-Newehy $\mathrm{MH}$, et al. Processing of polymer nanofibers through electrospinning as drug delivery systems. Elsevier 2009; 113: 296-302.

[34] Shen X, Yu D, Zhu L, et al. Electrospun diclofenac sodium loaded Eudragit® L 10055 nanofibers for colon-targeted drug delivery. Int J Pharm 2011; 408: 200-207.

[35] Pillay V, Dott C, Choonara $\mathrm{Y}$, et al. A review of the effect of processing variables on the fabrication of electrospun nanofibers for drug delivery applications. hindawi.com 2013; 2013: 22.

[36] Naves LB, Dhand C, Venugopal JR, et al. Nanotechnology for the treatment of melanoma skin cancer. Prog Biomater 2017; 6: 13-26.

[37] Chen Z, Chen Z, Zhang A, et al. Electrospun nanofibers for cancer diagnosis and therapy. Biomater Sci 2016; 4: 922-932.

[38] Kim Y-J, Ebara M, Aoyagi T. A Smart Hyperthermia Nanofiber with Switchable Drug Release for Inducing Cancer Apoptosis. Adv Funct Mater 2013; 23: 57535761.

[39] Shi D, Bedford NM, Cho H-S. Engineered Multifunctional Nanocarriers for Cancer Diagnosis and Therapeutics. Small 2011; 7: 2549-2567.

[40] Zhang N, Deng Y, Tai Q, et al. Electrospun TiO2 Nanofiber-Based Cell Capture Assay for Detecting Circulating Tumor Cells from Colorectal and Gastric Cancer Patients. Adv Mater 2012; 24: 2756-2760.

[41] Kwnry, Lim CT: Nanofiber technology: current status and emerging developments. In: Topical Volume on Advanced Polymeric Materials Bd. 2017; 70: 1-17.

[42] Blim A, Jarecki L, Blonski S. Modeling of pneumatic melt drawing of polypropylene super-thin fibers in the Laval nozzle. Bull. Pol. Ac. 2014. 62: 42-54.

[43] NANOVAL GMBH \& CO KG: Nanoval - Process. https://www.nanoval.de/verfahren_eng_fasern.htm (accessed 17 Oct 2018).

[44] Badrossamay MR, Mcllwee HA, Goss JA, Parker KK. Nanofiber Assembly by Rotary Jet-Spinning. Nano Lett. 2010; 10(6): 2257-6.

[45] Medeiros ES, Glenn GM, Klamcynski AP, Orts WJ, Mattoso LH. Solution blow spinning: A new method to produce micro- and nanofibers from polymer solutions. Journal of Applied Polymer Science 2009; 113(4): 2322-2330 
[46] Ma J, Zhang Q, Zhang $\mathrm{Y}$, et al. A rapid and simple method to draw polyethylene nanofibers with enhanced thermal conductivity. Appl Phys Lett 2016; 109: 033101.

[47] Masuda M, Funakoshi J. Island-in-sea fiber, combined filament yarn and textile product. EP2821533A1 2012

[48] Biswas A, Bayer IS, Biris AS, Wang T, Dervishi E et al. Advances in top-down and bottom-up surface nanofabrication: Techniques, applications \& future prospects. Advances in Colloid and Interface Science 2012; 170(1): 2-27

[49] Barnes, CP, Sell SA, Boland ED, Simpson DG, Bowlin GL. Nanofiber technology: Designing the next generation of tissue engineering scaffolds. Intersection of Nanoscience and Modern Surface Analytical Methodology 2007; 59 (14): 14131433

[50] Martin CR. Template Synthesis of Electronically Conductive Polymer Nanostructures. Acc Chem Res 1995; 28: 61-68.

[51] Suvorov VG, Zubarev NM. Formation of the Taylor cone on the surface of liquid metal in the presence of an electric field. J Phys D Appl Phys 2004; 37: 289-297.

[52] Suvorov VG, Litvinov EA. Dynamic Taylor cone formation on liquid metal surface: numerical modelling. J Phys D Appl Phys 2000; 33: 1245-1251.

[53] Yarin AL, Koombhongse S, Reneker DH. Taylor cone and jetting from liquid droplets in electrospinning of nanofibers. J Appl Phys 2001; 90: 4836-4846.

[54] Li D, Xia Y. Electrospinning of Nanofibers: Reinventing the Wheel? Adv Mater 2004; 16: 1151-1170.

[55] Ramakrishna S, Fujihara K, Teo W-E, et al. Electrospun nanofibers: solving global issues. Mater Today 2006; 9: 40-50.

[56] Ahn YC, Park SK, Kim GT, Hwang XY, Lee CG et al. Development of high efficiency nanofilters made of nanofibers. Nano Korea 2004 Symposium on NT Challenge 2006; 6(6): 1030-1035

[57] Hutmacher DW, Dalton PD. Melt Electrospinning. Chem - An Asian J 2011; 6: 4456.

[58] Xing $X$, Wang $Y$, Li B. Nanofibers drawing and nanodevices assembly in poly(trimethylene terephthalate). Opt Express 2008; 16: 10815.

[59] Bajakova J, Chaloupek J, et al. „DRAWING“- THE PRODUCTION OF INDIVIDUAL NANOFIBERS BY EXPERIMENTAL METHOD. Nanocon 2011

[60] Ndaro MS, Jin X, Chen T, et al. Splitting of islands-in-the-sea fibers (PA6/COPET) during hydroentangling of nonwovens. J Eng Fiber Fabr 2007; 2: 1-9. 
[61] Nakata K, Fujii K, Ohkoshi Y, et al. Poly(ethylene terephthalate) Nanofibers Made by Sea-Island-Type Conjugated Melt Spinning and Laser-Heated Flow Drawing. Macromol Rapid Commun 2007; 28: 792-795.

[62] Shambaugh RL. A macroscopic view of the melt-blowing process for producing microfibers. Ind Eng Chem Res 1988; 27: 2363-2372.

[63] Uppal R, Bhat G, Eash C, et al. Meltblown nanofiber media for enhanced quality factor. Fibers Polym 2013; 14: 660-668.

[64] Yoon K, Hsiao BS, Chu B. Functional nanofibers for environmental applications. J Mater Chem 2008; 18: 5326-5334.

[65] Gerkin L. Nanoval process for spunbonds detailed. Int Fiber J 2005; 20: 52-56.

[66] Kim YM, Ahn KR, Sung YB, et al. MANUFACTURING DEVICE AND THE METHOD OF PREPARING FOR THE NANOFIBERS VIAELECTRO-BLOWN SPINNING PROCESS. US 7,618,579 B2, USPTO, 2011.

[67] Palmer L. Flash spinning. US3565979A, USPTO, 1968.

[68] Nayak R, Padhye R, Kyratzis IL, et al. Recent advances in nanofibre fabrication techniques. Text Res J 2012; 82: 129-147.

[69] Martin CR. Nanomaterials: A Membrane-Based Synthetic Approach. Science 1994; 266: 1961-1966.

[70] Karatas A, Algan AH. KARATAŞ.Template Synthesis of Tubular Nanostructures for Loading Biologically Active Molecules. Current topics in medicinal chemistry $2016 ; 17$.

[71] Malkar NB, Lauer-Fields JL, Juska D, et al. Characterization of Peptide-Amphiphiles Possessing Cellular Activation Sequences. Biomacromolecules 2003; 4: 518-528.

[72] Hassanzadeh $P$, Kharaziha M, Nikkhah M, Shin SR, Jin J et al. Chitin nanofiber micropatterned flexible substrates for tissue engineering. Journal of Materials Chemistry 2013; 1(34): 4217-4224.

[73] Zhang $X$, Lu Y. Centrifugal spinning: An alternative approach to fabricate nanofibers at high speed and low cost. Polym Rev 2014; 54: 677-701.

[74] Hammami MA, Krifa M, Harzallah O. Centrifugal force spinning of PA6 nanofibers - processability and morphology of solution-spun fibers. J Text Inst 2014; 105: 637647.

[75] Peno E, Lipton R, Kay S. Split fiber producing devices and methods for the production of microfibers and nanofibers. US8778240B2, USPTO, 2012. 
[76] Ren L, Ozisik R, Kotha SP. Rapid and efficient fabrication of multilevel structured silica micro-/nanofibers by centrifugal jet spinning. Journal of Colloid and Interface Science 2014; 425: 136-142.

[77] Ren L, Ozisik R, Kotha SP, Underhill P. Highly Efficient Fabrication of Polymer Nanofiber Assembly by Centrifugal Jet Spinning: Process and Characterization. Macromolecules 2015; 48 (8): 2593-2602.

[78] Garg K, Bowlin GL. Electrospinning jets and nanofibrous structures. Biomicrofluidics 2011; 5: 13403.

[79] Nayak R. Polypropylene Nanofibers: Melt Electrospinning Versus Meltblowing. Engineering Material. Springer International Publishing 2017

[80] Bubakir M, Barhoum A, Li H, Yang W. Handbook of Nanofibers (1st edtn), Springer Nature. 2017.

[81] Willerth SM. Electrospun Materials for Tissue Engineering and Biomedical Applications: Research, Design and Commercialization. Woodhead Publishing. 2017.

[82] Wunner FM, Wille ML, Nanoon TG, Bas O, Dalton PD et al. Melt Electrospinning Writing of Highly Ordered Large Volume Scaffold Architectures. Adv. Mater. 2018; 30:1706570.

[83] Wunner FM, Maartens J, Bas O, Gottschalk K, De-Juan-Pardo E et al. Electrospinning writing with molten poly ( $\varepsilon$-caprolactone) from different directions - Examining the effects of gravity. Material Letters 2018; 216: 114-118.

[84] Eichholz KF, Hoey DA. Mediating human stem cell behaviour via defined fibrous architectures by melt electrospinning writing. Acta Biomaterialia 2018; 75: 140-151.

[85] Dayan CB, Afghah F, Okan BS, Yıldız M, Menceloglu Y et al. Modeling 3D melt electrospinning writing by response surface methodology. Materials \& Design 2018; 148: 87-95.

[86] Zhao F, Liu Y, Yuan H, et al. Orthogonal design study on factors affecting the degradation of polylactic acid fibers of melt electrospinning. J Appl Polym Sci 2012; 125: 2652-2658.

[87] Li X, Liu H, Liu J, et al. Preparation and experimental parameters analysis of laser melt electrospun poly(L-lactide) fibers via orthogonal design. Polym Eng Sci 2012; 52: 1964-1967.

[88] Larrondo L, St. John Manley R. Electrostatic fiber spinning from polymer melts. I. Experimental observations on fiber formation and properties. J Polym Sci Polym Phys Ed 1981; 19: 909-920.

[89] Dalton PD, Lleixà Calvet J, Mourran A, et al. Melt electrospinning of poly-(ethylene

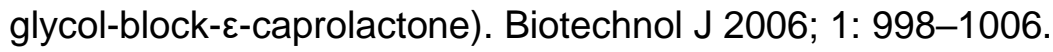


[90] Yoon Y II, Park KE, Lee SJ, et al. Fabrication of microfibrous and nano/microfibrous scaffolds: melt and hybrid electrospinning and surface modification of poly(L-lactic acid) with plasticizer. Biomed Res Int 2013; 2013: 309048.

[91] Zhou H, Green TB, Joo YL. The thermal effects on electrospinning of polylactic acid melts. Polymer (Guildf) 2006; 47: 7497-7505.

[92] Brown TD, Dalton PD, Hutmacher DW. Direct Writing By Way of Melt Electrospinning. Adv Mater 2011; 23: 5651-5657.

[93] Li H, Chen $\mathrm{H}$, Zhong $\mathrm{X}$, et al. Interjet distance in needleless melt differential electrospinning with umbellate nozzles. J Appl Polym Sci 2014; 131: 40515.

[94] Liu Y, Zhao F, Zhang C, Zhang J, Yang W. Solvent-free preparation of poly(lactic acid) fibers by melt electrospinning using an umbrella-like spray head and alleviation of the problematic thermal degradation. J. Serb. Chem. Soc 2012; 77:1071-1082.

[95] Hacker CJP, Seide G, Gries T, Thomas H, Moeller M. Electrospinning of polymer melt: steps toward an upscaled multi-jet process. In:Proc Int Confer Latest Advances in High Tech Textiles and Textile-Based Materials 2019: 71-6.

[96] Koenig K, Daenicke J, Langensiepen F, Seide G, Schubert DW. From Lab to Pilot Scale: Melt Electrospun Nanofibers of Polypropylene with Conductive Additives. J Nanomater Mol Nanotechnol 2019; 8:1.

[97] Luo L-B, Yu S-H, Qian H-S, et al. Large-scale synthesis of flexible gold/crosslinked-PVA sub-microcables and cross-linked-PVA tubes/fibers by using templating approaches based on silver/cross-linked-PVA sub-microcables. Chemistry 2006; 12: 3320-4.

[98] Wang $\mathrm{Y}$, Zheng M, Lu H, et al. Template synthesis of carbon nanofibers containing linear mesocage arrays. Nanoscale Res Lett 2010; 5: 913-6.

[99] Rolandi M, Rolandi R. Self-assembled chitin nanofibers and applications. Adv Colloid Interface Sci 2014; 207: 216-222.

[100] Xu D, Samways DSK, Dong H. Fabrication of self-assembling nanofibers with optimal cell uptake and therapeutic delivery efficacy. Bioact Mater 2017; 2: 260268. 


\section{Chapter 1}

\section{General introduction}

Outline of this thesis

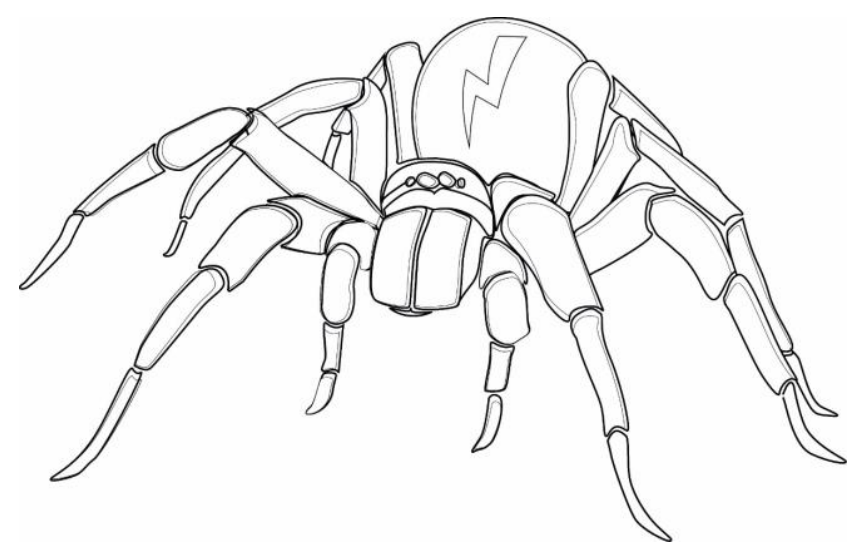




\section{Outline of this thesis}

The aim of this thesis is to develop a melt-electrospinning process for the production of nanofibers based on a novel, scaled-up, melt-electrospinning prototype through commissioning and further development of the device as well as targeted material modification.

In Chapter 1, the new developed scaled-up melt-electrospinning prototype featuring 600 nozzles, bridging the gap between laboratory-scale and industrial-scale nanofiber manufacturing, is presented. Herewith tackling the industry-relevant key challenge of increasing the production of nanofibers for various applications such as filtration and medicine in a sustainable and efficient way.

Chapter 2 focuses on the technical principles of melt electrospinning. This is intended to identify the main factors influencing the fiber formation, such as the forces acting on the polymer melt, process parameters and different electrospinning setups. The first preconditions are set, therefore the influence of the parameters, polymer flow rate and temperature on the fiber diameter as well as material modifications resulting in an increased electrical polymer conductivity and/or decreased polymer viscosity, can be investigated and validated.

In Chapter 3, the feasibility of fabricating polypropylene nanofibers is investigated using the conductive additives sodium stearate, sodium oleate and Irgastat during melt electrospinning with a single-nozzle lab-scale and the 600-nozzle pilot-scale device. A comparison is made between the two devices with regard to the effects on the fiber diameter and the material behavior.

In Chapter 4, the manufacturing of biobased polylactic acid sub-microfibers by melt electrospinning using a single-nozzle laboratory-scale and the novel 600-nozzle pilotscale device combined with the conductive and viscosity-reducing additives: sodium stearate, sodium chloride and a polyester-based plasticizer is investigated. Additionally, a two-way variance analysis is performed to determine the influence of the independent parameters spin pump speed, temperature and additive concentration on the fiber diameter. Since the added salts can potentially increase the hydrolysis and degradation of the moisture-sensitive PLA, the use of the biobased dyes alizarin, hematoxylin and quercetin as conductivity-enhancing additives and a biobased plasticizer in laboratoryscale melt electrospinning is also investigated.

In Chapter 5, the effects on the fiber diameter by the integration of a climate control system in form of a glass chamber are investigated, thus aiming for a nanofiber production comparable to the more conventional solution-electrospinning process. The factors, viscosity behavior of the melt-electrospun materials at different temperatures and spin pump speed, are again taken into account. 
Chapter 6 deals with the economic relevance of an upscaled melt-electrospinning process, classifies the novel process into existing production processes and gives first product examples for which the process can be a sustainable substitute.

Chapter 7 provides an overview of the results achieved and an outlook on possible further development steps. Further material modification possibilities as well as plantspecific developments are pointed out.

The initial situation, objectives, methods and key results are summarized in Figure 1.14.

\section{Initial situation}

- Solution electrospinning is the most commonly used process for the production of nanofibers 4 Drawback: Toxicity of solvents, expensive solvent recovery step

- Alternative eco-friendly process: Melt electrospinning

4 Drawback: No industrial process available, low electrical polymer conductivity and higher viscosity results in lager fiber diameters, complex device design due to high temperatures

\section{Aim}

- Industrial upscaling of the melt-electrospinning process

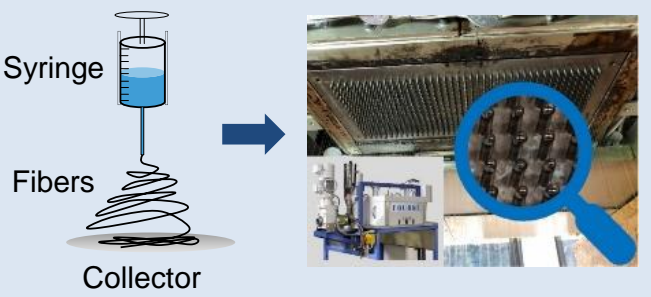

Single nozzle device $\quad$ 600-nozzle device

- Tailoring the fiber diameter via process, material and device optimization

- Obtaining a process comparable to the solventelectrospinning process with average fiber diameters below $1 \mu \mathrm{m}$

\section{Development steps}

\section{- Process optimization}

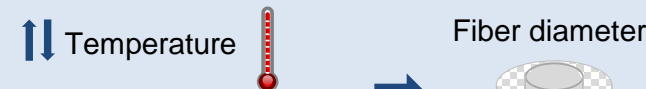

\\Flow rate

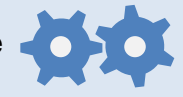

- Material modification

$$
\text { Melt electrospinning }
$$

PP \& PLA

Fiber testing

V. Viscosity-reducing, conductivity-increasing additives

- Device design

Adjustable pin collector

- Integration of a climate system

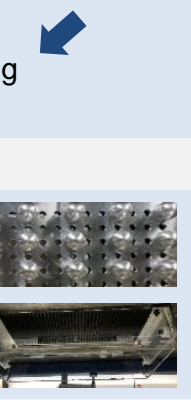

\section{Outcome}

- An average fiber diameter of $810 \mathrm{~nm}$ is achieved using PLA Ingeo Biopolymer 6252, and the finest individual fiber $(420 \mathrm{~nm}$ in diameter) was produced at a spin pump speed of $5 \mathrm{rpm}(1.6 \mathrm{~cm} 3)$ and spinneret set temperature of $230^{\circ} \mathrm{C}$

- The fiber diameters achieved are the smallest fiber diameters yet achieved with an upscaled multi-nozzle melt-electrospinning device of this size

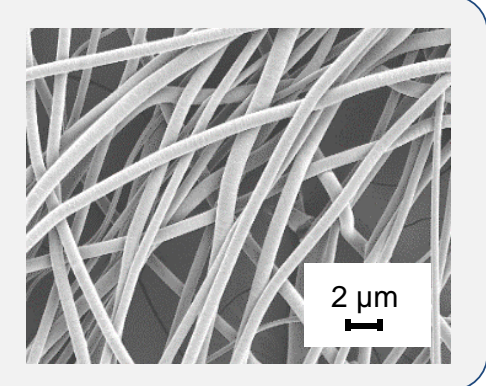

Fig. 1.14: Outline of this thesis. 



\section{Chapter 2}

Technical principles of melt electrospinning

State of the art

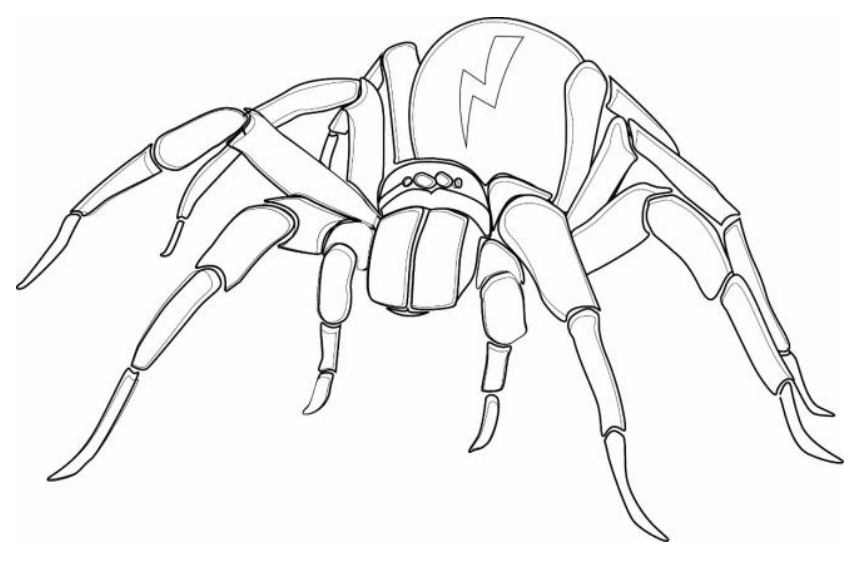




\section{Technical principles of melt electrospinning}

For a stable fiber spinning process, it is important to understand the complex physical and chemical interrelations of melt electrospinning in detail. Chapter $\mathbf{2}$ focuses on the forces acting on the polymer melt, process parameters and electrospinning setups influencing the fiber formation.

\section{Physical and chemical principles of melt electrospinning}

When an electrical potential is applied to the polymer melt, the melt is electrostatically charged and a drop with a defined curvature, the so-called Taylor cone, is formed at the tip of the nozzle. The Taylor cone is a phenomenon caused by the equilibrium state on the surface of the polymer melt between electrostatic Coulombic forces and surface tension forces. While the surface tension of the fluid prefers spherical shapes with minimal surface area due to an energetically favorable state, Coulombic forces promote large surface areas [1]. Only at a certain applied voltage, at which the electrostatic repulsion of the charges on the droplet surface exceeds the surface tension, a continuous jet is formed [2]. Figure 2.1 depicts the different forces having an impact on the formation of the jet when it is electrostatically charged [3].

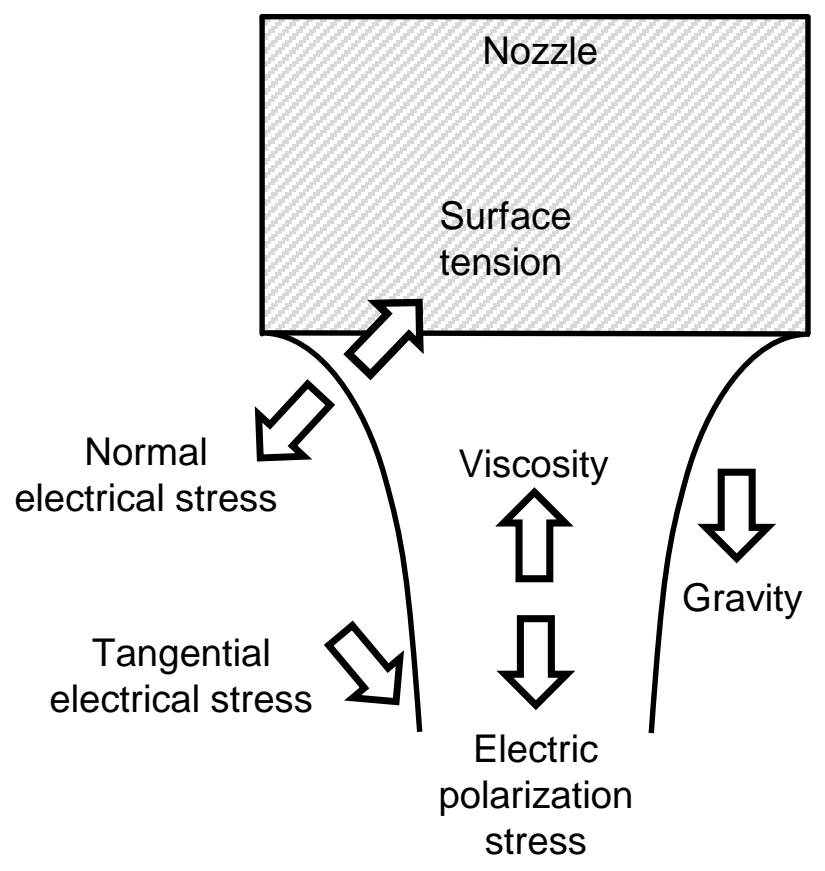

Fig. 2.1: Forces acting on the jet when it is electrically charged.

The surface tension and the viscosity of the polymer melt are acting against the electrical stress. This is expressed in the formula of surface tension $\sigma_{\mathrm{ST}}$ :

$$
\sigma_{\mathrm{ST}}=\frac{\Delta \mathrm{W}}{\Delta \mathrm{A}}
$$

The surface tension $\sigma_{\mathrm{ST}}$ is defined as the work $\Delta \mathrm{W}$ that has to be done to enlarge the surface, divided by the additional surface area $\Delta \mathrm{A}$ [4]. Thus, a higher surface tension 
requires a higher electrical voltage/work to overcome the existing attraction forces between the molecules and build a new surface area. A higher melt viscosity impedes the cone formation and thus reduces the surface area, leading to a higher surface tension. In contrast to this, gravity and the electrical polarization stress accelerate the formation of a polymer jet $[3,5]$.

Once a jet has been formed, various instabilities act and influence the jet during the flight. Those instabilities - axisymmetric Rayleigh instability, axisymmetric conducting instability and non-axisymmetric whipping instability - are the dominant factors for the stretching and thinning of the jet [6]. As those factors are called instabilities, they can influence the jet in favor of building a fiber or working against it. For instance, the Rayleigh instability describes the breakup of the polymer jet into multiple droplets with the aim of reducing the free surface energy [7]. This phenomenon is used and wanted for the corresponding electrospraying process in which the droplets are desired. In contrast to melt electrospinning, the occurrence of Rayleigh instability must be suppressed. This instability, driven by surface tension, can mainly be prevented by an additional elongational stress from an external electric field and a higher surface charge density of the polymer melt [6].

The other two instabilities are electrically driven and are independent of the surface tension in comparison to the Rayleigh instability [6]. The axisymmetric conducting instability leads to an elongation and thinning of the jet along the axis by Coulombic forces and the repulsion of adjacent charges along the polymer jet [1]. Until then, the jet remains symmetrically arranged along the axis. Further away from the nozzle a nonaxisymmetric bending instability occurs, which leads to whipping [1, 8]. According to previous publications, the whipping has a significant influence on the fiber diameter [6]. Reneker et al. [9] have reported different mathematical and physical phenomena that are responsible for the bending instability and the whipping. Firstly, the electrical charges of the jet can be considered as a static system of charges, which mainly interact by Coulombic forces, unless an additional external field is present. According to Earnshaw's theorem, all magnetic and electric fields are known to be unstable, i.e. there is a small displacement or disturbance that interferes with the stability of the system. In Figure 2.2 the phenomenon of bending/whipping is illustrated. 


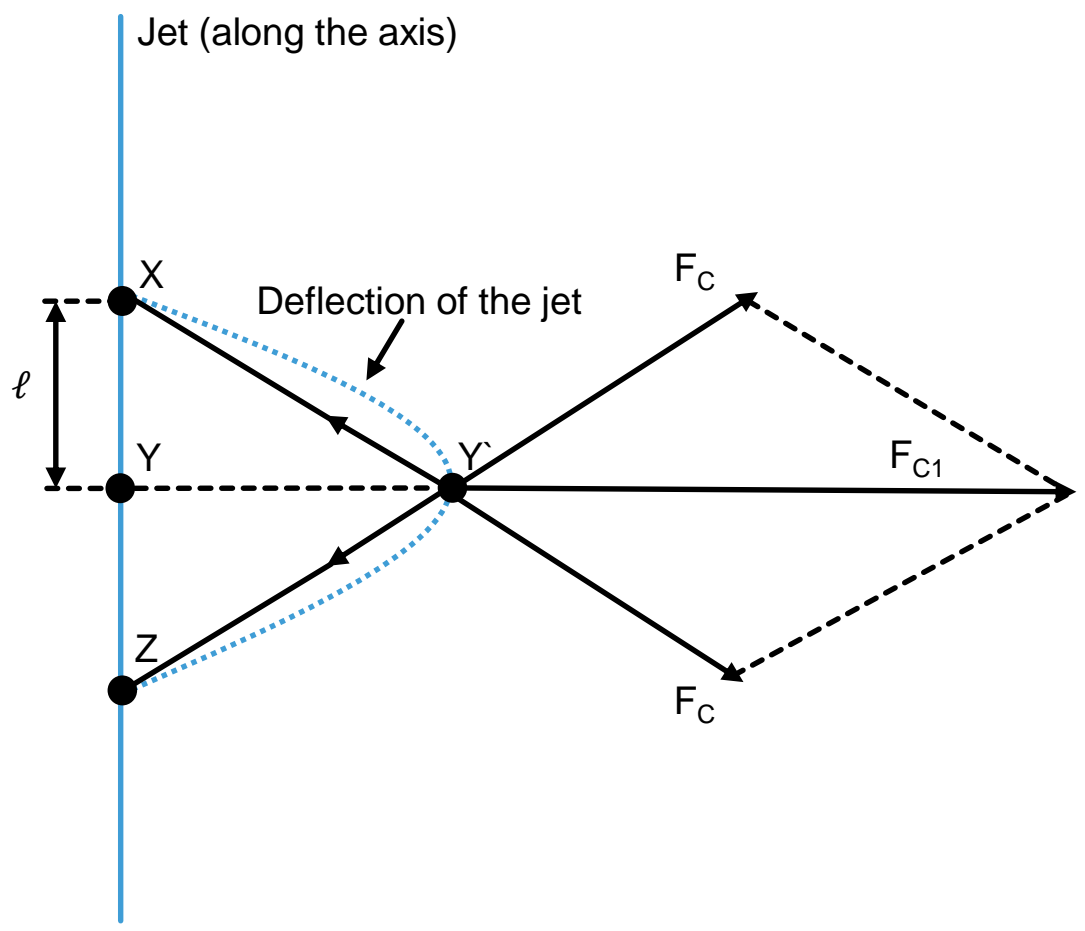

Fig. 2.2: Bending instability.

Three adjacent equal charges $(X, Y, Z)$ are aligned on a common axis and are oriented in the polymer jet. Due to a random displacement of the charge $Y$ towards $Y^{\prime}$, two Coulombic forces $\left(F_{C}\right)$ acting against $Y^{\prime}$ resulting in the force $F_{C 1}$ which pulls the charge $Y^{\prime}$ further away from the axis. Consequently, the bending increases with a growing curvature of the jet [9]. The bending causes an elongation of the distance, which the jet has to travel until it reaches the collector, resulting in higher stretching and therewith thinner fiber diameters are achieved [1]. In addition, the polymer has more time to solidify [8]. These present physical principals depend to a certain degree on the used parameter settings. According to Bubakir et al. [10], the non-axisymmetric whipping instability can only be observed at low melt viscosities and is far less pronounced in melt electrospinning than in solution electrospinning.

\section{Correlation of melt-electrospinning parameters}

In the following the impact and interrelationships of three parameter groups - material, process and environmental parameters - relevant for the melt-electrospinning process are discussed. The fundamental parameters for melt electrospinning are given in Table 2.1. 
Tab. 2.1: Fundamental parameters in melt electrospinning.

\begin{tabular}{|l|l|}
\hline \multicolumn{2}{|c|}{ Material parameters } \\
\hline Polymer properties & Melt characteristics \\
\hline Molecular weight & Conductivity \\
\hline Molecular weight distribution & Viscosity \\
\hline Tacticity & Surface tension \\
\hline Secondary valences & Crystallization \\
\hline
\end{tabular}

\begin{tabular}{|l|l|}
\hline Process parameters & Environmental parameters \\
\hline Temperature & Air humidity \\
\hline Electrical field strength & Ambient air temperature \\
\hline Electrical voltage & Air velocity \\
\hline Flow rate & \\
\hline Nozzle diameter & \\
\hline Distance spinneret to collector & \\
\hline
\end{tabular}

Due to the complex correlation of the parameters, many publications analyze the influence of those on the final fiber properties [11-14]. Although lots of research has been done and is still ongoing, not all the resulting effects, especially the impact of the environmental parameters, are yet fully understood [15]. An overview of the most important relations and their consequences on the fiber diameter is given below.

As already discussed, whipping has a high impact on the fiber diameter. Without the presence of this instability, the jet is drawn in a straight line, preventing elongation and thus stretching of the jet. This has an enormous influence on the mechanical properties of the fiber as well as on the fiber diameter. Thus, the parameters primarily influencing the whipping instability are shown in Table 2.2. In addition, the effects of decreasing or increasing the parameters that influence the whipping motion with the aim of reducing the fiber diameter are given and explained in the following. 
Tab. 2.2: Parameters influencing whipping instability.

\begin{tabular}{|l|l|}
\hline \multicolumn{2}{|c|}{ Whipping } \\
\hline Material parameters & Process parameters \\
\hline Molecular weight & Temperature \\
\hline Conductivity & Electrical field strength \\
\hline Viscosity & Flow rate \\
\hline & Distance spinneret to collector \\
\hline
\end{tabular}

Parameter change: Increase $\uparrow$ Decrease

Effect: First positive, then negative

\section{Material parameters}

It is known that the molecular weight is a crucial parameter influencing viscosity during melt electrospinning and thus the formation of the jet. At high molecular weights, the viscosity is higher, which requires higher electrical forces to form a jet. On the other hand, if the molecular weight is too low the jet will break and no continuous fiber will be produced.[16] This also explains the impact on the whipping motion. With a high molecular weight, the whipping is not so strong because higher forces are necessary to initiate the whipping. With a higher conductivity of the polymer the whipping is supported due to a higher charge repulsion [17]. The same phenomenon occurs with a lower viscosity. The molecular motion increases with decreasing viscosity, which also leads to greater charge repulsion and whipping [18]. However, due to high viscosity and low conductivity of the polymer melt in melt electrospinning, the whipping is not always observed, which leads to thicker fiber diameters than in solution electrospinning [19].

\section{Process parameters}

Higher melt temperatures decrease the viscosity of the polymer melt and simultaneously the conductivity is increased. The electrical conductivity increases, since the mobility of charge carriers increases with increasing temperature. Therefore, heating zones along the spinning zone are a good opportunity to regulate the process [14]. Furthermore, the electrical field strength and the throughput adjustable via the flow rate and nozzle diameter are important parameters to guarantee a stabilized spinning process [6]. On the one hand, the electrical field strength must be strong enough to form a jet and to induce the bending instability. On the other hand, if the electrical voltage is too high the whipping is suppressed because the speed of the jet increases and there is not enough time to start the whipping process [20]. To overcome this obstacle, a higher flow rate is required. However, if the flow rate is too high compared to the electrical voltage, the 
amount of supplied material increases, resulting in an increased fiber diameter [12]. Accordingly, it is essential to find the correct setting for these two parameters.

The distance between the nozzle and the collector creates space for the whipping motion, leading to thinner fiber diameters with increased distance. At the same time the electrical field strength decreases with an increase of the distance, and therefore effects the whipping negatively [21].

\section{Environmental parameters}

Ambient temperature above the melting temperature of the polymer between the nozzle and collector prevents the jet from solidifying, increases the whipping motion and therewith the elongation of the jet due to a lower viscosity, resulting in a reduced fiber diameter. Therefore, climate control systems are already being used in small-scale meltelectrospinning devices $[14,19]$. In addition, the relative humidity, which is usually $65 \%$ when testing textiles, must be significantly lower, otherwise negative effects such as voltage discharge can occur [11].

\section{Melt electrospinning setups}

An important factor in melt electrospinning is a high voltage supply for the formation of continuous fibers. The functionality of the process requires two poles between which an electric field can be formed. In many melt-electrospinning devices, either the nozzle or the collector is statically charged (positively or negatively) while the opponent is grounded [22]. Only a few studies report that both machine parts are charged [23]. Figure 2.3 shows the three different possibilities for generating an electrical field [22]. 
a)

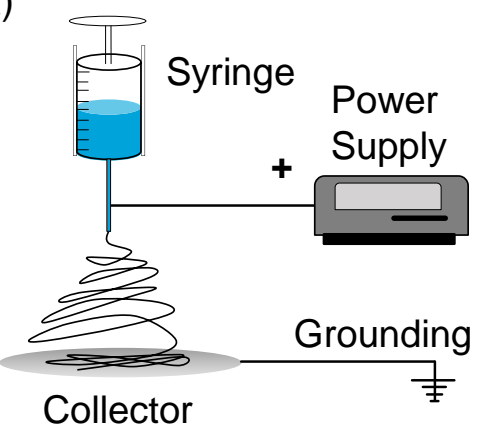

b)

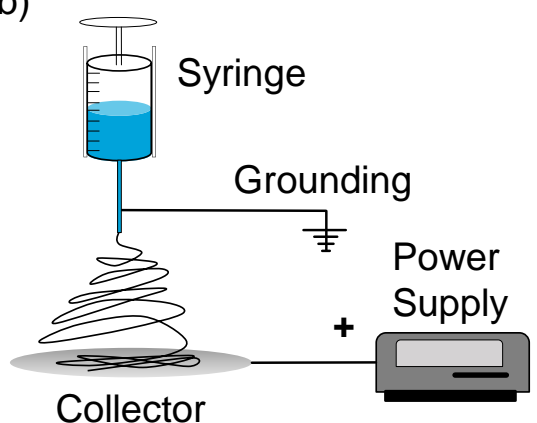

Collector

c)

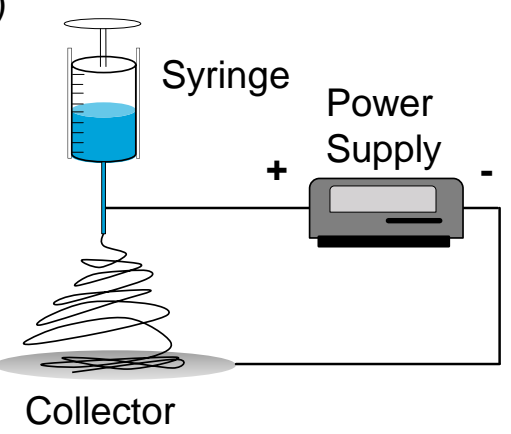

Fig. 2.3: Melt-electrospinning set up: a) syringe charged, collector grounded;

b) syringe grounded, collector charged; c) syringe and collector charged.

According to Brown et al. [11], charging the jet leads to the build-up of electrical charges on machine parts and electrical equipment, e.g. an integrated extruder, and consequently to irreversible damage due to the risk of flashovers. Therefore, the nozzle must be shielded to prevent damage to other machine parts. Alternatively, the collector is statically charged and the nozzle and adjacent machine parts are grounded. Investigations of Kilic et al. [22] show that changing the poles result in a different process efficiency and fiber diameters. Reasons are different electrical forces, resulting from the sum of the Coulombic force and the electric field strength. While the electric field strength $\left(F_{E F}\right)$ and the gravitation $\left(F_{G}\right)$ is equal for both setups, the polarization of the polymer melt and correspondingly the Coulombic forces $\left(F_{C}\right)$ differ, shown in Figure 2.4 [22]. 
a)

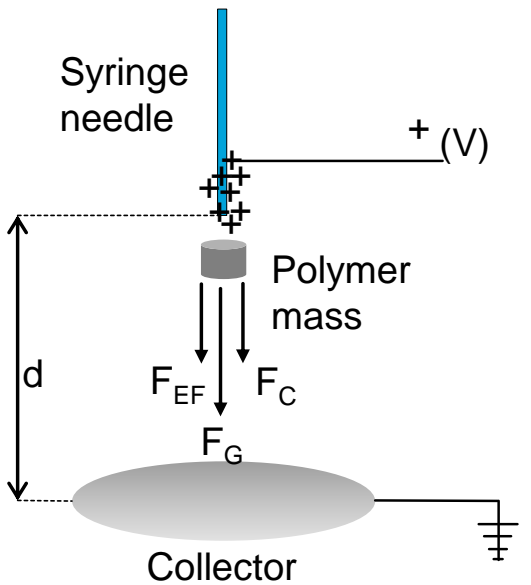

b)

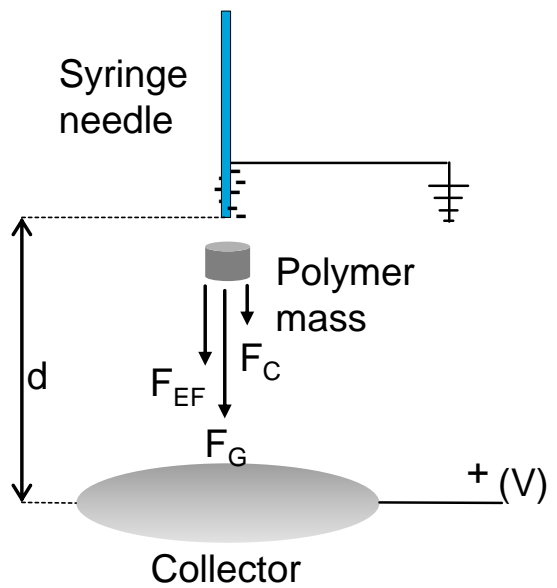

Fig. 2.4: Electrical forces acting on the jet: a) syringe charged, collector grounded, b) syringe grounded, collector charged.

When charging the collector instead of the nozzle, the polymer melt is now charged via the gap between the collector and the nozzle. The dielectric properties of the polymer melt resist the charge resulting in the difference of the electrical forces. Furthermore, the surface area of the collector is significantly larger compared to the nozzle, reducing the charge density for a given applied voltage. [11]. Accordingly, charging the collector leads to a thicker fiber diameter or, in the case of polymers with extremely low conductivity, a jet cannot be formed. Those effects cause a very low process efficiency [22]. In this case, only the increase of the high voltage empowers the formation of the jet.

Overall, it is obvious that the existing machine configuration plays an important role and influences the fiber diameter. Depending on the available machine configuration, the power supply needs to be adjusted. In addition, as the conductivity of the polymer increases, the lower electric field caused by charging the collector can be compensated.

\section{Preconditions for this thesis}

As described above, when integrating an extruder into the melt-electrospinning setup, it must be ensured that the electrical components are protected from the high voltage applied. Therefore, in all attempts described hereafter, the collector of the pilot-scale melt-electrospinning device is positively charged and the spinneret is grounded (Fig. 2.4b). The distance between the collector and the spinneret $(11 \mathrm{~cm})$, as well as the applied voltage $(60 \mathrm{kV})$ are kept constant. Thus, the influence of the parameters, polymer flow rate and temperature on the fiber diameter as well as material modifications resulting in an increased electrical polymer conductivity and/or decreased polymer viscosity, can be investigated and validated.

In Chapter $\mathbf{3}$ and Chapter $\mathbf{4}$, the materials polypropylene and polylactic acid used in this study and their significance for the melt-electrospinning process are described. 


\section{References}

[1] Reneker DH, Yarin AL. Electrospinning jets and polymer nanofibers. Polymer. 2008; 49(10):2387-425.

[2] Rayleigh L. XX. On the equilibrium of liquid conducting masses charged with electricity. The London, Edinburgh, and Dublin Philosophical Magazine and Journal of Science. 1882; 14(87):184-6.

[3] Hartman R, Brunner D, Camelot D, Marijnissen J, Scarlett B. Elecytrohydrodynamic Atomization in the Cone-Jet Mode Physical Modeling of the Liquid Cone and Jet. Journal of Aerosol Science. 1999; 30(7):823-49.

[4] Oberflächenspannung - Lexikon der Physik: Spektrum Akademischer Verlag; https://www.spektrum.de/lexikon/physik/oberflaechenspannung/10567 (accessed 21 September 2018).

[5] Shin YM, Hohman MM, Brenner MP, Rutledge GC. Experimental characterization of electrospinning: the electrically forced jet and instabilities. Polymer. 2001; 42(25):09955-67.

[6] Shin YM, Hohman MM, Brenner MP, Rutledge GC. Electrospinning: A whipping fluid jet generates submicron polymer fibers. Applied Physics Letters. 2001; 78(8):1149-51.

[7] Wang M, Hsieh AJ, Rutledge GC. Electrospinning of poly(MMA-co-MAA) copolymers and their layered silicate nanocomposites for improved thermal properties. Polymer. 2005; 46(10):3407-18.

[8] Kim G-M. Verstärkungsmechanismen auf Makro-, Mikro- und Nano-Längenskalen in heterogenen Polymerwerkstoffen [Habilitation]: Martin-Luther-Universität HalleWittenberg; 2007.

[9] Reneker DH, Yarin AL, Fong H, Koombhongse S. Bending instability of electrically charged liquid jets of polymer solutions in electrospinning. Journal of Applied Physics. 2000; 87(9):4531-47.

[10] Bubakir M, Li H, Yang W. Advances in Melt Electrospinning Technique. 2017. p. $1-30$.

[11] Brown T, Dalton P, Hutmacher DW. Melt electrospinning today: an opportune time for an emerging polymer process. Elsevier. 2016; 56.

[12] Doustgani A, Ahmadi E. Melt electrospinning process optimization of polylactic acid nanofibers. Journal of Industrial Textiles. 2016; 45(4):626-34.

[13] Hassounah I. Melt Electrospinning of Thermoplastic Polymers: RWTH Aachen University; 2012.

[14] Zhou H, Green TB, Joo YL. The thermal effects on electrospinning of polylactic acid melts. Polymer (Guildf). 2006; 47. 
[15] von Bistram M. Strukturierte funktionelle Nanofasern durch Elektrospinnen: Philipps-Universität Marburg 2007.

[16] Lyons J, Li C, Ko F. Melt-electrospinning part I: processing parameters and geometric properties. Polymer. 2004; 45(22):7597-603.

[17] Nayak R, Kyratzis IL, Truong YB, Padhye R, Arnold L. Melt-electrospinning of polypropylene with conductive additives. Journal of Materials Science. 2012; 47(17):6387-96.

[18] Mit-uppatham C, Nithitanakul M, Supaphol P. Ultrafine Electrospun Polyamide-6 Fibers: Effect of Solution Conditions on Morphology and Average Fiber Diameter. Macromolecular Chemistry and Physics. 2004; 205(17):2327-38.

[19] Koenig K, Daenicke J, Langensiepen F, Seide G, Schubert DW. From lab to pilot scale: melt electrospun nanofibers of polypropylene with conductive additives. $J$ Nanomater Mol Nanotechnol. 2019; 8(2).

[20] Hao MF, Liu Y, He XT, Ding YM, Yang WM. Factors Influencing Diameter of Polypropylene Fiber in Melt Electrospinning. Advanced Materials Research. 2011; 221:129-34.

[21] Deng R, Liu Y, Ding Y, Xie P, Luo L, Yang W. Melt Electrospinning of Low-Density Polyethylene Having a Low-Melt Flow Index. Journal of Applied Polymer Science. 2009; 114:166-75.

[22] Kilic A, Oruc F, Demir A. Effects of Polarity on Electrospinning Process. Textile Research Journal - TEXT RES J. 2008; 78:532-9. 



\section{Chapter 3}

Polypropylene in melt electrospinning

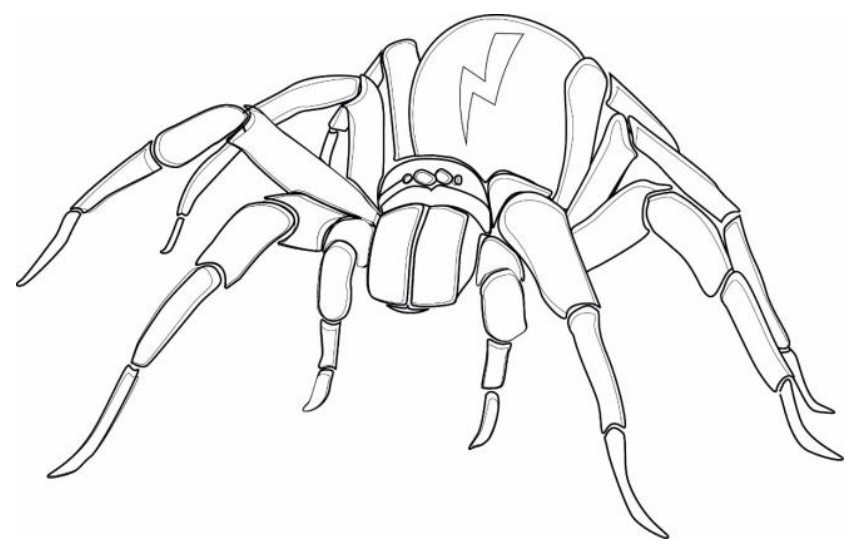




\section{Polypropylene in melt electrospinning}

In the following, we will take a closer look at the importance of polypropylene in the textile industry and explain how it found its way to melt electrospinning.

Polypropylene is the first stereoregular polymer to have achieved industrial importance. The fibers from polypropylene were introduced in the textile sector in the 1970s, have become an important member of the rapidly growing family of synthetic fibers, and rank themselves directly after the three main classes of fibers, polyester, polyamide and polyacrylic. Polypropylene staple fibers are mainly used as nonwovens for geotextiles, filter fabrics, in disposable goods like wipes and diapers, hygiene products, insulation, construction as well as carpets [1]. The size of the global polypropylene nonwovens market was estimated at USD 21.39 billion in 2018 and is expected to register a compound annual growth rate of $6.5 \%$ over the forecast period until 2025 [2]

When the melt is extruded or spun, it retains the key characteristic of polypropylene [3]:

- Total absence of hydrophilicity

- Lower specific weight among all fibers. The density of polypropylene ranges from 0.895 to $0.92 \mathrm{~g} / \mathrm{cm}^{3}$, thus it is $34 \%$ lighter than polyester and $20 \%$ lighter than nylon.

- Outstanding resistance to non-oxidizing bases and acids

- Insolubility in common solvents

- Totally resistant to mold and bacteria

- Abrasion resistance

- Lowest thermal conductivity of any natural or synthetic fiber

Due to these special material properties and the fact that polypropylene is not soluble in most organic solvents at room temperature (soluble in xylene, tetralin and decalin at higher temperatures) and thus cannot easily be used in the solvent electrospinning process, the polymer was the first polymer used for the melt-electrospinning process in 1981 by Larrondo and Manley [4]. They demonstrated that continuous filaments of rapidly crystallizing polymers, such as polypropylene, can be spun from the melt using an electric field as the only driving force. The fiber diameters obtained ranged from $20-$ $180 \mu \mathrm{m}$. Polypropylene is known to be a good electrical insulator and is therefore nonconductive. Furthermore, the viscosities in melt electrospinning are between 20$500 \mathrm{~Pa} \cdot \mathrm{s}$ depending on the polymer and shear rate used, which is much higher compared to solvent electrospinning with viscosities between 5-20 Pa.s [5]. These two circumstances make it challenging to use polypropylene in its initial state, if small fiber diameters in the nanometer range are desired, using the melt-electrospinning process, so that various studies have been carried out on the use of additives to improve processability. Two additive groups are described hereafter. On the one hand, salts or other conductive additives are used to increase the conductivity of polymer melts, which usually have no or low polarity, and on the other hand plasticizers, which reduce the viscosity of polymer melts $[6,7]$. 


\section{Conductive additives}

To increase the polarity and conductivity of the polymer melt, sodium oleate, stearic acid, sodium stearate and sodium chloride were used in various studies [7-10]. All additives have reduced the fiber diameter due to higher charge repulsion in the jet and stronger induced whipping instability. However, the effect of the additives is different. Stearic acid, which is a fatty acid and compared to the other additives not a salt, has a lower polarization effect on the polymer melt. According to Liu et al. [11], salts can easily be ionized in an electric field in which the positive and negative ions move to opposite ends and generate an electric force along the direction of the electric field. In contrast, in stearic acid, under a strong electric field, only a shift occurs in the electron cloud, which leads to a much lower polarization. However, Nayak et al. [7] have investigated that the use of sodium chloride and sodium oleate increases the viscosity of the polymer melt, which is undesirable at first sight as low viscosities are required for melt electrospinning. Despite the higher viscosity, the fiber diameter could be reduced by the additional and stronger conductivity. Sodium chloride has a greater influence on the diameter, since the smaller radius of the ions and the higher mobility of the ions (compared to sodium oleat) lead to a higher charge density and thus to a higher charge repulsion. In comparison to this, Malakhov et al. [9] have used sodium stearate, which decreased the viscosity.

\section{Viscosity-reducing additives}

Another possibility to reduce the fiber diameter of melt-electrospun fibers is the use of viscosity-reducing additives, so called plasticizers. Plasticizers are small molecules compared to the polymer chains and they reduce the secondary binding forces by penetrating into the free volume around the polymer chains. In addition, the intermolecular distance increases, resulting in a larger free volume. These two phenomena lead to higher chain mobility and lower viscosity. With a lower viscosity, smaller fiber diameters are achieved as the polymer molecules can move more easily and thus fewer forces are required to stretch and elongate the jet [12]. The best quality polypropylene fibers, with regard to homogeneity and small diameter deviation, have been electrospun at viscosities of 30 to $55 \mathrm{~Pa} \cdot \mathrm{s}$ [6]. However, the thinnest fiber diameters achieved in this study have not reached the nanometer range compared to the use of salts (conductive additives), with which the fiber diameter could be reduced to the nanometer range [8].

In Chapter 3, the feasibility of fabricating polypropylene nanofibers is investigated using the conductive additives sodium stearate, sodium oleate and the antistatic agent Irgastat during melt electrospinning with a single-nozzle lab-scale and the 600-nozzle pilot-scale device. A comparison is made between the two devices with regard to the effects on the fiber diameter and the material behavior. 


\section{References}

[1] Reneker DH, Yarin AL. Electrospinning jets and polymer nanofibers. Polymer. 2008; 49(10):2387-425.

[1] The Fiber Year Consulting. The Fiber Year 2018 - World Survey on Textiles \& Nonwovens. 2018.

[2] Grand View Research. Polypropylene Nonwoven Fabric Market-Industry Report 2025. 2019. https://www.grandviewresearch.com/industry-analysis/polypropylenenonwoven-fabric-market (accessed 21 February 2020).

[3] Nilak. Polypropylene in the textile industry. http://www.nilak.it/en/polypropylene-inthe-textile-industry.html (accessed 21 February 2020).

[4] Larrondo L, St. John Manley R. Electrostatic fiber spinning from polymer melts. I. Experimental observations on fiber formation and properties. Journal of Polymer Science: Polymer Physics Edition. 1981; 19(6):909-20.

[5] Bubakir M, Li H, Yang W. Advances in Melt Electrospinning Technique. 2017. p. 1-30.

[6] Dalton PD, Grafahrend D, Klinkhammer K, Klee D, Möller M. Electrospinning of polymer melts: Phenomenological observations. Polymer. 2007; 48(23):6823-33.

[7] Nayak R, Kyratzis IL, Truong YB, Padhye R, Arnold L. Melt-electrospinning of polypropylene with conductive additives. Journal of Materials Science. 2012; 47(17):6387-96.

[8] Nayak R, Kyratzis IL, Truong YB, Padhye R, Arnold L, Peeters G, et al. Fabrication and Characterisation of Nanofibres by Meltblowing and Melt Electrospinning. Advanced Materials Research. 2012; 472-475:1294-9.

[9] Malakhov SN, Khomenko AY, Belousov SI, Prazdnichnyi AM, Chvalun SN, Shepelev AD, et al. Method of manufacturing nonwovens by electrospinning from polymer melts. Fibre Chemistry. 2009; 41(6):355-9.

[10] Nayak R. Polypropylene nanofibers: melt electrospinning versus Meltblowing. Engineering Material: Springer International Publishing; 2017.

[11] Liu Y, Chen Z, Jianyun H, Zhao F, Liu Y. Effect of polar additives on melt electrospinning of nonpolar polypropylene. Journal of the Serbian Chemical Society. 2014;79:587-96.

[12] Polymer Engineering. How do Plasticizers function? https://www.engtips.com/viewthread.cfm?qid=95589 (accessed 18 November 2018). 


\section{Chapter 3}

\section{Polypropylene in melt electrospinning}

From lab to pilot scale: Melt-electrospun nanofibers of polypropylene with conductive additives

K. Koenig, D. Daenicke, F. Langensiepen, D. Schubert, G. Seide Journal of Nanomaterials \& Molecular Nanotechnology. 2019; 8(2).

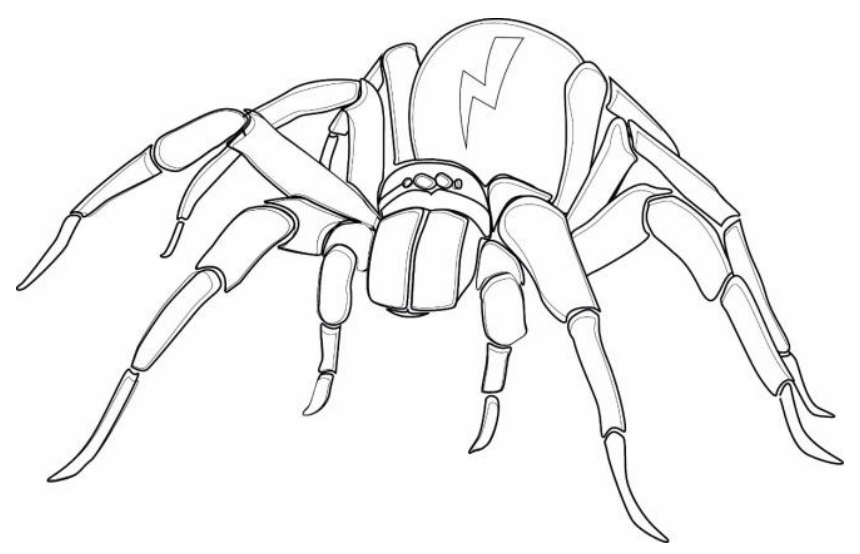




\title{
From lab to pilot scale: Melt-electrospun nanofibers of polypropylene with conductive additives
}

\begin{abstract}
In Chapter 3, the feasibility of fabricating polypropylene (PP) nanofibers was investigated using conductive additives such as sodium stearate ( $\mathrm{NaSt}$ ), sodium oleate $(\mathrm{NaOl})$ and the antistatic agent Irgastat during melt electrospinning with a single nozzle lab and a 600-nozzle pilot-scale device. Varying PP grades of high melt flow indices (MFI $=450$ $1200 \mathrm{~g} / 10 \mathrm{~min}$ ) were used with different amounts of additives. The effects of the additives on the fiber diameters, thermal properties, electrical conductivity and polymer degradation were investigated. On a lab scale, fiber diameters of less than $500 \mathrm{~nm}$ were achieved with the compound of PP HL712FB, 4\% (w/w) NaSt and 2\% (w/w) Irgastat. The lab scale device was extended by a heatable spinning chamber, which affects fiber diameter reduction. The fabrication of nanofibers was in principle attributed to the increase in electrical conductivity with the introduction of the additives. On a pilot scale, the smallest fiber diameter of $6.64 \mu \mathrm{m}$ could be achieved with PP HL508FB and $2 \%(\mathrm{w} / \mathrm{w})$ NaSt. The comparison between the production of the fibers with a single nozzle and the pilot scale device has revealed that a transfer of results is not possible without further ado. Due to the higher dwell time in the nozzle, a strong thermal degradation of the polymer could be detected with the high temperature size exclusion chromatography, whereby $\mathrm{NaOl}$ had the strongest influence on the thermal degradation. The high melt flow PP HL712FB and its compounds could not be processed with the pilot-scale device due to its low viscosity, resulting in an insufficient pressure built up within the spinneret. Another reason for the non-spinability of the material is the higher thermal and mechanical stress caused by the preceding melt preparation in an extrusion step. Further adjustments to the pilot-scale device are necessary to ensure a constant temperature distribution in the nozzle plate to achieve uniform fiber cross-sections. The implementation of an uneven collector has successfully led to an even deposition of the fibers to obtain an isotropic nonwoven fabric.
\end{abstract}

\section{Introduction}

Nano- and submicrofiber webs offer the advantageous property of an enormous specific surface area combined with high flexibility. Electrospinning is a widely-used process for the production of these nanofiber webs with a large surface-to-volume ratio and porosity with both micro- and nanopores [1]. Within the process, a polymeric fluid is stretched by electrostatic attraction under the presence of an external electric field. There are two different ways to produce nanofibers with this method, namely solution electrospinning and melt electrospinning [2]. Laboratory research and industrial production use these 
advantages of nanofibers in a wide variety of applications, such as medicine, energy and electronics, filtration and separation and textiles [1,3]. Melt electrospinning is a particularly interesting process for polymers that are poorly soluble and since no solvent is used, possible toxicity issues caused by the carry-over of the solvent into the final fiber product are also avoided. A further advantage of the melt electrospinning given by the absence of the solvent and thus a stable jet formation is the direct writeability, which is currently being increasingly investigated in the field of tissue engineering. The fiber diameters achieved are in the low micrometer to sub-micrometer range, whereby targeted three-dimensional structures can be reproduced [4-8]. Various thermoplastic polymers have been investigated for melt electrospinning, e.g. polyethylene, polypropylene, polycaprolactone, polyurethane, polylactic acid and poly (glycolide-colactide) [9-15]. Nevertheless, with melt electrospinning limiting factors such as difficulties resulting from the high temperature influence, high viscosity and low conductivity of polymer melts have to be taken into account compared with its counterpart process solution electrospinning $[14,16,17]$. In order to overcome polymer conductivity issues, a few studies on diverse additives - including sodium oleate, sodium chloride and sodium stearate - have been carried out, resulting in lower fiber diameters compared with an additive-free fiber production [18-20]. A key challenge for industry is the ability to upscale the nanofiber production within the melt-electrospinning process. Recent device developments such as multiple needle and needleless configurations have demonstrated a roadmap to overcome the low flow rate of melt electrospinning - typically in the $\mu \mathrm{g} / \mathrm{h}$ range - and therefore increase the fiber productivity [21]. Prototypes with umbellate nozzles containing 60 spinnerets can achieve maximum product deposition rates of $\sim 36 \mathrm{~g} / \mathrm{h}$ [22, 23]. In literature, the multi-needle configuration by Hacker et al. is currently the largest upscaled melt-electrospinning device with 64 nozzles installed [19].

The main objective of this study is to fabricate nanofibers based on PP and conductive additives with a novel, pilot-scale melt-electrospinning device. Challenges, solution approaches and achievements within the development of the 600-nozzle meltelectrospinning device are presented. A comparison with the production of nanofibers with a conventional, single jet laboratory melt-electrospinning device is drawn. The effects of viscosity and electrical conductivity as well as the influence of using different polymer grades and varying percentage $(\%(w / w))$ of the additives on the fiber diameter are investigated. In addition, thermal properties and polymer degradation issues are discussed.

\section{Experimental}

\section{Materials}

The isotactic PP melt blowing grades PP HL504FB, PP HL508FB and PP HL712FB with increasing MFI (450 g/10 min, $800 \mathrm{~g} / 10 \mathrm{~min}$ and $1200 \mathrm{~g} / 10 \mathrm{~min}$ at $230{ }^{\circ} \mathrm{C}$ and $2.16 \mathrm{~kg}$ ) 
from Borealis AG, Vienna, Austria were used for melt electrospinning [24]. The PP grades used have a narrow molecular weight distribution and a melting temperature of $158^{\circ} \mathrm{C}$. The conductive additives used here include sodium stearate from Alfa Aesar, Karlsruhe, Germany, sodium oleate from Carl Roth GmbH + Co. KG, Karlsruhe, Germany and the antistatic agent Irgastat P16 supplied by BASF, Ludwigshafen am Rhein, Germany in powder state. Irgastat is a polymeric system based on polyamide/polyether block amide. The materials were processed using a laboratory extruder Haake MiniLab II of Thermo Scientific Inc., Waltham, United States for preexaminations. Selected material combinations were subsequently processed using a two-screw extruder LSM $34 \mathrm{GL}$ from Leistritz AG, Nürnberg, Germany and granulated using a cutting mill C13.20sv from Wanner Technik GmbH, Wertheim-Reicholzheim, Germany. In addition to the additives used for melt electrospinning known from literature, the selection of additive concentrations and composition is based on the dissertation results of Hacker, who used the melt blowing polymers PP HL504FB, PP HL508FB and PP HL712FB for upscaling to the 64-nozzle system [25]. The results of the following five compounds will be discussed in further detail below:

- PP HL504FB / 4\% (w/w) NaSt / 2\% (w/w) Irgastat

- PP HL508FB / 2\% (w/w) NaSt

- PP HL712FB / 4\% (w/w) NaOI

- PP HL712FB / $2 \%(w / w)$ Irgastat

- PP HL712FB / 4\% (w/w) NaSt / 2\% (w/w) Irgastat

\section{Characterization of polymer compounds}

The data for polymer analysis in terms of rheological, thermal and molecular properties, as well as electrical conductivity were provided and interpreted by Jonas Daenicke of University Erlangen (Erlangen, Germany). The results are used to support the spinning process development and plant design.

Rheological characterization of the materials was performed with a focus on determining the zero shear viscosity. Frequency sweep measurements were performed on a Gemini Advanced Rheometer from Bohlin Instruments Ltd., Cirencester, United Kingdom. For the experiments, plate-to-plate geometry was used. The evaluation of the zero shear viscosity $\eta_{0}$ was carried out by fitting the experimental data with the Carreau-Yasuda equation [26]:

$$
\eta^{\prime}(\dot{\mathrm{Y}})=\frac{\eta_{0}}{\left(1+\left(\frac{\dot{\mathrm{Y}}}{\dot{\mathrm{Y}}_{\mathrm{c}}}\right)^{\mathrm{n}}\right)^{\mathrm{m}}}
$$

The equation is a generalization of the Newtonian model and it describes the variation of viscosity $\eta$ ' with shear rate $\dot{\gamma}$. The zero shear viscosities of the materials were determined at a measurement temperature of $210^{\circ} \mathrm{C}$. The dependency of the zero viscosity on the temperature was investigated as an example for PP HL712FB in a 
temperature range of $170-250^{\circ} \mathrm{C}$. Further rheological experiments were carried out to investigate the degradation behavior of the material. For this purpose, time sweeps measurements were made exemplary for PP HL508FB / 2\% (w/w) NaSt over a period of one hour at a constant shear rate and a temperature of $210^{\circ} \mathrm{C}$. The temperature was adapted to the temperature of the laboratory melt-electrospinning experiments.

In order to analyze the conductivity $\sigma$ of the polymer melts of the various compounds, electrical resistance measurements with a modified setup were made using a Gemini Advanced Rheometer of Bohlin Instruments Ltd., Cirencester, United Kingdom, performed with a $25 \mathrm{~mm}$ plate-to-plate geometry. For electrical conductivity measurements, the rheometer was equipped with a resistance measurement device 2400 Source Meter from Keithley Instruments, Cleveland, Ohio, United States, where the contact was made via the measuring geometry. The polymer conductivity was calculated via the determined electrical resistance. The measurements were carried out at a temperature of $210^{\circ} \mathrm{C}$.

Molecular analysis of the samples was performed with high temperature size exclusion chromatography (HT-SEC; PL-GPC 220, Polymer Laboratories Limited, United Kingdom) combined with a multi-angle laser light scattering (MALLS; Dawn EOS, Wyatt Technologies, United States). The number average molecular weight $M_{n}$, the weightaverage molecular weight $\mathrm{M}_{\mathrm{w}}$ and the polydispersity index (PDI) for the polypropylenes and the compounds used were determined. Additionally, investigations of melting temperatures and enthalpies were performed by differential scanning calorimetry (DSC). A Q2000 DSC from TA Instruments, Newcastle, United Kingdom was used for the measurements. Overall, two heat runs and one cooling run were carried out in a temperature range between -40 and $200{ }^{\circ} \mathrm{C}$ with a heating rate of $10 \mathrm{~K} / \mathrm{min}$. While for polyolefin the first heating run primarily serves to eliminate the thermal history, only the second one is relevant for the evaluation.

\section{Melt-electrospinning equipment}

In order to evaluate the general processability and fiber formation of the materials, a laboratory, self-made single-fiber melt-electrospinning system was used. Typically, the system comprised the five major components: a temperature controller, high-voltage power supply, heating elements, syringe pump and collector. EW 1133-4 temperature controllers from Eurotherm, Worthing, United Kingdom and NiCr-Ni thermocouples were used to control the melting and climatic chamber temperature. In order to apply a voltage between 30.0 and $50.0 \mathrm{kV}$, the HVG-P60-R-EU high-voltage generator from Linari Engineering, Pisa, Italy with a voltage range of 0.0 to $60.0 \mathrm{kV}$ was used. In this meltelectrospinning experiments, the voltage was applied to the collector with simultaneous grounding of the spinneret. The syringe pump type BSP-99M from Linari Engineering, Pisa, Italy is suitable for syringes between $5.0 \mathrm{ml}$ and $50.0 \mathrm{ml}$, whereby the delivery rate could be varied. Cannulas from Unimed SA, Lausanne, Switzerland with an inner diameter of $0.4 \mathrm{~mm}$ and a blunt end were used as nozzles. A flat aluminium plate 
$(\varnothing 7.0 \mathrm{~cm})$ served as a collector, which could be placed at different distances. A schematic diagram of the system is shown in Figure 3.1.

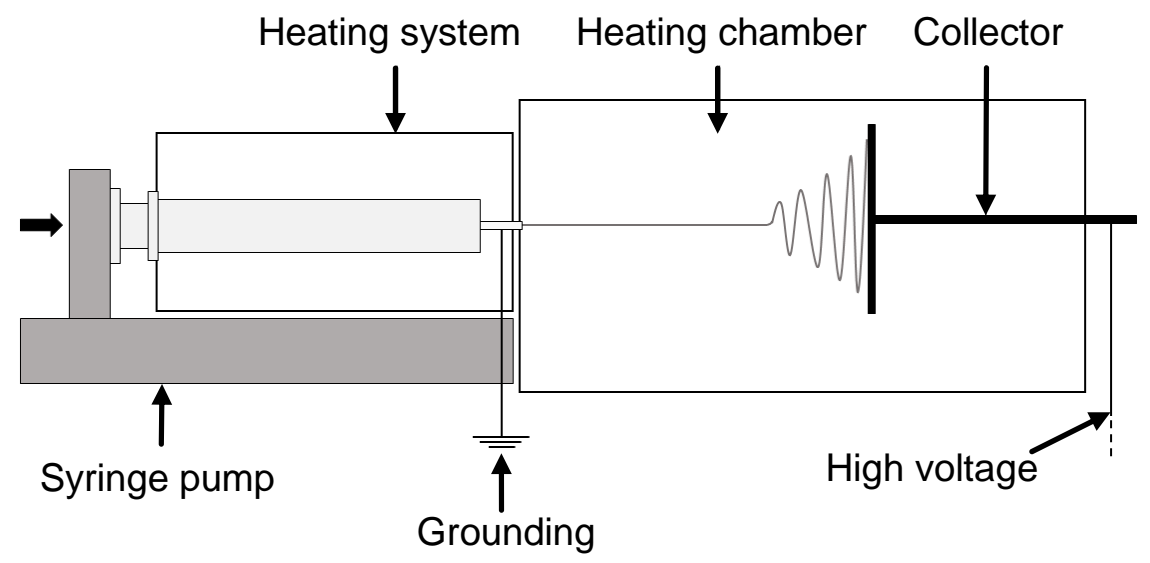

Fig. 3.1: Schematic diagram of the laboratory melt-electrospinning system.

The single-fiber melt-electrospinning trials were carried out at a melt outlet temperature of $210^{\circ} \mathrm{C}$, a collector distance of $10 \mathrm{~cm}$ and an applied voltage of $45 \mathrm{kV}$. Experiments were carried out with and without a climatic chamber, using a temperature of $100{ }^{\circ} \mathrm{C}$. The climate chamber provides an insulated spinning space and is tempered by hot air.

Upscaling the melt-electrospinning process beyond current state-of-the-art technologies was carried out with a newly-developed prototype including a spinneret with 600 nozzles. The nozzle plate of the developed melt-electrospinning device is shown in Figure 3.2.

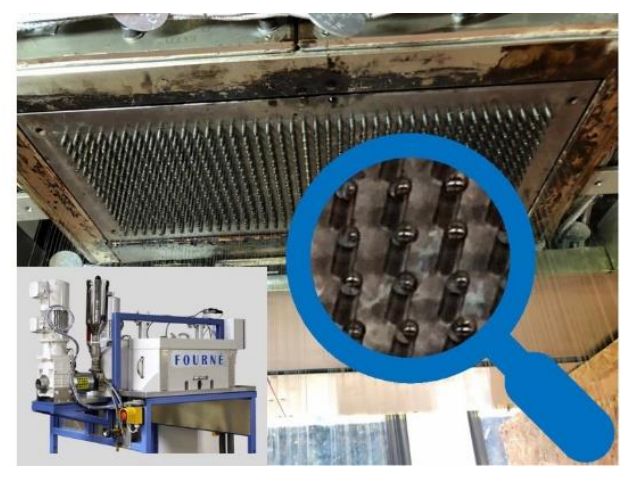

Fig. 3.2: 600 -nozzle plate of the melt-electrospinning prototype.

Each nozzle had a diameter of $0.3 \mathrm{~mm}$ and the nozzles were $8 \mathrm{~mm}$ apart of each other. A three-plate spinneret construction comprising a polymer inlet, distribution and nozzle plate was used. A fine-pored sinter plate was installed between the distributor and the nozzle plate. The plates were sealed against each other through a high specific contact load and additional aluminum seals at the polymer inlet. The constant supply of polymer melt was ensured by a speed adjustable single-screw extruder and spinning pump. Heating elements around the spinning unit were integrated. A collector with an uneven surface was used instead of a conventional plate collector. With the nozzle/collector pairing installed in the melt-electrospinning prototype, nonwovens over a width of 
$340 \mathrm{~mm}$ could be continuously produced. The distance between the collector and the nozzle plate was $11 \mathrm{~cm}$. A positive voltage of $60 \mathrm{kV}$ was applied to the collector using a high-voltage supply (Eltex KNH65). As with the laboratory system, the voltage was applied to the collector with simultaneous grounding of the spinneret.

The results obtained from the experiments with the conventional single-fiber meltelectrospinning system were transferred to the prototype device. The fiber formation, process stability and thus specifically-optimized process parameters such as the spinning temperature and throughput were investigated.

\section{Characterization of fibers}

The resulting fiber diameters were investigated via digital light microscopy (VHX-2000; Keyence Corporation, Osaka, Japan) using an VH-Z250R objective with a magnification between 250 and 2,500 and a Gemini scanning electron microscopy (SEM) (Carl Zeiss $A G$, Oberkochen, Germany) with an accelerating voltage of $3 \mathrm{kV}$. The samples were attached to the sample holders with an adhesive pad and sputtered with gold for 30 seconds prior to the SEM analysis using a Q150T S sputter coater (Quorum Technologies, Lewes, United Kingdom). The SEM images were evaluated using the Fiji ImageJ software. Molecular analysis of the fibers was again performed by HT-SEC. The number average molecular weight $\mathrm{Mn}$, the weight-average molecular weight $\mathrm{Mw}$ and the polydispersity index (PDI) of the fibers were determined to draw conclusions about the degradation behavior of the materials used during the spinning process.

\section{Results and discussion}

\section{Effects of viscosity}

The results of the frequency sweep measurements to determine the zero shear viscosities and standard deviations (SD) of the basic materials PP HL508FB and PP HL712FB as well as their fabricated compounds are summarized in Table 3.1. PP HL712FB has a significantly lower zero shear viscosity, which was expected due to the significantly higher MFI value. Compared with PP HL508FB, the zero shear viscosity of PP HL712FB is about one-third smaller. The results of the compounds reveal especially with sodium stearate and sodium oleate - an increase of the zero shear viscosity, whereas Irgastat P16 has only a minor influence on the viscosity. Increasing the zero shear viscosity of the material by using sodium stearate is contrary to expectations, given that the additive also acts as a lubricant between the polymer chains [27]. 
Tab. 3.1: Zero viscosities of the base materials PP HL508FB and PP HL712FB and their compounds.

\begin{tabular}{|l|l|l|}
\hline Material & Zero viscosity $\mathbf{n}_{\mathbf{0}}$ & $\mathbf{S D}\left(\mathbf{n}_{\mathbf{0}}\right)$ \\
\hline & $\mathbf{( P a \cdot s )}$ & $\mathbf{( P a \cdot s )}$ \\
\hline PP HL508FB & 15.18 & 0.27 \\
\hline PP HL712FB & 9.62 & 0.38 \\
\hline PP HL508FB/ 2\% (w/w) NaSt & 19.02 & 0.36 \\
\hline PP HL712FB/ 4\% (w/w) NaOI & 22.35 & 1.13 \\
\hline PP HL712FB/ 2\% (w/w) Irgastat & 10.35 & 0.26 \\
\hline
\end{tabular}

Since a higher viscosity usually leads to larger fiber diameters within the meltelectrospinning process, a reduction of the fiber diameter can still be achieved through increasing the electrical conductivity by the additives added [19]. The complex viscosity curves for PP HL712FB - considering the dependency on the temperature - are shown in Figure 3.3.

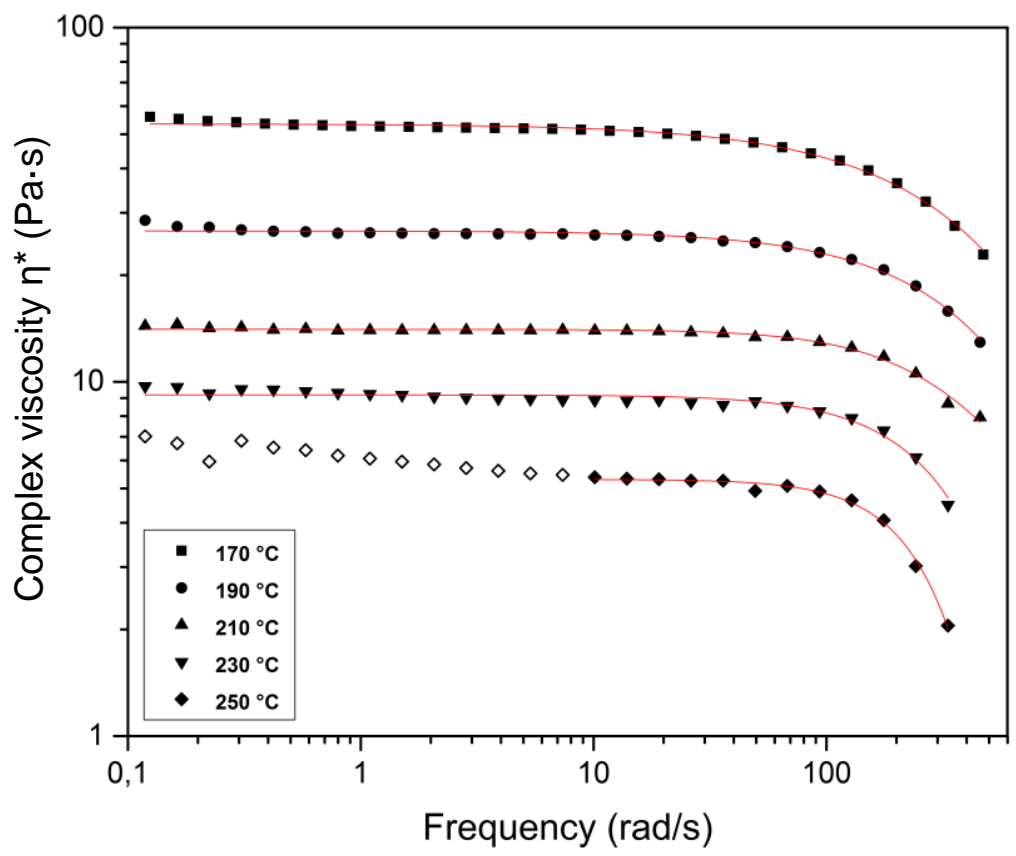

Fig. 3.3: Dependence of the zero viscosity on the temperature for PP HL712FB.

A significant reduction in viscosity can be seen with increasing temperature. The value of the zero shear viscosity at $250^{\circ} \mathrm{C}$ can only be considered as an approximation given that the determination only relates to the high frequency range of the measurement. Since the initial frequency range shows an excessive decrease in viscosity, no clear Newtonian plateau is formed. The reason for this could be the variation in viscosity in the range of low frequencies due to the onset of degradation of the material due to both 
thermal and shear stress, while the resulting low viscosity values could be outside the possible measuring range. Using the example of PP HL712FB and PP HL508FB/ $2 \%(w / w) ~ N a S t$, the additional, rheological time sweep measurement carried out to investigate the degradation of the material shows a steady decrease in viscosity over time at a constant shear rate due to thermal and mechanical material stress (Figure 3.4).

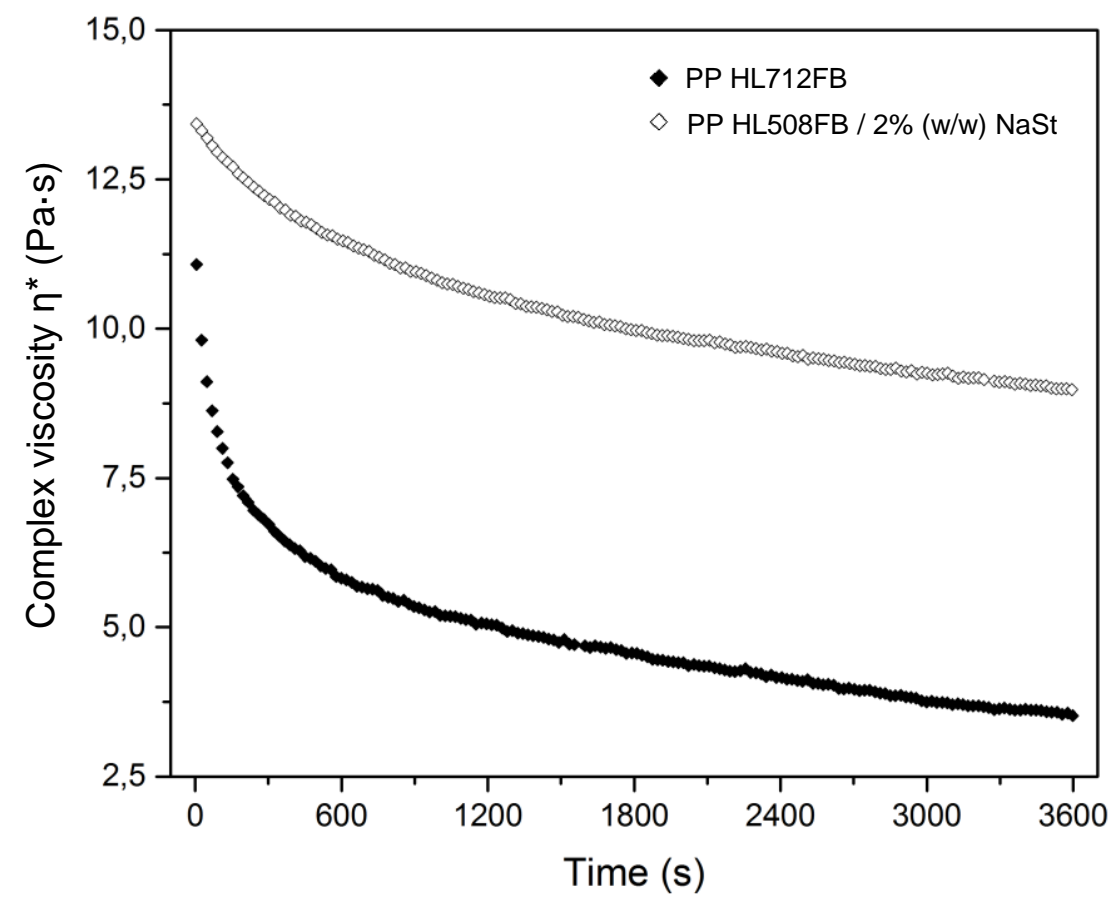

Fig. 3.4: Time sweep measurement of the compound PP HL508FB with $2 \%(\mathrm{w} / \mathrm{w}) \mathrm{NaSt}$ at $210^{\circ} \mathrm{C}$.

As expected, the PP HL712FB is characterized by a lower viscosity curve. In addition, a stronger decrease in viscosity is apparent at the beginning, which may indicate a stronger degradation of the material. Since the zero shear viscosity is directly related to the weight-average molecular weight, the decrease can be justified by a degradation of the material. Therefore, it seems necessary to optimize the compound used, including regarding the degradation and supplementary to ensure a short residence time of the material within the development of the melt-electrospinning prototype device. This material behavior can be observed with all selected additives and base materials and is subsequently assigned using the GPC data.

\section{Effects of electrical conductivity}

The conductivity measurements show the expected increase in conductivity with the addition of an additive. The measurements results are summarized in Table 3.2. 
Tab. 3.2: Results of the electrical conductivity measurements.

\begin{tabular}{|l|l|l|}
\hline Material & $\begin{array}{l}\text { Conductivity } \boldsymbol{\sigma} \\
(\mathbf{S} / \mathbf{c m})\end{array}$ & $\begin{array}{l}\text { SD }(\boldsymbol{\sigma}) \\
(\mathbf{S} / \mathbf{c m})\end{array}$ \\
\hline PP HL508FB & $3.07 \mathrm{E}-10$ & $1.25 \mathrm{E}-10$ \\
\hline PP HL712FB & $1.82 \mathrm{E}-10$ & $3.95 \mathrm{E}-11$ \\
\hline PP HL508FB/ 2\% (w/w) NaSt & $2.12 \mathrm{E}-09$ & $1.10 \mathrm{E}-10$ \\
\hline PP HL712FB/ 4\% (w/w) NaOI & $1.18 \mathrm{E}-08$ & $2.26 \mathrm{E}-09$ \\
\hline PP HL712FB/ 2\% (w/w) Irgastat & $4.44 \mathrm{E}-10$ & $1.06 \mathrm{E}-10$ \\
\hline PP HL712FB/ 4\% (w/w) NaSt / 2\% (w/w) Irgastat & $8.73 \mathrm{E}-09$ & $1.78 \mathrm{E}-09$ \\
\hline
\end{tabular}

PP as a well-known good electrical insulator showing an electrical conductivity in the range of $10^{-10} \mathrm{~S} / \mathrm{cm}$ at a temperature of $210^{\circ} \mathrm{C}$. The additives $\mathrm{NaSt}$ and $\mathrm{NaOl}$ lead to a significant increase depending on the concentration, whereas the antistatic Irgastat P16 shows only a small influence on the material parameter. For this purpose, further investigations were carried out depending on the additive concentration, although these are not considered in detail here. Figure 3.5 provides an overview of the electrical conductivity of the analyzed materials.

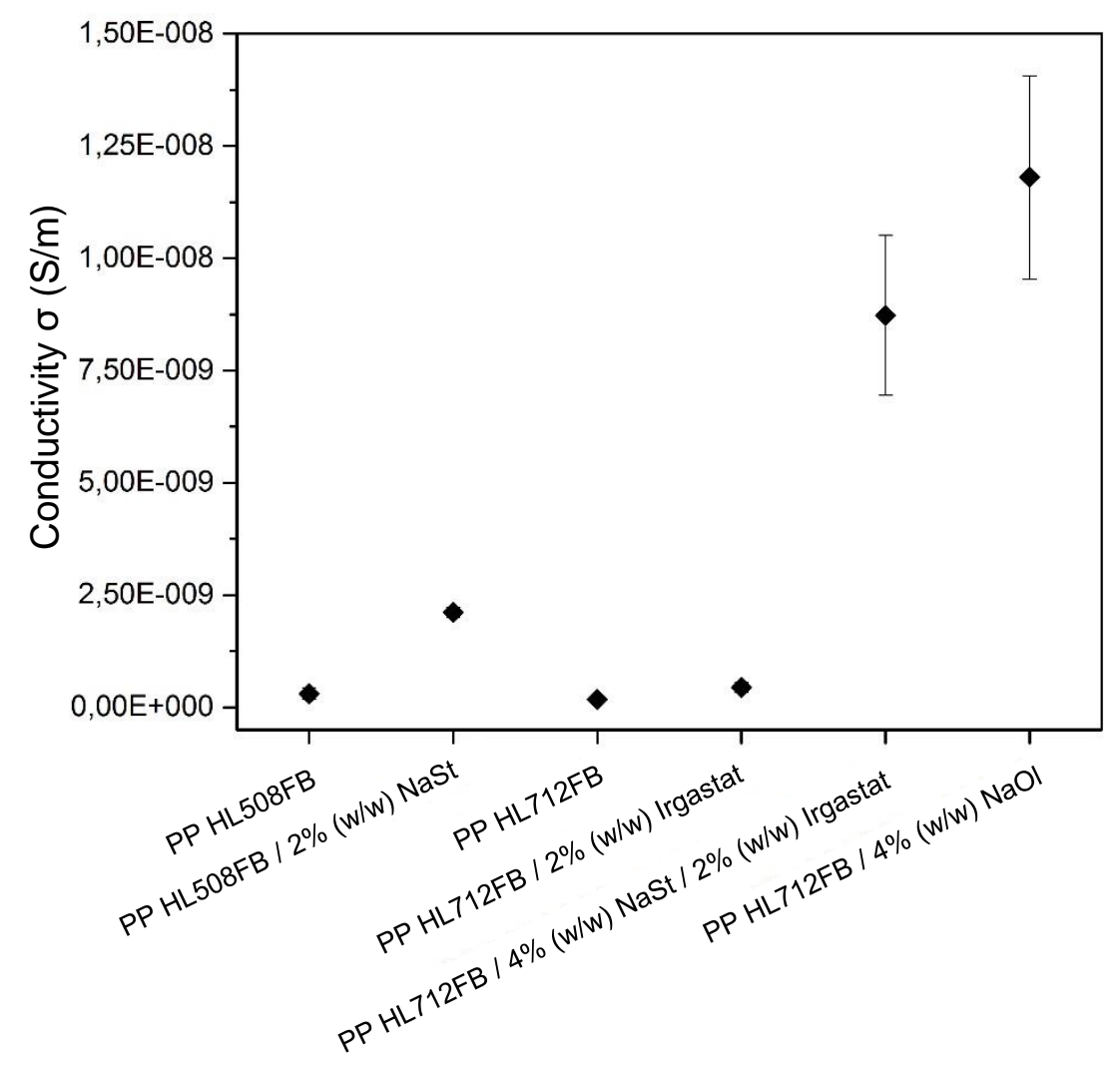

Fig. 3.5: Electrical conductivity in relation to the compounds and their additive concentrations. 
The electrical conductivity in the polymer material is determined by the generation and mobility of the charge carriers or ions. As previously described by Lin et al., the increase in the electrical conductivity can be ascribed to the effect of ions from the dissociation of the additives. The charge-carrying capacity is thus increased during melt electrospinning, which causes increased whipping instabilities through an increased charge repulsion in the polymer jet. Consequently, the jet is exposed to stronger stretching forces, resulting in finer fiber diameters [28].

\section{Thermal properties}

Determination of the melting temperatures and enthalpies was carried out by DSC. In Figure 3.6, the curves of the materials PP HL508FB, PP HL712FB and PP HL508FB/ $2 \%(w / w)$ NaSt are exemplary shown.

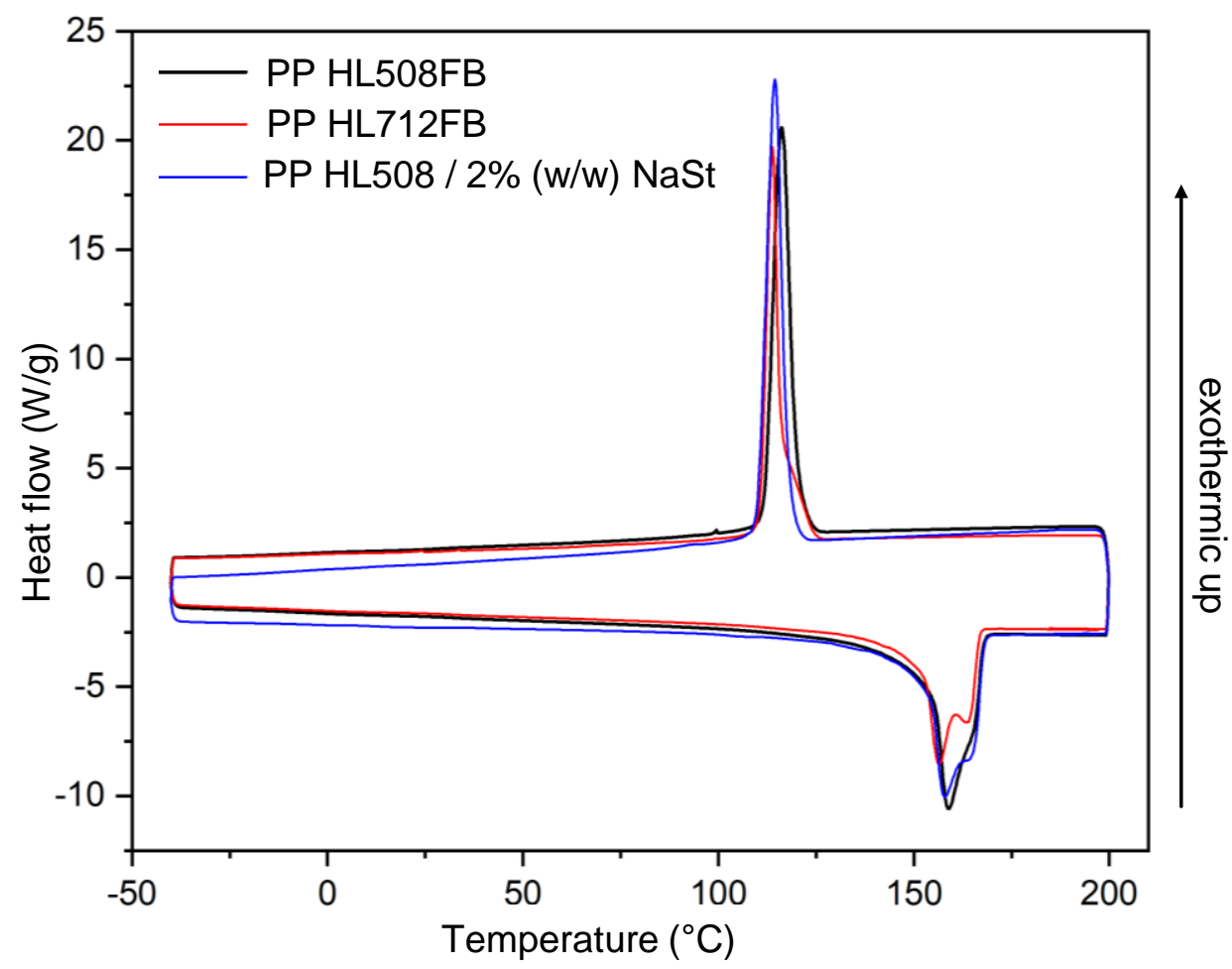

Fig. 3.6: DSC curve of material PP HL712FB $-2^{\text {nd }}$ heating run and $1^{\text {st }}$ cooling run.

The curves present the second heating run and the first cooling run of the DSC measurement. The double peak in the melting range of the PP HL712FB appears due to a beta-phase formation [29]. The melt blowing polypropylene grades used with high MFI values already contain appropriate beta-phase nucleating agents. Depending on the application, beta-phase formation is desired in nonwoven fabric and fiber production due to the influence on the material properties. This effect is not that strong for PP HL508FB, which can be seen in Figure 3.6. The corresponding values for the base materials PP HL508FB and PP HL712FB and their compounds used are listed in Table 3.3. The addition of additives to the base materials causes no apparent change in the melting point, although an increase in melt enthalpies can be observed. 
Tab. 3.3: Melting temperatures and enthalpies of the base materials and their compounds.

\begin{tabular}{|l|l|l|}
\hline Material & $\begin{array}{l}\text { Melting Point } \mathbf{T}_{\mathbf{m}} \\
\left({ }^{\circ} \mathbf{C}\right)\end{array}$ & $\begin{array}{l}\text { Melting enthalpy } \\
(\mathbf{J} / \mathbf{g})\end{array}$ \\
\hline PP HL508FB & 158.9 & 78.7 \\
\hline PP HL712FB & 156.5 & 73.9 \\
\hline PP HL508FB/2\% (w/w) NaSt & 158.0 & 93.8 \\
\hline PP HL712FB/ 4\% (w/w) NaOI & 156.6 & 91.0 \\
\hline PP HL712FB/ 2\%(w/w) Irgastat & 156.4 & 97.3 \\
\hline
\end{tabular}

\section{Molecular weight distribution}

The evaluation of the GPC analysis shows that the values for the number average and weight-average molecular weight are in a small order of magnitude range, which is justified due to the choice of polypropylene with a low viscosity and high MFI value optimized for the melt blowing process. The compounds produced have slightly lower values due to thermal and shear stresses during the manufacturing process. A further decrease of the molecular weight can be observed for the manufactured fiber products in laboratory scale as well as with the pilot-scale melt-electrospinning device. With the prototype plant, a molecular weight loss of about $25 \%$ can be determined due to an average dwell time of $50 \mathrm{~min}$. Furthermore, the GPC evaluation reveals that the material degrades more in the presence of $\mathrm{NaOl}$ compared with the other additives, even though different additive concentrations were used.

The molecular weights of the base materials, compounds and the resulting fiber products are summarized in Table 3.4. 


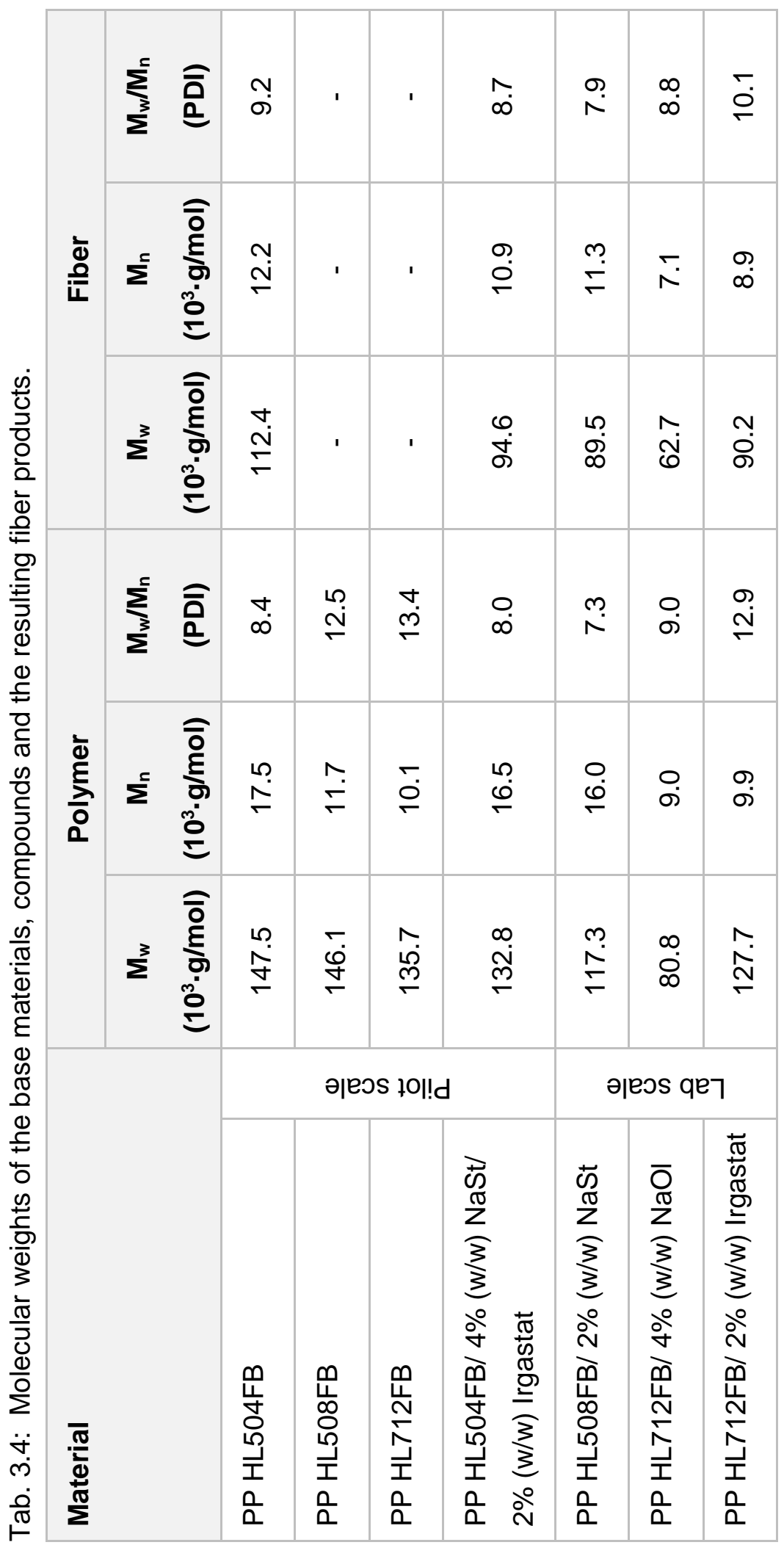




\section{Fiber diameters and distribution}

The processability of the materials was investigated with the single-fiber meltelectrospinning device. The modified materials are characterized by a constant fiber formation. Fiber diameters in the low $\mu \mathrm{m}$ range could be achieved. In addition, the typical formation of a Taylor cone as well as a typical fiber deposition for electrospinning can be

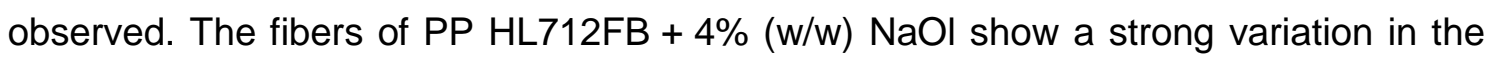
fiber diameter (a), while the additive Irgastat leads to a more uniform diameter distribution (b) (Figure 3.7).
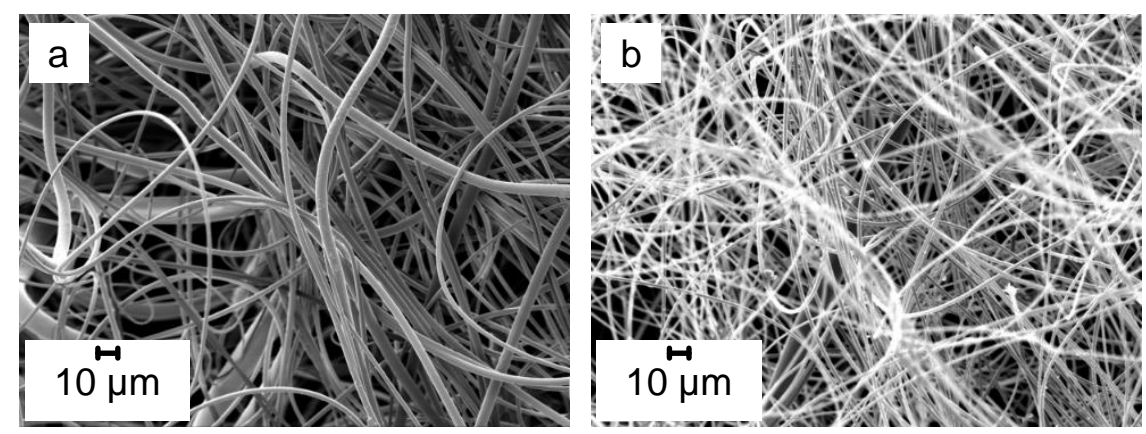

Fig. 3.7: SEM images of the fibers from the laboratory spinning processes; PP HL712FB with 4\% (w/w) NaOl (a) and PP HL712FB with $2 \%(w / w)$ Irgastat (b).

This is due to the previously-described increased material degradation caused by the influence of the additive $\mathrm{NaOl}$. On a laboratory scale, the finest fiber diameter of less than $500 \mathrm{~nm}$ was achieved with the material PP HL712FB + 4\% (w/w) NaSt and $2 \%(w / w)$ Irgastat in the presence of the climatic chamber. However, with the material PP HL508FB, a fiber diameter of $2.07 \mu \mathrm{m}$ was also achieved on average.

Different challenges such as a uniform melt flow in the spinneret and an even distribution of the spun fibers on the collector must be considered when upscaling a meltelectrospinning device from a smaller to a higher number of nozzles. The transfer of the results from laboratory to pilot scale is not easily applied, mainly due to the longer dwell time in the nozzle compared with the syringe and the lower shear rates. For this reason, it was - for example - not possible to gain a homogeneous process with the compound PP HL712FB / 4\% (w/w) NaOl. Due to the stronger degradation of the material, drops of the low-molecular decomposition products had to be removed and the nozzle plate had to be cleaned or rinsed after each series of tests. Furthermore, the low viscosity of the material and the low shear rates prevent the necessary pressure build-up for a uniform fiber formation over the entire nozzle cross-section, despite the targeted three-plate nozzle design and the additional fine-pored sinter plate. Without a corresponding pressure in the nozzle, the polymer is not pressed out of the nozzle during spinning but only attracted by the electric field. Consequently, no sufficient polymer flow can be ensured and the filaments tear off. On a laboratory scale, PP HL712FB with Irgastat and $\mathrm{NaSt}$ reveals the best results and fiber finesses of less than $500 \mathrm{~nm}$. The same material 
does not lead to filament formation on the pilot-scale system, but only to droplet formation, which can be explained by the lower viscosity of the material itself on the one hand and on the other hand by a higher thermal and mechanical stress on the material in the pilot-scale spinning device due to the preceding extrusion step. This process step is responsible for the melt preparation and leads to material degradation due to the applied stress. These factors lead to a more difficult pressure build-up in the nozzle plate. Due to the differences in melt preparation between the lab and the pilot-scale system, the thermal prehistory of the material cannot be compared. Due to this problem, the higher viscous material PP HL504FB and PP HL508FB were used on a pilot scale. Additionally, the polymer throughputs were increased, resulting in a low pressure buildup within the spinneret and a stabilized fiber formation. However, at the same time, the filament diameters obtained are not in the sub-micrometer or nanometer range. Without additives, no fiber formation on the pilot scale was obtained at all. The material-adapted spinning parameters in laboratory/pilot scale and resulting fiber diameter are summarized in Table 3.5 . 


\begin{tabular}{|c|c|c|c|c|c|c|c|c|}
\hline 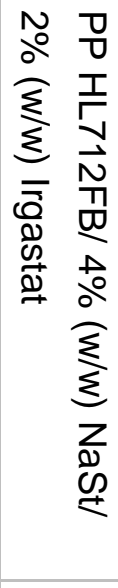 & 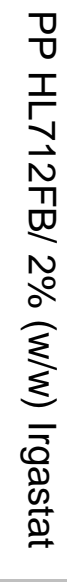 & 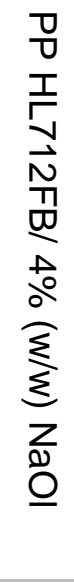 & $\begin{array}{l}0 \\
0 \\
I \\
5 \\
0 \\
0 \\
0 \\
00 \\
0 \\
N \\
0 \\
\vdots \\
\vdots \\
\vdots \\
\vdots \\
0 \\
0\end{array}$ & 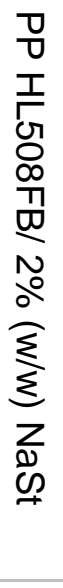 & 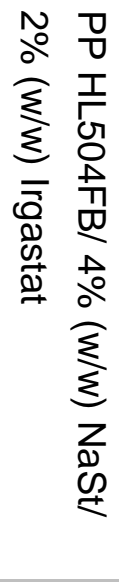 & & & $\begin{array}{l}3 \\
\stackrel{3}{D} \\
\frac{D}{10} \\
\cong\end{array}$ \\
\hline \multicolumn{4}{|c|}{ Lab scale } & \multicolumn{2}{|c|}{ Pilot scale } & & & \\
\hline ' & ' & ' & 1 & 공 & 옴 & @ి & 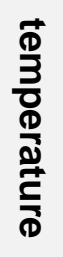 & 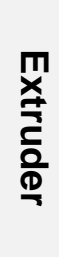 \\
\hline$\frac{N}{O}$ & $\frac{N}{O}$ & $\frac{N}{O}$ & $\stackrel{N}{O}$ & $\frac{N}{O}$ & N & $\widehat{\varrho}$ & 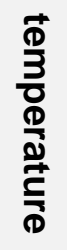 & $\begin{array}{l}Z \\
\mathbf{O} \\
\mathbf{N} \\
\frac{1}{D}\end{array}$ \\
\hline ○े & $\stackrel{\circ}{\circ}$ & $\stackrel{\circ}{\circ}$ & $\stackrel{\circ}{\circ}$ & $\stackrel{\circ}{\dot{\omega}}$ & $\stackrel{\circ}{\dot{\omega}}$ & $\widehat{3}$ & 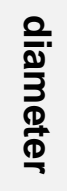 & $\begin{array}{l}Z \\
\mathbf{N} \\
\mathbf{N} \\
\frac{1}{0}\end{array}$ \\
\hline 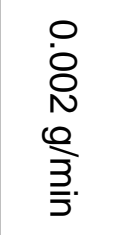 & 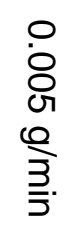 & $\begin{array}{l}\circ \\
\text { 옹 } \\
\text { ㅇ } \\
\stackrel{0}{3} \\
3\end{array}$ & $\begin{array}{l}0 \\
\dot{8} \\
0 \\
0 \\
0 \\
3 \\
3\end{array}$ & $\begin{array}{l}\vec{\sigma} \\
\overrightarrow{0} \\
\frac{0}{3}\end{array}$ & $\begin{array}{l}\vec{\infty} \\
\overrightarrow{0} \\
3\end{array}$ & & & 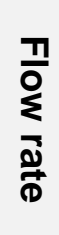 \\
\hline $\begin{array}{l}v \\
\text { 웅 } \\
\text { 잉 }\end{array}$ & ci & $\begin{array}{l}\omega \\
\phi \\
\phi\end{array}$ & بֶ & $\begin{array}{l}\infty \\
\infty \\
\infty \\
\infty\end{array}$ & $\begin{array}{l}\infty \\
\stackrel{ \pm}{*}\end{array}$ & $\widehat{\widehat{\jmath}}$ & 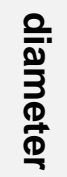 & $\begin{array}{l}\frac{\pi}{6} \\
\frac{\Phi}{1}\end{array}$ \\
\hline ' & $\overrightarrow{⿱ 亠 凶}$ & o̊ & $\stackrel{0}{\dot{\omega}}$ & $\stackrel{\vec{N}}{\stackrel{N}{N}}$ & $\begin{array}{l}\omega \\
\vec{a}\end{array}$ & ్ㅗㄹ & 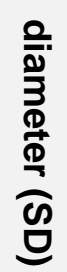 & $\begin{array}{l}\frac{\pi}{\sigma} \\
\frac{\mathbb{D}}{7}\end{array}$ \\
\hline
\end{tabular}


For PP HL508FB, a fiber diameter of $6.48 \mu \mathrm{m}$ was obtained on a pilot scale after process parameter optimization. In order to reduce the filament diameter, it is necessary to stretch the material jets in the area to the collector more efficiently. This can only be realized by a stronger interaction with the electric field or a climatic chamber around the spinning unit as used on a laboratory scale to avoid a rapid solidification of the polymer jets. In addition, the climatic chamber would favor a homogenization of the temperature distribution on the nozzle plate, whereby the variation of the fiber diameter could be reduced. The temperature distribution on the nozzle plate measured with an infrared thermometer at a set temperature of $230^{\circ} \mathrm{C}$ is shown in Figure 3.8. Fifteen different measuring points distributed symmetrically over the nozzle plate were measured. With a heating time of 4 hours at $230^{\circ} \mathrm{C}$, a temperature drop of up to $40^{\circ} \mathrm{C}$ can be measured from the polymer inlet to the front edge of the nozzle plate.

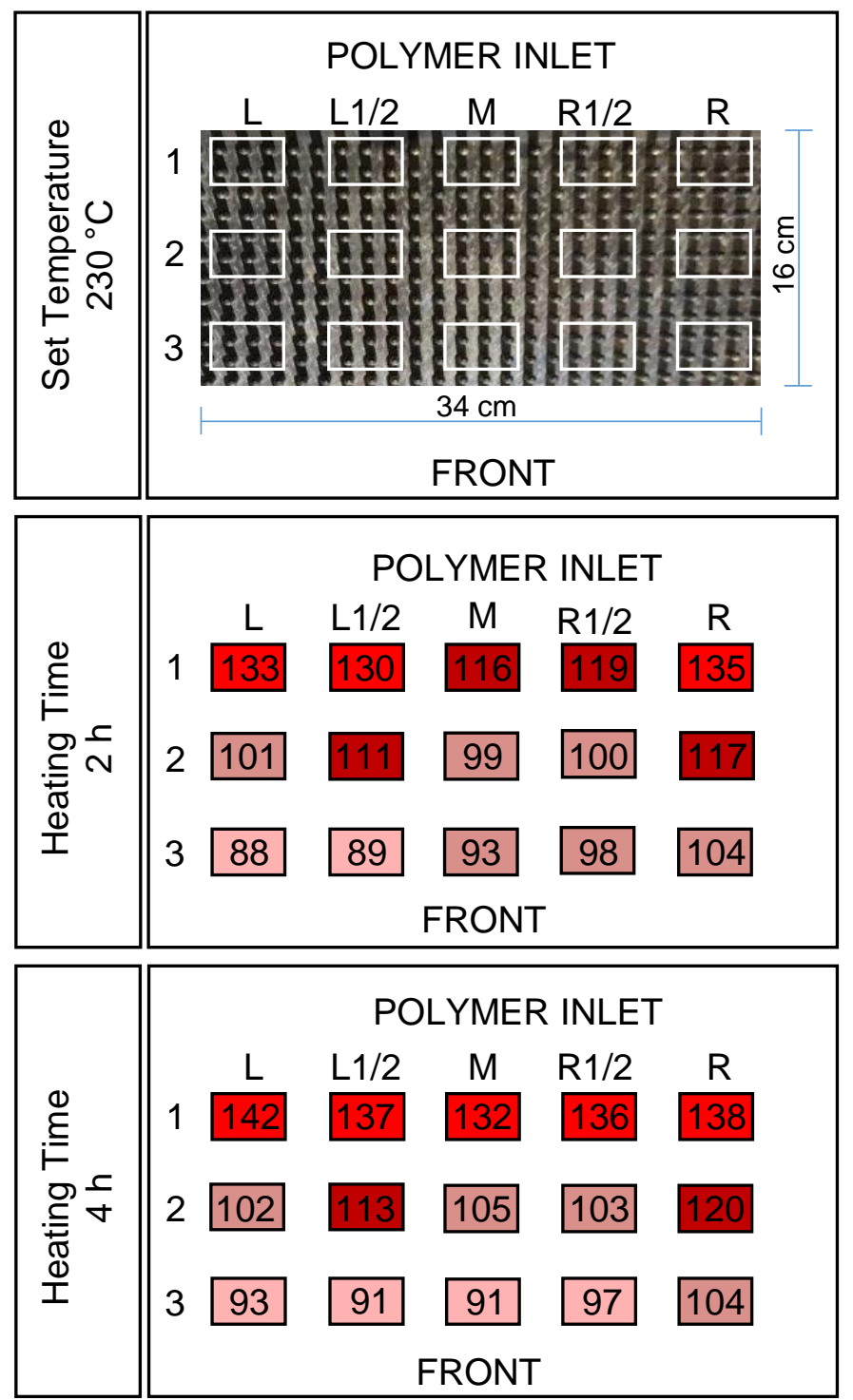

Fig. 3.8: Temperature distribution on 600-nozzle plate, measured after $2 \mathrm{~h}$ and $4 \mathrm{~h}$ at set temperature of $230^{\circ} \mathrm{C}$. 
Due to the uneven collector, a more even distribution of the fibers on the collector could be achieved compared to a plate collector. An optimal arrangement of the pins could not be determined, although tendencies can be recognized. A collector pin can be placed every $8 \mathrm{~mm}$. A narrow and offset pin order (a) with a diagonal distance of $2.6 \mathrm{~cm}$ ensures an even fiber deposit. An arrangement in a row with a horizontal distance of the collector pins of $2.4 \mathrm{~cm}$ and a vertical distance of $1.6 \mathrm{~cm}$ created a linear distribution (b) (Figure 3.9).
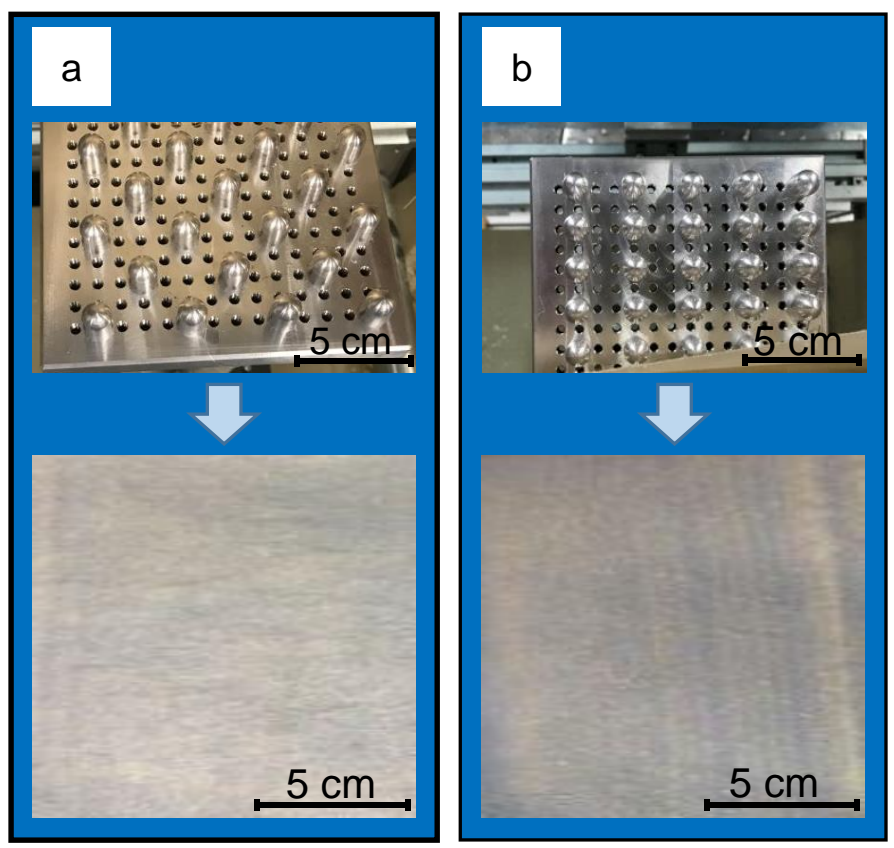

Fig. 3.9: Fiber distribution on collector plate with (a) narrow and offset pin order and (b) arrangement in a row.

\section{Conclusion and further perspective}

With the new pilot-scale melt-electrospinning device, the first polypropylene-based fibers in the low micrometer range were successfully produced. The experiments have shown that a transfer of the results from the lab to the pilot-scale melt-electrospinning device is not possible without further ado. The major issues are the dwell time in the spinneret compared to the syringe and the different shear rates. The high melt flow PP HL712FB could not be processed with the pilot-scale device due to its low viscosity, resulting in an insufficient pressure built up within the spinneret, which led to droplet formation instead of a continuous fiber formation. The additives - in particular sodium oleate - led to a strong degradation of the polymer, which required extensive cleaning of the devices from degradation products and impaired the formation of fibers. The integration of the climate chamber led to the finest fibers on a laboratory scale with a fiber diameter of less than $500 \mathrm{~nm}$ using the material combination PP HL712FB $+4 \%(\mathrm{w} / \mathrm{w}) \mathrm{NaSt}$ and $2 \%(w / w)$ Irgastat. On a pilot scale, the smallest fiber diameter of $6.64 \mu \mathrm{m}$ could be achieved with PP HL508FB $+2 \%(\mathrm{w} / \mathrm{w}) \mathrm{NaSt}$, which yields a fiber diameter of $2.07 \mu \mathrm{m}$ on a laboratory scale. In order to reduce the fiber diameter on a pilot scale, further plant- 
specific adjustments are required. This includes the integration of a climate chamber, which has already been successfully implemented on a laboratory scale and has made a decisive contribution to fiber formation in the nanometer range. Regarding the thermal degradation problem, the spinneret has to be optimized to reduce dwell times as well as maintaining a constant pressure loss and sufficiently low polymer throughputs. Through targeted regulation of the applied electrical voltage at the individual collector tips, the process could also be made accessible to other applications where a definite rather than a random fiber deposit is necessary, e.g. tissue engineering.

\section{Acknowledgements}

The authors acknowledge the support by the Federal Ministry of Economic and Energy as part of the Zentrales Innovationsprogramm Mittelstand (ZIM) for the project "ZIM EFeld". We also acknowledge the support of Fourné Maschinenbau GmbH (AlfterImpekoven, Germany), Pötter-Klima Gesellschaft für Nanoheiztechnik mbH (Georgsmarienhütte, Germany) and Schnick Systemtechnik GmbH \& Co. KG (Heiligenhaus, Germany), as well as the previous work on the development of the meltelectrospinning device at the Institute of Textile Technology (ITA), RWTH Aachen University (Aachen, Germany), carried out by Ms. Lützow and Mr. Hacker, former employees of the institute. 


\section{References}

[1] Bhat GS. Advances in Polymeric Nanofiber Manufacturing Technologies. Nanomater Mol Nanotechnol 2016; 5: 2324.

[2] Bhardwaj N., Kundu SC. Electrospinning: A fascinating fiber fabrication technique. Biotechnology Advances 2010; 28: 325-347.

[3] Liu Y, Deng R, Hao M, Yan H, Yang W. Orthogonal design study on factors effecting on fibers diameter of melt electrospinning. Polym Eng Sci 2010; 50(10):2074.

[4] Willerth SM. Electrospun Materials for Tissue Engineering and Biomedical Applications: Research, Design and Commercialization. Woodhead Publishing 2017.

[5] Wunner FM, Wille ML, Nanoon TG, Bas O, Dalton PD et al. Melt Electrospinning Writing of Highly Ordered Large Volume Scaffold Architectures. Adv. Mater. 2018; 30:1706570.

[6] Wunner FM, Maartens J, Bas O, Gottschalk K, De-Juan-Pardo E et al. Electrospinning writing with molten poly ( $\varepsilon$-caprolactone) from different directions - Examining the effects of gravity. Material Letters 2018; 216: 114-118.

[7] Eichholz KF, Hoey DA. Mediating human stem cell behaviour via defined fibrous architectures by melt electrospinning writing. Acta Biomaterialia 2018; 75: 140-151.

[8] Dayan CB, Afghah F, Okan BS, Yıldız M, Menceloglu Y et al. Modeling 3D melt electrospinning writing by response surface methodology. Materials \& Design 2018; 148: 87-95.

[9] Zhao F, Liu Y, Yuan H, et al. Orthogonal design study on factors affecting the degradation of polylactic acid fibers of melt electrospinning. J Appl Polym Sci 2012; 125: 2652-2658.

[10] Li X, Liu H, Liu J, et al. Preparation and experimental parameters analysis of laser melt electrospun poly(L-lactide) fibers via orthogonal design. Polym Eng Sci 2012; 52: 1964-1967.

[11] Larrondo L, St. John Manley R. Electrostatic fiber spinning from polymer melts. I. Experimental observations on fiber formation and properties. J Polym Sci Polym Phys 1981;19: 909-920.

[12] Dalton PD, Lleixà Calvet J, Mourran A, et al. Melt electrospinning of poly-(ethylene glycol-block- $\varepsilon$-caprolactone). Biotechnol J 2006; 1: 998-1006.

[13] Yoon Y, Park KE, Lee SJ, et al. Fabrication of microfibrous and nano-/microfibrous scaffolds: melt and hybrid electrospinning and surface modification of poly(L-lactic acid) with plasticizer. Biomed Res Int 2013: 309048. 
[14] Zhou H, Green TB, Joo YL. The thermal effects on electrospinning of polylactic acid melts. Polymer (Guildf) 2006; 47: 7497-7505.

[15] Brown TD, Dalton PD, Hutmacher DW. Direct Writing By Way of Melt Electrospinning. Adv. Mater. 2011; 23, 5651-5657.

[16] Nayak R. Polypropylene Nanofibers: Melt Electrospinning Versus Meltblowing. Engineering Material. Springer International Publishing 2017.

[17] Bubakir M, Barhoum A, Li H, Yang W. Handbook of Nanofibers. (1st edtn), Springer Nature 2017.

[18] Nayak R, Kyratzis IL, Truong YB, Padhye R, Arnold L. Melt-electrospinning of polypropylene with conductive additives. JMater Sci 2009; 47:6387-96.

[19] Hacker CJP, Seide G, Gries T, Thomas H, Moeller M. Electrospinning of polymer melt: steps toward an upscaled multi-jet process. In:Proc Int Confer Latest Advances in High Tech Textiles and Textile-Based Materials 2009: 71-6.

[20] Nayak R, Padhye R, Kyratzis IL, Truong YB, Arnold L. Effect of viscosity and electrical conductivity on the morphology and fiber diameter in melt electrospinning of polypropylene. Textile Research Journal 2013; 83: 606-617.

[21] Brown TD, Dalton PD, Hutmacher DW. Melt electrospinning today: An opportune time for an emerging polymer process. Progress in Polymer Science 2016; 56: 116-166.

[22] $\mathrm{Li} \mathrm{H}$, Chen $\mathrm{H}$, Zhong $\mathrm{X}$, et al. Interjet distance in needleless melt differential electrospinning with umbellate nozzles. J Appl Polym Sci 2014; 131: 40515.

[23] Liu Y, Zhao F, Zhang C, Zhang J, Yang W. Solvent-free preparation of poly(lactic acid) fibers by melt electrospinning using an umbrella-like spray head and alleviation of the problematic thermal degradation. J. Serb. Chem. Soc. 2012; 77:1071-1082.

[24] Borealis AG. Solutions for Fibres-Summary Data Sheet. https://www.borealisgroup.com/storage/Polyolefins/ConsumerProducts/Fibres/solutions-for-fibres.pdf (accessed 21 September 2018).

[25] Hacker C. Anlagenentwicklung für das Elektroschmelzspinnen von Feinstfaservliesstoffen für die Abwasseraufbereitung. Shaker Verlag 2014. RWTH Aachen University.

[26] Andrade LC, Petronílio J, Maneschy CE, Cruz DO. The Carreau-Yasuda Fluids: a Skin Friction Equation for Turbulent Flow in Pipes and Kolmogorov Dissipative Scales. J. of the Braz. Soc. of Mech. Sci. \& Eng. 2007; XXIX (No. 2): 162-167.

[27] Wypych G. PVC ADDITIVES. 2015; 45-88. 10.1016/B978-1-895198-84-3.500053. 
[28] Lin $\mathrm{T}$, Wang $\mathrm{H}$, Wang $\mathrm{X}$. The charge effect of cationic surfactants on the elimination of fibre beads in the electrospinning of polystyrene. Nanotechnology 2004; $15: 1375$.

[29] Varga J, Ehrenstein GW. Beta-modification of isotactic polypropylene. In: KargerKocsis J, editor. Polypropylene: An A-Z reference. Dordrecht: Springer Netherlands; 1999. p. 51-9. 


\section{Chapter 4}

Polylactic acid in melt electrospinning

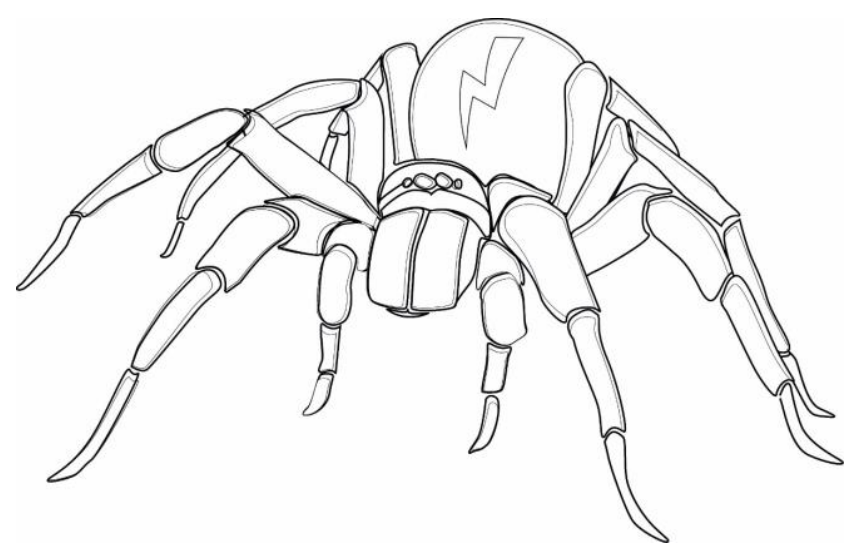




\section{Polylactic acid in melt electrospinning}

The biobased polymer polylactic acid (PLA) is introduced below and the advantages of this polymer versus polypropylene in the melt-electrospinning process are explained.

Polylactic acid is an environmentally friendly, plant-derived thermoplastic and thus can be used in conventional fiber extrusion processes. At least $85 \%(w / w)$ lactic acid ester units derived from naturally occurring sugars (sugar beets and corn) are contained in a lactic acid polymer. Although compostable and biodegradable according to EN 13432, PLA does not readily degrade unless it is exposed to high humidity and elevated temperatures $\left(\geq 60^{\circ} \mathrm{C}\right)$ which are usually just found in industrial composting plants [1]. The tensile strength of PLA fibers is comparable to those of polyethylene terephthalate fibers [2]. However, PLA has a low glass transition temperature of only $55-60{ }^{\circ} \mathrm{C}$ and thus, is less heat resistant than polyethylene terephthalate. In addition, the associated degradation of the polymer starts at temperatures $10^{\circ} \mathrm{C}$ above its melting temperature, which is between $130-175^{\circ} \mathrm{C}[2,3]$. It is also more flammable and less abrasion resistant. During processing, the high affinity to moisture and the possibility of hydrolysis when the polymer is exposed to ambient air must be taken into account [4].

NatureWorks (Minnetonka, United States) is the market leader in polylactic acid technology. Other manufacturers are Total Corbion PLA (Gorinchem, The Netherlands), Synbra (Etten-Leur, The Netherlands) and Hisun Biomaterials (Zhejiang, China) [5]. Worldwide, more than 400 million tons of plastic are produced annually, and in 2016, the quantity of PLA produced reached 217 thousand tons. By 2021 the production of PLA is expected to increase to 607 thousand tons [6]. Commercially available PLA fibers are mostly staple fibers and are offered by several companies such as Trevira (Bobingen, Germany), Toray Industries (Chuo, Japan), MiniFibers (Johnson City, United States) and Fiber Innovation Technology (Johnson City, United States). They are sold at the market in different fineness and cut length with tensile strength up to $40 \mathrm{cN} / \mathrm{tex}$ [1].

Due to the biodegradability and biocompatibility [7, 8], PLA fibers are attractive for medical applications like wound dressing and tissue engineering. The fiber is also useful as an eco- and people-friendly alternative to existing textile fibers for industrial and consumer apparel applications such as filtration media, automotive interior fabrics, activewear, shoe linings, and disposable products like diapers and wipes, either at $100 \%$ or in blends with natural fibers such as cotton [2, 9].

PLA is the most commercially available biobased and biodegradable thermoplastic polymer, and is therefore an important substrate for melt-electrospinning technology. Nevertheless only a relatively small number of studies on melt electrospinning of PLA have been carried out. Most PLA-based sub-microfibers have been fabricated using small-scale devices, which are not suitable for sustainable, industrial-scale manufacturing and the majority of fiber diameters achieved have been in the micrometer range [10]. A detailed overview of the state of research on PLA in melt electrospinning is given in the introduction of Chapter 4 (The effect of additives and process parameters 
on the pilot-scale manufacturing of polylactic acid sub-microfibers by melt electrospinning). In comparison to polypropylene, polylactic acid has a higher polarity due to its chemical structure, which has a positive effect on the process functionality. Similar to polypropylene, the properties of PLA can be tailored by the manufacturing parameters [10, 11].

Due to its chemical structure, which in contrast to polypropylene contains a dipole moment, and natural polarity, PLA can be used for melt electrospinning without conductive and viscosity-reducing additives. Taking into account the fiber diameter achieved, mainly in the micrometer range, a transfer of the results of the polypropylene/additive research in melt electrospinning is of great interest and offers the possibility to further reduce the fiber diameter.

In Chapter 4 (The effect of additives and process parameters on the pilot-scale manufacturing of polylactic acid sub-microfibers by melt electrospinning), we therefore investigate the manufacturing of biobased polylactic acid sub-microfibers by melt electrospinning using a single-nozzle laboratory-scale device and the novel 600-nozzle pilot-scale device combined with the conductive and viscosity-reducing additives: sodium stearate, sodium chloride and a polyester-based plasticizer.

Since the added salts could potentially increase the hydrolysis and degradation of the moisture-sensitive polymer, we also investigate the use of the biobased dyes alizarin, hematoxylin and quercetin as conductivity-enhancing additives in PLA laboratory-scale melt electrospinning in Chapter 4 (Biobased dyes as conductive additives to reduce the diameter of polylactic acid fibers during melt electrospinning). 


\section{References}

[1] The Fiber Year Consulting. The Fiber Year 2018 - World Survey on Textiles \& Nonwovens. 2018.

[2] Polymer Properies Database. POLYLACTIC ACID FIBERS 2015. https://polymerdatabase.com/Fibers/PLA.html (acessed 23 February 2020).

[3] NatureWorks. Products 2020. https://www.natureworksllc.com/Products (accessed 23 February 2020).

[4] Endres H-J, Siebert-Raths A. Technische Biopolymere. Rahmenbedingungen, Marktsituation, Herstellung, Aufbau und Eigenschaften.: Hanser Verlag; 2009.

[5] Apex Markets Research. Global PLA Markt 2019-2026. 2020.

[6] Ifbb Hannover. Marktzahlen \& Marktentwicklung biobasierter/bioabbaubarer Kunststoffe. 2018.

[7] Davachi SM, Kaffashi B. Polylactic Acid in Medicine. Polymer-Plastics Technology and Engineering. 2015; 54(9):944-67.

[8] Mogoşanu GD, Grumezescu AM. Natural and synthetic polymers for wounds and burns dressing. International Journal of Pharmaceutics. 2014; 463(2):127-36.

[9] Avinc O, Khoddami A. Overview of Poly(lactic acid) (PLA) Fibre: Part I: Production, Properties, Performance, Environmental Impact, and End-use Applications of Poly(lactic acid) Fibres. Fibre Chemistry. 2009; 41.

[10] Brown T, Dalton P, Hutmacher DW. Melt electrospinning today: an opportune time for an emerging polymer process. Elsevier. 2016; 56.

[11] Zhou H, Green TB, Joo YL. The thermal effects on electrospinning of polylactic acid melts. Polymer (Guildf). 2006; 47. 


\section{Chapter 4}

\section{Polylactic acid in melt electrospinning}

The effect of additives and process parameters on the pilot-scale manufacturing of polylactic acid sub-microfibers by melt electrospinning

K. Koenig, S. Hermanns, J. Ellerkmann, K. Saralidze, F. Langensiepen, G. Seide Textile Research Journal. 2020:0040517520904019.

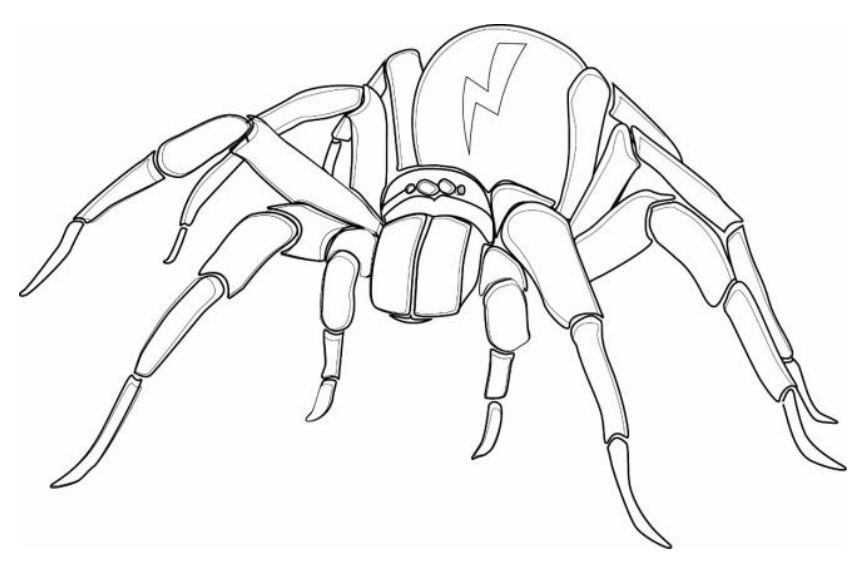




\title{
The effect of additives and process parameters on the pilot- scale manufacturing of polylactic acid sub-microfibers by melt electrospinning
}

\begin{abstract}
Sub-microfibers are polymer filaments less than $1 \mu \mathrm{m}$ in diameter that can be fabricated into highly-flexible materials with a large specific surface area. They are often produced by solution or melt electrospinning. The former is a scalable process that produces thinner fibers but requires hazardous solvents, whereas the latter is more environmentally sustainable due to the absence of solvents but is more challenging to scale up. Here we investigated the manufacturing of biobased polylactic acid (PLA) submicrofibers by melt electrospinning using a single-nozzle laboratory-scale device and a novel 600-nozzle pilot-scale device combined with conductive and viscosity-reducing additives: sodium stearate ( $\mathrm{NaSt})$, sodium chloride $(\mathrm{NaCl})$ and a polyester-based plasticizer. We determined the effect of different additive concentrations on fiber diameter, thermal properties, polymer degradation, and fiber deposition. At the laboratory scale, the minimum average fiber diameter (16 $44 \mu \mathrm{m})$ was accomplished by adding $2 \%(\mathrm{w} / \mathrm{w}) \mathrm{NaCl}$, but a stable spinning process was not achieved and the plasticizer did not reduce the melt viscosity. NaSt was the most effective additive in terms of adapting the material properties of PLA for melt electrospinning, but extensive polymer degradation occurred at higher temperatures and with higher concentrations of the additive. At the pilot scale, the minimum average fiber diameter $(3.77 \mu \mathrm{m})$ was achieved by adding $6 \%(\mathrm{w} / \mathrm{w}) \mathrm{NaSt}$, with a spinneret temperature of $195{ }^{\circ} \mathrm{C}$ and a spin pump speed of $0.5 \mathrm{rpm}\left(0.16 \mathrm{~cm}^{3}\right)$, without further improvements such as the integration of a heating chamber. The smallest single fiber diameter $(1.23 \mu \mathrm{m})$ was achieved at the same conditions but using a spin pump speed of $2 \mathrm{rpm}$. The scaled-up melt-electrospinning device therefore offers significant potential for the production of biobased submicrofibers, bridging the gap between laboratory-scale and pilot-scale manufacturing.
\end{abstract}

\section{Introduction}

Sub-microfibers are filamentous structures with diameters below the microscale range. This confers unique material properties, such as high flexibility and an enormous specific surface area, making such materials suitable for applications in diverse fields including medicine [1-5], energy and electronics [6, 7], filtration and separation [8, 9], and the manufacture of textiles $[10,11]$. Electrospinning has attracted much attention as an efficient method for the fabrication of sub-microfibers because it is simple and inexpensive [12-15]. During the fabrication process, a polymer fluid is stretched by 
electrostatic attraction in the presence of an external static electric field and is deposited on a collector as fine fiber web [11]. Two forms of this method are distinguished: solution electrospinning, where the polymer is initially mixed with a solvent that subsequently evaporates to produce the fibers, and melt electrospinning, where molten polymer is initially held at a high temperature and cools to produce the fibers [12]. Solvent electrospinning is used more widely because it produces thinner fibers and is more scalable, but it often requires toxic solvents. For example, sub-microfibers from polylactic acid (PLA), an environmentally friendly and biodegradable polymer, are usually prepared by solution electrospinning using dichloromethane, chloroform or $\mathrm{N}, \mathrm{N}$-dimethylformamide [16]. The presence of these solvents adds an expensive recovery step to the manufacturing process [17], and the potential carryover of toxic solvents into the final product raises an additional risk for biomedical applications [18, 19]. It is therefore desirable to improve melt-electrospinning technology, aiming to reduce the fiber diameter by overcoming limitations caused by the high temperature, high viscosity and/or low conductivity of the polymer melt compared to the dissolved polymers used in solution electrospinning [20-22]. Another key challenge is the scalability of melt electrospinning, which is currently insufficient for the industrial production of sub-mircofibers [12]. The devices used for melt electrospinning have undergone incremental improvements, and recent innovations such as needleless and multiple-needle configurations have provided a roadmap to overcome the low flow rate (typically in the $\mu \mathrm{g} / \mathrm{h}$ range) and thus increase fiber productivity [12]. Prototypes with umbellate nozzles containing 60 spinnerets can achieve maximum product deposition rates of $\sim 36 \mathrm{~g} / \mathrm{h}[23,24]$. Until recently, the largest multi-needle configuration was a device with 64 nozzles [25], but our latest prototype features 600 nozzles and therefore provides the basis for pilot-scale melt electrospinning $[26,27]$.

The manufacture of fibers in the lower micrometer and sub-micrometer range has been achieved with various polymers, including polyethylene, polypropylene, polycaprolactone, polyurethane, PLA and poly(glycolide-co-lactide),[20, 28-33] combined with diverse melt-electrospinning configurations (but predominantly singlefiber devices). Thinner fibers require further modifications, such as the integration of an additional gas stream [34]. Moreover, additives such as sodium oleate, sodium chloride $(\mathrm{NaCl})$, sodium stearate (NaSt) and plasticizers have been used in previous studies to modify the melt properties of polymers, producing thinner fibers compared to additivefree production [12, 21, 25, 35]. In addition to the need for polymer modification to obtain higher conductivity and lower viscosity, salts are also used as pore-forming agents in the manufacture of scaffolds with increased surface roughness to improve cell adhesion, thereby demonstrating a potential for using melt-electrospun materials in medical applications [36, 37].

PLA is the most commercially available biobased and biodegradable thermoplastic polymer, and is therefore an important substrate for melt-electrospinning technology [16]. PLA fibers produced by melt electrospinning have been modified by adding a plasticizer 
[16, 32], and by adjusting the device during fabrication to facilitate airflow [38] or incorporate laser heating [39]. These approaches led to the production of fibers with diameters in the range $0.2-50 \mu \mathrm{m}$ (Table 4.1) [16, 20, 24, 38-44]. Nevertheless, most PLA-based sub-microfibers have been fabricated using small-scale devices, which are not suitable for sustainable, industrial-scale manufacturing.

Tab. 4.1: Development status of PLA used for melt electrospinning and corresponding fiber diameters.

\begin{tabular}{|c|c|c|c|c|}
\hline PLA type & $\begin{array}{l}\text { Fiber } \\
\text { diameter } \\
(\mu \mathrm{m})\end{array}$ & $\begin{array}{l}\text { Type of } \\
\text { nozzle }\end{array}$ & Technology & Reference \\
\hline NatureWorks 4060D & $3-42$ & Triple & - & [43] \\
\hline NatureWorks 6201D & $19-49$ & Triple & - & [43] \\
\hline Cargill Dow & $0.8-17$ & Single & $\begin{array}{l}\text { Heated spinning } \\
\text { chamber }\end{array}$ & {$[20]$} \\
\hline Cargill Dow & $10-28$ & Single & $\begin{array}{l}\text { Heated spinning } \\
\text { chamber }\end{array}$ & {$[40]$} \\
\hline Cargill Dow & $0.2-3.5$ & Single & $\begin{array}{l}\text { Heated } \\
\text { compressed air }\end{array}$ & [38] \\
\hline Cargill Dow & $0.7-4$ & - & $\begin{array}{l}\text { Rotating disc } \\
\text { collector, laser } \\
\text { heating }\end{array}$ & [39] \\
\hline Cargill Dow & $10-25$ & Single & $\begin{array}{l}\text { Heated spinning } \\
\text { chamber }\end{array}$ & {$[44]$} \\
\hline NatureWorks 2002D & 7.6 & Cone & - & [24] \\
\hline NatureWorks 4060D & $0.9-5$ & - & - & [41] \\
\hline NatureWorks 4060D & $3.1-8.4$ & Triple & - & [42] \\
\hline $\begin{array}{l}\text { Shenzhen Esun } \\
\text { Industrial }\end{array}$ & $0.2-3.5$ & Cone & Compressed air & {$[16]$} \\
\hline
\end{tabular}

Here we set out to fabricate PLA-based sub-microfibers using our 600-nozzle pilot-scale melt-electrospinning device with conductive and viscosity-reducing additives to learn more about the impact of such additives on multi-nozzle electrospinning in the context of industrial production. The spinning trials were carried out without post-heating or additional air flow, which are typically used with smaller-scale devices (Table 4.1), so that system-specific adaptations could be introduced if necessary. Material pre-selection and additive testing were carried out using a conventional single-nozzle meltelectrospinning device before the results were transferred to the pilot-scale setting, 
allowing the comparison of production methods at scale. We investigated the influence of polymer melt viscosity and different concentrations of additives on the fiber diameter, and the effect of different process parameters on the thermal properties of the polymer and its susceptibility to degradation.

\section{Experimental}

\section{Materials}

The material used for melt electrospinning was Ingeo biopolymer 6201D (NatureWorks LLC, Minnetonka, USA), a granular, thermoplastic PLA fiber-grade resin with a melt flow index of $15-30 \mathrm{~g} / 10 \mathrm{~min}$ at $210^{\circ} \mathrm{C}$ and a crystalline melt temperature of $155-170^{\circ} \mathrm{C}$ [45]. We used $\mathrm{NaCl}$ as conductive, NaSt as conductive and viscosity-reducing additive as well as a viscosity-reducing modified polyester (PES). The company Preluna (Ludwigshafen, Germany) advises to use the plasticizer with $1-8 \%(\mathrm{w} / \mathrm{w})$ to achieve an improved flow behavior. The properties of the additives are summarized in Table 4.2.

Tab. 4.2: Properties of additives used in our melt-electrospinning experiments.

\begin{tabular}{|l|l|l|l|l|}
\hline Additives & $\begin{array}{l}\text { Molecular } \\
\text { formula }\end{array}$ & Supplier & $\begin{array}{l}\text { Molecular } \\
\text { weight } \\
\text { (g/mol) }\end{array}$ & State \\
\hline $\begin{array}{l}\text { Sodium stearate }(\mathrm{NaSt}), \\
\geq 99 \%\end{array}$ & $\mathrm{C}_{18} \mathrm{H}_{35} \mathrm{NaO}_{2}$ & Baerlocher & 306.42 & $\begin{array}{l}\text { Solid } \\
\text { (powder) }\end{array}$ \\
\hline $\begin{array}{l}\text { Sodium chloride }(\mathrm{NaCl}), \\
99.5 \%\end{array}$ & $\mathrm{NaCl}$ & Acros Organics & 58.44 & $\begin{array}{l}\text { Solid } \\
\text { (crystals) }\end{array}$ \\
\hline Plasticizer (PES) & - & Preluna & - & $\begin{array}{l}\text { Solid } \\
\text { (granulate) }\end{array}$ \\
\hline
\end{tabular}

All materials were vacuum dried at $60^{\circ} \mathrm{C}$ for $12 \mathrm{~h}$ before compounding and were processed using a ZSK 18 MEGAlab twin-screw extruder (Coperion, Stuttgart, Germany). The temperature of the nine individual heating zones was set to $130-210^{\circ} \mathrm{C}$. The materials were compounded at a screw rotation speed of $250 \mathrm{rpm}$. PLA compounds were produced containing $2 \%, 4 \%, 6 \%$ and $8 \%(\mathrm{w} / \mathrm{w})$ NaSt, $2 \%, 4 \%, 6 \%$ and $8 \%(\mathrm{w} / \mathrm{w})$ $\mathrm{NaCl}$, and $2 \%, 4 \%$ and $6 \%(\mathrm{w} / \mathrm{w})$ PES. The extruded string was granulated using an SP 500 HD strand granulator (Coperion).

\section{Characterization of polymer compounds}

Rheological characterization of the materials, focusing on the frequency-dependent, complex viscosity $\eta^{*}$, was performed using a Discovery HR1 hybrid rheometer (TA Instruments, New Castle, USA). We carried out two frequency sweeps, the first from 628 
to $10 \mathrm{rad} / \mathrm{s}$ and the second from 100 to $0.1 \mathrm{rad} / \mathrm{s}$. For all experiments, we used a $25-\mathrm{mm}$ plate-to-plate geometry. The gap distance was set to $1000 \mu \mathrm{m}$, and the strain amplitude and environment temperature were maintained at $1 \%$ and $200{ }^{\circ} \mathrm{C}$, respectively. To improve the comparability of the results, the viscosity of the different compounds is presented at the same frequency of $10 \mathrm{rad} / \mathrm{s}$. The rheological investigation was performed on both virgin and compounded material, both of which were subjected to the same physical compounding procedure in order to ensure the thermal influence was the same for all samples.

Differential scanning calorimetry (DSC) was carried out using a Q2000 device (TA Instruments), focusing on changes to the glass transition and melting temperatures caused by the presence of additives. Pre-dried samples of 3-5 mg were tested by increasing the starting temperature of $30^{\circ} \mathrm{C}$ at a rate of $10^{\circ} \mathrm{C} / \mathrm{min}$ under nitrogen at a flow rate of $50 \mathrm{ml} / \mathrm{min}$ until the temperature reached $250^{\circ} \mathrm{C}$. Data were visualized using Universal Analysis Software (TA Instruments).

Thermogravimetric analysis (TGA) was carried out using a Q5000 device (TA Instruments). The samples $\left(10-15 \mathrm{mg}\right.$ ) were heated at a constant rate of $10^{\circ} \mathrm{C} / \mathrm{min}$ under nitrogen at a flow rate of $50 \mathrm{ml} / \mathrm{min}$ until the temperature reached $700{ }^{\circ} \mathrm{C}$. In any case the DSC and TGA measurement was repeated three times. The initial temperature of degradation and the weight share (or mass proportion, which is defined as the mass of a component divided by the total mass of the mixture, multiplied by $100 \%$ ) at $200{ }^{\circ} \mathrm{C}$ were determined, and thermogravimetric curves were plotted using Universal Analysis Software.

The TGA tests did not incorporate a time factor (more specifically, a dwell time) during the melt-electrospinning process, so we investigated the degradation of the polymers after compounding and rheological characterization to determine the influence of prolonged exposure to a temperature of $200^{\circ} \mathrm{C}$. The tests were carried out by gel permeation chromatography (GPC) using a 1260 Infinity GPC/SEC System (Aligent Technologies, Santa Clara, USA) with hexafluor-2-isopropanol (HFIP) containing $0.19 \%$ sodium trifluoroacetate as the mobile phase flowing at a rate of $0.33 \mathrm{ml} / \mathrm{min}$. The polymer sample $(5 \mathrm{mg}$ ) was dissolved in HFIP for $2 \mathrm{~h}$ before injection into a PFG combination medium column with $7 \mu \mathrm{m}$ particle size (Polymer Standards Service, Mainz, Germany). The relative molecular weight and polydispersity were determined using refractive index detectors following calibration with a polymethacrylate standard $\left(1.0 \times 10^{5} \mathrm{~g} / \mathrm{mol}\right)$.

\section{Melt-electrospinning equipment}

To evaluate the general processability of the PLA compounds and their fiber formation characteristics, we used an in-house laboratory-scale single-fiber melt-electrospinning device with five major components: temperature controller, high-voltage power supply, heating elements, syringe pump, and collector (Figure 4.1). The device was fitted with JCS-33A temperature process controllers (Shinko Technos, Osaka, Japan) and PT 100 platinum thermocouples (Omega Engineering, Deckenpfron, Germany) to control the 
melting temperature. The temperature was set to $190{ }^{\circ} \mathrm{C}$ for the NaSt compounds and $250^{\circ} \mathrm{C}$ for the PES and $\mathrm{NaCl}$ compounds. We used a $\mathrm{KNH} 65$ high-voltage generator (Eltex-Elektrostatik, Weil am Rhein, Germany) with a voltage range of 6-60 kV. During the melt-electrospinning experiments, the voltage was kept constant at $50 \mathrm{kV}$ for the single-fiber device. A positive voltage was applied to the collector while grounding the spinneret. A flat aluminum plate $(6 \mathrm{~cm})$ was used as a collector. The distance between the spinneret and collector was set at $10 \mathrm{~cm}$ for all tests. An 11 Plus spin pump (Harvard Apparatus, Cambridge, USA) was used with a constant delivery rate of $4 \mathrm{ml} / \mathrm{h}$. The spinneret was constructed from a 2-ml glass syringe (Poulten \& Graf, Wertheim, Germany) with a nozzle orifice of $1 \mathrm{~mm}$.

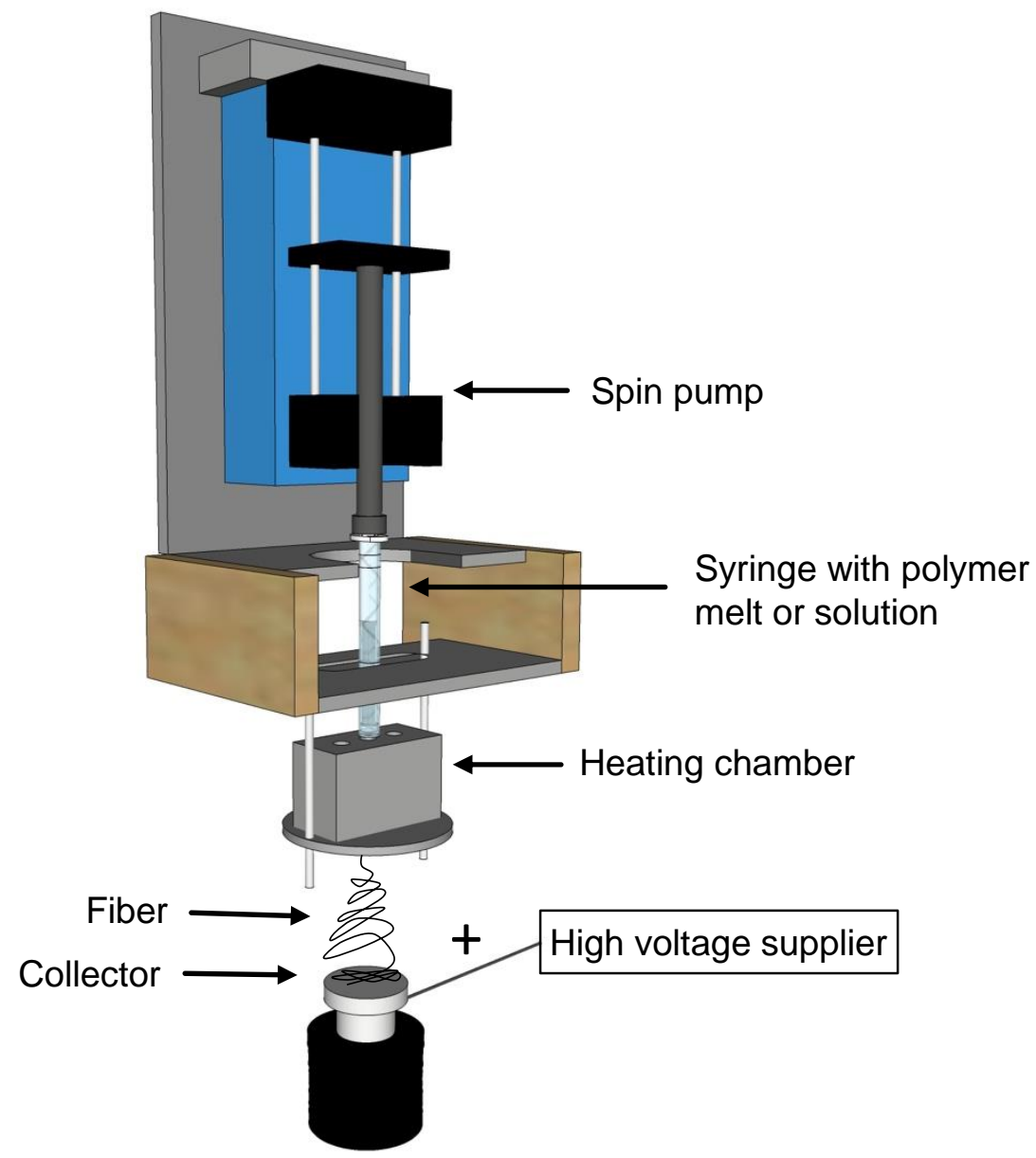

Fig. 4.1: Single-fiber melt-electrospinning setup.

To scale up the melt-electrospinning process beyond current state-of-the-art technologies, we used a newly-developed prototype pilot-scale setup including a spinneret with 600 nozzles, each $0.3 \mathrm{~mm}$ in diameter and spaced at $8 \mathrm{~mm}$ intervals [27]. The new device development is based on the concept idea of Christoph Hacker et al. $[46,47]$, whereby a nozzle revision to reduce dwell times, a more efficient heating system, and a new collector design and fiber deposition concept were integrated [27]. A schematic of our pilot-scale melt-electrospinning device is shown in Figure 4.2. A 
constant supply of polymer melt was ensured by a speed-adjustable single-screw extruder with three heating zones based on integrated heating elements and a spinning pump. An aluminum collector with an uneven surface was used instead of a conventional plate collector. The adjustable collector pins can be placed $8 \mathrm{~mm}$ apart. A narrow and offset pin order with a diagonal distance of $2.6 \mathrm{~cm}$ was used. With the nozzle/collector pairing installed in the melt-electrospinning prototype, nonwovens up to $340 \mathrm{~mm}$ in width could be produced continuously. The distance between the collector and the nozzle plate was set to $11 \mathrm{~cm}$. A positive voltage of $60 \mathrm{kV}$ was applied to the collector. As with the laboratory-scale system, the voltage was applied to the collector with a simultaneous grounding of the spinneret.

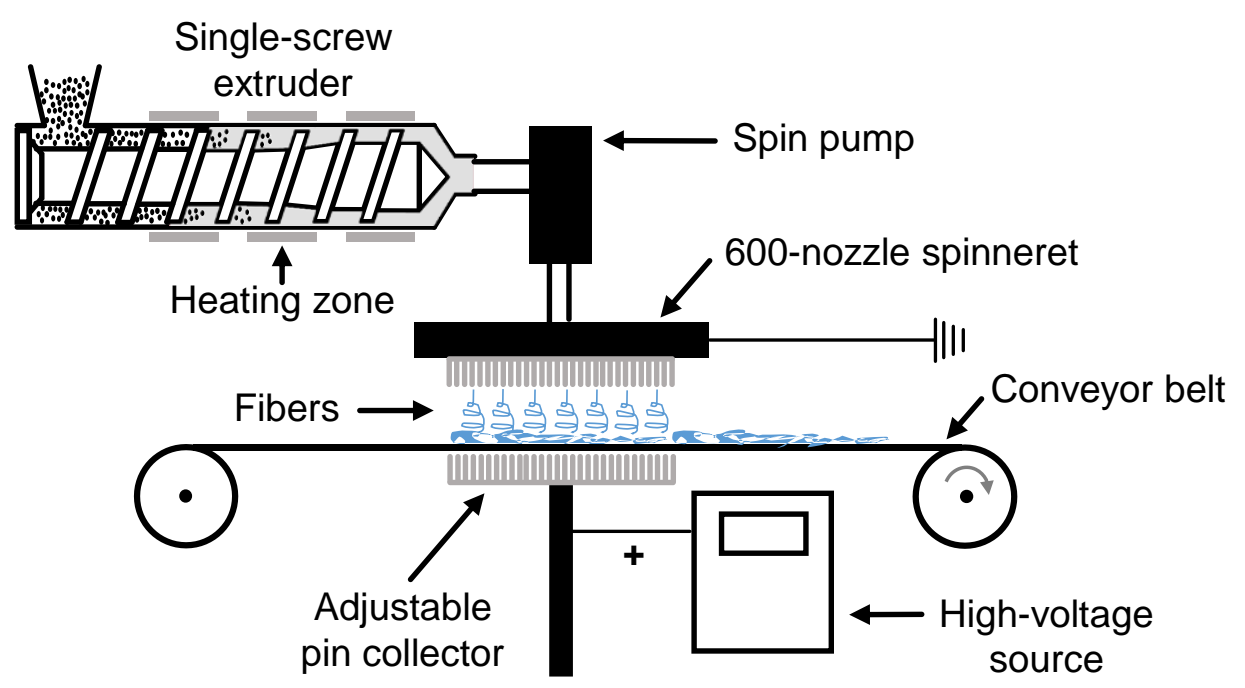

Fig. 4.2: Schematic of the pilot-scale device used for melt electrospinning.

\section{Characterization of fibers}

Fiber diameters were determined by reflected light microscopy using a DM4000 M instrument (Leica Microsystems $\mathrm{GmbH}$, Wetzlar Germany) at 100-200× magnification, and images were captured using Leica Application Suite software. In each case the fiber diameter was measured fifty times at different sections in the nonwoven sample. The surface morphology, formation and distribution of fibers was investigated by scanning electron microscopy (SEM) using an XL 30 device (FEl/Koninklijke Philips, Amsterdam, The Netherlands) with an accelerating voltage of $10 \mathrm{kV}$. The samples were attached to a stub with double-sided carbon tape and sputtered with gold prior to SEM to produce a conductive surface. 


\section{Results and discussion}

\section{Effects on viscosity}

We investigated the effect of different additive concentrations initially using the laboratory-scale melt-electrospinning device. The complex viscosity curves of virgin PLA and PLA compounds with increasing weight ratios of the additives PES, $\mathrm{NaSt}$ and $\mathrm{NaCl}$ are shown in Figure 4.3, corresponding to a frequency of $10 \mathrm{rad} / \mathrm{s}$ and a set temperature of $200{ }^{\circ} \mathrm{C}$. The time until the point of complex viscosity is recorded for a frequency of $10 \mathrm{rad} / \mathrm{s}$ differs over all compounds by a maximum of $70 \mathrm{sec}$ (Appendix: Fig. A1). A distinction of the thermal degradation behavior due to the longer exposure time is not made here, since the time factor is estimated to be small compared to the actual spinning process and the shear forces occurring.

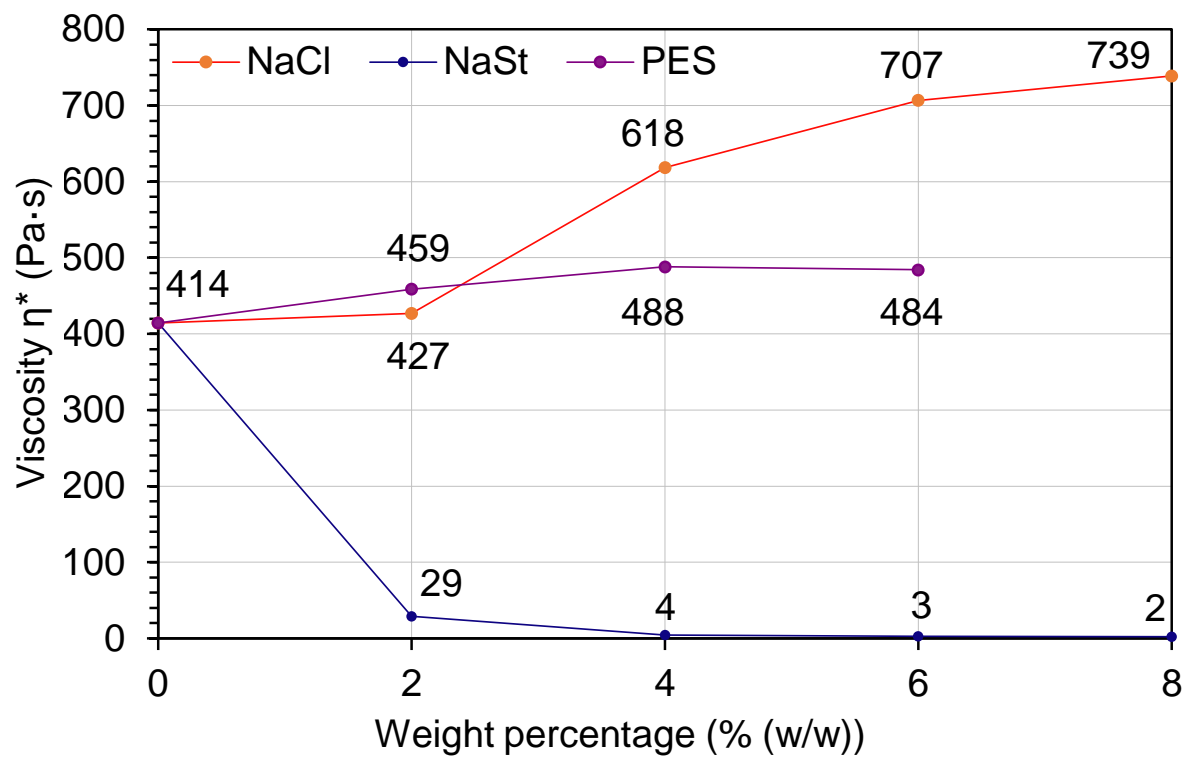

Fig. 4.3: Complex viscosity of PLA and PLA compounds with increasing weight ratios of PES, NaSt and $\mathrm{NaCl}$ determined at a frequency of $10 \mathrm{rad} / \mathrm{s}$ and a temperature of $200^{\circ} \mathrm{C}$.

The addition of NaSt caused a substantial reduction in the viscosity of the melt. The presence of $2 \%(w / w)$ NaSt reduced the viscosity of pure PLA (414 Pa.s) to $29 \mathrm{~Pa} \cdot \mathrm{s}$, a difference of $93 \%$ (385 Pa.s). Increasing the weight ratio further had a smaller but still

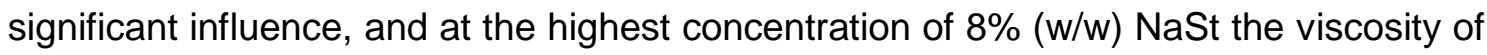
the melt was only $2 \mathrm{~Pa} \cdot \mathrm{s}$. NaSt acts as a lubricant between polymer chains, so a lower melt viscosity was anticipated [48].

In contrast to $\mathrm{NaSt}$, the addition of $\mathrm{NaCl}$ and PES caused the melt viscosity of PLA to increase. In the case of PES, the increase was contrary to expectations because the polyester-based plasticizer is specifically designed to reduce the viscosity of polyesters, polypropylene, polyamides and PLA. A small reduction in viscosity was observed at 
$6 \%(\mathrm{w} / \mathrm{w})$ PES, so a larger weight ratio of the additive may be necessary to achieve a significant effect at our measurement temperature of $200^{\circ} \mathrm{C}$. $\mathrm{NaCl}$ did not dissolve in the polymer, so an increase in viscosity was anticipated. Nevertheless, $\mathrm{NaCl}$ can reduce the fiber diameter during melt electrospinning by increasing the electrical conductivity, which can compensate for the increase in viscosity [49].

\section{Thermal properties}

The glass transition and melting temperatures of PLA and its compounds were determined by DSC, and the presence of additives appeared to have little effect on these values (Table 4.3)

Tab. 4.3: Glass transition and melting temperatures of PLA and its compounds.

\begin{tabular}{|c|c|c|}
\hline Material & $\begin{array}{l}\text { Glass transition } \\
\text { temperature } \\
\left({ }^{\circ} \mathrm{C}\right)\end{array}$ & $\begin{array}{l}\text { Melting temperature } \\
\left({ }^{\circ} \mathrm{C}\right)\end{array}$ \\
\hline NatureWorks PLA 6201D & $64.8 \pm 0.2$ & $166.8 \pm 0.3$ \\
\hline$P L A+2 \%(w / w)$ PES & $61.7 \pm 0.4$ & $167.9 \pm 0.2$ \\
\hline$P L A+4 \%(w / w)$ PES & $61.5 \pm 0.2$ & $167.9 \pm 0.3$ \\
\hline$P L A+6 \%(w / w)$ PES & $61.2 \pm 0.3$ & $168.2 \pm 0.5$ \\
\hline $\mathrm{PLA}+2 \%(w / w) \mathrm{NaCl}$ & $59.8 \pm 0.9$ & $167.6 \pm 0.7$ \\
\hline $\mathrm{PLA}+4 \%(\mathrm{w} / \mathrm{w}) \mathrm{NaCl}$ & $61.0 \pm 0.6$ & $167.1 \pm 0.4$ \\
\hline $\mathrm{PLA}+6 \%(w / w) \mathrm{NaCl}$ & $61.8 \pm 0.7$ & $167.0 \pm 0.8$ \\
\hline $\mathrm{PLA}+8 \%(w / w) \mathrm{NaCl}$ & $64.2 \pm 0.7$ & $166.2 \pm 0.1$ \\
\hline $\mathrm{PLA}+2 \%(w / w) \mathrm{NaSt}$ & $64.6 \pm 0.5$ & $166.7 \pm 0.5$ \\
\hline $\mathrm{PLA}+4 \%(\mathrm{w} / \mathrm{w}) \mathrm{NaSt}$ & $62.5 \pm 0.8$ & $164.4 \pm 1.2$ \\
\hline $\mathrm{PLA}+6 \%(\mathrm{w} / \mathrm{w}) \mathrm{NaSt}$ & $64.1 \pm 0.2$ & $165.8 \pm 0.7$ \\
\hline $\mathrm{PLA}+8 \%(w / w) \mathrm{NaSt}$ & $63.5 \pm 1.1$ & $165.5 \pm 0.9$ \\
\hline
\end{tabular}

As an example, the thermographs in Figure 4.4 show the first heating cycle of virgin PLA and its compounds containing different weight ratios of NaSt. A double peak is present in the thermographs of the semi-crystalline PLA as well as the compounds, which is consistent with a crystallization temperature that supports the simultaneous formation of $\alpha^{\prime}$ and $\alpha$ type crystals [50]. 


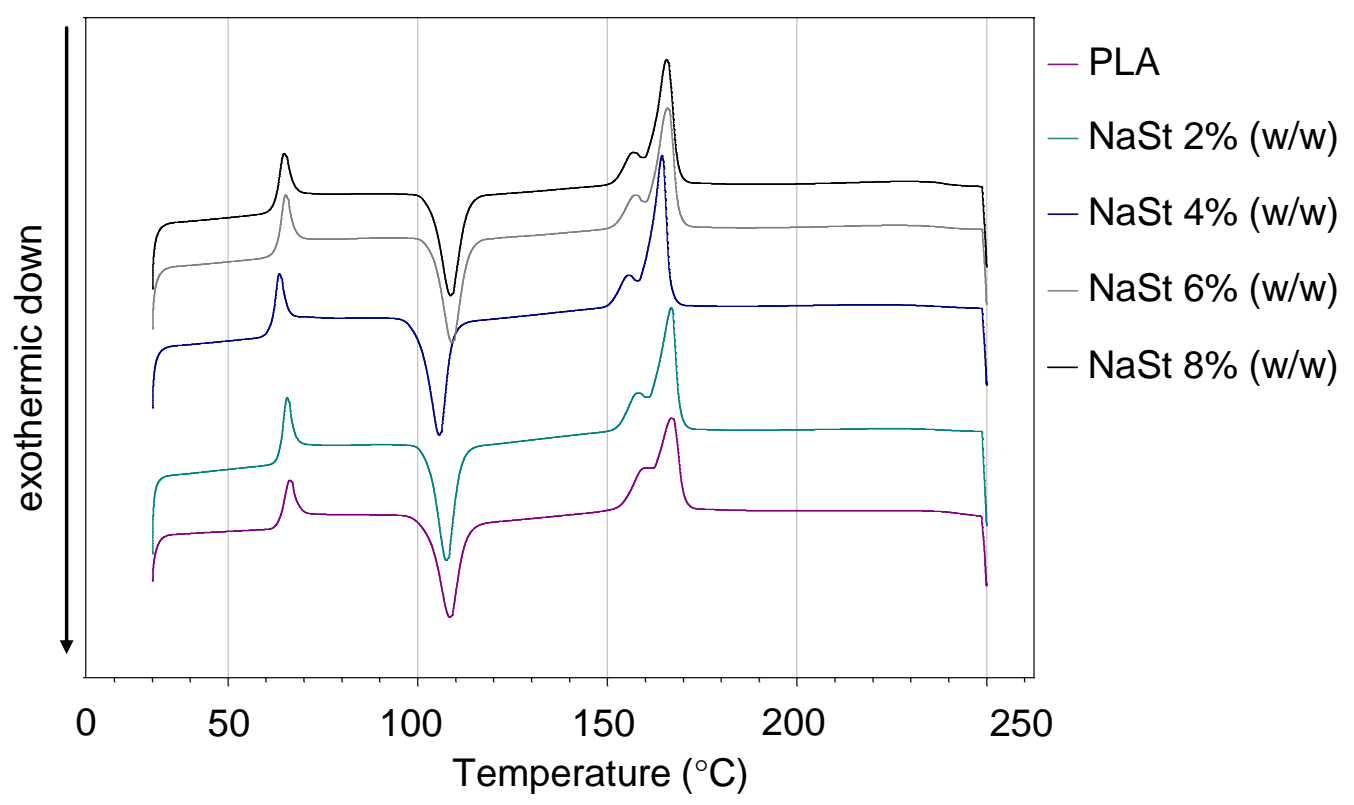

Fig. 4.4: Thermographs of virgin PLA and PLA containing 2-8\% (w/w) NaSt.

The decomposition temperatures of virgin PLA, the additives and compounds were investigated by TGA, and the initial degradation temperatures and weight shares at $200^{\circ} \mathrm{C}$ are summarized in Table 4.4. The decomposition temperature for pure PES $\left(369.2^{\circ} \mathrm{C}\right)$ was $39.4^{\circ} \mathrm{C}$ higher than that of virgin PLA $\left(329.8^{\circ} \mathrm{C}\right)$. The decomposition temperature was therefore higher for compounds containing larger amounts of PES, but the materials began to decompose earlier than the base materials PLA and PES and thus the degradation temperature was lower. The same behavior was observed for the weight share at $200{ }^{\circ} \mathrm{C}$, and similar profiles were apparent for the compounds containing $\mathrm{NaCl}$. Nevertheless, there was no overt temperature fluctuation among the compounds. $\mathrm{NaCl}$ did not show any significant degradation at temperatures up to $700{ }^{\circ} \mathrm{C}$ whereas PES degraded almost completely in this range, but all compounds still contained a weight share at $700^{\circ} \mathrm{C}$. The lowest decomposition temperatures were observed for $\mathrm{NaSt}$ (Figure 4.5). A small loss of weight was observed starting at $208.6^{\circ} \mathrm{C}$, but massive loss and degradation was apparent at $417.2^{\circ} \mathrm{C}$. The initial degradation temperature of the PLA compounds containing NaSt decreased with larger weight ratios of $\mathrm{NaSt}$, which contrasts with the behavior of the PLA compounds containing PES and $\mathrm{NaCl}$. The PLA compounds containing NaSt showed negligible weight loss at $200^{\circ} \mathrm{C}$, as similarly observed for compounds containing the other additives. 
Tab. 4.4: Initial degradation temperatures and weight shares at $200{ }^{\circ} \mathrm{C}$ for PLA and its compounds.

\begin{tabular}{|c|c|c|}
\hline Material & $\begin{array}{l}\text { Initial degradation temperature } \\
\left({ }^{\circ} \mathrm{C}\right)\end{array}$ & $\begin{array}{l}\text { Weight share } \\
\text { (\%) }\end{array}$ \\
\hline NatureWorks PLA 6201D & $329.8 \pm 2.3$ & 99.8 \\
\hline PES & $369.2 \pm 1.7$ & 99.8 \\
\hline$P L A+2 \%(w / w)$ PES & $310.2 \pm 3.1$ & 99.5 \\
\hline$P L A+4 \%(w / w)$ PES & $323.4 \pm 2.7$ & 99.5 \\
\hline$P L A+6 \%(w / w) P E S$ & $327.8 \pm 1.6$ & 99.5 \\
\hline $\mathrm{NaCl}$ & $393.3 \pm 0.9$ & 99.9 \\
\hline $\mathrm{PLA}+2 \%(w / w) \mathrm{NaCl}$ & $312.9 \pm 3.4$ & 99.5 \\
\hline $\mathrm{PLA}+4 \%(w / w) \mathrm{NaCl}$ & $306.7 \pm 2.8$ & 99.6 \\
\hline $\mathrm{PLA}+6 \%(w / w) \mathrm{NaCl}$ & $311.6 \pm 2.7$ & 99.7 \\
\hline $\mathrm{PLA}+8 \%(w / w) \mathrm{NaCl}$ & $310.7 \pm 3.2$ & 99.6 \\
\hline $\mathrm{NaSt}$ & $417.5 \pm 1.5$ & 98.2 \\
\hline $\mathrm{PLA}+2 \%(w / w) \mathrm{NaSt}$ & $277.7 \pm 1.6$ & 99.2 \\
\hline $\mathrm{PLA}+4 \%(w / w) \mathrm{NaSt}$ & $269.8 \pm 2.0$ & 99.0 \\
\hline $\mathrm{PLA}+6 \%(\mathrm{w} / \mathrm{w}) \mathrm{NaSt}$ & $263.6 \pm 0.8$ & 99.1 \\
\hline $\mathrm{PLA}+8 \%(w / w) \mathrm{NaSt}$ & $255.1 \pm 1.3$ & 98.2 \\
\hline
\end{tabular}

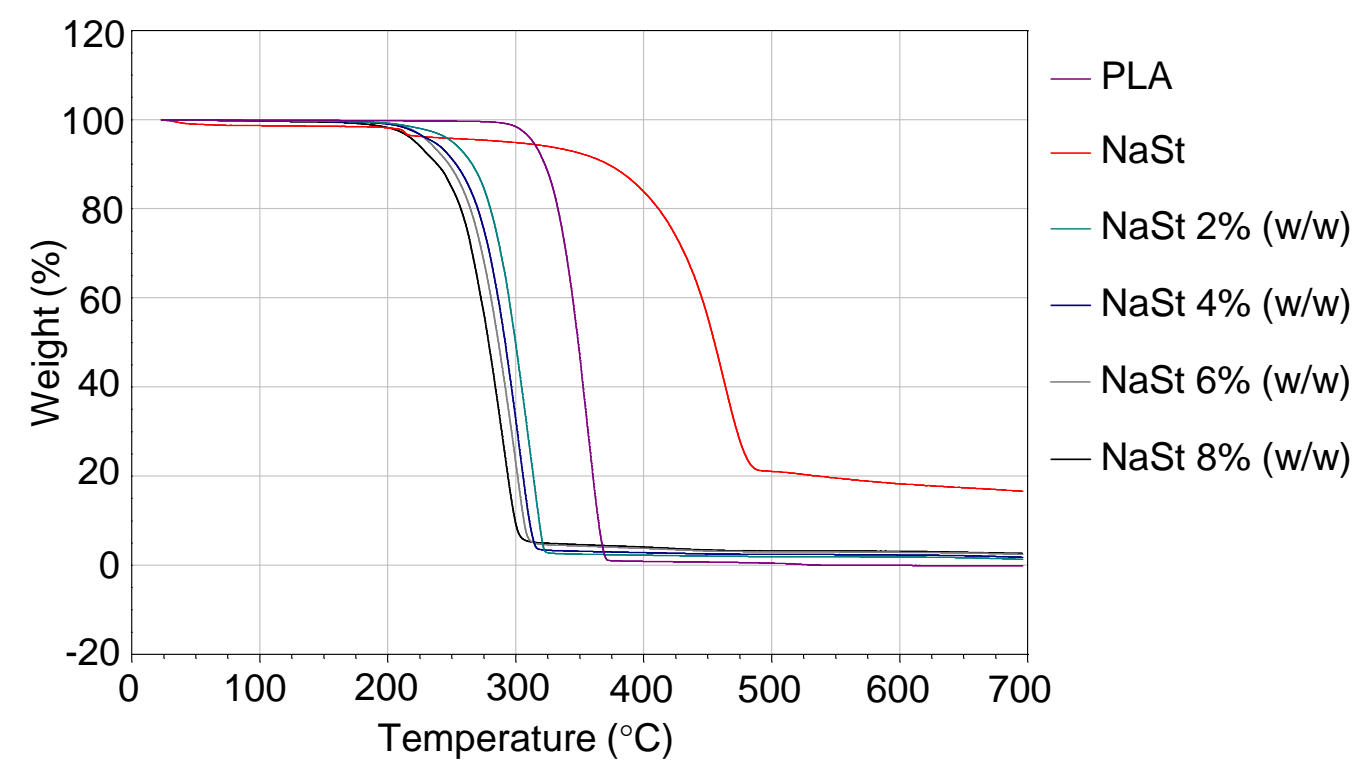

Fig. 4.5: TGA curves of virgin PLA and PLA containing 2-8\% (w/w) NaSt. 
Because no time factor was taken into account for the TGA measurements, the effects of heat input at $200{ }^{\circ} \mathrm{C}$ on the molecular distribution and weight after compounding and rheological characterization were investigated by GPC. For PES and $\mathrm{NaCl}$, no significant change in molar mass was observed after compounding or rheological characterization (Appendix: Fig. A2 and Fig. A3), but this was not the case for PLA and its compounds containing NaSt (Figure 4.6).

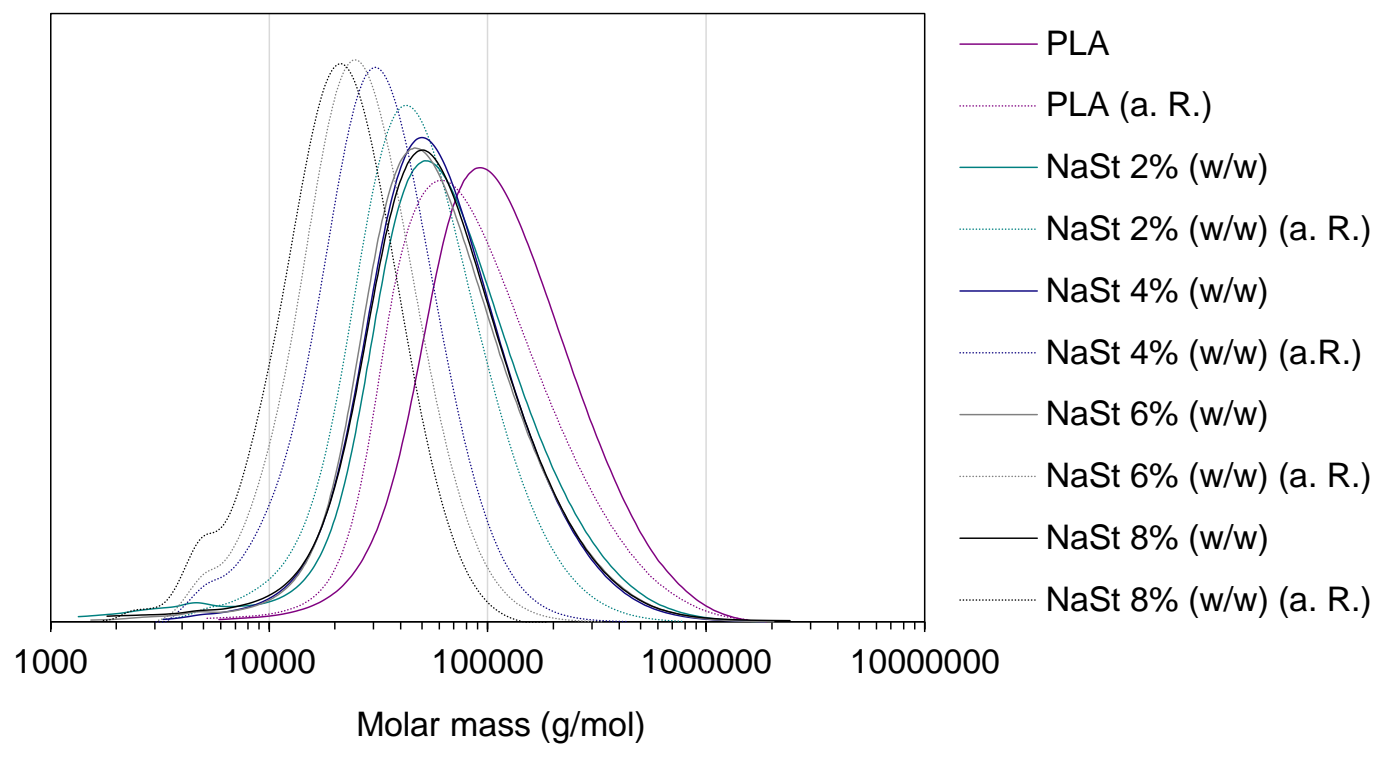

Fig. 4.6: GPC curves of virgin PLA and PLA containing 2-8\% (w/w) NaSt after compounding and after the time influence of the rheometer test (a.R.).

In contrast to the TGA data, which indicated no degradation at temperatures below $250^{\circ} \mathrm{C}$, GPC revealed that compounding of the virgin PLA and compounds containing $\mathrm{NaSt}$ with the twin screw extruder led to a shift towards lower molar masses, exacerbated by the time influence during rheological characterization. When determining the temperature for the spinning process, it should be taken into account that a material degradation at $200^{\circ} \mathrm{C}$ could be detected after the time influence of the rheology measurement. The process window should therefore be chosen above the melting point and, if processing allows, below $200{ }^{\circ} \mathrm{C}$ to prevent premature material degradation. Further tests to define the lower temperature limit of degradation and influence of the actual shear forces in the spinning process in dependence of the machine design were not performed here.

\section{Fiber diameters and distribution}

The processability of the materials and the influence of additives on the fiber diameter were investigated by producing fibers using the single-fiber melt-electrospinning device. The formation of a Taylor cone followed by typical fiber deposition was observed at a temperature of $190^{\circ} \mathrm{C}$ for the NaSt compounds and at $250{ }^{\circ} \mathrm{C}$ for pure PLA and PES. The compounds containing NaSt and PES supported constant fiber formation, whereas undissolved $\mathrm{NaCl}$ crystals were attracted by the high voltage field, resulting in an 
unstable melt flow that prevented continuous fiber deposition for compounds containing $\mathrm{NaCl}$. Furthermore, the compounds containing $6 \%$ and $8 \%(\mathrm{w} / \mathrm{w}) \mathrm{NaCl}$ could not be pressed through the syringe due to high frictional forces. At temperatures exceeding $190{ }^{\circ} \mathrm{C}$, materials containing NaSt could not be processed because degradation had already begun and the weakened melt could only produce droplets. To achieve a better comparison with the NaSt compounds and to investigate the influence of temperature, a virgin PLA sample was collected at $190^{\circ} \mathrm{C}$. Given that no whipping or stretching of the PLA melt was visible, a larger standard deviation was observed because a constant process could not be achieved (Figure 4.7).

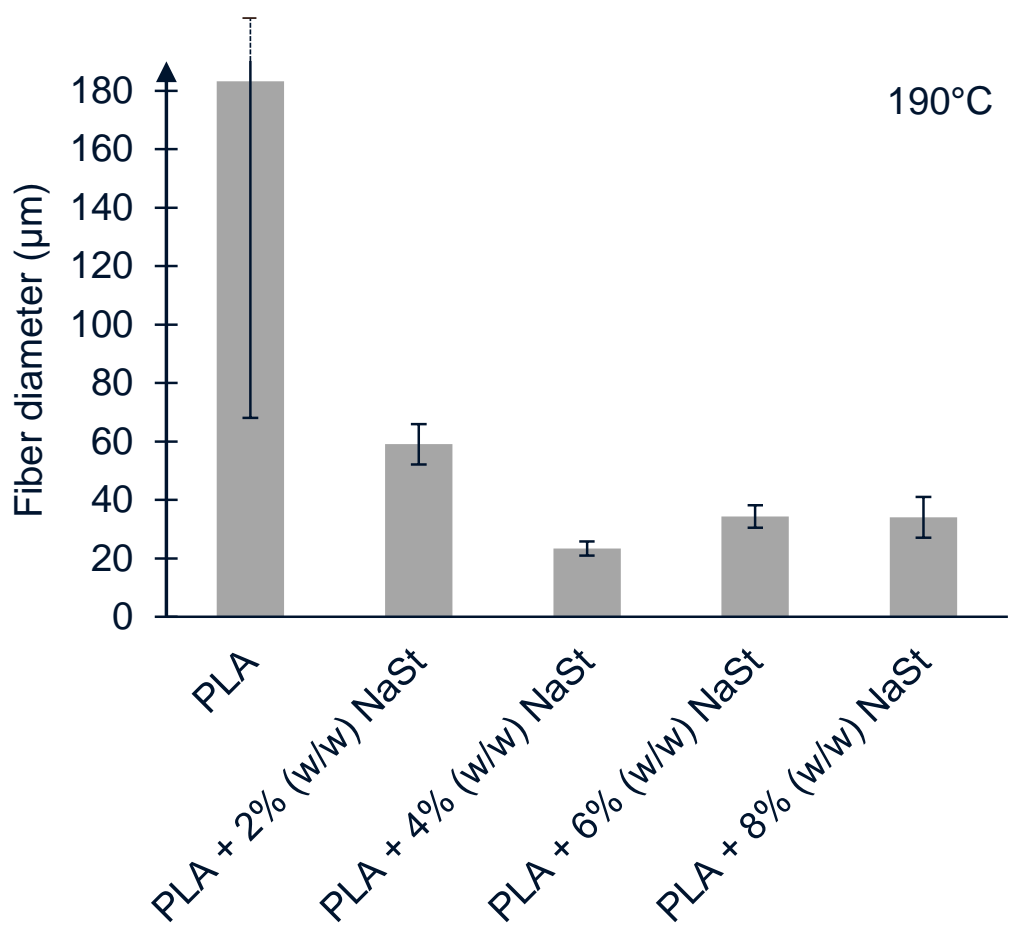

Fig. 4.7: Fiber diameters of PLA and PLA compounds containing NaSt produced at $190^{\circ} \mathrm{C}$ using the single-fiber melt-electrospinning device.

The addition of $2 \%$ or $4 \%(\mathrm{w} / \mathrm{w})$ NaSt reduced the fiber diameter significantly from 183.24 to $23.34 \mu \mathrm{m}$, which is the lowest average fiber diameter we achieved in the laboratory-scale device. The $4 \%(\mathrm{w} / \mathrm{w})$ NaSt compound also produced the lowest single fiber diameter $(19.50 \mu \mathrm{m})$ and the lowest standard deviation of $2.43 \mu \mathrm{m}$. In the presence of $6 \%$ and $8 \%(\mathrm{w} / \mathrm{w}) \mathrm{NaSt}$, the average fiber diameter increased again to 34.33 and $34.01 \mu \mathrm{m}$, respectively. This weight ratio of additive reduced the viscosity and melt strength of the material too much, promoting faster material flow under gravity and thus allowing less time for whipping instabilities to occur, ultimately reducing the whipping motion and inhibiting the elongation and strengthening of the jet, resulting in thicker fiber diameters.

The fiber diameters and standard deviations for PLA and its PES and $\mathrm{NaCl}$ compounds at $250^{\circ} \mathrm{C}$ are shown in Figure 4.8. Despite the increase in viscosity at $200^{\circ} \mathrm{C}$, the fiber 
diameter could be reduced at a process temperature of $250^{\circ} \mathrm{C}$ by adding $2 \%(w / w)$ PES. Nevertheless, the fiber diameter increased again in the presence of $4 \%(w / w)$ PES. The influence of PES on the spinning process and thus the fiber diameter could not be determined after the trials because no clear tendencies were observed. The analysis of the $\mathrm{NaCl}$ compounds revealed that the increase in conductivity has a more significant effect on the fiber diameter than reducing the viscosity. $\mathrm{NaCl}$ has a lower molar weight than $\mathrm{NaSt}$ and more $\mathrm{NaCl}$ molecules are therefore incorporated into PLA compounds at a constant weight ratio, resulting in a larger number of ions on the prerequisite that the salt fully dissolves. Rheometry shows that this is not the case for concentrations above $2 \%(\mathrm{w} / \mathrm{w}) \mathrm{NaCl}$, but even if $2 \%(\mathrm{w} / \mathrm{w}) \mathrm{NaCl}$ dissolves, there are likely more sodium ions compared to sodium stearate. Although the viscosity of compounds containing $2 \%$ and $4 \%(\mathrm{w} / \mathrm{w}) \mathrm{NaCl}$ was higher than that of virgin PLA, the average fiber diameter was much smaller, reaching a minimum of $16.45 \mu \mathrm{m}$ in the presence of $2 \%(\mathrm{w} / \mathrm{w}) \mathrm{NaCl}$.

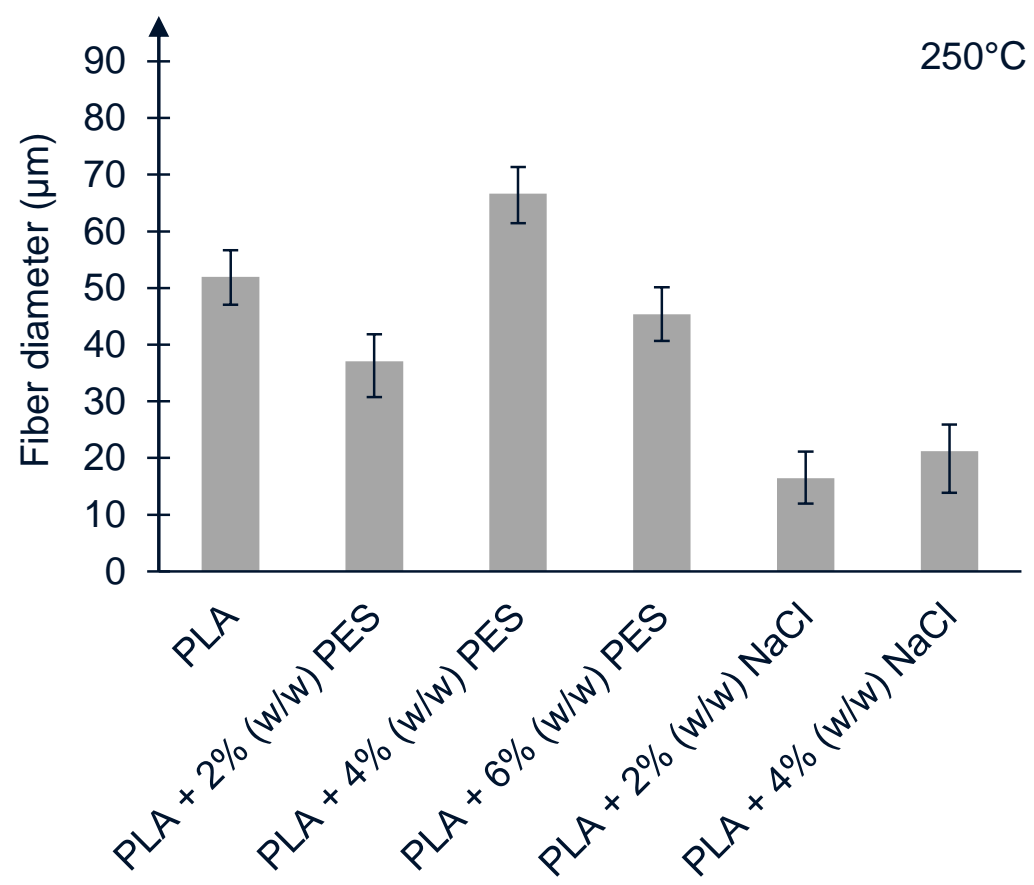

Fig. 4.8: Fiber diameters of PLA and PLA compounds containing $\mathrm{NaCl}$ and PES produced at $250^{\circ} \mathrm{C}$ using the single-fiber melt-electrospinning device.

Figure 4.9 shows SEM images of PLA fibers containing 4\% (w/w) NaSt (Figure 4.9a, b) and $2 \%(\mathrm{w} / \mathrm{w}) \mathrm{NaCl}$ (Figure 4.9c, d) produced using the single-nozzle device. The NaSt fibers are distributed more homogeneously, whereas the $\mathrm{NaCl}$ fibers appear as clumped 'beads' in which crystals of $\mathrm{NaCl}$ are clearly embedded. 

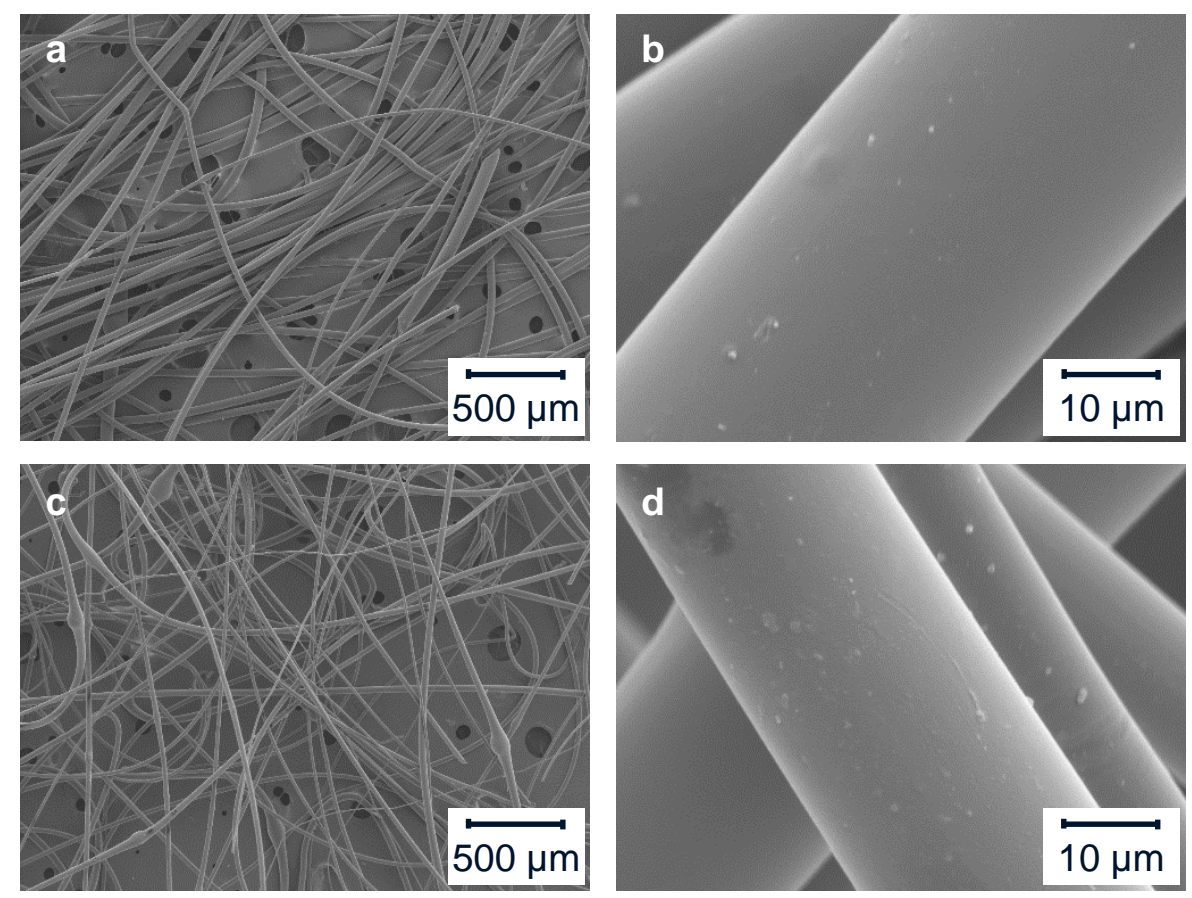

Fig. 4.9: SEM images of PLA fibers produced with the single-nozzle electrospinning device. (a, b) PLA containing 4\% (w/w) NaSt: (a) $\times 100$, (b) $\times 5000$. (c, d) PLA fibers containing $4 \%(\mathrm{w} / \mathrm{w}) \mathrm{NaCl}$ : (c) $\times 100$, (d) $\times 5000$.

Given that NaSt significantly reduced the fiber diameter and also achieved a stable melt-electrospinning process, materials containing 2-8\% (w/w) NaSt were tested using the pilot-scale melt-electrospinning device at spinning temperatures of 195 and $205^{\circ} \mathrm{C}$ and at different spin pump speeds. As discussed above, higher weight ratios of $\mathrm{NaSt}$ promoted the degradation of PLA compounds, so the compound containing $8 \%(\mathrm{w} / \mathrm{w})$ NaSt could not be processed at $195{ }^{\circ} \mathrm{C}$ with a longer dwell time of up to $50 \mathrm{~min}$ because droplets of the decomposing material prevented the constant formation of fibers. The average fiber diameters (and standard deviations) achieved using compounds containing 2-6\% (w/w) NaSt at 195 and $205^{\circ} \mathrm{C}$ are shown in Figure 4.10, which also compares spin pump speeds of $10 \mathrm{rpm}\left(3.2 \mathrm{~cm}^{3}\right)$ and $5 \mathrm{rpm}\left(1.6 \mathrm{~cm}^{3}\right)$. 


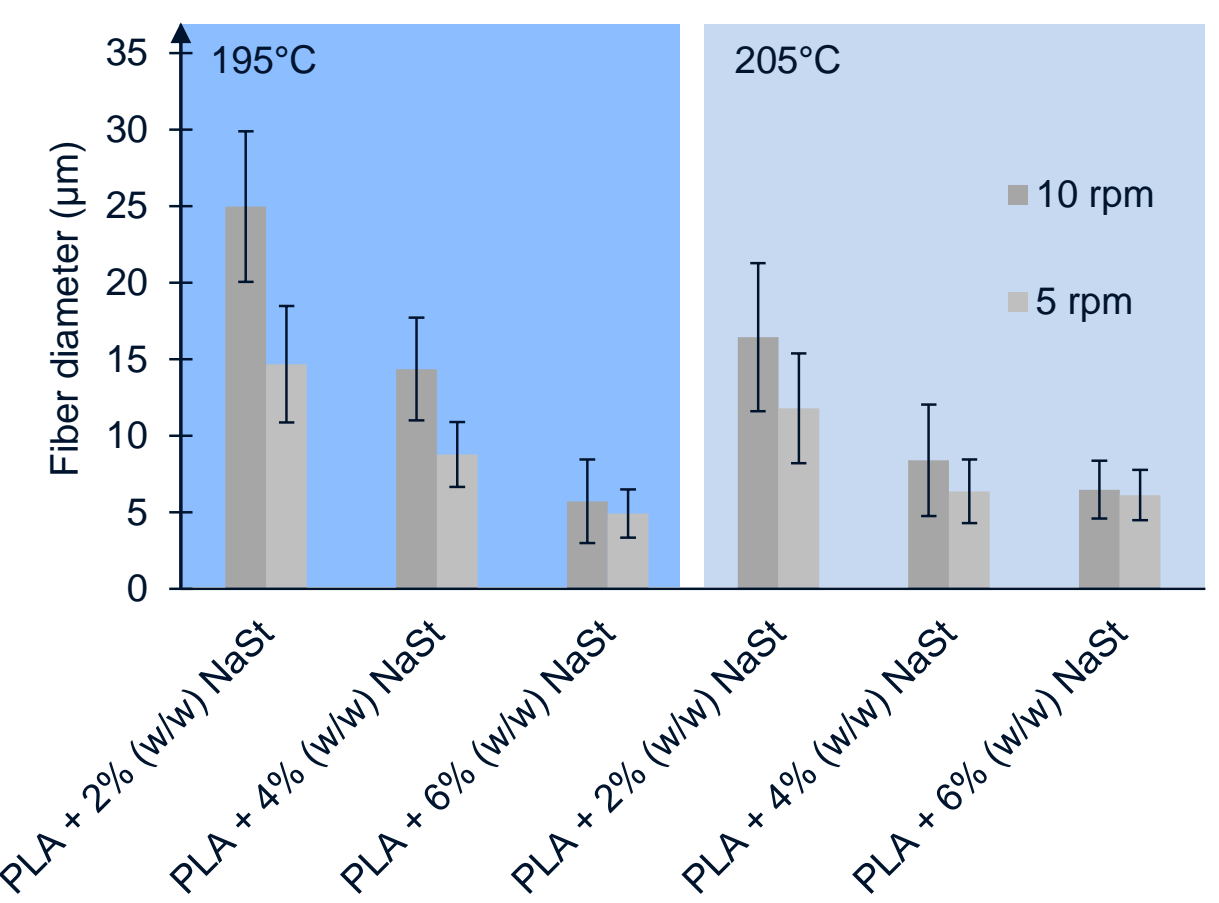

Fig. 4.10: Diameters of PLA fibers containing 2-6\% (w/w) NaSt produced at 195 and $205{ }^{\circ} \mathrm{C}$ with spin pump speeds of 10 and $5 \mathrm{rpm}$ using the 600 -nozzle meltelectrospinning prototype.

Reducing the nozzle diameter from $1 \mathrm{~mm}$ on the laboratory-scale device to $0.3 \mathrm{~mm}$ in the pilot-scale spinneret generally achieved a significant reduction in fiber diameter as expected from previous publications. However, a direct comparison of the devices must be considered critically due to the different polymer melt flow, shear forces and heat distribution. The slower spin pump speed also reduced the fiber diameter by over one third at $195^{\circ} \mathrm{C}$, although the effect was less noticeable at $205^{\circ} \mathrm{C}$. The fiber diameter (and standard deviation) also decreased in the presence of higher weight ratios of $\mathrm{NaSt}$. Furthermore, at least in the case of compounds containing $2 \%$ or $4 \%(\mathrm{w} / \mathrm{w}) \mathrm{NaSt}$, the fiber diameter was also reduced by the lower viscosity at higher process temperatures. For PLA containing 6\% (w/w) NaSt the effect of temperature was minimal, as observed with the single-nozzle syringe. The degradation that occurs and thus the increase in mass flow must be taken into account, which results in slightly higher average fiber diameters at $205^{\circ} \mathrm{C}$. This effect was even more apparent when PLA containing $6 \%(\mathrm{w} / \mathrm{w})$ NaSt was processed by further reducing the spin pump speed to $2 \mathrm{rpm}\left(0.64 \mathrm{~cm}^{3}\right)$ and then $0.5 \mathrm{rpm}\left(0.16 \mathrm{~cm}^{3}\right)$ (Figure 4.11). 


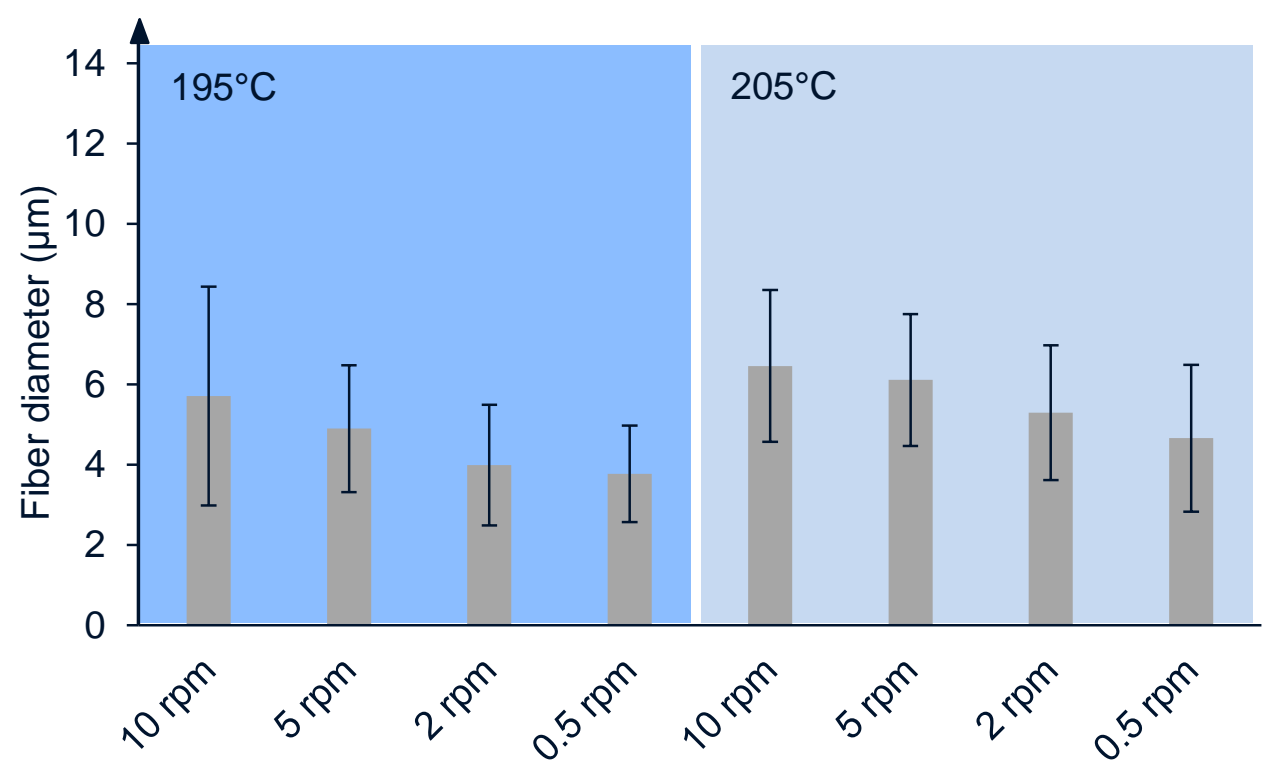

Fig. 4.11: Diameters of PLA fibers containing 6\% (w/w) NaSt produced at $195{ }^{\circ} \mathrm{C}$ with spin pump speeds of $10,5,2$ and $0.5 \mathrm{rpm}$ using the 600 -nozzle meltelectrospinning prototype.

The smallest average fiber diameter $(3.77 \mu \mathrm{m})$ was achieved by processing PLA containing $6 \%(\mathrm{w} / \mathrm{w}) \mathrm{NaSt}$ at $195{ }^{\circ} \mathrm{C}$ with a spin pump speed of $0.5 \mathrm{rpm}$. The smallest single fiber diameter $(1.23 \mu \mathrm{m})$ was achieved with the same additive concentration and temperature but with a spin pump speed of $2 \mathrm{rpm}$. The corresponding images are shown in Figure 4.12.
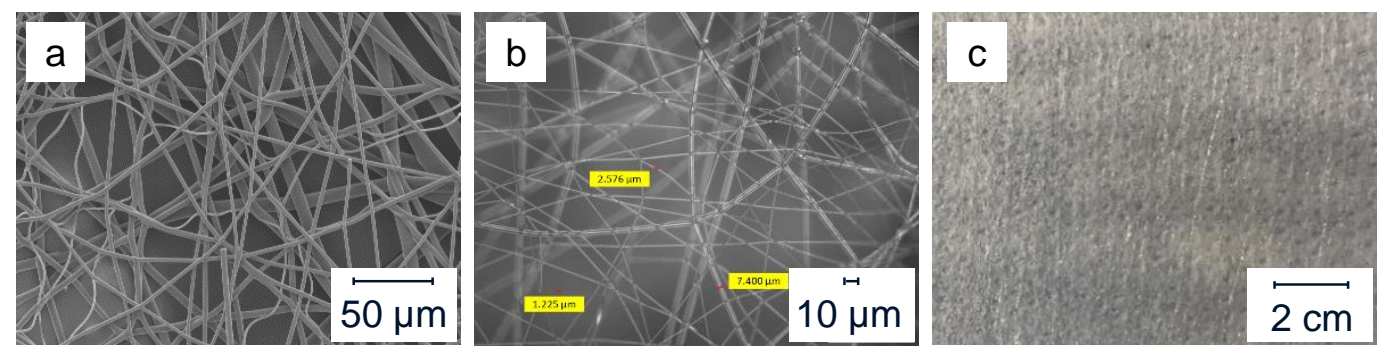

Fig. 4.12: Images of PLA fibers containing 6\% (w/w) NaSt: (a) SEM image $\times 1000$, (b) reflected light microscopy image $\times 200$ indicating the smallest fiber diameter, and (c) unmagnified image showing the appearance of the nonwoven fibers produced using the 600-nozzle melt-electrospinning prototype. 
All measured average fiber diameters and standard deviations are summarized in Table 4.5.

Tab. 4.5: Average fiber diameters and standard deviations produced with the pilotscale melt-electrospinning device at $195^{\circ} \mathrm{C}$ and $205^{\circ} \mathrm{C}$ and reducing spin pump speed from $10 \mathrm{rpm}$ to $0.5 \mathrm{rpm}$.

\begin{tabular}{|c|c|c|c|c|c|}
\hline \multirow{2}{*}{\multicolumn{2}{|c|}{ Material }} & \multicolumn{4}{|c|}{$\begin{array}{c}\text { Fiber diameter }(\mu \mathrm{m}) \\
\text { depending on spin pump speed }\end{array}$} \\
\hline & & 10 rpm & $5 \mathrm{rpm}$ & $2 \mathrm{rpm}$ & $0.5 \mathrm{rpm}$ \\
\hline $\begin{array}{l}\mathrm{PLA}+2 \%(w / w) \\
\mathrm{NaSt}\end{array}$ & \multirow{3}{*}{$\begin{array}{l}\text { O } \\
\stackrel{\circ}{\circ} \\
\text { م }\end{array}$} & $24.97 \pm 4.92$ & $14,66 \pm 3.80$ & - & - \\
\hline $\begin{array}{l}\mathrm{PLA}+4 \%(\mathrm{w} / \mathrm{w}) \\
\mathrm{NaSt}\end{array}$ & & $14.91 \pm 3.35$ & $8.77 \pm 2.13$ & - & - \\
\hline $\begin{array}{l}\mathrm{PLA}+6 \%(\mathrm{w} / \mathrm{w}) \\
\mathrm{NaSt}\end{array}$ & & $5.71 \pm 2.73$ & $4.90 \pm 1.58$ & $3.99 \pm 1.50$ & $3.77 \pm 1.20$ \\
\hline $\begin{array}{l}\mathrm{PLA}+2 \%(\mathrm{w} / \mathrm{w}) \\
\mathrm{NaSt}\end{array}$ & \multirow{3}{*}{$\begin{array}{l}0 \\
\stackrel{\circ}{\circ} \\
\stackrel{N}{N}\end{array}$} & $16.43 \pm 4.83$ & $11.79 \pm 3.59$ & - & - \\
\hline $\begin{array}{l}\mathrm{PLA}+4 \%(\mathrm{w} / \mathrm{w}) \\
\mathrm{NaSt}\end{array}$ & & $8.38 \pm 3.64$ & $6.35 \pm 2.08$ & - & - \\
\hline $\begin{array}{l}\mathrm{PLA}+6 \%(\mathrm{w} / \mathrm{w}) \\
\mathrm{NaSt}\end{array}$ & & $6.46 \pm 1.89$ & $6.11 \pm 1.64$ & $5.30 \pm 1.68$ & $4.66 \pm 1.83$ \\
\hline
\end{tabular}

In order to ensure that the reduction in fiber diameter when reducing the spin pump speed is not associated with the substantial degradation of the PLA due to longer dwell times, GPC measurements were carried out on the nonwovens produced at spin pump speed from $10 \mathrm{rpm}$ to $0.5 \mathrm{rpm}$. No significant reduction in molecular weight could be detected (Appendix: Fig. A4).

To support our observations using the pilot-scale melt-electrospinning device, we have worked out the relationship between additive concentration, temperature, spin pump speed and the resulting average fiber diameter using a Minitab 19 design of experiments (DOE) evaluation. The one-dimensional relationships are shown in Figure 4.13. As described above, the fiber diameter decreases with a reduction in spin pump speed, an increase in temperature, or an increase in additive concentration. The visibility of this trend declines at the highest additive concentration, but is still discernible (Figure 4.11). The effect of uncontrolled environmental factors should also be considered, particularly for the minimal deviations between the fiber diameters at higher additive concentrations. 


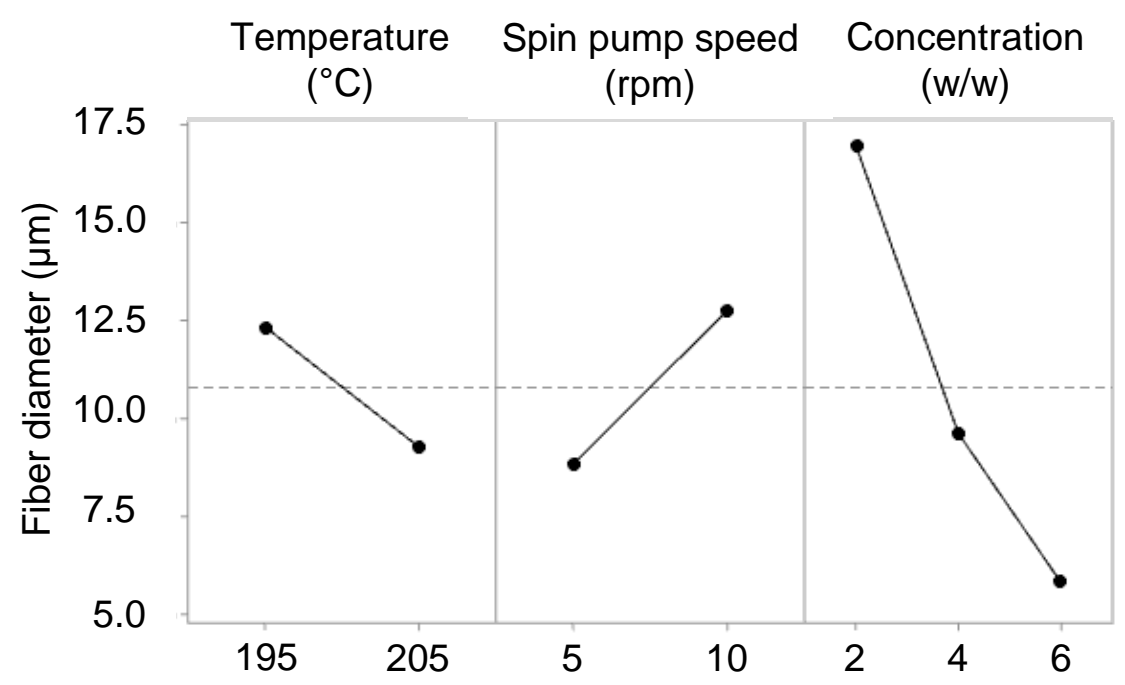

Fig. 4.13: One-dimensional DOE evaluation of the effects of additive concentration, temperature and spin pump speed on the average fiber diameter produced with the pilot-scale device.

Figure 4.14 shows the surface plots of fiber diameter versus (a) additive concentration (NaSt) and spin pump speed, (b) temperature and spin pump speed, (c) additive concentration (NaSt) and temperature. If the temperature remains the same, the increase in additive concentration has a greater effect than the reduction in spinning pump end speed (Figure 4.14a). With constant additive concentration, the temperature influence is higher than the reduction of the spinning pump speed (Figure 4.14b). Furthermore, it can be shown that the temperature influence on the fiber diameter decreases with increasing additive concentration and constant spin pumps speed (Figure 4.14c).

Additionally, a two-way analysis of variance (ANOVA) based on the data set of Figure 4.10, 4.11 and 4.14 was conducted to proof statistical relevance of the observed phenomena using IBM SPSS statistics software. The effect of the independent factors spin pump speed, additive concentration and temperature on the fiber diameter was examined. The Test of Between-Subjects Effects revealed a main effect for the factor additive concentration, $F(2,784)=767.32, p<.001, \eta^{2}=.66$, a main effect for the factor spin pump speed, $F(3,784)=99.57, p<.001, \eta^{2}=.28$, and a main effect for the factor temperature, $F(1,784)=128.70, p<.001, \eta^{2}=.14$. There was a statistically significant interaction between the effects of additive concentration and spin pump speed, $F(2,784)=78.39, \quad p<.001, \quad \eta^{2}=.17, \quad$ spin pump speed and temperature, $F(2,784)=75.52, p<.001, \eta^{2}=.16$, as well as temperature and spin pump speed, $F(3,784)=19.97, p<.001, \eta^{2}=.07$. Comparing the effect strength $\eta$, the independent factor additive concentration has the greatest influence on the fiber diameter. The ANOVA analysis does not consider the effect of material degradation over time; this should be considered in future studies. 
nonwoven (Figure 4.12c). To overcome this phenomenon and improve the overlap, the distance between the pins can be reduced in future studies.

\section{Conclusion and further perspectives}

Using our new pilot-scale melt-electrospinning device, we have produced the first PLAbased fibers in the low micrometer range. The addition of $6 \%(\mathrm{w} / \mathrm{w}) \mathrm{NaSt}$ was required to produce the smallest average fiber diameter $(3.77 \mu \mathrm{m})$ using a spinneret temperature of $195^{\circ} \mathrm{C}$ and a spin pump speed of $0.5 \mathrm{rpm}\left(0.16 \mathrm{~cm}^{3}\right)$. The smallest single fiber diameter $(1.23 \mu \mathrm{m})$ was achieved at the same conditions but using a spin pump speed of $2 \mathrm{rpm}$. A higher quantity of additive promoted material degradation due to the processing and dwell time, which inhibited fiber formation. Fibers with diameters in the low micrometer range were achieved, comparable with previous reports, without the integration of a heated spinning chamber or additional air flow, which are already widely used at the laboratory scale to achieve further stretching of the fibers. The 600-nozzle melt-electrospinning device thus offers significant potential for the further development of thermoplastic, biobased fibers in the sub-micrometer range. The effects of the additive on envisaged nonwoven products and the applicability for biomedical purposes such as scaffolds still needs to be investigated.

The carried out ANOVA revealed statistical significance for all examined, independent factors (spin pump speed, additive concentration and temperature). Evaluated by the effect strength $\eta$, the additive concentration has the greatest influence on the fiber diameter.

At the laboratory scale, we determined the effect of viscosity-reducing additives (NaSt and PES) as well as $\mathrm{NaCl}$, which increases the conductivity of the polymer melt. The smallest average fiber diameter $(16.44 \mu \mathrm{m})$ was achieved by adding $2 \%(\mathrm{w} / \mathrm{w}) \mathrm{NaCl}$ but the spinning process could not be stabilized. The formation of a Taylor cone followed by fiber deposition was achieved with compounds containing NaSt or PES, but the latter did not reduce the viscosity of the melt at the concentrations we tested and its influence on fiber diameter therefore could not be determined. We conclude that the additive NaSt has the greatest potential to optimize the material properties of PLA for melt electrospinning and that further improvements to the process could reduce the minimum fiber diameter even further. In future attempts a combination of the two salts, $\mathrm{NaCl}$ and $\mathrm{NaSt}$, could also be tested in order to combine the respective positive effects of increasing conductivity and lowering viscosity. 


\section{Acknowledgements}

The authors acknowledge support from the Maastricht MultiModal Molecular Imaging Institute, M4I Division of Nanoscopy, Maastricht University, The Netherlands; the Microscopy Department, Institute for Textile Technology, RWTH Aachen University, Germany; Fourné Maschinenbau GmbH, Alfter-Impekoven, Germany; Pötter-Klima Gesellschaft für Nanoheiztechnik mbH, Georgsmarienhütte, Germany; and Schnick Systemtechnik GmbH \& Co. KG, Heiligenhaus, Germany. We would also like to thank Tibor Duris Textilpflege PRELUNA, Ludwigshafen, Germany, for providing the polyesterbased plasticizing agent, Konrad Beukenberg for the preparation of illustrations, and IT consultant Maureen Koenig for her support in the statistical analysis. 


\section{References}

[1] Ma B, Xie J, Jiang J. Rational design of nanofiber scaffolds for orthopedic tissue repair and regeneration. Nanomedicine (Lond). 2013; 8.

[2] Shin S-H, Purevdorj O, Castano O. A short review: recent advances in electrospinning for bone tissue regeneration. J Tissue Eng. 2012; 3.

[3] McClellan P, Landis WJ. Recent applications of coaxial and emulsion electrospinning methods in the field of tissue engineering. Biores Open Access. 2016; 5.

[4] Sill T, Recum HA. Electrospinning: applications in drug delivery and tissue engineering. Biomaterials. 2008; 29.

[5] Khajavi R, Abbasipour M, Bahador A. Electrospun biodegradable nanofibers scaffolds for bone tissue engineering. J Appl Polym Sci. 2016; 133.

[6] Wang J, Li Y, Sun X. Challenges and opportunities of nanostructured materials for aprotic rechargeable lithium-air batteries. Nano Energy. 2013; 2.

[7] Wang X, Drew C, Lee S-H. Electrospun Nanofibrous membranes for highly sensitive optical sensors. Nano Lett. 2002; 2.

[8] Sundarrajan S, Tan KL, Lim SH. Electrospun nanofibers for air filtration applications. Procedia Eng. 2014; 75.

[9] Scholten E, Bromberg L, Rutledge GC. Electrospun polyurethane fibers for absorption of volatile organic compounds from air. ACS Appl Mater Interfaces. $2011 ; 3$.

[10] Bhat GS. Advances in polymeric nanofiber manufacturing technologies. J Nanomater Mol Nanotechnol. 2016; 5.

[11] Bhardwaj N, Kundu SC. Electrospinning: a fascinating fiber fabrication technique. Biotechnol Adv. 2010; 28.

[12] Brown T, Dalton P, Hutmacher DW. Melt electrospinning today: an opportune time for an emerging polymer process. Elsevier. 2016; 56.

[13] Huang Z-M, Zhang YZ, Kotaki M, Ramakrishna S. A review on polymer nanofibers by electrospinning and their applications in nanocomposites. Composites Science and Technology. 2003; 63(15):2223-53.

[14] Greiner A, Wendorff JH. Electrospinning: A Fascinating Method for the Preparation of Ultrathin Fibers. Angewandte Chemie International Edition. 2007;46(30):5670703.

[15] Long Y-Z, Li M-M, Gu C, Wan M, Duvail J-L, Liu Z, et al. Recent advances in synthesis, physical properties and applications of conducting polymer nanotubes and nanofibers. Progress in Polymer Science. 2011; 36(10):1415-42. 
[16] Qin Y, Cheng L, Zhang Y, Chen X, Wang X, He X, et al. Efficient preparation of poly(lactic acid) nanofibers by melt differential electrospinning with addition of acetyl tributyl citrate. Journal of Applied Polymer Science. 2018; 135(31):46554.

[17] Ellringmann T, Wilms C, Warnecke M, Seide G, Gries T. Carbon fiber production costing: a modular approach. Textile Research Journal. 2015; 86(2):178-90.

[18] Dalton PD, Grafahrend D, Klinkhammer K, Klee D, Moller M. Electrospinning of polymer melts: Phenomenological observations. Polymer. 2007; 48(23):6823-33.

[19] Dalton PD, Klinkhammer K, Salber J, Klee D, Möller M. Direct in Vitro Electrospinning with Polymer Melts. Biomacromolecules. 2006; 7(3):686-90.

[20] Zhou H, Green TB, Joo YL. The thermal effects on electrospinning of polylactic acid melts. Polymer (Guildf). 2006; 47.

[21] Nayak R. Polypropylene nanofibers: melt electrospinning versus Meltblowing. Engineering Material: Springer International Publishing; 2017.

[22] Bubakir M, Barhoum A, Li H, Yang W. Handbook of nanofibers (1st edtn), springer nature2017.

[23] $\mathrm{Li} \mathrm{H}$, Chen $\mathrm{H}$, Zhong $\mathrm{X}$. Interjet distance in needleless melt differential electrospinning with umbellate nozzles. J Appl Polym Sci. 2014; 131.

[24] Liu Y, Zhao F, Zhang C, Zhang J, Yang W. Solvent-free preparation of poly(lactic acid) fibers by melt electrospinning using an umbrella-like spray head and alleviation of the problematic thermal degradation. J Serb Chem Soc. 2012; 77.

[25] Hacker C, Seide G, Gries T, Thomas H, Moeller M, editors. Electrospinning of polymer melt: steps toward an upscaled multi-jet process. International Conference latest advances in high tech textiles and textile-based materials; 2009.

[26] Koenig K, Daenicke J, Langensiepen F, Seide G, Schubert DW. From lab to pilot scale: melt electrospun nanofibers of polypropylene with conductive additives. J Nanomater Mol Nanotechnol. 2019; 8(2).

[27] Koenig K, Beukenberg K, Langensiepen F, Seide G. A new prototype meltelectrospinning device for the production of biobased thermoplastic submicrofibers and nanofibers. Biomaterials Research. 2019; 23(1):10.

[28] Zhao F, Liu Y, Yuan H. Orthogonal design study on factors affecting the degradation of polylactic acid fibers of melt electrospinning. J Appl Polym Sci. 2012; 125.

[29] Li X, Liu H, Liu J. Preparation and experimental parameters analysis of laser melt electrospun poly(L-lactide) fibers via orthogonal design. Polym Eng Sci. 2012; 52.

[30] Larrondo L, Manley R SJ. Electrostatic fiber spinning from polymer melts. I. Experimental observations on fiber formation and properties. . J Polym Sci Polym Phys Ed.19:909-20. 
[31] Dalton PD, Lleixà Calvet J, Mourran A. Melt electrospinning of poly-(ethylene glycol-block-e-caprolactone). Biotechnol J. 2006;1.

[32] Yoon YI, Park KE, Lee SJ, Park WH. Fabrication of Microfibrous and Nano/Microfibrous Scaffolds: Melt and Hybrid Electrospinning and Surface Modification of Poly(L-lactic acid) with Plasticizer. BioMed Research International. 2013:10.

[33] Brown TD, Dalton PD, Hutmacher DW. Direct writing by way of melt electrospinning. Adv Mater. 2011; 23.

[34] Lyons J, Li C, Ko F. Melt-electrospinning part I: processing parameters and geometric properties. Polymer. 2004; 45(22):7597-603.

[35] Nayak R. Effect of viscosity and electrical conductivity on the morphology and fiber diameter in melt electrospinning of polypropylene. Textile Research Journal. 2013; 83(6):606.

[36] Yin H-M, Qian J, Zhang J, et al. Engineering porous poly(lactic acid) scaffolds with high mechanical performance via a solid state extrusion/porogen leaching approach. Polymers 2016; 8: 213.

[37] Lin HR, Kuo CJ, Yang CY, et al. Preparation of macroporous biodegradable PLGA scaffolds for cell attachment with the use of mixed salts as porogen additives. J Biomed Mater Res 2002; 63: 271-279.

[38] Zhmayev E, Cho D and Joo YL. Nanofibers from gasassisted polymer melt electrospinning. Polymer 2010; 51: 4140-4144.

[39] Ogata N, Yamaguchi S, Shimada N, et al. Poly(lactide) nanofibers produced by a melt-electrospinning system with a laser melting device. J Appl Polym Sci 2007; 104: 1640-1645.

[40] Carroll CP, Zhmayev E, Kalra V, et al. Nanofibers from electrically driven viscoelastic jets: modeling and experiments. Korea-Aust Rheol J 2008; 20: 153164.

[41] Chrzanowska O, Struszczyk M and Krucin' ska I. Small diameter tubular structure design using solvent-free textile techniques. J Appl Polym Sci 2014; 131.

[42] Mazalevska O, Struszczyk M, Chrzanowski M, et al. Application of electrospinning for vascular prothesis design-preliminary results. Fibres Text East Eur 2011; 19: 46-52.

[43] Mazalevska O, Struszczyk MH and Krucinska I. Design of vascular prostheses by melt electrospinning-structural characterizations. J Appl Polym Sci 2013; 129: 779-792.

[44] Zhmayev E, Zhou H and Joo YL. Modeling of non-isothermal polymer jets in melt electrospinning. J NonNewton Fluid 2008; 153: 95-108. 
[45] NatureWorks. Ingeo Biopolymer 6201D Technical Data Sheet, https://www.natureworksllc.com/media/Files/NatureWorks/TechnicalDocuments/Technical-DataSheets/TechnicalDataSheet_6201D_fiber-meltspinning_pdf (accessed 7 May 2019).

[46] Hacker C. Anlagenentwicklung für das Elektroschmelzspinnen von Feinfaservliesstoffen für die Abwasseraufbereitung. PhD Thesis, RWTH Aachen University, Germany, 2015.

[47] Hacker C, Fourne R, Rübsam U, et al. Challenges of the meltelectrospinning process: an economical and technical window of opportunity [Herausforderungen des Schmelzelektrospinns: wirtschaftliche und technische Potentiale und Möglichkeiten]. Austria: Österreichisches Chemiefaser-Institut, 2013.

[48] Lin T. The charge effect of cationic surfactants on the elimination of fibre beads in the electrospinning of polystyrene. Nanotechnology 2004; 15: 1375.

[49] Nayak R, Kyratzis IL, Truong YB, et al. Meltelectrospinning of polypropylene with conductive additives. J Mat Sci 2012; 47: 6387-6396.

[50] Saeidlou S. Poly (lactic acid) crystallization. Prog Polym Sci 2012; 37: 1657. 



\section{Chapter 4}

\section{Polylactic acid in melt electrospinning}

Biobased dyes as conductive additives to reduce the diameter of polylactic acid fibers during melt electrospinning

K. Koenig ${ }^{\star}$, N. Balakrishnan*, S. Hermanns, F. Langensiepen, G. Seide *both authors contributed equally

Materials. 2020; 13(5):1055.

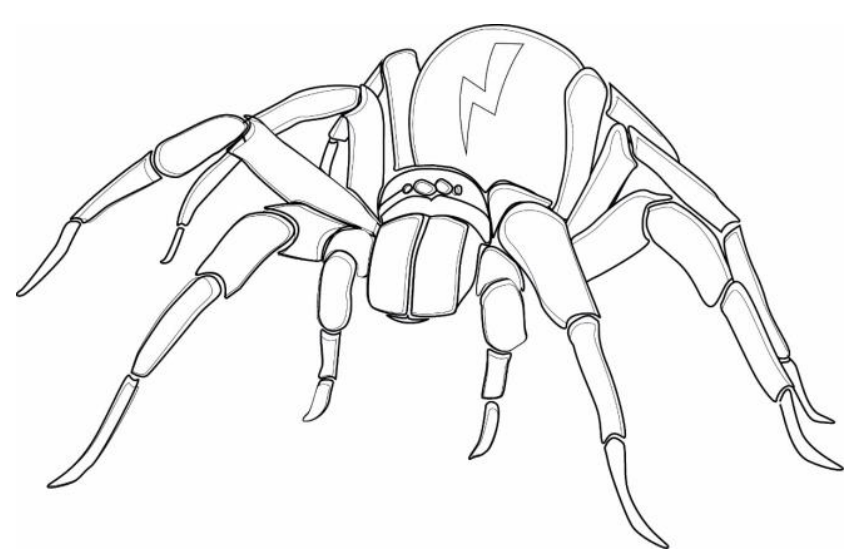




\title{
Biobased dyes as conductive additives to reduce the diameter of polylactic acid fibers during melt electrospinning
}

\begin{abstract}
Electrospinning is widely used for the manufacture of fibers in the low micrometer to nanometer range, allowing the fabrication of flexible materials with a high surface area. A distinction is made between solution and melt electrospinning. The former produces thinner fibers but requires hazardous solvents, whereas the latter is more environmentally sustainable because solvents are not required. However, the viscous melt requires high process temperatures and its low conductivity leads to thicker fibers. Here we describe the first use of the biobased dyes alizarin, hematoxylin and quercetin as conductive additives to reduce the diameter of polylactic acid (PLA) fibers produced by melt electrospinning, combined with a biobased plasticizer to reduce the melt viscosity. The formation of a Taylor cone followed by continuous fiber deposition was observed for all PLA compounds, reducing the fiber diameter by up to $77 \%$ compared to pure PLA. The smallest average fiber diameter of $16.04 \mu \mathrm{m}$ was achieved by adding $2 \%(w / w)$ hematoxylin. Comparative analysis revealed that the melt-electrospun fibers had a low degree of crystallinity compared to drawn filament controls, resembling partially-oriented filaments. Our results form the basis of an economical and environmentally friendly process that could ultimately provide an alternative to industrial solution electrospinning.
\end{abstract}

\section{Introduction}

Electrospinning is a simple, versatile and cost-effective method to produce fibers in the low micrometer to nanometer range, thus making a significant contribution to the booming nanotechnology industry [1-3]. The beneficial properties of such fibers include their flexibility and enormous surface area, leading to applications in medicine [4-8], filtration and separation [9, 10], electronics and energy [11, 12], and textile manufacturing [13-15].

Electrospinning involves the exposure of liquids to strong electric fields. When a large potential difference (tens of kilovolts) is applied to a liquid flowing through a capillary, the liquid forms a jet that may undergo whip-like movements, stretching the fluid and yielding microscale or nanoscale fibers that are deposited on a collector [15]. The two principal types of electrospinning are solution electrospinning, where the polymer is dissolved in a solvent that evaporates to produce the fibers, and melt electrospinning, where a molten polymer is cooled to produce the fibers [1]. Solution electrospinning is easier to implement, has been studied more widely, and is favored by industry, despite the 
environmental hazards posed when toxic solvents are required. In contrast, research and technology uptake in the field of melt electrospinning has been held back by complex equipment requirements [16], the problem of electric discharge [17], and the high temperature, high viscosity and low conductivity of the polymer melt [18]. Accordingly, parameters that affect the viscosity, conductivity, thermal and structural properties of solution-electrospun fibers are well understood, allowing the production of finer fibers, whereas equivalent studies focusing on melt electrospinning are still at an early stage $[1,19]$.

The wider adoption of melt electrospinning could help to reduce the environmental footprint of current industrial electrospinning processes, which require an expensive solvent recovery process and present a high risk of toxic solvent carryover into the final product. For example, one of the commonly used materials in industrial electrospinning process is polylactic acid (PLA) because it is a sustainable polymer made from renewable agricultural resources and is reported to be industrial compostable. Commercial low molecular weight PLA was reported to have a biodegradation degree of $72 \%$ after 110 days under aerobic conditions [20]. However, PLA sub-microfibers are usually prepared using the toxic solvents dichloromethane, chloroform or $\mathrm{N}, \mathrm{N}$-dimethylformamide [21]. It is therefore desirable to improve melt-electrospinning technology, aiming to reduce the fiber diameter by overcoming the limitations described above. One promising approach is the use of additives to increase the conductivity of polymer melts, and plasticizers to reduce their viscosity, in order to produce thinner fibers [22-24]. PLA is the most commercially available biobased and biodegradable thermoplastic polymer with increasing use in the textile sector to replace petroleum-based polymers [25]. It is therefore an important substrate for melt-electrospinning technology. PLA fibers produced by melt electrospinning have been modified by adding a plasticizer [21, 26], and by adjusting the device during fabrication to facilitate airflow [27] or incorporate laser heating [17]. These approaches led to the production of fibers with diameters in the range $0.2-50 \mu \mathrm{m}[17,18,21,27-33]$. However, most of the additives used thus far are unsustainable chemicals that offset the environmental advantages of biobased polymers. They are difficult to disperse in the polymer melt, or they adsorb water and therefore interfere with high-temperature melt-electrospinning processes [21, 26, 34].

Sustainable colorants are used extensively in the textile industry, offering a promising alternative to conventional additives [35]. Colorants can be classified as dyes or pigments. Dyes are molecules that can be solubilized in a polymer substrate, they have good chemical affinity for the polymer, and therefore retain transparency; in contrast, pigments are insoluble in the polymer substrate and are dispersed as very fine particles (Figure 4.15). 

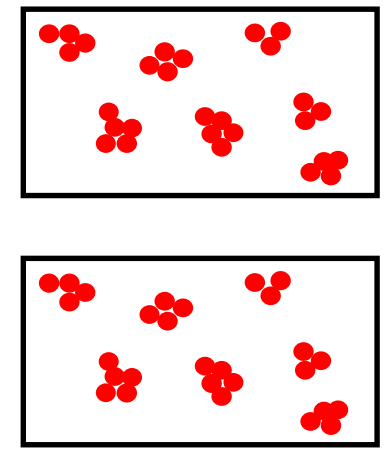

Dye solubilization
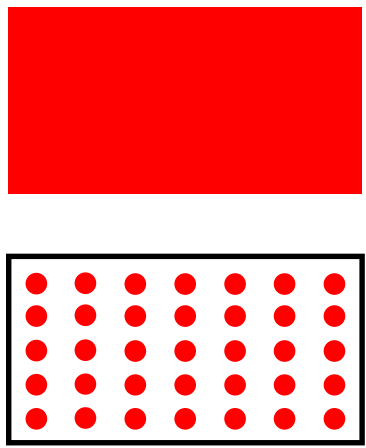

Fig. 4.15: Schematic representation of dye solubilization and pigment dispersion.

Common organic textile dyes include alizarin and curcuma, whereas common pigments include copper phthalocyanine and carbon black [36]. Colorants such as alizarin, purpurin and phthalocyanine have already been used to manufacture electronics such as field effect transistors and dye-sensitized solar cells [37] because the presence of functional groups and/or $\pi$-conjugation improves conductivity, and charge carrier mobility may exceed $1 \mathrm{~cm}^{2} / \mathrm{Vs}$ [38].

The aim of this study was to investigate the ability of three biobased dyes to increase the conductivity of molten PLA during melt electrospinning, combined with a biobased plasticizer to reduce melt viscosity for an overall reduction of the obtained fiber diameters. We selected the biobased dyes alizarin, quercetin and hematoxylin, which have not previously been used in a melt-electrospinning process, and determined their effect on the diameter of PLA fibers manufactured using a single-nozzle meltelectrospinning device. We compared fibers incorporating different weight percentages of dye with or without the biobased plasticizer. We determined the influence of the additives on viscosity, conductivity, degradation and thermal behavior. We also compared the morphology and crystallization behavior of melt-electrospun fibers to meltspun fibers with different draw ratios. Our results can be used to develop an environmentally beneficial melt-electrospinning process for the manufacture of microscale and nanoscale fibers.

\section{Experimental}

\section{Materials}

PLA grade L130 (Total|Corbion, Gorinchem, Netherlands) was used as the base polymer for all experiments. The following specifications were reported by the manufacturer: Lcontent $\geq 99 \%$, glass transition temperature $\left(T_{g}\right) \sim 60^{\circ} \mathrm{C}$, and melt flow index $=24 \mathrm{~g} / 10 \mathrm{~min}$ at $210^{\circ} \mathrm{C} / 2.16 \mathrm{~kg}$. The chemical structures and melting points of the biobased dyes alizarin, quercetin, and hematoxylin (Sigma-Aldrich, Zwijndrecht, Netherlands) are presented in Table 4.6. 
Tab. 4.6: Chemical structures and melting points of the dyes alizarin, hematoxylin and quercetin.

Dye

Liquid dyes (Rowasol, Pinneberg, Germany) were prepared by stirring 25\% (w/w) of the biobased dyes with a plasticizer based on vegetable oil. The dyes were used without plasticizer to test their effect on melt conductivity and with plasticizer to determine the combined effect of increasing the conductivity and reducing the viscosity of the melt.

\section{Micro-compounder}

PLA was vacuum dried at $80^{\circ} \mathrm{C}$ overnight before compounding. PLA compounds were made by mixing with $1 \%$ or $2 \%(\mathrm{w} / \mathrm{w})$ of each additive in a micro-compounder (Xplore, 
Sittard, Netherlands) at $200{ }^{\circ} \mathrm{C}$ with a screw speed of $100 \mathrm{rpm}$ for $2 \mathrm{~min}$. The list of compounds and their abbreviations are presented in Table 4.7.

Tab. 4.7: Compound abbreviations according to the dye and weight percent.

\begin{tabular}{|l|l|l|l|}
\hline $\begin{array}{l}\text { Compound } \\
\text { abbreviation }\end{array}$ & Dye & $\%(\mathbf{w} / \mathbf{w})$ of dye & $\%(\mathbf{w} / \mathbf{w})$ of plasticizer \\
\hline A1 & Alizarin & 1 & 0 \\
\hline A2 & Alizarin & 2 & 0 \\
\hline LA1 & Liquid Alizarin & 0.25 & 0.75 \\
\hline LA2 & Liquid Alizarin & 0.5 & 1.5 \\
\hline H1 & Hematoxylin & 1 & 0 \\
\hline H2 & Hematoxylin & 2 & 0 \\
\hline LH1 & Liquid Hematoxylin & 0.25 & 0.75 \\
\hline LH2 & Liquid Hematoxylin & 0.5 & 1.5 \\
\hline Q1 & Quercetin & 1 & 0 \\
\hline Q2 & Quercetin & 2 & 0 \\
\hline LQ1 & Liquid Quercetin & 0.25 & 0.75 \\
\hline LQ2 & Liquid Quercetin & 0.5 & 1.5 \\
\hline
\end{tabular}

In the subsequent evaluation and discussion, it must be taken into account that a direct comparison of results is only feasible between the individual dye compounds or liquid dye compounds due to the changed dye concentration of the liquid dyes.

\section{Melt-spinning equipment}

Partially-oriented filaments (PFs) were prepared using a KETSE 20/40 twin-screw extruder (Brabender, Duisburg, Germany) with a spinning head. Melt spinning was performed at $200^{\circ} \mathrm{C}$ using a spinneret with 24 holes, each $0.4 \mathrm{~mm}$ in diameter, and a length to diameter ratio of two. A constant throughput was maintained and the multifilaments were wound at $150 \mathrm{~m} / \mathrm{min}$. The PFs were post-drawn at $100^{\circ} \mathrm{C}$ with a draw ratio of 5 to obtain drawn filaments (DFs). The crystallinity of these filaments was compared to the melt-electrospun fibers.

\section{Melt-electrospinning equipment}

We used a self-configured laboratory-scale single-fiber melt-electrospinning device consisting of five major components: temperature controller, high-voltage power supply, heating elements, syringe pump, and collector (Figure 4.16). The device was equipped with JCS-33A temperature process controllers (Shinko Technos, Osaka, Japan) and PT 
100 platinum thermocouples (Omega Engineering, Deckenpfron, Germany) to control the melting temperature. The temperature was set to $275^{\circ} \mathrm{C}$ for the pure polymer and polymer with additives. A KNH65 high-voltage generator (Eltex-Elektrostatik, Weil am Rhein, Germany) with a voltage range of $6-60 \mathrm{kV}$ was used. During the meltelectrospinning experiments, the voltage was kept constant at $50 \mathrm{kV}$. A positive voltage was applied to the collector while grounding the spinneret. A flat aluminum plate $(6 \mathrm{~cm})$ overlaid with a thin paperboard was used as a collector. The distance between the spinneret and collector was set at $10 \mathrm{~cm}$ for all trials. An 11 Plus spin pump (Harvard Apparatus, Cambridge, USA) was used with a constant delivery rate of $4 \mathrm{~mL} / \mathrm{h}$. A 2-mL glass syringe (Poulten \& Graf, Wertheim, Germany) with a nozzle orifice of $1 \mathrm{~mm}$ served as the spinneret.

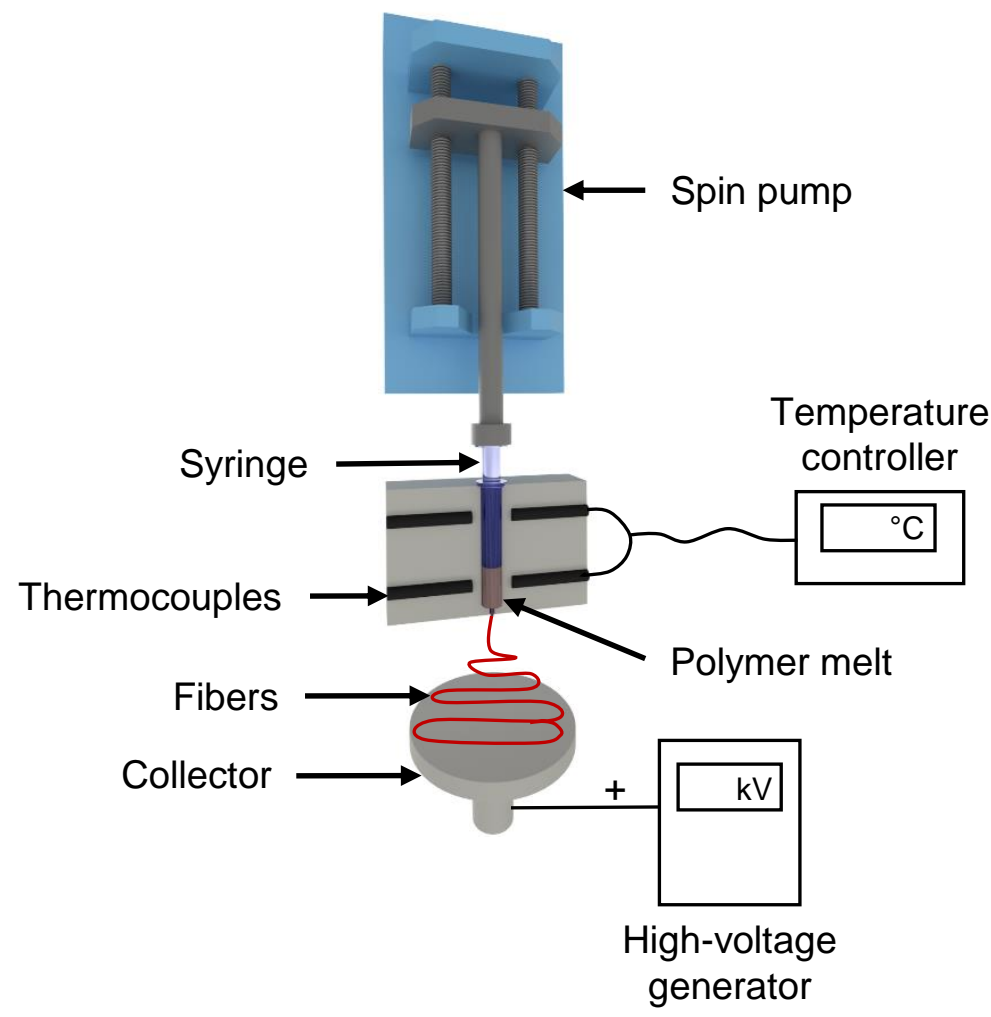

Fig. 4.16: Laboratory single-fiber melt-electrospinning setup.

\section{Characterization of compounds}

Differential scanning calorimetry (DSC) was performed using a Q2000 device (TA Instruments, New Castle, USA) to determine the influence of the additive on the different thermal transition temperatures of PLA. The tests were carried out at a heating rate of $10^{\circ} \mathrm{C} / \mathrm{min}$ between 25 and $200^{\circ} \mathrm{C}$ with a sample size of $\sim 5 \mathrm{mg}$. The parameters were kept constant for all samples to ensure comparability. TA universal analysis software was used to visualize and compare the data. We compared the $T_{g}$, cold crystallization temperature $\left(T_{c c}\right)$, melting point $\left(T_{m}\right)$, and percentage crystallinity $\left(X_{c}\right)$. The melt enthalpy of $100 \%$ crystalline PLA was considered to be $93.7 \mathrm{~J} / \mathrm{g}$ [39]. The $X_{c}$ was determined through the following formula: 


$$
X_{c}=\left(H_{m}-H_{c}\right) \cdot 100 / H_{m 0}
$$

$\mathrm{H}_{\mathrm{m} 0}$ is the melt enthalpy of the material at $100 \%$ crystallinity, $\mathrm{H}_{\mathrm{m}}$ is the melting enthalpy at $T_{m}$ and $H_{c}$ is the enthalpy of cold crystallization at $T_{c c}$. Since the reorganization peak prior to melting peak was caused by rearrangement of imperfect crystals, this was not included in calculating overall crystallinity. All compounds as well as the virgin PLA were processed identically and prepared using the same protocol, so any differences in material properties should primarily reflect the nature and quantity of additives.

A Q5000 device (TA instruments) was used to carry out thermogravimetric analysis (TGA) at a heating rate of $10^{\circ} \mathrm{C} / \mathrm{min}$ under nitrogen flow up to $500^{\circ} \mathrm{C}$. The temperatures at $5 \%$ and $50 \%$ weight loss were determined using TA universal analysis software and the values were compared to determine the influence of additives on the thermal stability of PLA.

Rheological characterization was carried out using a Discovery HR1 hybrid rheometer (TA Instruments). We performed one flow sweep using a $25-\mathrm{mm}$ plate with an increasing shear rate $(0.01-500 \mathrm{rad} / \mathrm{s})$. The gap between the plates was maintained at $1000 \mu \mathrm{m}$, and the strain amplitude and environment temperature were maintained at $0.5 \%$ and $200{ }^{\circ} \mathrm{C}$, respectively. For better comparability, the viscosity of the pure PLA and all the compounds are presented at a shear rate of $5 \mathrm{rad} / \mathrm{s}$.

The TGA experiment was not isothermal and could only determine the weight loss, so we also measured actual degradation in terms of molecular weight after compounding and melt electrospinning by gel permeation chromatography (GPC) using a 1260 Infinity System (Agilent Technologies, Santa Clara, USA). We used hexafluor-2-isopropanol (HFIP) containing $0.19 \%$ sodium trifluoroacetate as the mobile phase at a flow rate of $0.33 \mathrm{~mL} / \mathrm{min}$. Solutions were prepared by dissolving $5 \mathrm{mg}$ of pure PLA and the various compounds in HFIP for $\sim 2 \mathrm{~h}$, passing the solutions through a $0.2-\mu \mathrm{m}$ polyetrafluoroethylene filter, and injecting them into a modified silica column filled with $7-\mu \mathrm{m}$ particles (Polymer Standards Service, Mainz, Germany). The experiment was calibrated against a standard polymethyl methacrylate polymer $\left(1.0 \times 10^{5} \mathrm{~g} / \mathrm{mol}\right)$ and the relative molecular weight $\left(M_{w}\right)$, number average molar mass $\left(M_{n}\right)$, and polydispersity index (PDI) of each polymer were recorded and compared.

The electrical resistance of the pure polymer and compounds was measured at an elevated temperature of $325^{\circ} \mathrm{C}$ using a Keithley 617 electrometer (Tektronix Inc., Beaverton, USA) as shown in Figure 4.17. The experimental setup has been previously used for conductivity measurements of polymer melts [19]. The polymer granulate was melted using band heaters, and two electrodes, $6 \mathrm{~mm}$ apart, were dipped in the melt and connected to the electrometer. The electrical current flowing between the electrodes was measured by applying a constant $10 \mathrm{~V}$. 


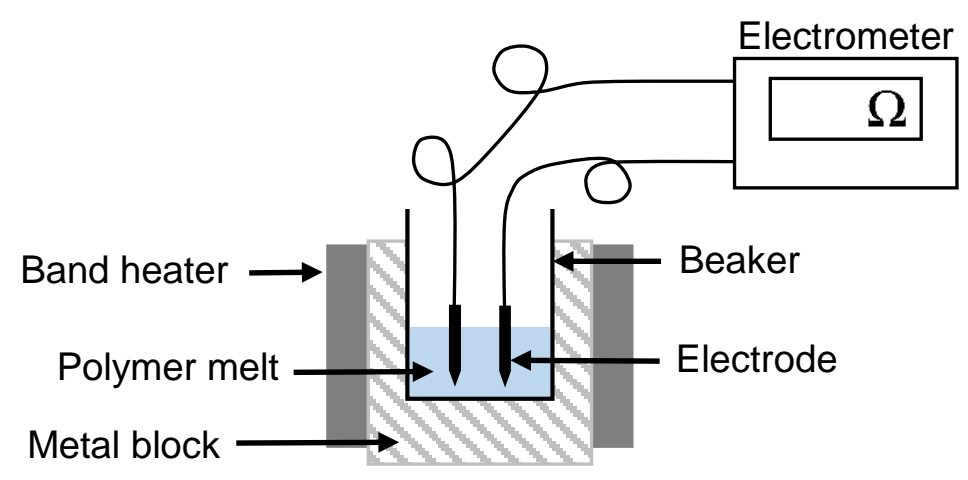

Fig. 4.17: Configuration used for the measurement of electrical resistance.

\section{Characterization of the fibers}

Fiber diameters were determined by reflected light microscopy using a DM4000 M instrument (Leica Microsystems, Wetzlar, Germany) at 100-200× magnification, and images were captured using Leica Application Suite software. Ten images representing different areas of each nonwoven were used to determine the average fiber diameter. DSC was performed on all melt-electrospun fibers to determine the effect of additives and the electrospinning process. DSC was also carried out on the PFs and DFs, as well as the melt-electrospun PLA fibers under the same testing conditions. The thermal transition temperatures and $X_{c}$ values were compared. Polarized optical microscopy (POM) was used to investigate the crystallinity of the melt-electrospun filaments, PFs and DFs. An Olympus BX53 microscope and DP26 camera (Olympus BV, Leiderdorp, Netherlands) were used to capture the images at $50 \times$ magnification. The images were screened for birefringence. The relationship between the electrical resistance, melt viscosity, and average fiber diameter was visualized in surface plots using Minitab19 analysis software.

\section{Cost analysis}

Only $2-10 \%$ of the liquid processed during solution electrospinning is the polymer (the rest is solvent that evaporates) whereas $100 \%$ of the processed liquid solidifies into fibers during melt electrospinning [1]. The cost of PLA is $2-4 € / \mathrm{kg}$ depending on the grade, and we used a nominal value of $2.1 € / \mathrm{kg}$ in our cost model. In solution electrospinning, PLA is prepared as a $10 \%$ solution in chloroform, which costs $\sim 100 € /$ liter. Accordingly, $10 \mathrm{~L}$ of chloroform is required to make $1 \mathrm{~kg}$ of PLA fiber. The total material cost of $1 \mathrm{~kg}$ of PLA fiber is therefore $\sim 1000 €$ for the first production cycle without recovery of the solvent and solvent disposal according to standards. For an off-site solvent recovery the cost is estimated to be $\sim 100 €$ per $200 \mathrm{~L}$ of solvent [40]. Considering the case of production of $1 \mathrm{~kg}$ of PLA fiber, when $90 \%$ of the solvent used can be recovered, $9 \mathrm{~L}$ of the solvent can be reused and it would cost $\sim 4.5 €$. For the remaining $10 \%$, the cost of purchasing new solvent would still be $\sim 100 €$ and the overall solvent cost would be more than $100 €$. In contrast, organic dyes as conductive additives are much less expensive. For example, 
alizarin costs $\sim 900 € / \mathrm{kg}$ and $1 \mathrm{~kg}$ of PLA fiber containing $2 \%(\mathrm{w} / \mathrm{w})$ of this dye would cost $3 €$. Therefore, using organic dyes not only makes the process more sustainable, it is also economical.

\section{Methodology}

Our overall workflow is summarized in Figure 4.18. The PLA was characterized and dried before melt electrospinning, and the diameter of the melt-electrospun pure PLA fiber was measured. We then tested various combinations of additives (dyes with or without plasticizer) to reduce the viscosity of the melt and increase its conductivity, in order to produce thinner fibers. We prepared 12 compounds in total (Table 2) representing each of the three dyes at concentrations of $1 \% \mathrm{w} / \mathrm{w}(\mathrm{A} 1, \mathrm{H} 1$ and $\mathrm{Q} 1)$ and $2 \% \mathrm{w} / \mathrm{w}$ (A2, H2 and $\mathrm{Q} 2)$, as well as the liquid dyes in plasticizer also at additive concentrations of $1 \% \mathrm{w} / \mathrm{w}$ (LA1, LH1 and LQ1) and 2\% w/w (LA2, LH2 and LQ2). The compound names were based on the initial letter of each dye: $A=$ alizarin, $H=$ hematoxylin and $Q=$ quercetin, with $L$ referring to the liquid form. Melt electrospinning was carried out with the compounds and the diameter of the resulting fibers was measured. All other process parameters (e.g., temperature, throughput, electric field strength) were kept constant.

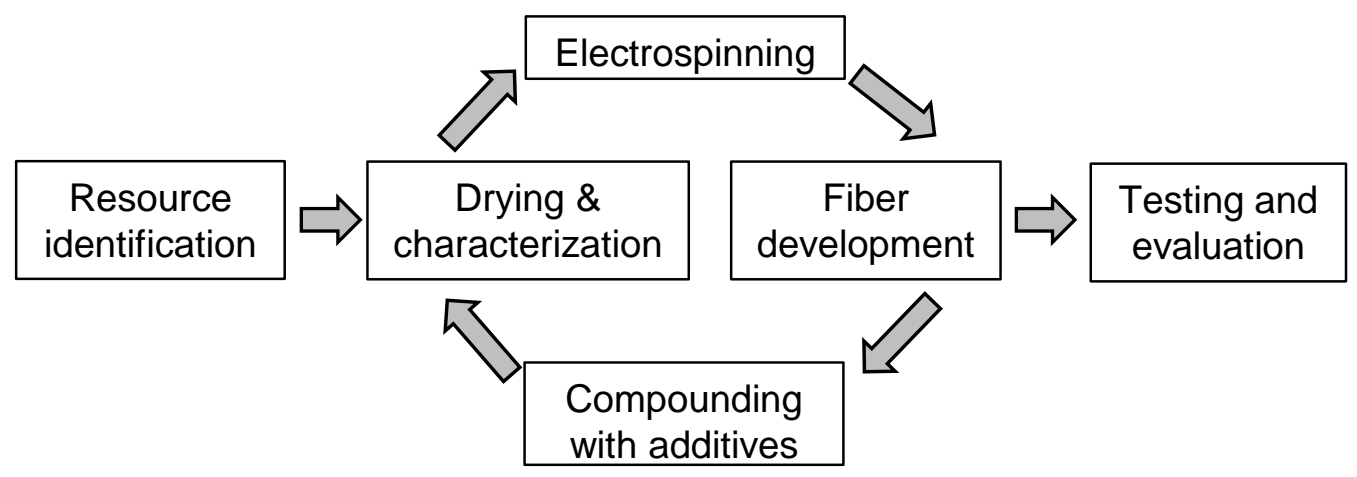

Fig. 4.18: Overview of the experimental workflow.

\section{Results and discussion}

\section{Thermal properties of the PLA compounds}

The DSC thermograms of PLA and A1 are compared in Figure 4.19 as a representative example of the experiments because all the compounds behaved in a similar manner. 


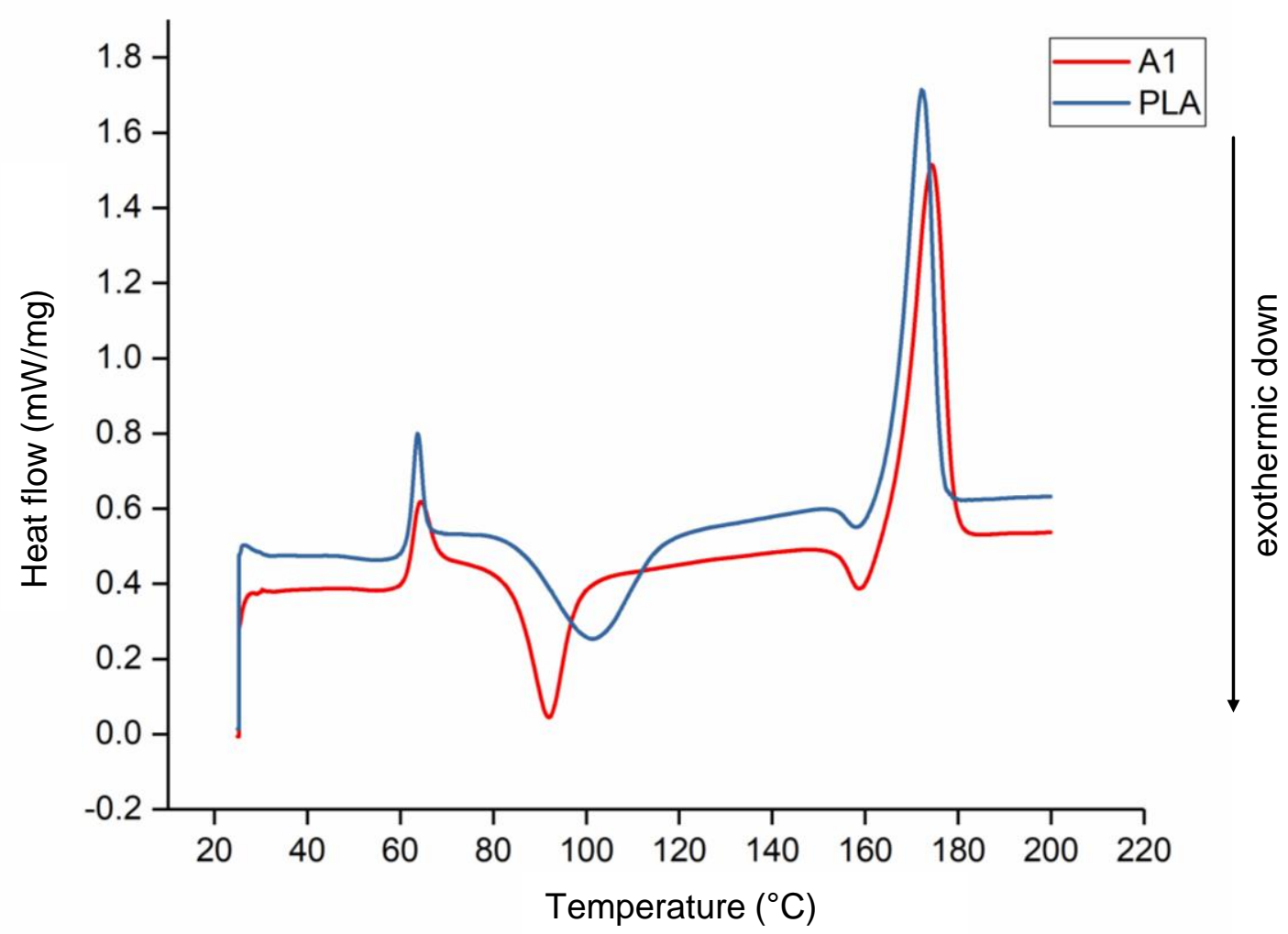

Fig. 4.19: DSC thermogram of PLA and compound A1.

The $T_{g}, T_{c c}, T_{m}$ and $X_{c}$ values of each compound are compared visually in Figures 4.20 and 4.21. The $T_{g}$ and $T_{m}$ did not change significantly and remained at $\sim 60^{\circ} \mathrm{C}$ and $\sim 173^{\circ} \mathrm{C}$, respectively, regardless of the additive and weight percent. The temperature values for PLA are consistent with those previously reported in literature [41, 42]. In contrast, the additives had a significant effect on $\mathrm{T}_{\mathrm{cc}}$. The $\mathrm{T}_{\mathrm{cc}}$ of PLA was $\sim 101^{\circ} \mathrm{C}$ but this declined to $91.30^{\circ} \mathrm{C}$ for $\mathrm{A} 1$ and $87.20^{\circ} \mathrm{C}$ for $\mathrm{A} 2$. Furthermore, the $\mathrm{X}_{\mathrm{c}}$ of PLA was $21.47 \%$, but this increased to $30.72 \%$ for $A 1$ and $29.60 \%$ for $A 2$. The $X_{c}$ of the liquid alizarin compounds also increased compared to pure PLA. Although the $T_{c c}$ of the hematoxylin and liquid hematoxylin compounds was not much lower than the value for pure PLA, the $X_{c}$ of these compounds increased compared to pure PLA. There are two possible explanations for this behavior observed in the cases of these compounds. First, the dye may induce nucleation, as previously reported for polypropylene samples containing colorants $[43,44]$. Second, the dye may trigger the degradation of PLA, leading to shorter and more mobile polymer chains that are more likely to undergo crystallization. This was explored by GPC analysis (Section "Effects of additives on polymer degradation"). The $\mathrm{T}_{\mathrm{cc}}$ of compounds Q1 and Q2 was similar to that of PLA, but the $\mathrm{X}_{c}$ decreased from $21.47 \%$ to $18.47 \%$ and $12.97 \%$, respectively. Also in the case of quercetin, two possible explanations should be considered. The first is that the added quercetin affects the chain mobility and thus disturbs the crystallization process. Similar observations were reported when adding nigrosine dye to polyamide 66 [45]. In case of $L Q$, since the overall content of quercetin is lower, the hindrance to crystallization is also 
lower. The second possible explanation is the degradation theory as explained earlier in the case of hematoxylin. This was also explored by GPC analysis (Section "Effects of additives on polymer degradation").

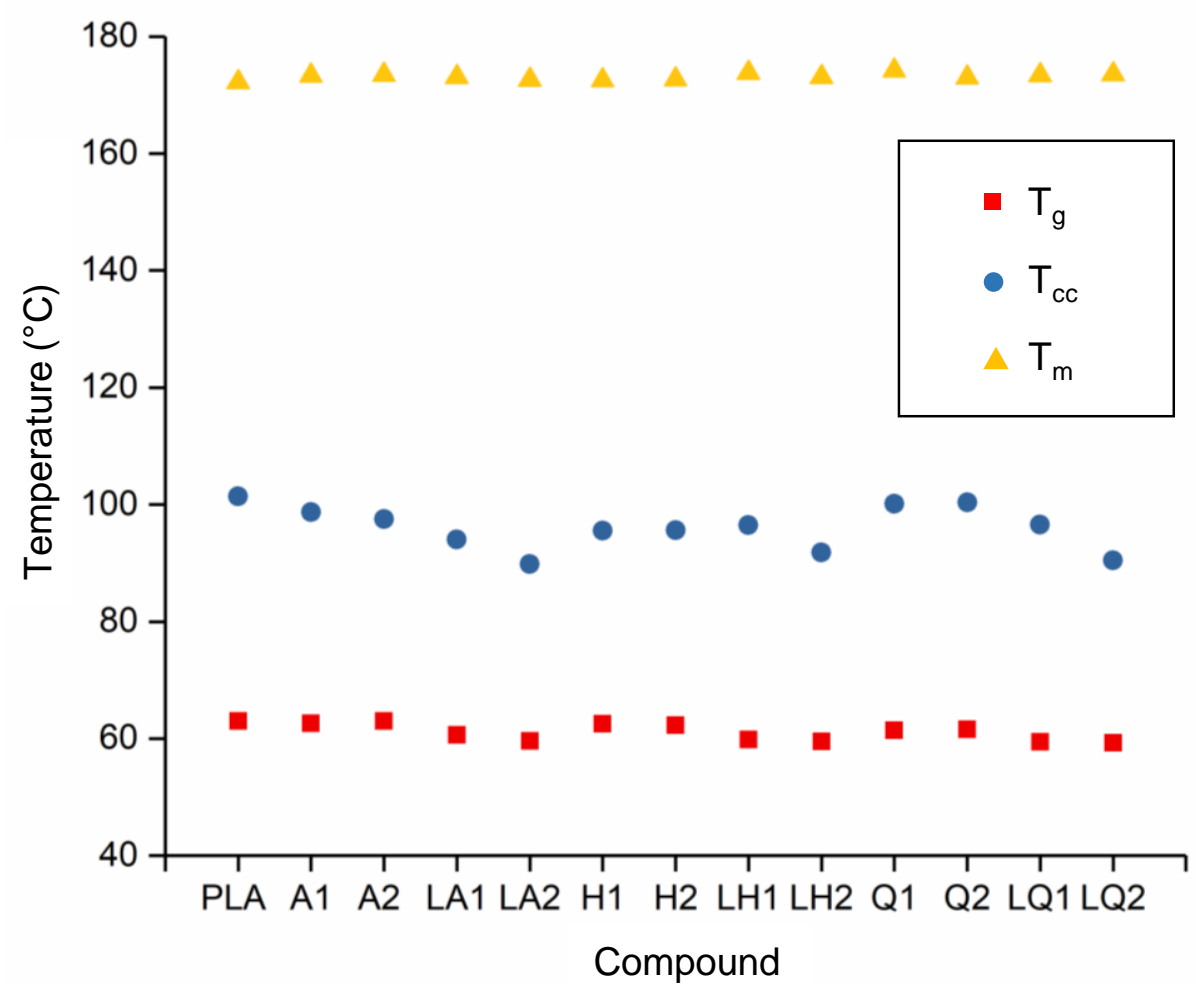

Fig. 4.20: Thermal transition temperature of PLA and the PLA/dye compounds.

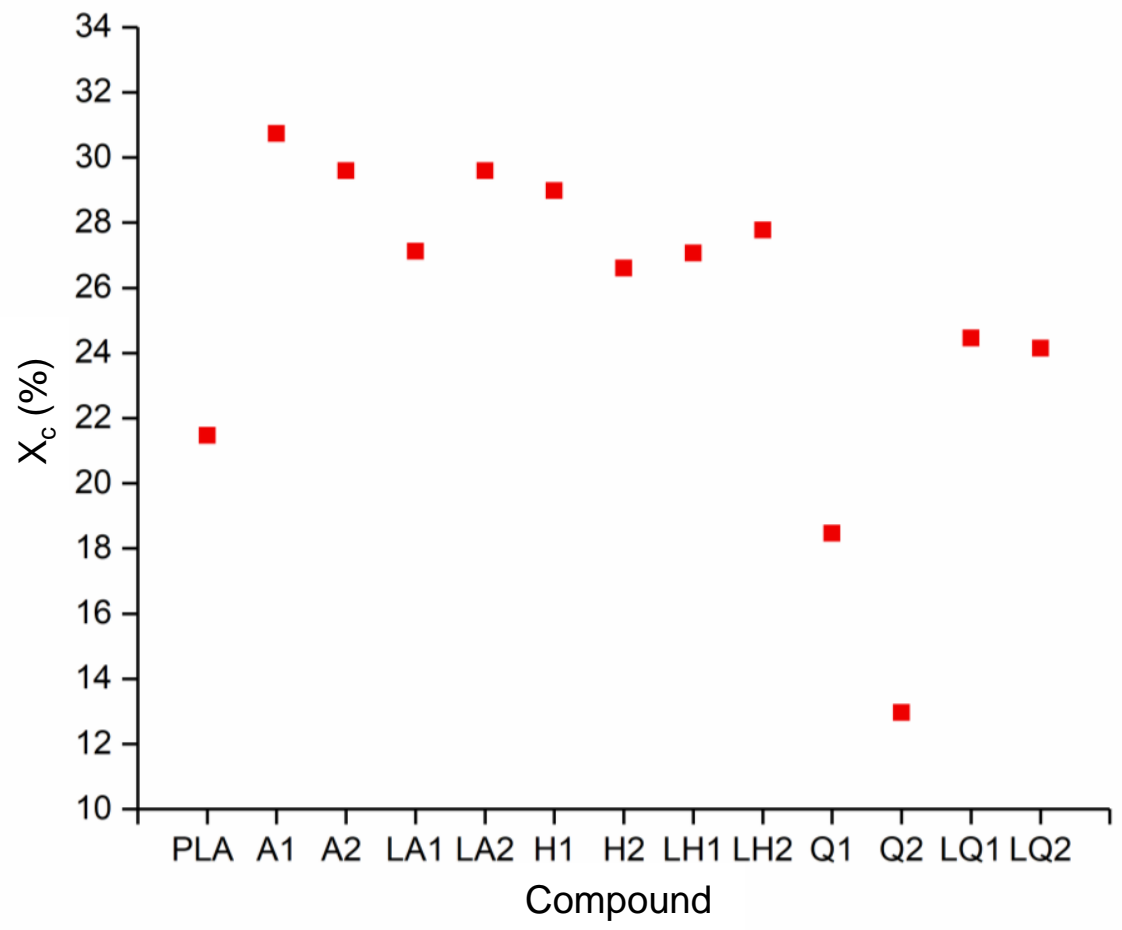

Fig. 4.21: Crystallinity $\left(\mathrm{X}_{c}\right)$ of PLA and the PLA/dye compounds. 
The decomposition temperatures of pure PLA and its compounds were determined by TGA. The temperatures at which $5 \%$ and $50 \%$ weight loss occurred are compared in Figure 4.22.

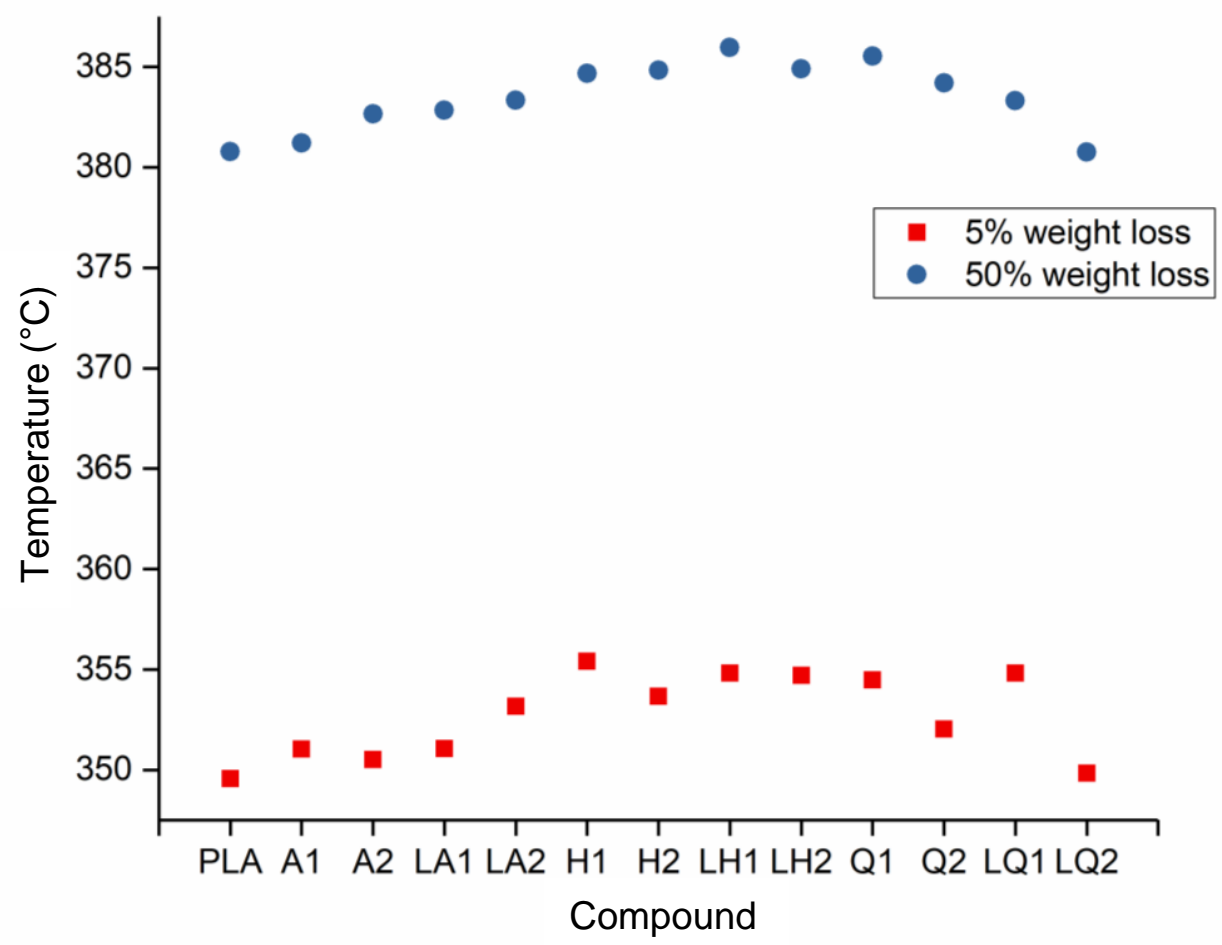

Fig. 4.22: Temperature at $5 \%$ and $50 \%$ weight loss of PLA and the PLA/dye compounds.

The maximum $5 \%$ weight loss temperature was observed for compound $\mathrm{LH} 1$ at $355^{\circ} \mathrm{C}$ and the minimum was observed for pure PLA at $350^{\circ} \mathrm{C}$. Similarly, the maximum $50 \%$ weight loss temperature was observed for compound $\mathrm{LH} 1$ at $386^{\circ} \mathrm{C}$ and minimum was observed for pure PLA at $381^{\circ} \mathrm{C}$. There was no significant difference in the temperature range over which these weight losses occurred. Since the TGA could only measure the weight loss in the form of the released volatile gases, we characterized the degradation leading to molecular weight reduction and oligomer formation by GPC (Section "Effects of additives on polymer degradation") and rheological analysis (Section "Effects of additives on melt viscosity").

\section{Effects of additives on melt viscosity}

The shear viscosities of pure PLA and its compounds at a set temperature of $200^{\circ} \mathrm{C}$ and a shear rate of $5 \mathrm{rad} / \mathrm{s}$ are summarized in Figure 4.23 . The viscosity of the polymer melt increased by $\sim 22 \%$ following the addition of alizarin, from $493 \mathrm{~Pa} \cdot \mathrm{s}$ (PLA) to $601 \mathrm{~Pa} \cdot \mathrm{s}$ (A1) and $595 \mathrm{~Pa} \cdot \mathrm{s}(\mathrm{A} 2)$. Higher melt viscosity tends to increase fiber diameters during melt electrospinning, but narrower fibers can still be achieved if the additives increase the electrical conductivity of the melt [19]. The addition of quercetin had a plasticizing effect on the PLA and reduced the melt viscosity by $\sim 37 \%$, from $493 \mathrm{~Pa} \cdot \mathrm{s}$ 
(PLA) to $308 \mathrm{~Pa} \cdot \mathrm{S}$ (Q1) and $312 \mathrm{~Pa} \cdot \mathrm{S}$ (Q2). However, the addition of hematoxylin achieved the most dramatic effect, reducing the melt viscosity by $\sim 91 \%$ at both concentrations, to $42 \mathrm{~Pa} \cdot \mathrm{s}$. The melting point of hematoxylin $\left(200^{\circ} \mathrm{C}\right)$ is much lower than that of the other dyes, so the low viscosity of the compounds containing hematoxylin may reflect the melting of the dye along with the polymer. The other possible hypothesis for reducing the viscosity of both hematoxylin and quercetin compounds is polymer degradation. The degradation hypothesis was addressed by GPC analysis (Section "Effects of additives on polymer degradation").

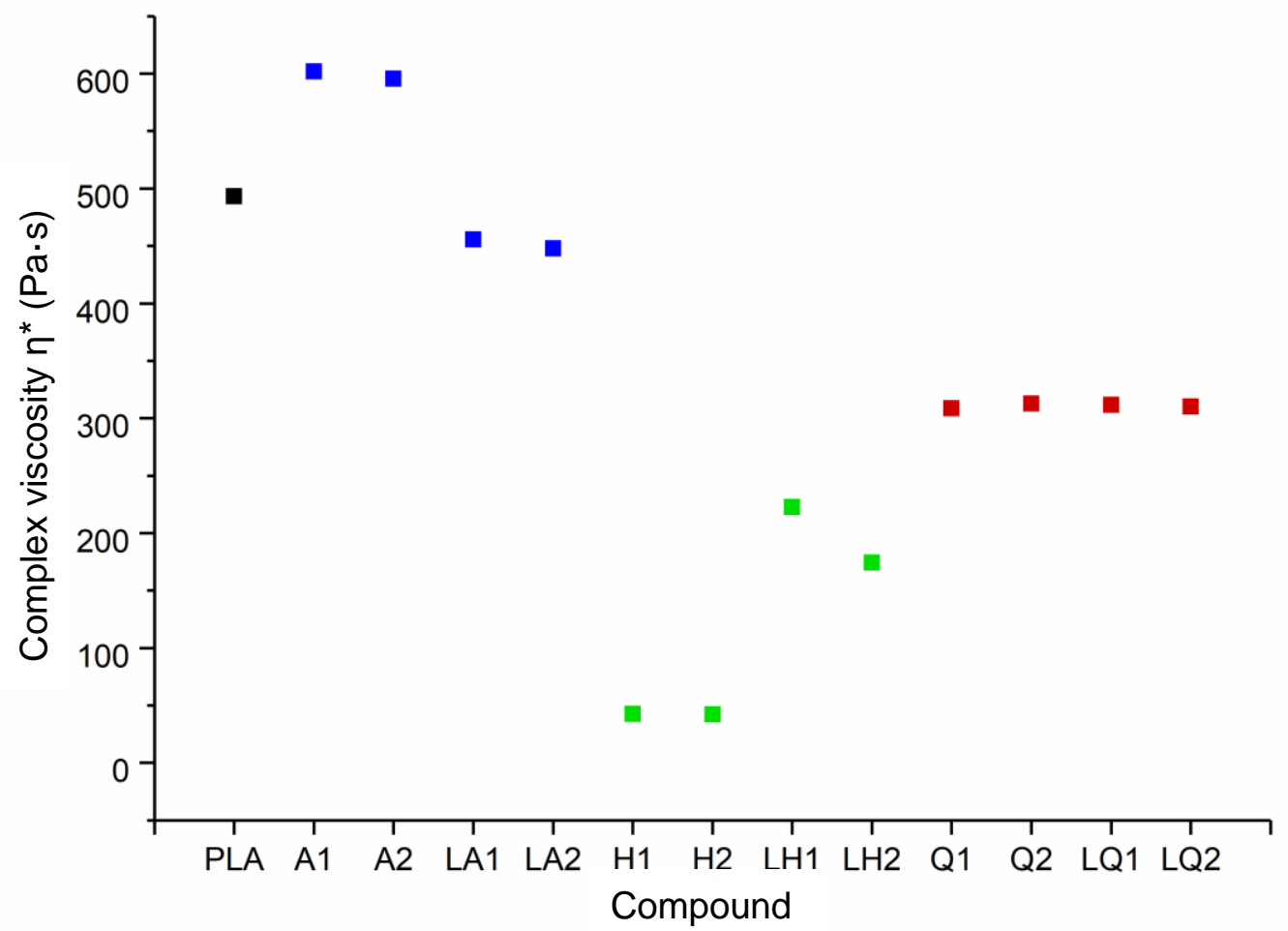

Fig. 4.23: Shear viscosity of pure PLA and the PLA/dye compounds, corresponding to a set temperature of $200^{\circ} \mathrm{C}$ and a shear rate of $5 \mathrm{rad} / \mathrm{s}$.

Overall, there was little difference in viscosity between compounds containing $1 \%$ or $2 \%(w / w)$ of a given dye. This was not the case for the liquid dyes, where the plasticizer showed a significant concentration-dependent effect. In the case of alizarin, the dye itself increased the melt viscosity whereas the plasticizer has the opposite effect, so the combination showed only a slight reduction in viscosity compared to pure PLA (7\% for LA1 and 9\% for LA2). In contrast, because hematoxylin and quercetin reduced the melt viscosity, the addition of plasticizer was expected to enhance this effect. Surprisingly, this was not the case - the viscosity of the liquid hematoxylin and quercetin compounds was significantly higher than the compounds prepared with pure dyes taking into account that also the dye concentration is significantly lower. For example, the viscosity of Q1 was $30 \%$ lower than PLA, and the viscosity of $\mathrm{H} 1$ was $81 \%$ lower than $\mathrm{LH} 1$. Normally the polar group of the plasticizer interacts with the polar group of the polymer, swelling the polymer chains and increasing the free volume. Such interactions would reduce 
intermolecular cohesion and increase polymer chain mobility, thus reducing the viscosity of the melt [46]. The unexpected behavior of the quercetin and hematoxylin compounds may reflect a stronger interaction between these dyes and the plasticizer compared to the interaction between the polymer and plasticizer. The interaction between dye and plasticizer molecules generates bulky particles that could hinder the motion of the polymer chains and ultimate increase the viscosity. Since the three dyes used are chemically different, they interact differently with the plasticizer.

\section{Effect of additives on polymer degradation}

GPC analysis of PLA and its compounds (Figure 4.24) revealed $M_{w}, M_{n}$ and PDI values of $184,000 \mathrm{~g} / \mathrm{mol}, 107,000 \mathrm{~g} / \mathrm{mol}$ and 1.72 , respectively, for pure PLA. There was no significant change from these values in the alizarin and liquid alizarin compounds. This confirms that the increase in $\mathrm{X}_{\mathrm{c}}$ observed for the alizarin and liquid alizarin compounds is a result of the nucleating effect of alizarin. In the quercetin and liquid quercetin compounds, there was a slight reduction in $\mathrm{M}_{\mathrm{w}}$ and $\mathrm{M}_{\mathrm{n}}$. A more apparent change was observed in compound $Q 2$, where the $M_{w}$ and $M_{n}$ values were $15.12 \%$ and $14.01 \%$ lower, respectively, compared to pure PLA. The PDI increased for both LQ1 and LQ2, and the highest PDI was observed for LQ2. However, since the change in molecular weight is not as drastic as for the hematoxylin compounds, the viscosity of both quercetin and liquid quercetin compounds was observed to be similar. Hence, in this case, the increase in crystallinity of LQ1 and LQ2 can be attributed to the fact that a lower quantity of quercetin was present compared to Q1 and Q2. In the hematoxylin compounds, the changes were more significant. For example, the $\mathrm{M}_{\mathrm{w}}$ and $\mathrm{M}_{\mathrm{n}}$ of compound $\mathrm{H} 2$ decreased by $40.71 \%$ and $40.74 \%$, respectively, compared to pure PLA. As the percentage losses in $M_{w}$ and $M_{n}$ were the same, their PDI ratio remained unaffected. The GPC measurements confirmed that the degradation of compounds containing hematoxylin led to the observed reduction in viscosity and increase in $\mathrm{X}_{\mathrm{c}}$ (Section "Effects of additives on melt viscosity"). However, in case of the liquid hematoxylin compounds, the $M_{w}$ and $M_{n}$ are higher compared to the $\mathrm{M}_{\mathrm{w}}$ and $\mathrm{M}_{\mathrm{n}}$ of the hematoxylin compounds. The hematoxylin content of $\mathrm{LH} 1$ and $\mathrm{LH} 2$ compounds is only $25 \%(\mathrm{w} / \mathrm{w})$ compared to that of $\mathrm{H} 1$ and $\mathrm{H} 2$ compounds. This could have led to lesser degradation. Furthermore, the interaction between the dye and the plasticizer might have led to bulky particles hindering the flow of polymer chains. This combined effect could have led to higher viscosity reported for these compounds in the previous section. 


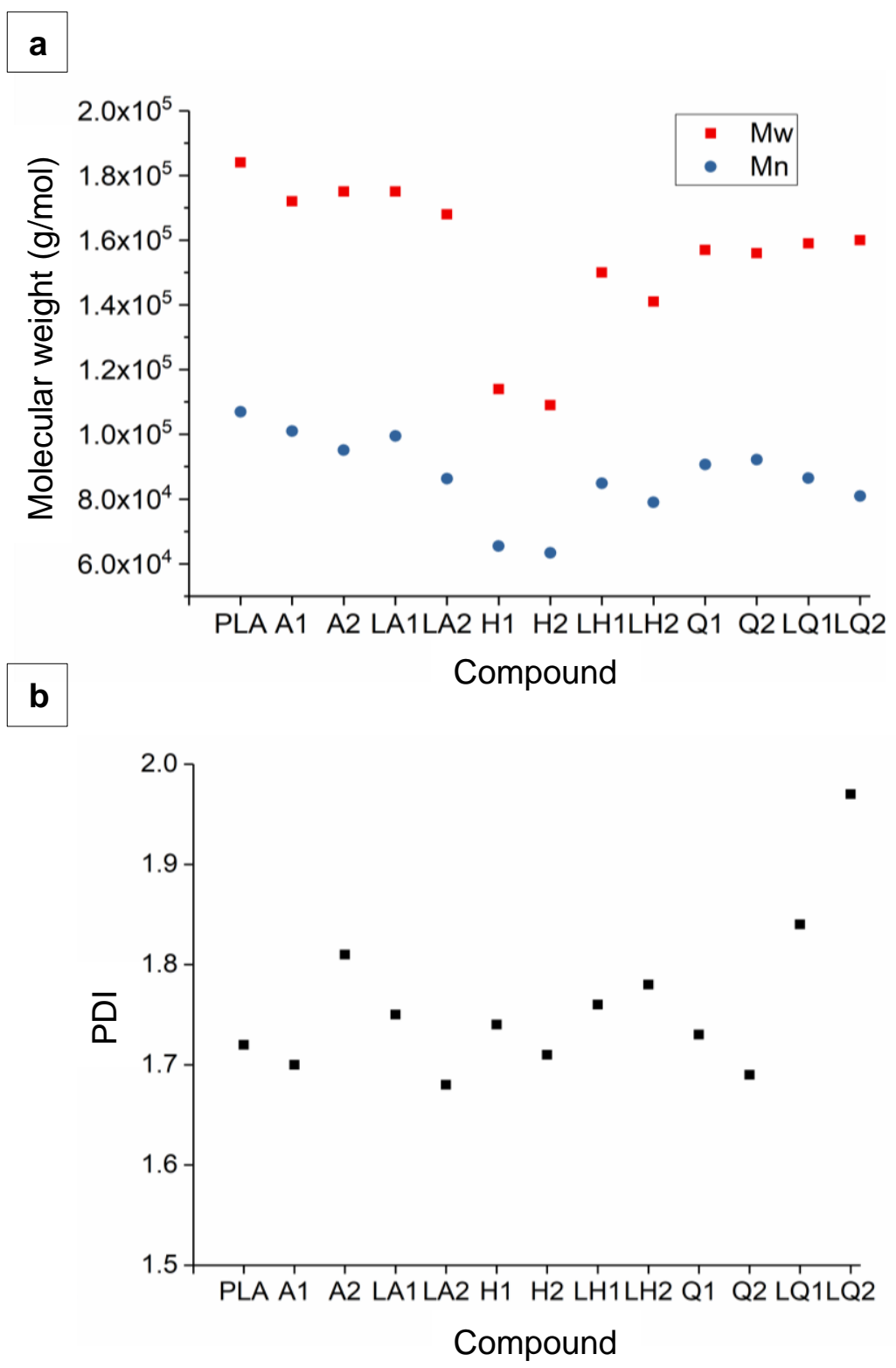

Fig. 4.24: Molecular weight (a) and PDI (b) of PLA and the PLA/dye compounds.

GPC analysis was also carried out on the melt-electrospun fibers, but no significant reduction of the molecular weight could be detected due the short dwell time of the polymer melt in the syringe of less than less than thirty seconds (Appendix: Fig. A5).

\section{Effect of additives on melt conductivity}

The electrical resistance of pure PLA its compounds was measured at a set temperature of $325^{\circ} \mathrm{C}$ (Figure 4.25). The higher temperature compared to the spinning process was chosen because more heat energy is lost over the larger surface of the beaker and thus more energy must be supplied to achieve the same melting conditions. However, it should be taken into account that the dyes could melt at the selected temperature and this would most likely influence the electrical conductivity behavior. Electrical conductivity requires freely movable charge carriers, so the electrical resistance of pure PLA (5.0 G $\Omega$ ) 
decreased by a factor of five in the presence of any of the additives. In the compounds containing dyes but no plasticizer, the electrical resistance was also inversely related to the dye concentration. Because alizarin increased the viscosity of the melt, the higher conductivity is likely to favor the melt-electrospinning process and reduce the fiber diameter. Furthermore, electrical resistance in the liquid alizarin compounds was lower than in compounds containing alizarin but no plasticizer, indicating that the plasticizer also contributes to the higher conductivity, as reported in earlier studies [47, 48]. We observed similar behavior in the liquid hematoxylin and liquid quercetin compounds, although the overall effect of quercetin on electrical conductivity was weakest. The synergetic effects of viscosity and conductivity on fiber diameter are described in more detail in Section "Fiber diameters achieved using different PLA compounds".

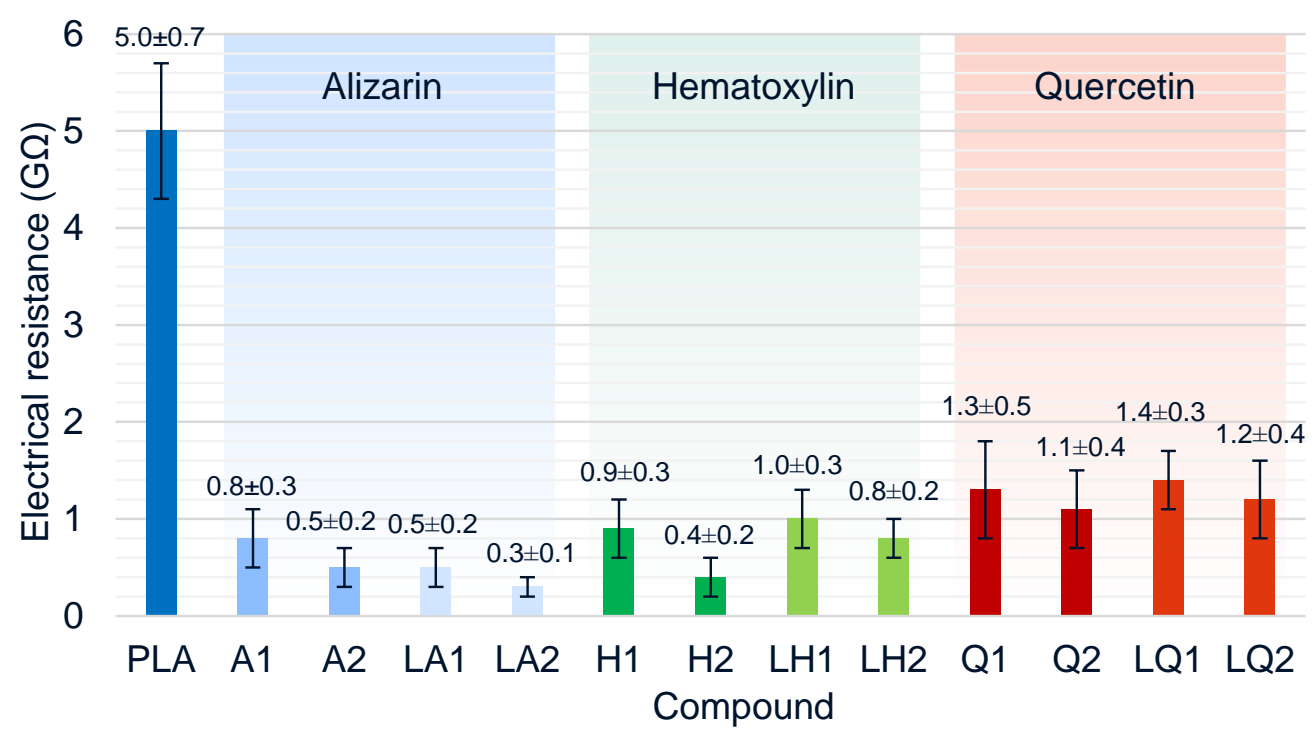

Fig. 4.25: Electrical resistances of pure PLA and PLA/dye compounds.

\section{Physical properties of PLA fibers under different processing conditions}

The physical properties of the melt-spun PFs and DFs were compared to the meltelectrospun PLA fibers by DSC (Table 4.8) and the corresponding thermograms are presented in Figure 4.26.

Tab. 4.8: Properties of PLA fibers, comparing partially orientated and drawn filaments with melt-electrospun fibers.

\begin{tabular}{|l|l|l|l|l|}
\hline Material & $\mathbf{T}_{\mathbf{g}}\left({ }^{\circ} \mathbf{C}\right)$ & $\mathbf{T}_{\mathbf{c c}}\left({ }^{\circ} \mathbf{C}\right)$ & $\mathbf{T}_{\mathbf{m}}\left({ }^{\circ} \mathbf{C}\right)$ & $\mathbf{X}_{\mathbf{c}}(\%)$ \\
\hline Partially-oriented PLA filament & 62.90 & 95.70 & 174.10 & 25.26 \\
\hline Drawn PLA filament & - & - & 175.70 & 58.83 \\
\hline Melt-electrospun PLA fiber & 62.10 & 108.90 & 174.30 & 8.87 \\
\hline
\end{tabular}




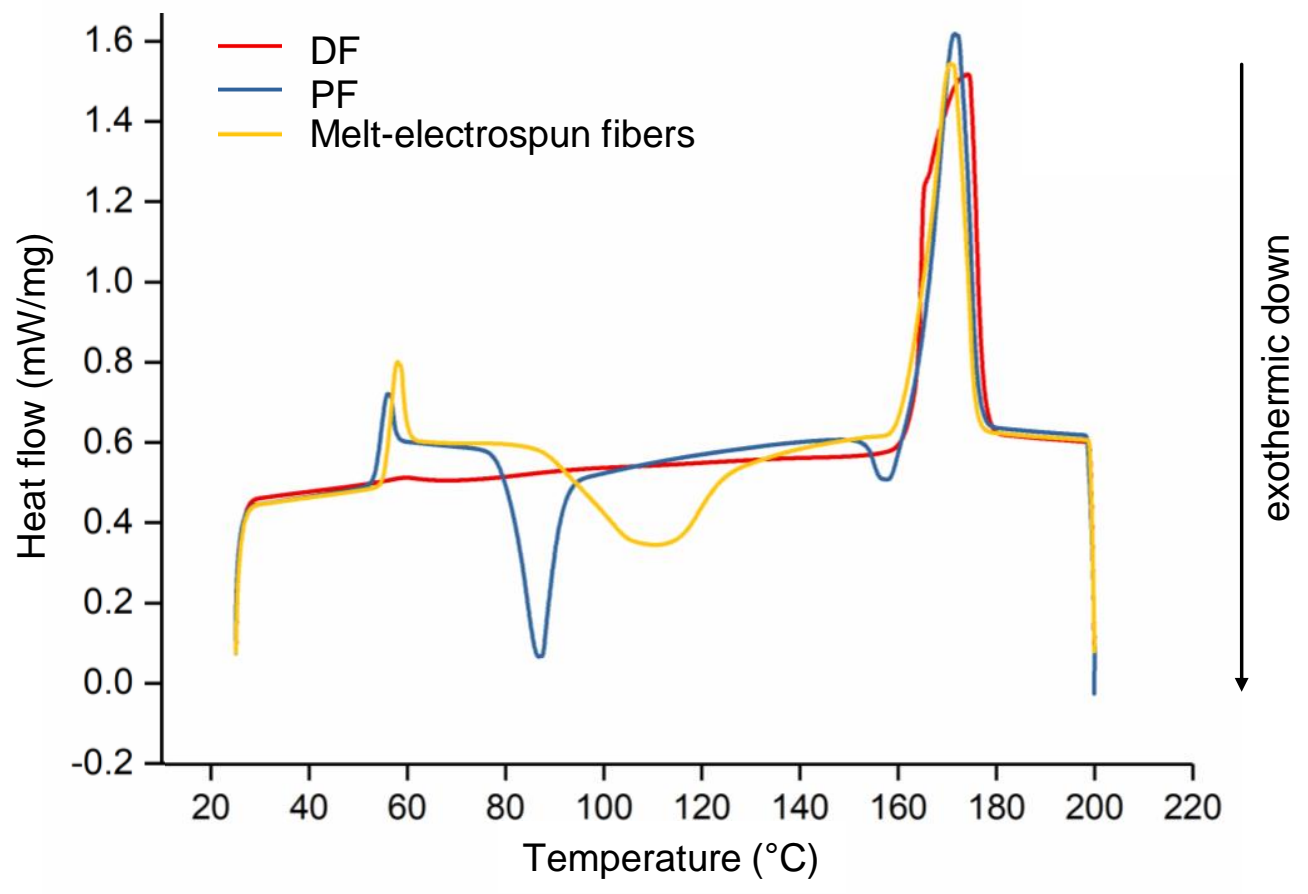

Fig. 4.26: DSC thermograms of partially-orientated filaments, drawn filaments and melt-electrospun PLA fibers.

The $T_{g}, T_{m}$ and $T_{c c}$ values were similar for the PFs and melt-electrospun fibers, but we were unable to determine $T_{g}$ or $T_{c c}$ values after fiber drawing. Furthermore, the $X_{c}$ of the PFs and melt-electrospun fibers were very low (17.93\% and $8.72 \%$, respectively), whereas the $X_{c}$ after fiber drawing was $58.83 \%$. The absence of a glass transition in DSC thermograms often occurs when the crystalline fraction is more abundant than the amorphous fraction, and the $T_{c c}$ value is absent because the DF is fully drawn, in agreement with previous studies [49]. The melt-electrospun fibers are therefore more similar to a PF than a DF.

POM analysis of the melt-electrospun fibers, PFs and DFs revealed the extent of crystallinity compared to pure PLA fibers produced under different processing conditions (Figure 4.27). Optically anisotropic materials such as crystalline materials give rise to birefringence due to the difference in their axis length [50]. Although individual crystals could not be observed by POM, very little to no birefringence was detected in the meltelectrospun fibers and PFs, whereas birefringence was observed in the DFs because the degree of crystallinity was increased by drawing. The same technique was previously used to show that the degree of crystallinity in PA66 tensile bars was dependent on the mold temperature [51]. Our combined DSC and POM data therefore indicate that the PLA fibers produced by melt electrospinning are similar to PFs produced by melt spinning, which have a much lower $T_{c c}$ value than DFs. 

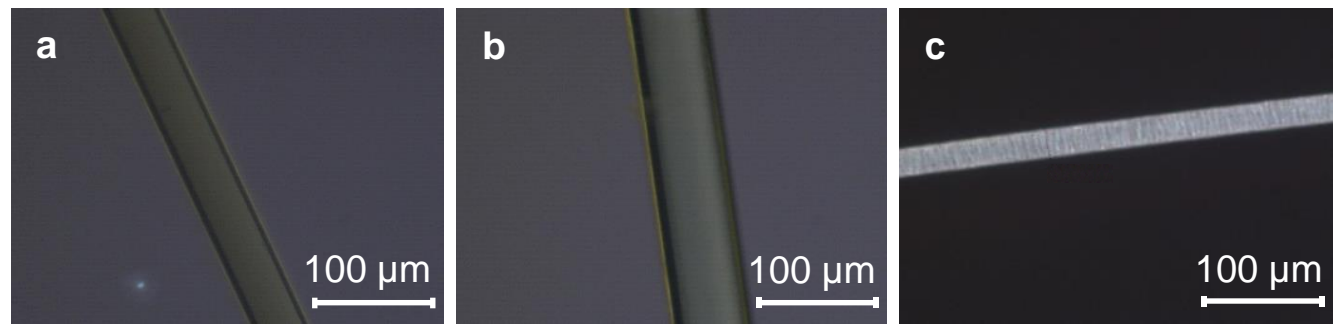

Fig. 4.27: POM images. (a) Melt-electrospun PLA fiber. (b) Partially-oriented PLA filament. (c) Drawn PLA filament.

\section{Fiber diameters achieved using different PLA compounds}

The processability of the compounds and the influence of additives on the fiber diameter were investigated by producing fibers using a single-fiber melt-electrospinning device. For pure PLA, the formation of a Taylor cone followed by typical fiber deposition was observed at a set temperature of $275^{\circ} \mathrm{C}$. Fiber formation was possible with all compounds at this temperature and the corresponding fiber diameters could therefore be determined under the same conditions.

The average fiber diameter for pure PLA was $70.6 \mu \mathrm{m}$ (Figure 4.28). All compounds produced thinner fibers, indicating that all the additives affected viscosity and/or conductivity in a beneficial manner.

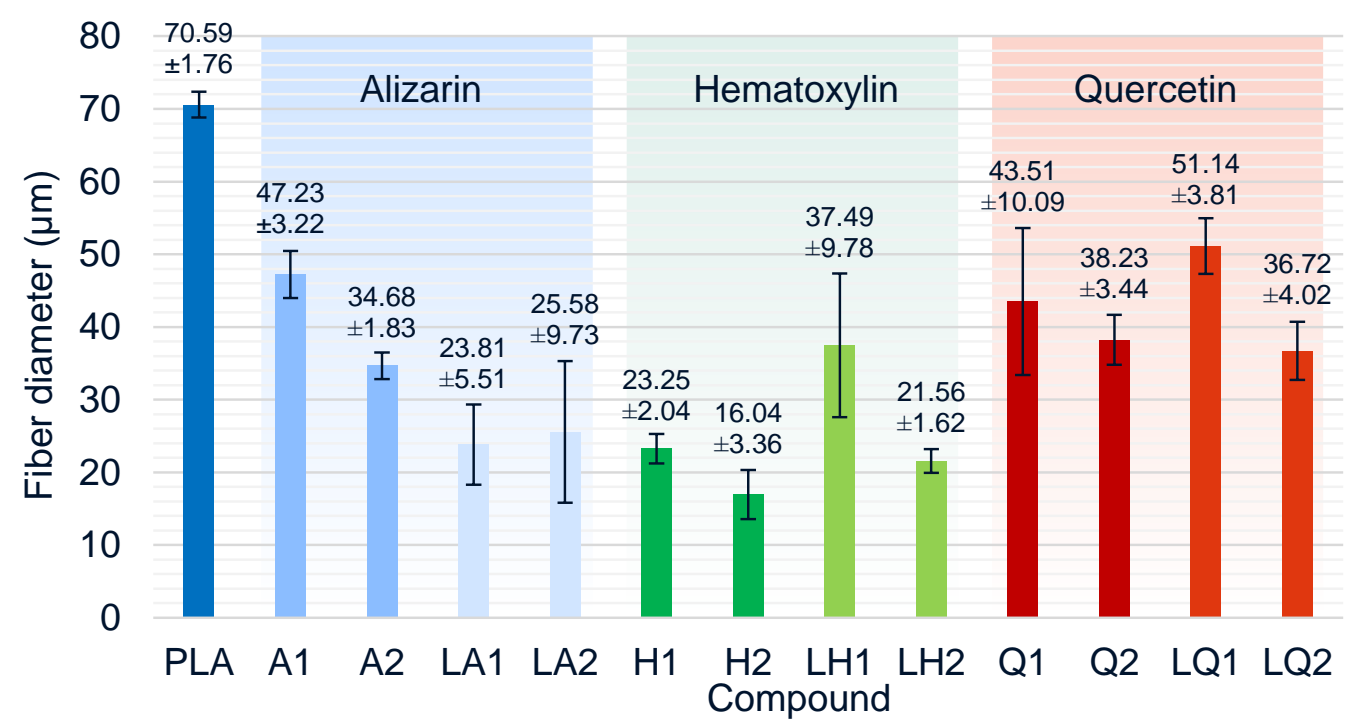

Fig. 4.28: Fiber diameters and standard deviations of PLA and the PLA/dye compounds produced by melt electrospinning at $275^{\circ} \mathrm{C}$ using a singlenozzle laboratory device.

Despite the increase in viscosity caused by the addition of alizarin, the fiber diameters of compounds $A 1$ and $A 2$ were reduced by $33 \%$ and $50 \%$ respectively, compared to pure PLA. The increase in electrical conductivity conferred by alizarin therefore compensated for the increase in viscosity and the influence of conductivity was dominant, especially at higher alizarin concentrations. The presence of plasticizer in addition to alizarin reduced 
the viscosity of the melt, and the smallest fiber diameter of all alizarin compounds was therefore achieved by LA1 $(23.8 \mu \mathrm{m}, 63 \%$ narrower than pure PLA). There was no significant difference between the fiber diameters of LA1 and LA2.

The addition of hematoxylin led to a significant reduction in viscosity, and the degradation of the polymer (and thus a reduction in $\mathrm{M}_{\mathrm{w}}$ ) was detected by GPC. As expected, this resulted in the most profound reduction in fiber diameter among all compounds. The finest fibers ( $16.04 \mu \mathrm{m}, 77 \%$ narrower than pure PLA) were achieved for compound $\mathrm{H} 2$. The increase in fiber diameter in the presence of the plasticizer matched the unexpected increase in viscosity of liquid hematoxylin compared to hematoxylin compounds without a plasticizer, and the fall in electrical conductivity due to the overall lower concentration of hematoxylin when the plasticizer was present.

Finally, the effect of quercetin on fiber diameter was similar to that of alizarin when each dye was presented in the absence of plasticizer. Interestingly, the dyes had opposite effects in the presence of plasticizer, with the liquid alizarin compounds LA1 and LA2 reducing the fiber diameter further than compounds $A 1$ and $A 2$, but the liquid quercetin compounds LQ1 and LQ2 producing fibers that were similar in diameter or thicker than those based on compounds Q1 and Q2. As discussed above, this mirrors the opposing effects on viscosity: the plasticizer reduced the viscosity of compounds containing alizarin but increased the viscosity of those containing quercetin. These data also suggest that quercetin has a less significant effect on conductivity than alizarin.

We plotted the relationship between the electrical resistance, melt viscosity and average fiber diameter using Minitab 19 analysis software. Figure 4.29 presents surface plots of fiber diameters in relation to the electrical resistance and viscosity of the alizarin, hematoxylin and quercetin compounds. It has to be considered that the set temperature of the viscosity and resistance measurement deviates from the set temperature of the spinning process, as explained in Section "Characterization of compounds", so that only a trend can be described. As the temperature increases, the viscosity decreases as well as the resistance [34], so that a further reduction of the fiber diameters is to be expected, The fibers prepared from all three compounds became finer with decreasing melt viscosity and electrical resistance. For the alizarin compounds, the increasing conductivity was the decisive factor controlling fiber diameter because there was little variation in viscosity. The minimum fiber diameter was always achieved using compounds with the lowest viscosity and the lowest electrical resistance. 
a

Fiber diameter $(\mu \mathrm{m})$

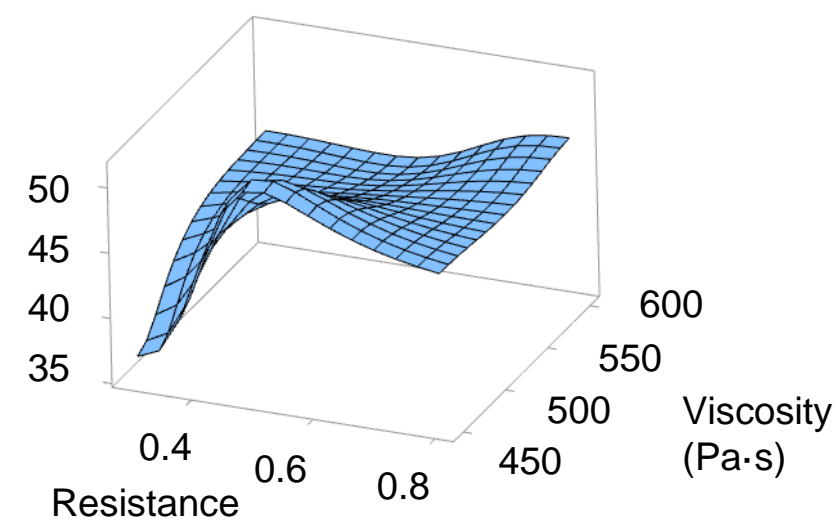

(G $\Omega$ )

b

Fiber diameter ( $\mu \mathrm{m})$

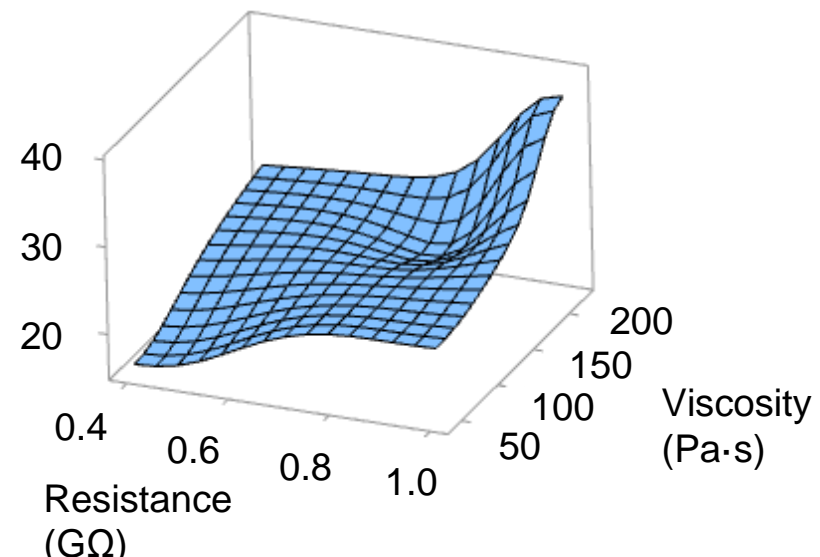

C

Fiber diameter $(\mu \mathrm{m})$

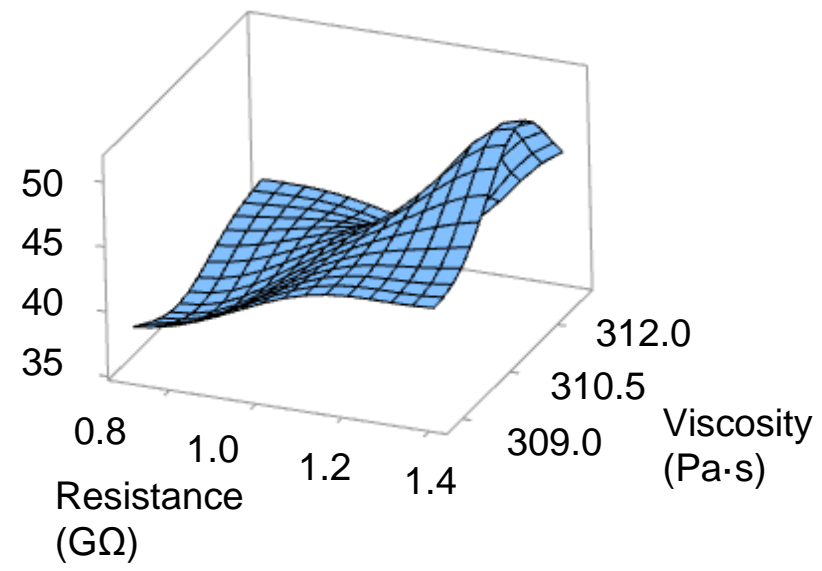

Fig. 4.29: Surface plots of fiber diameters in relation to the electrical resistance and viscosity of the melt. (a) PLA/alizarin compounds. (b) PLA/hematoxylin compounds. (c) PLA/quercetin compounds.

The fiber diameters we have achieved are still in the micrometer range, like the majority of fiber diameters previously reported in literature. However, it should be stated that we have not made any device-specific modifications, such as the integration of an accelerated airflow or a heating system as it is already used for other meltelectrospinning systems [27]. Furthermore, the fiber diameter is strongly influenced by the flow rate of the polymer [1], so that the use of a nozzle with a smaller orifice can significantly reduce the fiber diameter in future attempts. 


\section{Conclusion and further perspectives}

We have successfully tested the biobased dyes alizarin, hematoxylin and quercetin as conductive additives in the melt-electrospinning process, and have produced fibers in the micrometer range. All dyes and dye/plasticizer combinations contributed to the desirable reduction of fiber diameter compared to pure melt-electrospun PLA fibers, which will facilitate the development of an economical and environmentally friendly process for the production of microfibers and nanofibers that could ultimately replace solution electrospinning. The formation of a Taylor cone followed by continuous fiber deposition was observed for all dyes and dye/plasticizer combinations. The finest fibers (16.04 $\mu \mathrm{m}$ in diameter) were produced by adding $2 \%(\mathrm{w} / \mathrm{w})$ hematoxylin, reducing the average fiber diameter by $77 \%$ compared to pure PLA. However, hematoxylin induced polymer degradation at a spinning temperature of $275^{\circ} \mathrm{C}$, which reduces the $\mathrm{M}_{w}$ and therefore favors the production of finer fibers. In future experiments, the process temperature should be lowered when using hematoxylin to prevent degradation. The addition of alizarin produced finer fibers than pure PLA despite the increase in melt viscosity, indicating that alizarin has a profound effect on the electrical conductivity of the melt. A combination of alizarin (to increase conductivity) and a plasticizer (to reduce viscosity) reduced the fiber diameter to $23.8 \mu \mathrm{m}$, which is $63 \%$ narrower than the pure PLA fibers. The addition of quercetin reduced the melt viscosity but had a limited effect on electrical conductivity compared to alizarin, and the finest fibers containing this additive (achieved by adding $2 \%(\mathrm{w} / \mathrm{w}$ ) liquid quercetin) were $36.72 \mu \mathrm{m}$ in diameter. The analysis of fibers produced by melt spinning, melt spinning with post-drawing, and melt electrospinning revealed that the melt-electrospun fibers had a similar degree of crystallinity to PFs and are not comparable to drawn filaments.

\section{Acknowledgements}

The authors acknowledge support from the Microscopy Department of the Institute for Textile Technology, RWTH Aachen University, Aachen, Germany. We would also like to thank our laboratory technician Henri Becker for the maintenance and modification of our equipment, and our student Konrad Beukenberg for supporting the experimental trial preparations. 


\section{References}

[1] Brown T, Dalton P, Hutmacher DW. Melt electrospinning today: an opportune time for an emerging polymer process. Elsevier. 2016; 56.

[2] Huang Z-M, Zhang YZ, Kotaki M, Ramakrishna S. A review on polymer nanofibers by electrospinning and their applications in nanocomposites. Composites Science and Technology. 2003; 63(15):2223-53.

[3] Greiner A, Wendorff JH. Electrospinning: A Fascinating Method for the Preparation of Ultrathin Fibers. Angewandte Chemie International Edition. 2007; 46(30):5670703.

[4] Ma B, Xie J, Jiang J. Rational design of nanofiber scaffolds for orthopedic tissue repair and regeneration. Nanomedicine (Lond). 2013; 8.

[5] Shin S-H, Purevdorj O, Castano O. A short review: recent advances in electrospinning for bone tissue regeneration. J Tissue Eng. 2012; 3.

[6] McClellan P, Landis WJ. Recent applications of coaxial and emulsion electrospinning methods in the field of tissue engineering. Biores Open Access. 2016; 5.

[7] Khajavi R, Abbasipour M, Bahador A. Electrospun biodegradable nanofibers scaffolds for bone tissue engineering. J Appl Polym Sci. 2016; 133.

[8] Mani MP, Jaganathan SK, Ismail AF. Appraisal of electrospun textile scaffold comprising polyurethane decorated with ginger nanofibers for wound healing applications. Journal of Industrial Textiles. 2019; 49(5):648-62.

[9] Sundarrajan S, Tan KL, Lim SH. Electrospun nanofibers for air filtration applications. Procedia Eng. 2014; 75.

[10] Scholten E, Bromberg L, Rutledge GC. Electrospun polyurethane fibers for absorption of volatile organic compounds from air. ACS Appl Mater Interfaces. $2011 ; 3$.

[11] Wang J, Li Y, Sun X. Challenges and opportunities of nanostructured materials for aprotic rechargeable lithium-air batteries. Nano Energy. 2013; 2.

[12] Wang $\mathrm{X}$, Drew C, Lee S-H. Electrospun Nanofibrous membranes for highly sensitive optical sensors. Nano Lett. 2002; 2.

[13] Nan N, He J, You X, Sun X, Zhou Y, Qi K, et al. A Stretchable, Highly Sensitive, and Multimodal Mechanical Fabric Sensor Based on Electrospun Conductive Nanofiber Yarn for Wearable Electronics. Advanced Materials Technologies. 2019; 4(3):1800338.

[14] Bhat GS. Advances in polymeric nanofiber manufacturing technologies. J Nanomater Mol Nanotechnol. 2016; 5. 
[15] Bhardwaj N, Kundu SC. Electrospinning: a fascinating fiber fabrication technique. Biotechnol Adv. 2010; 28.

[16] Ogata N, Lu G, Iwata T, Yamaguchi S, Nakane K, Ogihara T. Effects of ethylene content of poly(ethylene-co-vinyl alcohol) on diameter of fibers produced by meltelectrospinning. Journal of Applied Polymer Science. 2007; 104(2):1368-75.

[17] Ogata N, Yamaguchi S, Shimada N, Lu G, Iwata T, Nakane K, et al. Poly(lactide) nanofibers produced by a melt-electrospinning system with a laser melting device. Journal of Applied Polymer Science. 2007; 104(3):1640-5.

[18] Zhou H, Green TB, Joo YL. The thermal effects on electrospinning of polylactic acid melts. Polymer (Guildf). 2006; 47.

[19] Nayak R, Kyratzis IL, Truong YB, Padhye R, Arnold L. Melt-electrospinning of polypropylene with conductive additives. Journal of Materials Science. 2012; 47(17):6387-96.

[20] Cadar O, Paul M, Roman C, Miclean M, Majdik C. Biodegradation behaviour of poly(lactic acid) and (lactic acid-ethylene glycol-malonic or succinic acid) copolymers under controlled composting conditions in a laboratory test system. Polymer Degradation and Stability. 2012; 97(3):354-7.

[21] Qin Y, Cheng L, Zhang Y, Chen X, Wang X, He X, et al. Efficient preparation of poly(lactic acid) nanofibers by melt differential electrospinning with addition of acetyl tributyl citrate. Journal of Applied Polymer Science. 2018; 135(31):46554.

[22] Hacker C, Fourne R, Rübsam $U$, et al. Challenges of the meltelectrospinning process: an economical and technical window of opportunity [Herausforderungen des Schmelzelektrospinns: wirtschaftliche und technische Potentiale und Möglichkeiten]. Austria: Österreichisches Chemiefaser-Institut, 2013.

[23] Nayak R. Polypropylene nanofibers: melt electrospinning versus Meltblowing. Engineering Material: Springer International Publishing; 2017.

[24] Nayak R. Effect of viscosity and electrical conductivity on the morphology and fiber diameter in melt electrospinning of polypropylene. Textile Research Journal. 2013; 83(6):606.

[25] Maqsood M, Langensiepen F, Seide G. Investigation of melt spinnability of plasticized polylactic acid biocomposites-containing intumescent flame retardant. Journal of Thermal Analysis and Calorimetry. 2020; 139(1):305-18.

[26] Yoon YI, Park KE, Lee SJ, Park WH. Fabrication of Microfibrous and Nano/Microfibrous Scaffolds: Melt and Hybrid Electrospinning and Surface Modification of Poly(L-lactic acid) with Plasticizer. BioMed Research International. 2013; 10.

[27] Zhmayev E, Cho D, Joo YL. Nanofibers from gas-assisted polymer melt electrospinning. Polymer. 2010; 51(18):4140-4. 
[28] Liu Y, Zhao F, Zhang C, Zhang J, Yang W. Solvent-free preparation of poly(lactic acid) fibers by melt electrospinning using an umbrella-like spray head and alleviation of the problematic thermal degradation. J Serb Chem Soc. 2012; 77.

[29] Carroll CP, Zhmayev E, Kalra V, Joo YL. Nanofibers from electrically driven viscoelastic jets: modeling and experiments. Korea-Australia Rheology Journal. 2008; 20(3):153-64.

[30] Chrzanowska O, Struszczyk M, Krucińska I. Small Diameter Tubular Structure Design Using Solvent-Free Textile Techniques. Journal of Applied Polymer Science. 2014; 131.

[31] Mazalevska O, Struszczyk M, Chrzanowski M, Krucińska I. Application of Electrospinning for Vascular Prothesis Design-Preliminary Results. Fibres and Textiles in Eastern Europe. 2011; 19:46-52.

[32] Mazalevska O, Struszczyk MH, Krucinska I. Design of vascular prostheses by melt electrospinning -structural characterizations. Journal of Applied Polymer Science. 2013; 129(2):779-92.

[33] Zhmayev E, Zhou H, Joo YL. Modeling of non-isothermal polymer jets in melt electrospinning. Journal of Non-Newtonian Fluid Mechanics. 2008; 153(2):95-108.

[34] Koenig K, Daenicke J, Langensiepen F, Seide G, Schubert DW. From lab to pilot scale: melt electrospun nanofibers of polypropylene with conductive additives. J Nanomater Mol Nanotechnol. 2019; 8(2).

[35] Alves de Lima RO, Bazo AP, Salvadori DMF, Rech CM, de Palma Oliveira D, de Aragão Umbuzeiro G. Mutagenic and carcinogenic potential of a textile azo dye processing plant effluent that impacts a drinking water source. Mutation Research/Genetic Toxicology and Environmental Mutagenesis. 2007; 626(1):5360.

[36] Buccella M. Color masterbatches for polyamide 6 fibers. Optimization of compounding and spinning processes. Physical-chemical characterization of industrial products: University of Trento; 2014.

[37] Sun C, Li Y, Qi D, Li H, Song P. Optical and electrical properties of purpurin and alizarin complexone as sensitizers for dye-sensitized solar cells. Journal of Materials Science: Materials in Electronics. 2016; 27(8):8027-39.

[38] Gsänger M, Bialas D, Huang L, Stolte M, Würthner F. Organic Semiconductors based on Dyes and Color Pigments. Advanced Materials. 2016; 28(19):3615-45.

[39] Jia S, Yu D, Zhu Y, Wang Z, Chen L, Fu L. Morphology, Crystallization and Thermal Behaviors of PLA-Based Composites: Wonderful Effects of Hybrid GO/PEG via Dynamic Impregnating. Polymers. 2017; 9(10):528. 
[40] Cole D. Bringing Distillation \& Solvent Recovery In House: FlexoGlobal; 2019 https://www.flexoglobal.com/blog-articles/2019/daetwyler-01-bringing-distillationand-solvent-recovery-in-house.html (accessed 21 December 2019).

[41] Maqsood M, Seide G. Novel Bicomponent Functional Fibers with Sheath/Core Configuration Containing Intumescent Flame-Retardants for Textile Applications. Materials. 2019; 12(19):3095.

[42] Lascano D, Quiles-Carrillo L, Balart R, Boronat T, Montanes N. Toughened Poly (Lactic Acid)_PLA Formulations by Binary Blends with Poly(Butylene Succinateco-Adipate)_PBSA and Their Shape Memory Behaviour. Materials. 2019; 12(4):622.

[43] Broda J. Nucleating activity of the quinacridone and phthalocyanine pigments in polypropylene crystallization. Journal of Applied Polymer Science. 2003; 90(14):3957-64.

[44] Mubarak Y, Martin PJ, Harkin-Jones E. Effect of nucleating agents and pigments on crystallisation, morphology, and mechanical properties of polypropylene. Plastics, Rubber and Composites. 2000; 29(7):307-15.

[45] Sukata K, Takeuchi H, Shimada M, Agari Y. Influence of the nigrosine dye on the thermal behavior of polyamide 66. Journal of Applied Polymer Science. 2006; 101(5):3270-4.

[46] Marcilla A, BeltrÁN M. 5 - MECHANISMS OF PLASTICIZERS ACTION. In: Wypych G, editor. Handbook of Plasticizers (Second Edition). Boston: William Andrew Publishing; 2012. p. 119-33.

[47] Yang XQ, Lee HS, Hanson L, McBreen J, Okamoto Y. Development of a new plasticizer for poly(ethylene oxide)-based polymer electrolyte and the investigation of their ion-pair dissociation effect. Journal of Power Sources. 1995;54(2):198-204.

[48] Reddy T, Achari V, Sharma A, Rao V. Effect of plasticizer on electrical conductivity and cell parameters of (PVC+KBrO3) polymer electrolyte system. Ionics. 2007; 13:55-9.

[49] Walker J, Melaj M, Giménez R, Pérez E, Bernal C. Solid-State Drawing of Commercial Poly(Lactic Acid) (PLA) Based Filaments. Frontiers in Materials. 2019; 6(280).

[50] Bergström J. 2 - Experimental Characterization Techniques. In: Bergström J, editor. Mechanics of Solid Polymers: William Andrew Publishing; 2015. p. 19-114.

[51] Drummer D, Seefried A, Meister S. Characterization of Material Stiffness on Injection Moulded Microspecimens Using Different Test Methods. Advances in Materials Science and Engineering. 2014. 


\section{Chapter 5}

\section{Integration of a climate system}

Pilot-scale production of polylactic acid nanofibers by melt electrospinning

K. Koenig, F. Langensiepen, G. Seide e-Polymers. 2020; 20: 233-241.

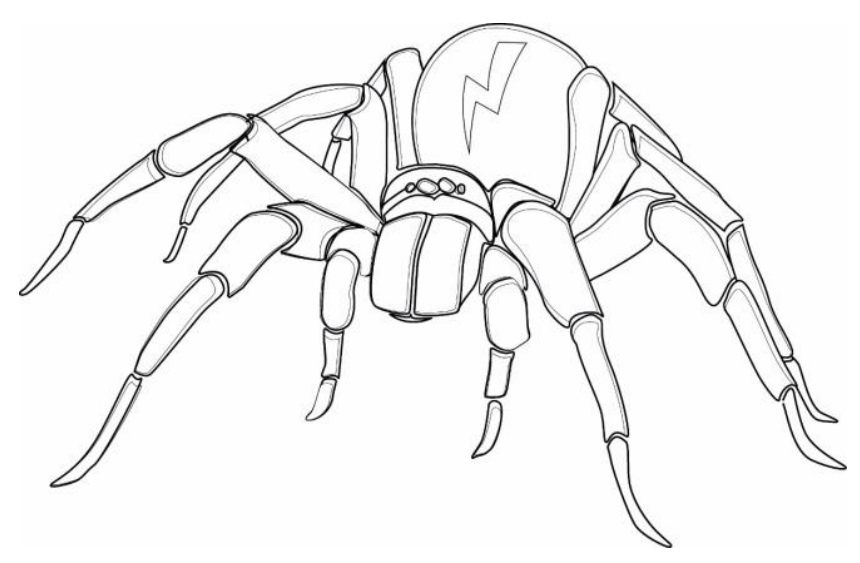




\title{
Pilot-scale production of polylactic acid nanofibers by melt electrospinning
}

\begin{abstract}
Melt electrospinning has been used to manufacture fibers with diameters in the low micrometer range but the production of sub-micrometer fibers has proven more challenging. In Chapter 5, we investigated the feasibility of fabricating polylactic acid nanofibers using polymer grades with increasing melt flow rates $(15-85 \mathrm{~g} / 10 \mathrm{~min}$ at $210^{\circ} \mathrm{C}$ ) by melt electrospinning with a 600-nozzle pilot-scale device featuring an integrated climate control system realized as a glass chamber around the spinneret. Previous experiments using this device without appropriate climate control produced fibers exceeding $1 \mu \mathrm{m}$ in diameter because the drawing of fibers was inhibited by the rapid cooling of the polymer melt. The integrated glass chamber created a temperature gradient exceeding the glass transition temperature of the polymer, which enhanced the drawing of fibers below the spinneret. An average fiber diameter of $810 \mathrm{~nm}$ was achieved using PLA Ingeo Biopolymer 6252, and the finest individual fiber (420 nm in diameter) was produced at a spin pump speed of $5 \mathrm{rpm}$ and a spinneret set temperature of $230^{\circ} \mathrm{C}$. We have therefore demonstrated the innovative performance of our pilot-scale meltelectrospinning device, which bridges the gap between laboratory-scale and pilot-scale manufacturing and achieves fiber diameters comparable to those produced by conventional solution electrospinning.
\end{abstract}

\section{Introduction}

Electrospinning is the most common method for the production of polymer fibers with diameters in the low micrometer to nanometer range $[1,2]$. There are two main variants of the method [1, 3]. In solution electrospinning, the polymer is dissolved in an organic solvent that evaporates as the fiber is spun. In melt electrospinning, the polymer is heated to above its melting point and solidifies as the fiber is spun. In both cases, a strong electric field is applied during spinning to stretch the polymer, typically resulting in fibers that are several hundred nanometers in diameter after drawing [4]. Solution electrospinning is the simplest of the two processes because it does not require high temperatures or coagulation chemistry, but a solvent recovery step must be included and there is a risk that toxic solvents may be carried over into the final product $[3,5]$. There are no such risks in melt electrospinning, and the entire process is therefore more environmentally sustainable [6]. However, due to the high viscosity and low electrical conductivity of the polymer melts, the resulting fibers have a slightly larger average diameter than those produced by solution electrospinning $[3,7]$. 
Industry currently favors solution electrospinning not only because it produces finer fibers but also because the method is more scalable than melt electrospinning, and fiber manufacturing processes are therefore more economical despite the need for solvent recovery $[3,6,8,9]$. To address the limited scalability of melt electrospinning, recent innovations such as multiple-needle and needleless device configurations have demonstrated a roadmap to overcome low flow rates (typically in the $\mu \mathrm{g} / \mathrm{h}$ range) and thus increase productivity [3,10]. Prototypes with umbellate nozzles containing 60 spinnerets can achieve maximum product deposition rates of $\sim 36 \mathrm{~g} / \mathrm{h}[11,12]$. A polymer melt differential electrospinning system with a linear slot spinneret achieved an output of $75.6 \mathrm{~g} / \mathrm{h}$, which was improved further by increasing the slot width [8]. Until recently, the largest multi-needle configuration was a device with 64 nozzles [13, 14], but in our previous report we described a prototype with 600 nozzles that provides the basis for pilot-scale melt electrospinning [15].

Although our prototype addresses the limited scalability of melt electrospinning, the resulting fibers are still coarser than those produced by solution electrospinning. Using a commercial spinning-grade polylactic acid (PLA) resin (Ingeo Biopolymer 6201D) supplemented with $6 \%(\mathrm{w} / \mathrm{w})$ sodium stearate to increase electrical polymer conductivity, we were able to produce fibers ranging from 1.00 to $7.00 \mu \mathrm{m}$ in diameter, which are the finest biobased fibers manufactured thus far using our pilot-scale melt-electrospinning device [15]. So far achieved fiber diameters in PLA solution electrospinning are in the range of 0.14 to $2.1 \mu \mathrm{m}$ [16-20] and in melt electrospinning 0.2 to $50 \mu \mathrm{m}[6,12,21-28]$. In individual cases, fiber diameters in the sub-nanometer range have already been achieved using solution electrospinning [29]. However, it should be taken into account that mainly small-scale devices are used and especially in melt electrospinning but also in solution electrospinning additional device modifications such as an integrated airflow are used to achieve fine fiber diameters and a higher process productivity [22, 30].

The fibers using our pilot-scale device were produced without a climate control system, although climate control is common for other device configurations [31-33]. Climate control prevents the rapid cooling of the fibers, so that diameters in the nanometer range can be achieved by drawing before the polymer solidifies. We therefore integrated a climate control system into our pilot-scale melt-electrospinning device by surrounding the spinneret with a glass chamber. We investigated the influence of climate control on the temperature distribution below the spinneret, the melt viscosity of three different PLA grades with increasing melt flow indices (MFIs), and ultimately on the diameter of the resulting fibers at different spin pump speeds. We were able to optimize our pilot-scale device in order to produce fibers with a diameter below $1 \mu \mathrm{m}$ for the first time, thus offering an industrial solution for the preparation of melt-electrospun nanofibers. 


\section{Experimental}

\section{Materials}

Melt electrospinning was carried out using three granular, thermoplastic PLA fiber-grade resins: Ingeo Biopolymer 6201D, 6260D and 6252D (NatureWorks LLC, Minnetonka, Minnesota, USA). The materials are characterized by different MFIs and crystalline melt temperatures. The standard fiber-spinning grade (Ingeo Biopolymer 6201D) has a MFI of $15-30 \mathrm{~g} / 10 \mathrm{~min}$ at $210^{\circ} \mathrm{C}$ and a crystalline melt temperature of $155-170{ }^{\circ} \mathrm{C}$. The first melt-blown grade (Ingeo Biopolymer 6260D) has a MFI of $65 \mathrm{~g} / 10 \mathrm{~min}$ at $210^{\circ} \mathrm{C}$ and a crystalline melt temperature of $165-185^{\circ} \mathrm{C}$. The second melt-blown grade (Ingeo Biopolymer 6252D) has a MFI of $70-85 \mathrm{~g} / 10 \mathrm{~min}$ at $210^{\circ} \mathrm{C}$ and a crystalline melt temperature of $155-170{ }^{\circ} \mathrm{C}$. All materials were vacuum dried at $60^{\circ} \mathrm{C}$ for $12 \mathrm{~h}$ before processing.

\section{Melt-electrospinning equipment}

We used our prototype pilot-scale melt-electrospinning device including a spinneret with 600 nozzles, each $0.3 \mathrm{~mm}$ in diameter and spaced at 8-mm intervals. We integrated a glass chamber around the spinneret to achieve climate control. A schematic illustration (a) and picture (b) of the device including the glass chamber is provided in Figure 5.1. 
a

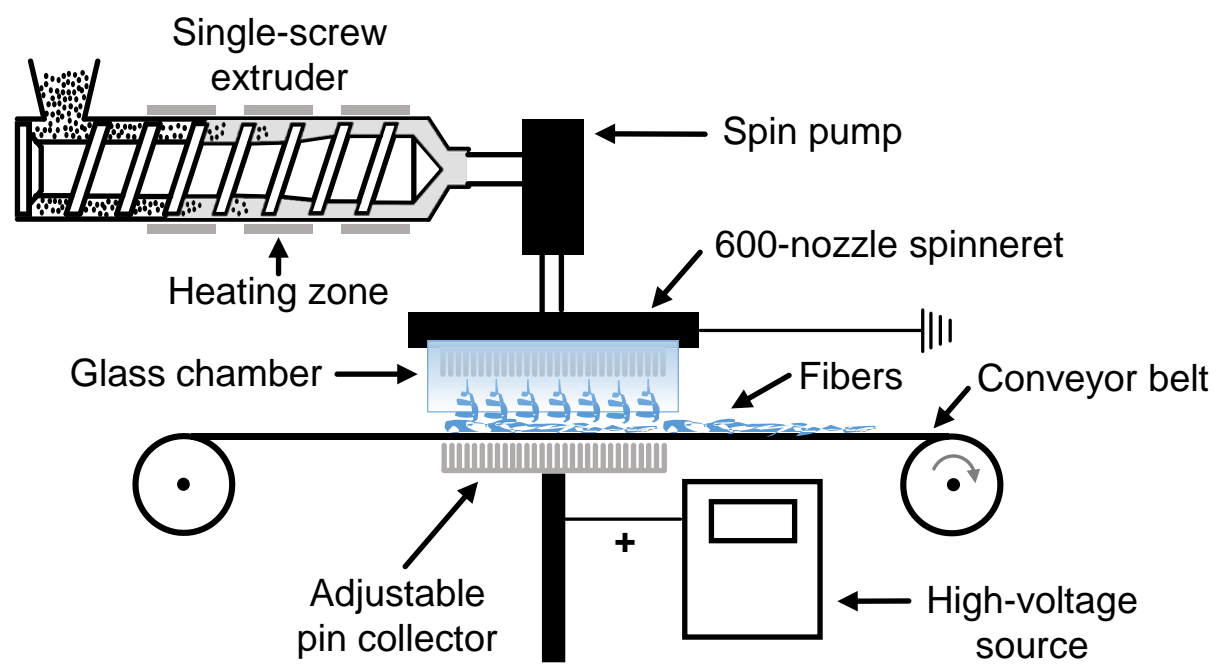

b

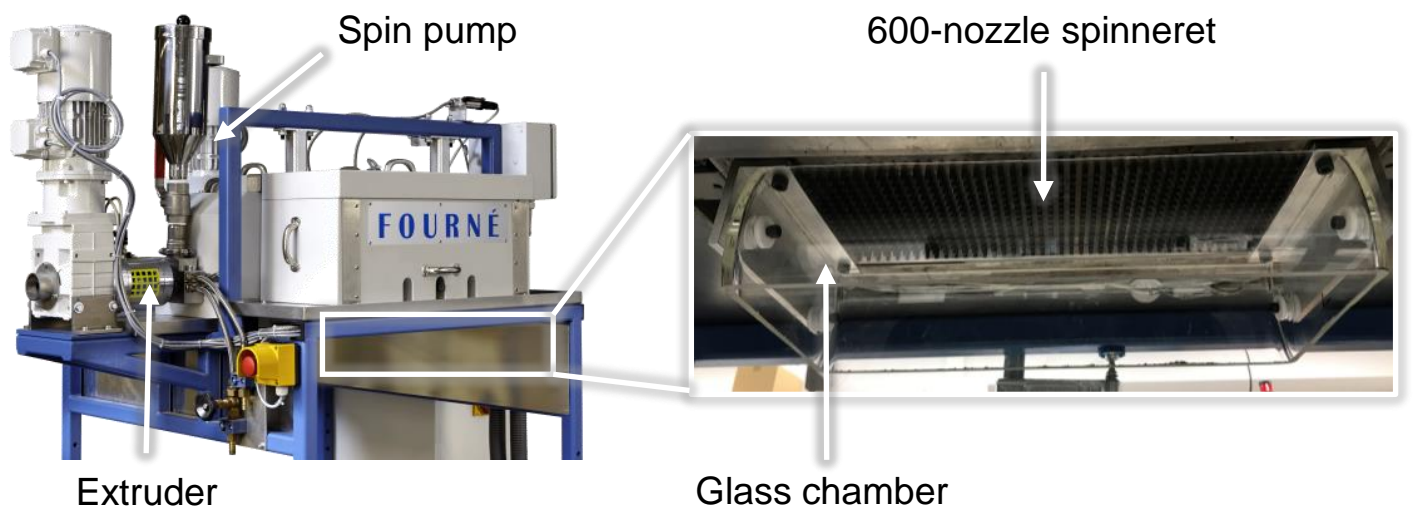

Fig. 5.1: Schematic illustration (a) and picture (b) of our pilot-scale meltelectrospinning device including the glass chamber.

A constant supply of polymer melt was ensured by a speed-adjustable single-screw extruder with three heating zones based on integrated heating elements and a speedcontrolled spinning pump. Spinning was carried out at an adjusted nozzle temperature of $230^{\circ} \mathrm{C}$ without the glass chamber, and at $220^{\circ} \mathrm{C}$ and $230^{\circ} \mathrm{C}$ with the integrated glass chamber using spin pump speeds of 10,5 and $2 \mathrm{rpm}$. Preliminary experiments have shown that at $220-230^{\circ} \mathrm{C}$ a stable fiber formation is ensured over all 600 nozzles and no droplet formation occurs. The statistical relevance of the independent and in this study investigated factors spin pump speed and temperature on the fiber diameter was previously proven with a two-way analysis of variance [34]. An aluminum collector with an uneven surface was used instead of a conventional plate collector. The adjustable collector pins can be placed $8 \mathrm{~mm}$ apart, and we used a narrow and offset pin order with a diagonal distance of $2.6 \mathrm{~cm}$. With the nozzle/collector pairing installed, nonwovens up to $340 \mathrm{~mm}$ in width could be produced on a continuous basis. The distance between the 
collector and nozzle plate was maintained at $11 \mathrm{~cm}$ to allow comparison with our previous results. A positive voltage of $60 \mathrm{kV}$ was applied to the collector with simultaneous grounding of the spinneret.

\section{Polymer characterization}

The rheological properties of the materials, focusing on the frequency-dependent complex viscosity $\eta^{*}$, were characterized using a Discovery HR1 hybrid rheometer (TA Instruments, New Castle, USA). We carried out two frequency sweeps, the first from 628 to $10 \mathrm{rad} / \mathrm{s}$ and the second from 100 to $0.1 \mathrm{rad} / \mathrm{s}$. For all experiments, we used a $25-\mathrm{mm}$ plate-to-plate geometry. The gap distance was set to $1000 \mu \mathrm{m}$, and the strain amplitude was maintained at $1 \%$. Measurements were taken at environmental temperatures of 200 , 230 and $250^{\circ} \mathrm{C}$. To improve the comparability of the results, the viscosity of the different PLA grades is presented at the same frequency of $10 \mathrm{rad} / \mathrm{s}$.

Gel permeation chromatography (GPC), using a 1260 Infinity GPC/SEC System (Aligent Technologies, Santa Clara, USA) with hexafluor-2-isopropanol containing $0.19 \%$ sodium trifluoroacetate as the mobile phase flowing at a rate of $0.33 \mathrm{ml} / \mathrm{min}$, was carried out to determine the relative molecular weight $\left(M_{w}\right)$ and number average molar mass $\left(M_{n}\right)$. The polymer sample $(5 \mathrm{mg}$ ) was dissolved in hexafluor-2-isopropanol for $2 \mathrm{~h}$ before injection into a medium column with $7 \mu \mathrm{m}$ particle size (Polymer Standards Service, Mainz, Germany). The experiment was calibrated against a standard polymethyl methacrylate polymer $\left(1.0 \times 10^{5} \mathrm{~g} / \mathrm{mol}\right)$.

\section{Fiber characterization}

Fiber diameters were measured using a DM4000M reflected light microscope (Leica Microsystems, Wetzlar, Germany) and by scanning electron microscopy (SEM) using a LEO 1450VP instrument (Carl Zeiss, Oberkochen, Germany). The samples were attached to a stub with double-sided carbon tape and sputtered with gold prior to SEM to produce a conductive surface. In each case, the fiber diameter was measured one hundred times at different sections in the nonwoven sample.

\section{Results and discussion}

\section{Effects on viscosity}

The complex viscosity curves of Ingeo Biopolymer 6201D, 6260D and 6252D are compared in Figure 5.2, corresponding to a frequency of $10 \mathrm{rad} / \mathrm{s}$ and set temperatures of 200,230 and $250^{\circ} \mathrm{C}$. There was little difference in viscosity between the two meltblow grades (Ingeo Biopolymer 6260D and 6252D) but the viscosity of the standard spinning-grade PLA (Ingeo Biopolymer 6201D) was approximately three-fold higher at all set temperatures. 


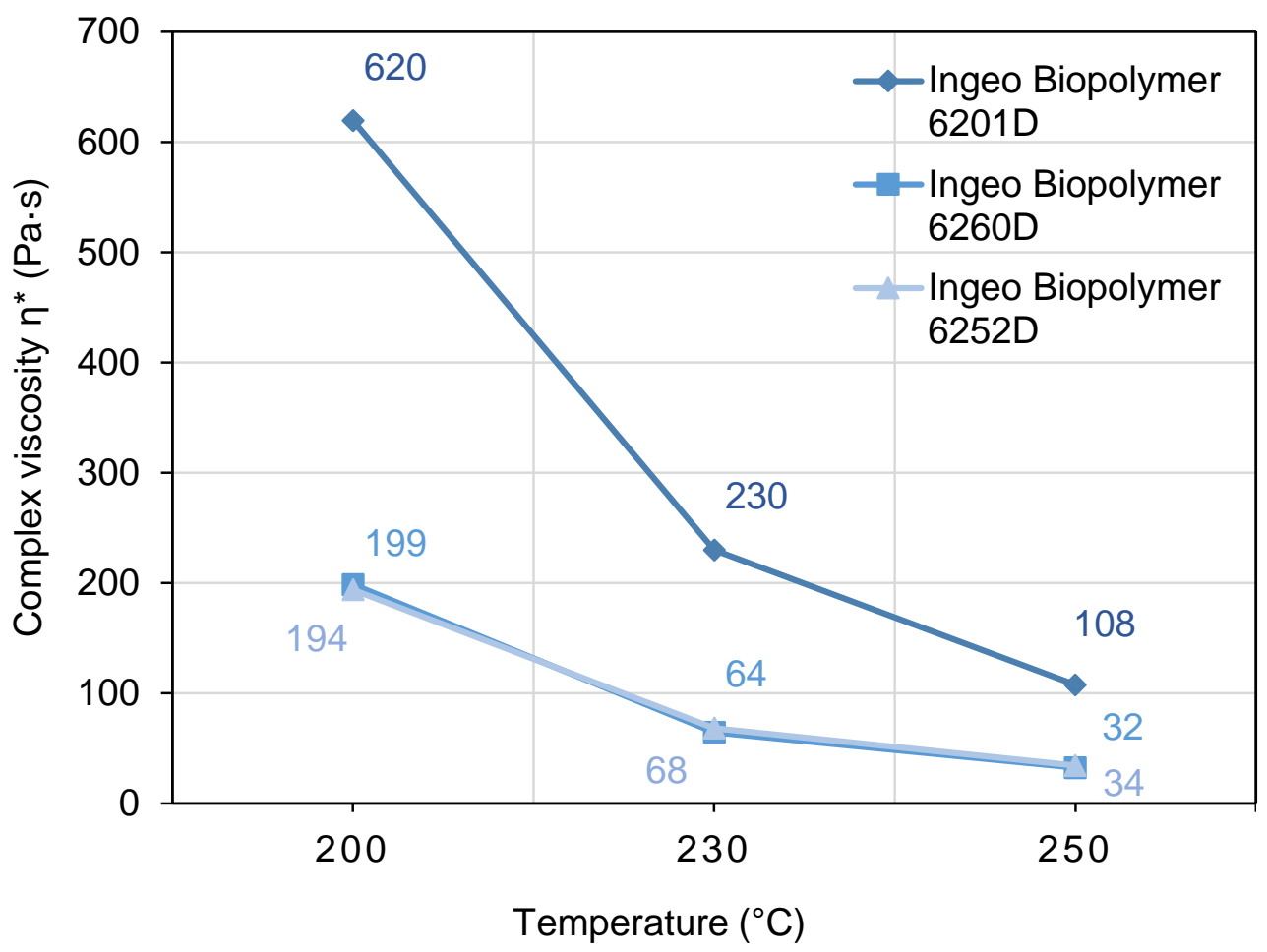

Fig. 5.2: Complex viscosity of Ingeo Biopolymer 6201, 6260 and 6252 determined at a frequency of $10 \mathrm{rad} / \mathrm{s}$ at set temperatures of 200,230 and $250{ }^{\circ} \mathrm{C}$.

Table 5.1 presents the relative molecular weight $\left(\mathrm{M}_{\mathrm{w}}\right)$ and number average molar mass $\left(\mathrm{M}_{\mathrm{n}}\right)$ of Ingeo Biopolymer 6201, 6260 and 6252. The decrease in molecular weight correlates with the increasing MFI values of the individual grades given by the polymer manufacturer.

Tab. 5.1: Relative molecular weight $\left(M_{w}\right)$ and number average molar mass $\left(M_{n}\right)$ of Ingeo Biopolymer 6201, 6260 and 6252.

\begin{tabular}{|l|l|l|}
\hline Material & $\begin{array}{l}\mathbf{M}_{\mathbf{w}} \\
(\mathbf{g} / \mathbf{m o l})\end{array}$ & $\begin{array}{l}\mathbf{M}_{\mathbf{n}} \\
(\mathbf{g} / \mathbf{m o l})\end{array}$ \\
\hline Ingeo Biopolymer 6201 & 147700 & 86170 \\
\hline Ingeo Biopolymer 6260 & 117500 & 72540 \\
\hline Ingeo Biopolymer 6252 & 103400 & 66730 \\
\hline
\end{tabular}

\section{Temperature distribution}

At a set spinneret temperature of $230^{\circ} \mathrm{C}$, the average surface temperature of the spinneret was $112^{\circ} \mathrm{C}$ after 2 hours of operation and $117^{\circ} \mathrm{C}$ after 4 hours of operation without the glass chamber, as determined using an infrared thermometer. The polymer inlet was one-sided, so temperature differences of up to $30^{\circ} \mathrm{C}$ were detected over the 
spinneret surface [31]. Table 5.2 shows the temperature profiles at spinning temperatures of 220 and $230{ }^{\circ} \mathrm{C}$ using the glass chamber.

The heat accumulation within the chamber caused the surface temperature of the spinneret to increase by $30^{\circ} \mathrm{C}$ at a set temperature of $220^{\circ} \mathrm{C}$ and by $40^{\circ} \mathrm{C}$ at $230^{\circ} \mathrm{C}$. As the distance to the spinneret increased, the temperature slowly declined until the temperature at the edge of the glass chamber reached 38 and $49^{\circ} \mathrm{C}$ at set temperatures of 220 and $230^{\circ} \mathrm{C}$, respectively. This means that both temperature profiles fell below the glass transition temperature of the three PLA grades we used $\left(55-60^{\circ} \mathrm{C}\right)$, thus avoiding the sticking and deformation of the fibers during deposition on the conveyor belt. Furthermore, the temperature remained above the glass transition temperature up to a distance of $4 \mathrm{~cm}$ from the spinneret, which allows further stretching of the fibers.

Tab. 5.2: Temperature profiles at spinning temperatures of 220 and $230{ }^{\circ} \mathrm{C}$ with the glass chamber installed. .

\begin{tabular}{|l|l|l|}
\hline $\begin{array}{l}\text { Distance from spinneret } \\
\text { (cm) }\end{array}$ & \multicolumn{2}{|c|}{$\begin{array}{r}\text { Temperature below spinneret }\left({ }^{\circ} \mathbf{C}\right) \text { at set } \\
\text { spinneret temperatures of: }\end{array}$} \\
\cline { 2 - 3 } & $\mathbf{2 2 0}^{\circ} \mathbf{C}$ & $\mathbf{2 3 0}{ }^{\circ} \mathbf{C}$ \\
\hline 0 & 144 & 158 \\
\hline 2 & 89 & 103 \\
\hline 3 & 74 & 88 \\
\hline 4 & 62 & 73 \\
\hline 5 & 55 & 62 \\
\hline 6 & 47 & 56 \\
\hline Outer edge & 38 & 49 \\
\hline
\end{tabular}

\section{Fiber diameters}

Next we investigated the processability of the three PLA grades and the influence of the temperature profile induced by installing the glass chamber on the fiber diameter. Table 5.3 shows the average fiber diameters ( \pm standard deviations) produced at a set spinneret temperature of $230{ }^{\circ} \mathrm{C}$ without the glass chamber, and at 220 and $230{ }^{\circ} \mathrm{C}$ with the glass chamber, at three different spin pump speeds. 
Tab. 5.3: Average fiber diameters ( \pm standard deviations) produced at $230{ }^{\circ} \mathrm{C}$ without the glass chamber (GC), and at 220 and $230{ }^{\circ} \mathrm{C}$ with the glass chamber installed, at spin pump speeds of 10,5 and $2 \mathrm{rpm}$.

Material

Fiber diameter $(\mu \mathrm{m})$ at spin pump speeds of:

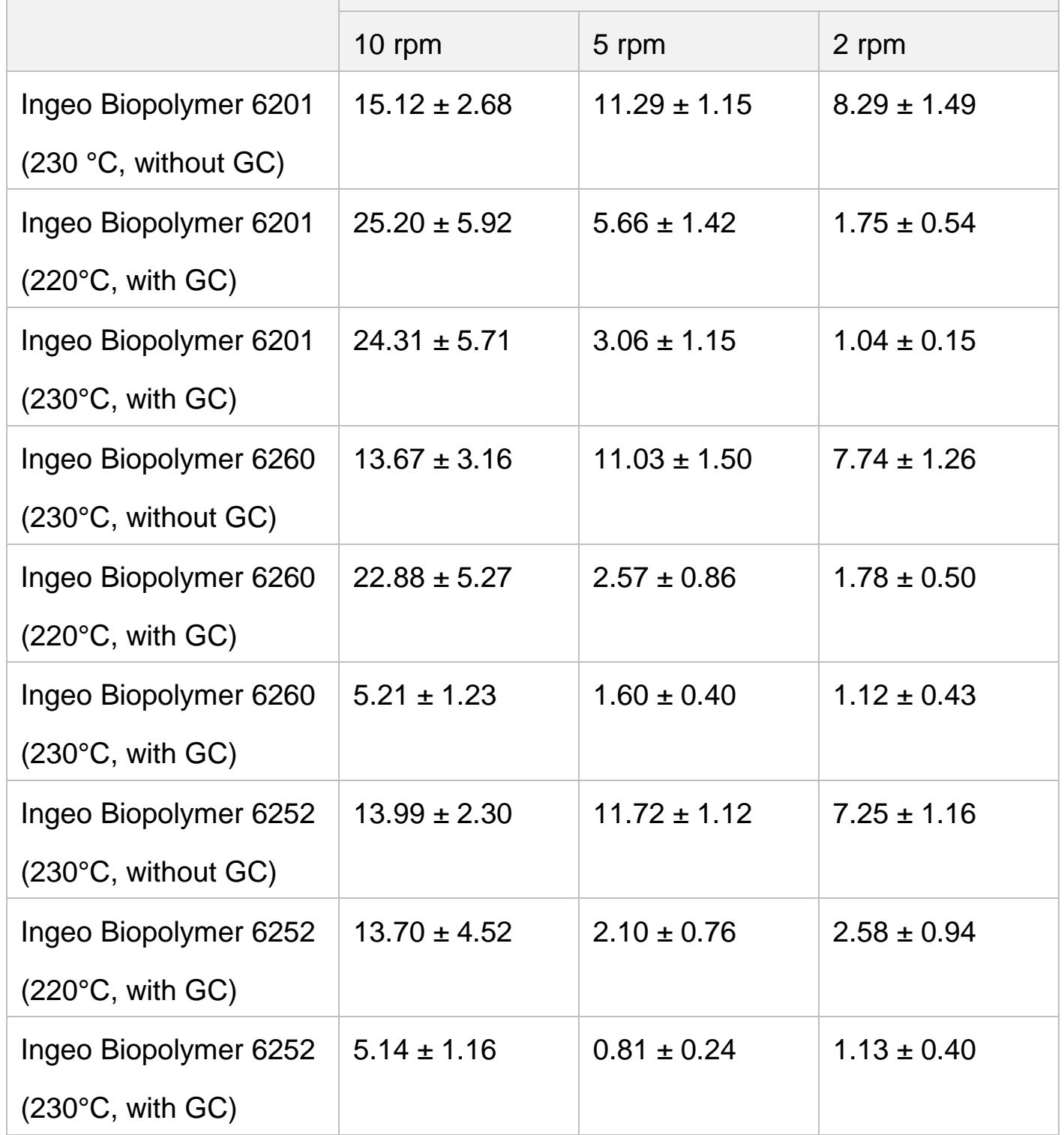

Comparing the diameter of fibers produced from Ingeo Biopolymer 6201 (Figure 5.3), Ingeo Biopolymer 6260 (Figure 5.4), and Ingeo Biopolymer 6252 (Figure 5.5), revealed no significant difference between the PLA grades at a set spinneret temperature of $230{ }^{\circ} \mathrm{C}$ without the glass chamber, but a $50 \%$ reduction as the pump speed was reduced from $10 \mathrm{rpm}(\sim 15 \mu \mathrm{m})$ to $2 \mathrm{rpm}(\sim 7 \mu \mathrm{m})$. 


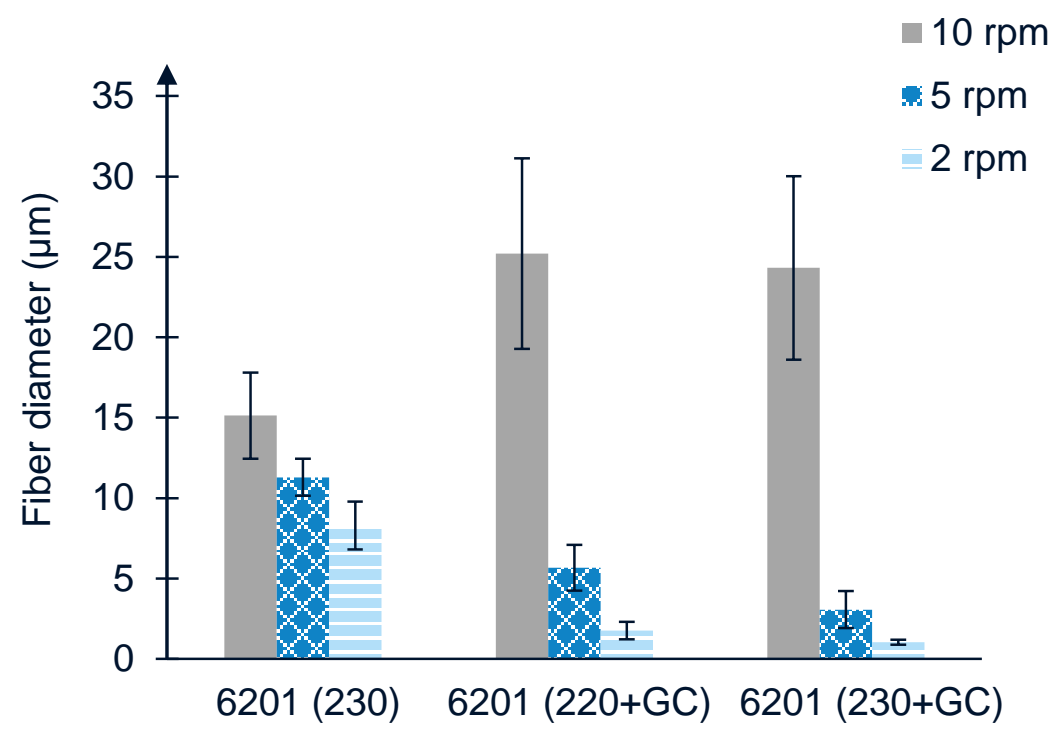

Fig. 5.3: Diameters of Ingeo Biopolymer 6201 fibers produced at $230{ }^{\circ} \mathrm{C}$ without the glass chamber (GC), and at 220 and $230{ }^{\circ} \mathrm{C}$ with the glass chamber installed, at spin pump speeds of 10,5 and $2 \mathrm{rpm}$.

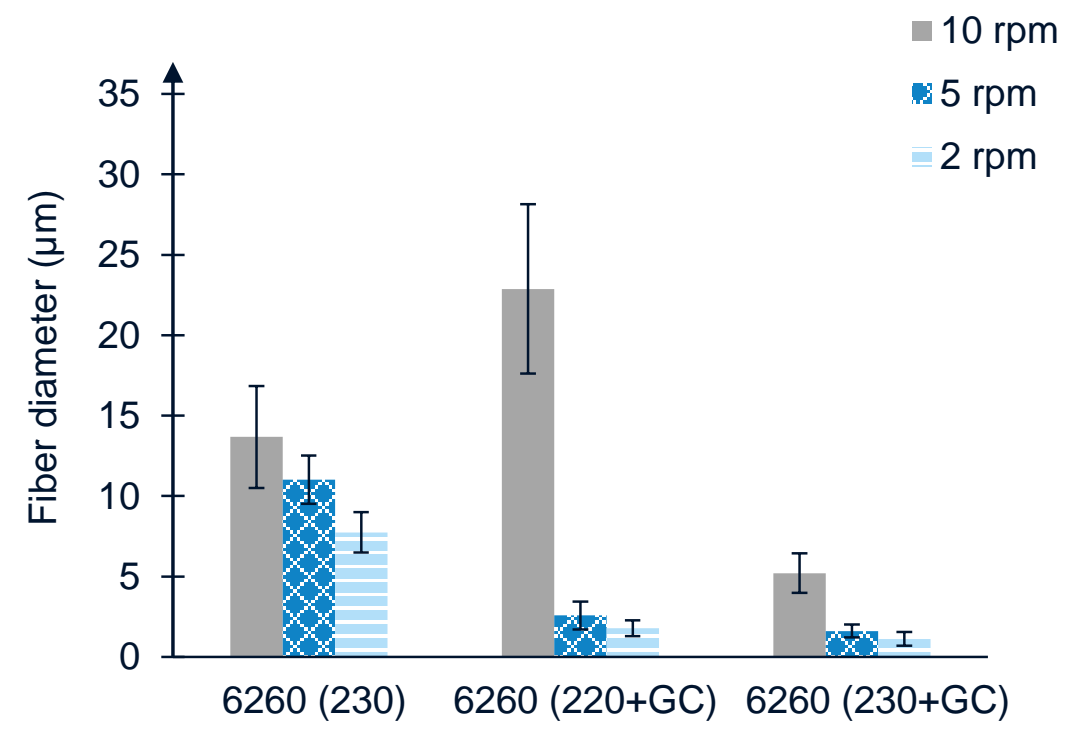

Fig. 5.4: Diameters of Ingeo Biopolymer 6260 fibers produced at $230^{\circ} \mathrm{C}$ without the glass chamber (GC), and at 220 and $230^{\circ} \mathrm{C}$ with the glass chamber installed, at spin pump speeds of 10,5 and $2 \mathrm{rpm}$. 


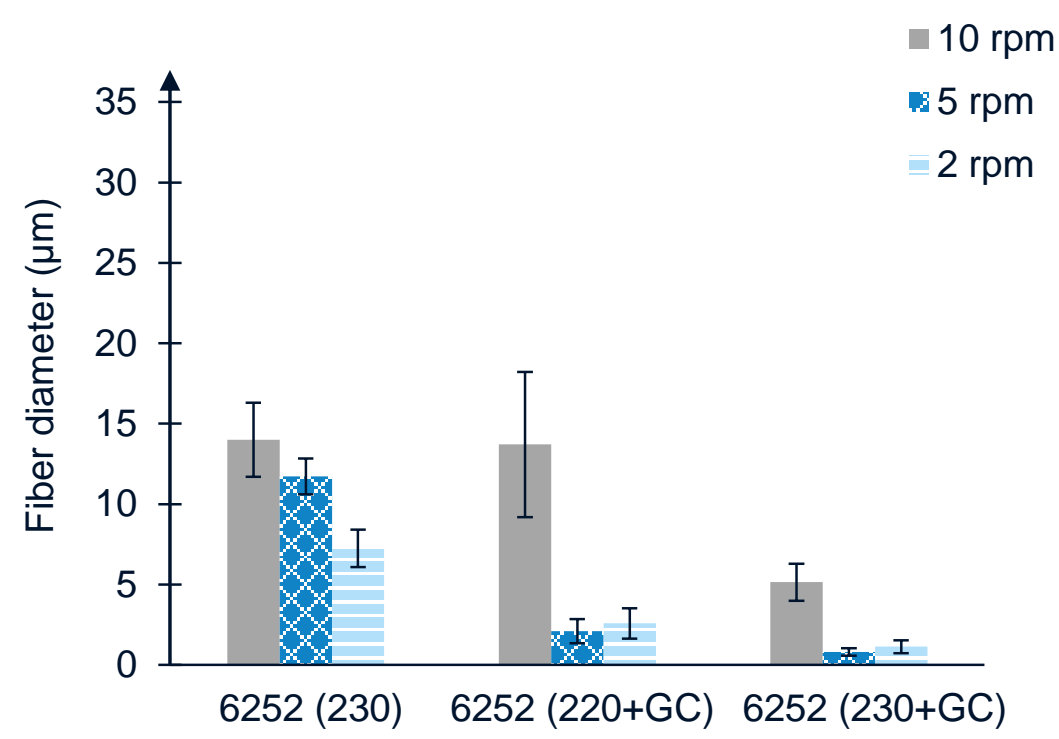

Fig. 5.5: Diameters of Ingeo Biopolymer 6252 fibers produced at $230^{\circ} \mathrm{C}$ without the glass chamber (GC), and at 220 and $230{ }^{\circ} \mathrm{C}$ with the glass chamber installed, at spin pump speeds of 10,5 and $2 \mathrm{rpm}$.

Figure 5.6 shows the microscope images of the melt-electrospun fibers at a set temperature of $230^{\circ} \mathrm{C}$ without the glass chamber at a spin pump speed of 10 and $2 \mathrm{rpm}$; (a) Ingeo Biopolymer 6201 at 10 rpm, (b) at 2 rpm; (c) Ingeo Biopolymer 6260 at $10 \mathrm{rpm}$, (d) at 2 rpm; and (e) Ingeo Biopolymer 6252 at 10 rpm, (f) at 2 rpm. 

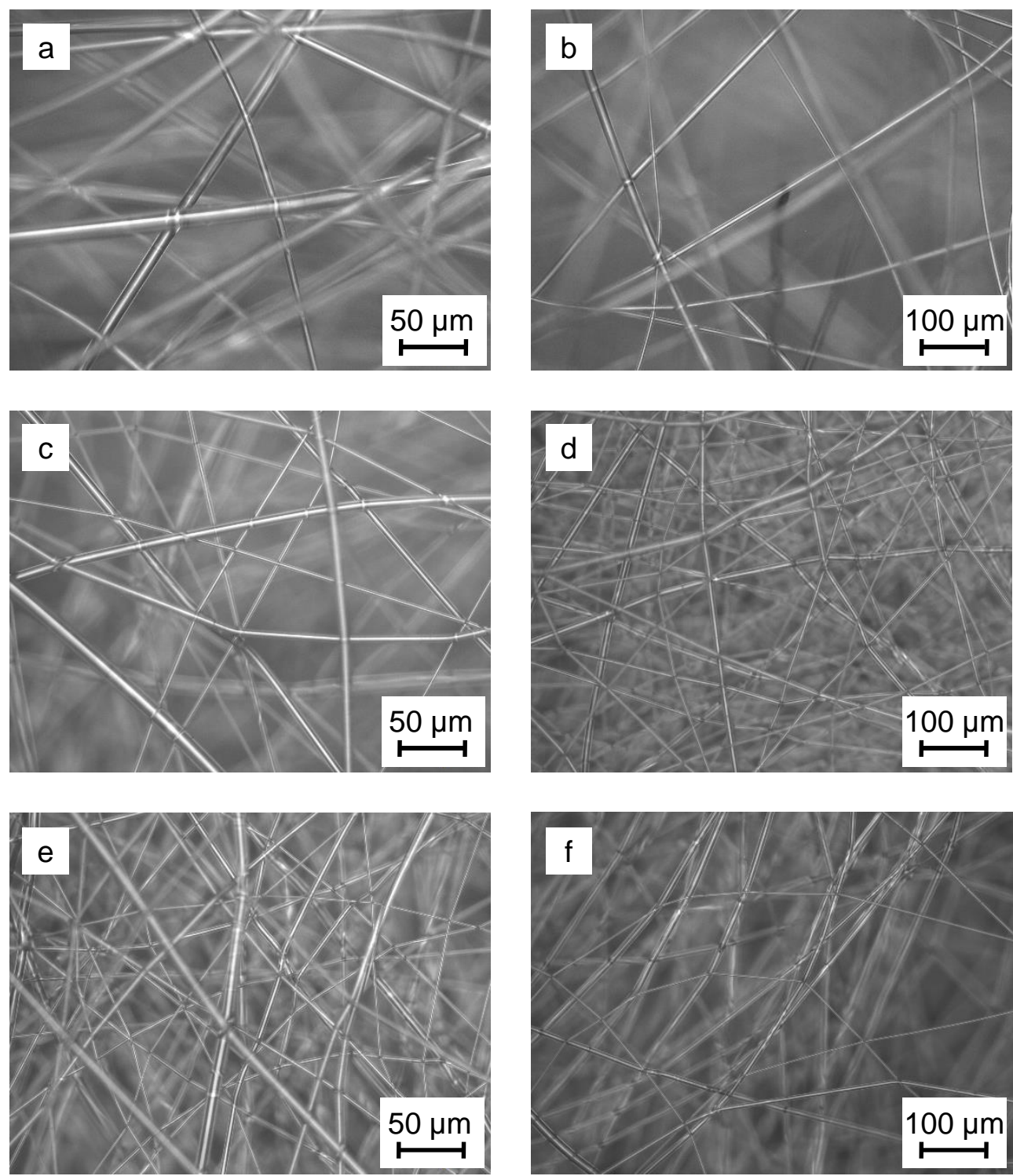

Fig. 5.6: Microscope images of the melt-electrospun fibers at a set temperature of $230^{\circ} \mathrm{C}$ without the glass chamber at a spin pump speed of 10 and $2 \mathrm{rpm}$; (a) Ingeo Biopolymer 6201 at 10 rpm, (b) at 2 rpm; (c) Ingeo Biopolymer 6260 at 10 rpm, (d) at 2 rpm; and (e) Ingeo Biopolymer 6252 at 10 rpm, (f) at $2 \mathrm{rpm}$.

Finer fibers were anticipated at lower spin pump speeds and melt flow rates, as observed during previous studies [3, 32, 33]. Exemplary, Figure 5.7 shows the nonwoven of Ingeo Biopolymer 6260 melt-electrospun without the glass chamber at a set temperature of $230^{\circ} \mathrm{C}$ and a spin pump speed of (a) $10 \mathrm{rpm}$ and (b) $2 \mathrm{rpm}$. A droplet formation could not be observed with or without the glass chamber. 

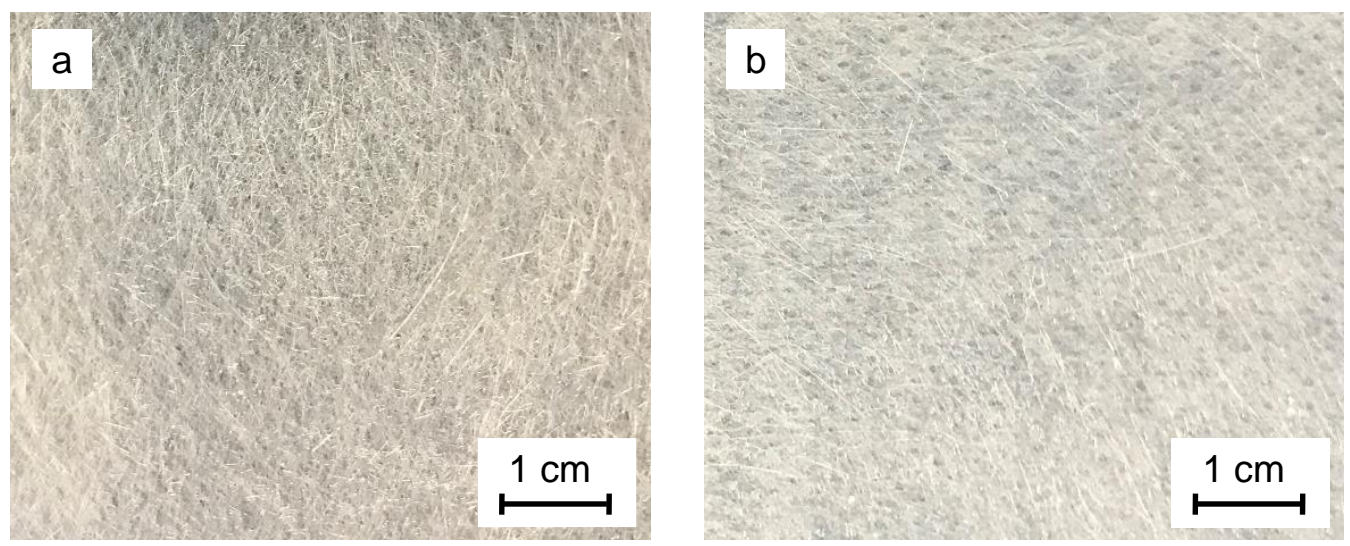

Fig. 5.7: Nonwoven of Ingeo Biopolymer 6260 melt-electrospun without the glass chamber at a set temperature of $230^{\circ} \mathrm{C}$ and a spin pump speed of (a) $10 \mathrm{rpm}$ and (b) $2 \mathrm{rpm}$.

The nonwovens were deposited on a blue cellulose substrate. At lower spin pump speeds, the PLA fibers are attracted more strongly by the collector pins at which the electric field lines emerge, resulting in slight accumulations that leave a visible structure in the PLA nonwoven. To overcome this phenomenon and improve the overlap, the distance between the pins can be reduced in future studies.

The differing viscosities of the three materials appeared to have no effect. Contrary to expectations, the diameter of Ingeo Biopolymer 6201 fibers increased significantly at a pump speed of $10 \mathrm{rpm}$ and at set temperatures of 220 and $230^{\circ} \mathrm{C}$ following the integration of the glass chamber, possibly because the higher melt flow rate resulting from the lower viscosity does not give sufficient time for drawing. The same phenomenon was observed for Ingeo Biopolymer 6260 at a set temperature of $220^{\circ} \mathrm{C}$. However, the diameter of Ingeo Biopolymer 6252 fibers at $220^{\circ} \mathrm{C}$ remained almost the same with or without the glass chamber. Nevertheless, the diameter of Ingeo Biopolymer 6260 and 6252 fibers could be reduced by more than $50 \%$ at a spinning temperature of $230{ }^{\circ} \mathrm{C}$ and a spin pump speed of $10 \mathrm{rpm}$ by installing the glass chamber. Otherwise, the fiber diameter declined with slower spin pump speeds and the lowest average fiber diameter of $1.04 \mu \mathrm{m}$ was achieved for Ingeo Biopolymer 6201 at a spinning temperature of $230{ }^{\circ} \mathrm{C}$ with the glass chamber installed and a spin pump speed of $2 \mathrm{rpm}$. For the first time, individual fibers with diameters of less than $1 \mu \mathrm{m}$ were observed, improving on our previous results for PLA supplemented with $6 \%(\mathrm{w} / \mathrm{w})$ sodium stearate, which generated individual fibers with a minimum diameter of $1.23 \mu \mathrm{m}$ [15].

For all three PLA grades, we achieved the lowest fiber diameters at a spinning temperature of $230^{\circ} \mathrm{C}$ with the glass chamber installed. The diameter of Ingeo Biopolymer 6260 fibers could be reduced by $86 \%$ at a spin pump speed of $2 \mathrm{rpm}$ compared to spinning in the absence of the glass chamber. In this case, we also observed individual sub-micrometer fibers. The minimum average fiber diameter was $0.81 \mu \mathrm{m}$, which was achieved using Ingeo Biopolymer 6252, a spinneret temperature of $230^{\circ} \mathrm{C}$, a spin pump speed of $5 \mathrm{rpm}$, and the glass chamber installed (Figure 5.8). 

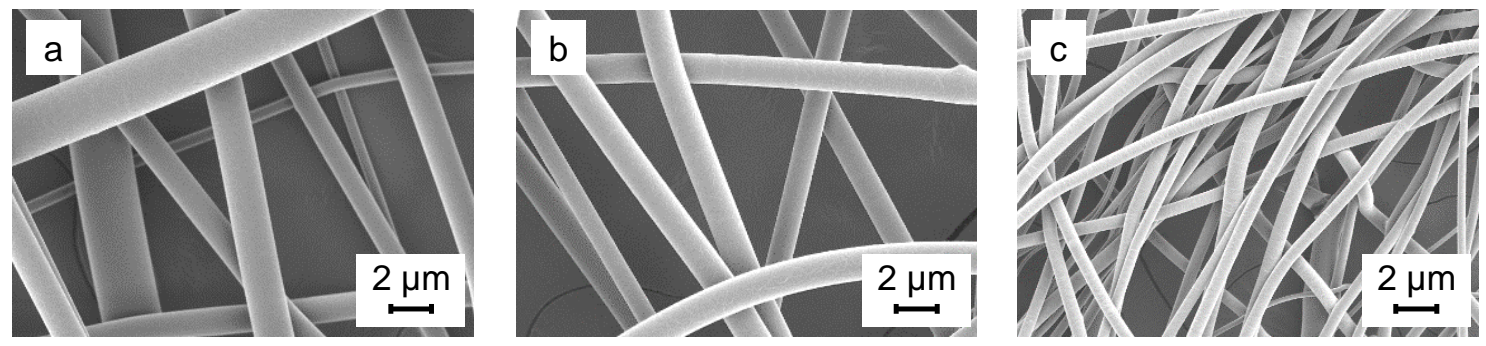

Fig. 5.8: SEM image of (a) Ingeo Biopolymer 6201, (b) Ingeo Biopolymer 6260 and (c) Ingeo Biopolymer 6252 fibers at a set temperature of $230{ }^{\circ} \mathrm{C}$ and a spin pump speed of $5 \mathrm{rpm}$ using the glass chamber.

The finest individual fiber was $0.42 \mu \mathrm{m}$ in diameter. We were therefore able to achieve the production of nanofibers as fine as any previously achieved by solution or melt electrospinning on a device which comes closer to industrial-scale melt electrospinning than any described in the literature before. The scalability of our process therefore brings industrial melt electrospinning one step closer. The installment of the glass chamber did not appear to affect the electric field during spinning, although this should be examined more closely in future experiments.

\section{Conclusion}

We have previously reported the use of our prototype melt-electrospinning device for the production of biobased thermoplastic sub-microfibers and nanofibers. The finest PLA fibers we were able to produce were $\sim 1 \mu \mathrm{m}$ in diameter, but this was achieved without comprehensive optimization of the apparatus, the process parameters, or the polymer substrate. Now we have integrated a climate control system realized as a glass chamber, which generated a temperature gradient to ensure efficient heating and therefore prolonged the drawing of the fibers. We tested three different PLA grades and produced individual fibers with diameters in the nanometer range when the glass chamber was integrated, although the average fiber diameters remained predominantly above the threshold of $1 \mu \mathrm{m}$. Nevertheless, an average fiber diameter of $0.81 \mu \mathrm{m}$ was achieved with polylactic acid Ingeo Biopolymer 6252, and the finest individual fiber $(0.42 \mu \mathrm{m}$ in diameter) was produced from this polymer at a spin pump speed of $5 \mathrm{rpm}$ and a spinneret set temperature of $230^{\circ} \mathrm{C}$. In contrast to other systems, our device does not require (hot) compressed air or additional heating of the glass chamber. Furthermore, no additives, as usual in melt electrospinning, were used, which enables easier approval for pharmaceutical and biomedical products. We have therefore demonstrated the innovative performance of our pilot-scale melt-electrospinning device, which bridges the gap between laboratory-scale and pilot-scale manufacturing and achieves fiber diameters comparable to those produced by conventional solution electrospinning. 


\section{Acknowledgments}

The authors acknowledge support from the Microscopy Department, Institute for Textile Technology, RWTH Aachen University, Germany; Fourné Maschinenbau GmbH, AlfterImpekoven, Germany; Pötter-Klima Gesellschaft für Nanoheiztechnik mbH, Georgsmarienhütte, Germany; and Schnick Systemtechnik GmbH \& Co. KG, Heiligenhaus, Germany. 


\section{References}

[1] Greiner A, Wendorff JH. Electrospinning: A Fascinating Method for the Preparation of Ultrathin Fibers. Angewandte Chemie International Edition. 2007; 46(30):5670703.

[2] Jiang S, Chen Y, Duan G, Mei C, Greiner A, Agarwal S. Electrospun nanofiber reinforced composites: a review. Polymer Chemistry. 2018; 9(20):2685-720.

[3] Brown T, Dalton P, Hutmacher DW. Melt electrospinning today: an opportune time for an emerging polymer process. Elsevier. 2016; 56.

[4] Bhardwaj N, Kundu SC. Electrospinning: a fascinating fiber fabrication technique. Biotechnol Adv. 2010; 28.

[5] Ramakrishna S, Fujihara K, Teo W-E. Electrospun nanofibers: solving global issues. Mater Today. 2006;9.

[6] Qin Y, Cheng L, Zhang Y, Chen X, Wang X, He X, et al. Efficient preparation of poly(lactic acid) nanofibers by melt differential electrospinning with addition of acetyl tributyl citrate. Journal of Applied Polymer Science. 2018; 135(31):46554.

[7] Nayak R. Polypropylene nanofibers: melt electrospinning versus Meltblowing. Engineering Material: Springer International Publishing; 2017.

[8] Chen M, Zhang Y, Chen X, Yang W, Li H, Yousefzadeh M, et al. Polymer melt differential electrospinning from a linear slot spinneret. Journal of Applied Polymer Science:48922.

[9] Bubakir M, Li H, Yang W. Advances in Melt Electrospinning Technique. 2017. p. $1-30$.

[10] Zhao F, Liu Y, Yuan H. Orthogonal design study on factors affecting the degradation of polylactic acid fibers of melt electrospinning. J Appl Polym Sci. $2012 ; 125$.

[11] $\mathrm{Li} \mathrm{H}$, Chen $H$, Zhong $X$. Interjet distance in needleless melt differential electrospinning with umbellate nozzles. J Appl Polym Sci. 2014; 131.

[12] Liu Y, Zhao F, Zhang C, Zhang J, Yang W. Solvent-free preparation of poly(lactic acid) fibers by melt electrospinning using an umbrella-like spray head and alleviation of the problematic thermal degradation. J Serb Chem Soc. 2012; 77.

[13] Hacker C. Anlagenentwicklung für das Elektroschmelzspinnen von Feinfaservliesstoffen für die Abwasseraufbereitung [Doctoral thesis]. Shaker: RWTH Aachen University; 2015.

[14] Hacker C, Seide G, Gries T, Thomas H, Moeller M, editors. Electrospinning of polymer melt: steps toward an upscaled multi-jet process. International Conference latest advances in high tech textiles and textile-based materials; 2009. 
[15] Koenig K, Beukenberg K, Langensiepen F, Seide G. A new prototype meltelectrospinning device for the production of biobased thermoplastic submicrofibers and nanofibers. Biomaterials Research. 2019; 23(1):10.

[16] Tsuji H, Nakano M, Hashimoto M, Takashima K, Katsura S, Mizuno A. Electrospinning of Poly(lactic acid) Stereocomplex Nanofibers. Biomacromolecules. 2006; 7(12):3316-20.

[17] Badami AS, Kreke MR, Thompson MS, Riffle JS, Goldstein AS. Effect of fiber diameter on spreading, proliferation, and differentiation of osteoblastic cells on electrospun poly(lactic acid) substrates. Biomaterials. 2006; 27(4):596-606.

[18] Kayaci F, Umu OCO, Tekinay T, Uyar T. Antibacterial Electrospun Poly(lactic acid) (PLA) Nanofibrous Webs Incorporating Triclosan/Cyclodextrin Inclusion Complexes. Journal of Agricultural and Food Chemistry. 2013; 61(16):3901-8.

[19] Casasola R, Thomas NL, Trybala A, Georgiadou S. Electrospun poly lactic acid (PLA) fibres: Effect of different solvent systems on fibre morphology and diameter. Polymer. 2014; 55(18):4728-37.

[20] Jahanmardi Y, Tavanaie MA, Tehrani-Bagha AR. Curcumin release from blended polycaprolactone/polylactic acid electrospun nanofibrous meshes. Journal of Industrial Textiles.0(0):1528083719851845.

[21] Zhou H, Green TB, Joo YL. The thermal effects on electrospinning of polylactic acid melts. Polymer (Guildf). 2006; 47.

[22] Zhmayev E, Cho D, Joo YL. Nanofibers from gas-assisted polymer melt electrospinning. Polymer. 2010; 51(18):4140-4.

[23] Ogata N, Yamaguchi S, Shimada N, Lu G, Iwata T, Nakane K, et al. Poly(lactide) nanofibers produced by a melt-electrospinning system with a laser melting device. Journal of Applied Polymer Science. 2007; 104(3):1640-5.

[24] Carroll CP, Zhmayev E, Kalra V, Joo YL. Nanofibers from electrically driven viscoelastic jets: modeling and experiments. Korea-Australia Rheology Journal. 2008; 20(3):153-64.

[25] Chrzanowska O, Struszczyk M, Krucińska I. Small Diameter Tubular Structure Design Using Solvent-Free Textile Techniques. Journal of Applied Polymer Science. 2014; 131.

[26] Mazalevska O, Struszczyk M, Chrzanowski M, Krucińska I. Application of Electrospinning for Vascular Prothesis Design-Preliminary Results. Fibres and Textiles in Eastern Europe. 2011; 19:46-52.

[27] Mazalevska O, Struszczyk MH, Krucinska I. Design of vascular prostheses by melt electrospinning-structural characterizations. Journal of Applied Polymer Science. 2013; 129(2):779-92. 
[28] Zhmayev E, Zhou H, Joo YL. Modeling of non-isothermal polymer jets in melt electrospinning. Journal of Non-Newtonian Fluid Mechanics. 2008; 153(2):95-108.

[29] Jian S, Zhu J, Jiang S, Chen S, Fang H, Song Y, et al. Nanofibers with diameter below one nanometer from electrospinning. RSC Advances. 2018; 8(9):4794-802.

[30] Duan G, Greiner A. Air-Blowing-Assisted Coaxial Electrospinning toward High Productivity of Core/Sheath and Hollow Fibers. Macromolecular Materials and Engineering. 2019; 304:1800669.

[31] Koenig K, Daenicke J, Langensiepen F, Seide G, Schubert DW. From lab to pilot scale: melt electrospun nanofibers of polypropylene with conductive additives. J Nanomater Mol Nanotechnol. 2019; 8(2).

[32] Nayak R. Effect of viscosity and electrical conductivity on the morphology and fiber diameter in melt electrospinning of polypropylene. Textile Research Journal. 2013; 83(6):606

[33] Nayak R, Kyratzis IL, Truong YB, Padhye R, Arnold L. Melt-electrospinning of polypropylene with conductive additives. Journal of Materials Science. 2012; 47(17):6387-96.

[34] Koenig K, Hermanns S, Ellerkmann J, Saralidze K, Langensiepen F, Seide G. The effect of additives and process parameters on the pilot-scale manufacturing of polylactic acid sub-microfibers by melt electrospinning. Textile Research Journal. 2020:0040517520904019. 


\title{
Chapter 6
}

\author{
Valorization
}

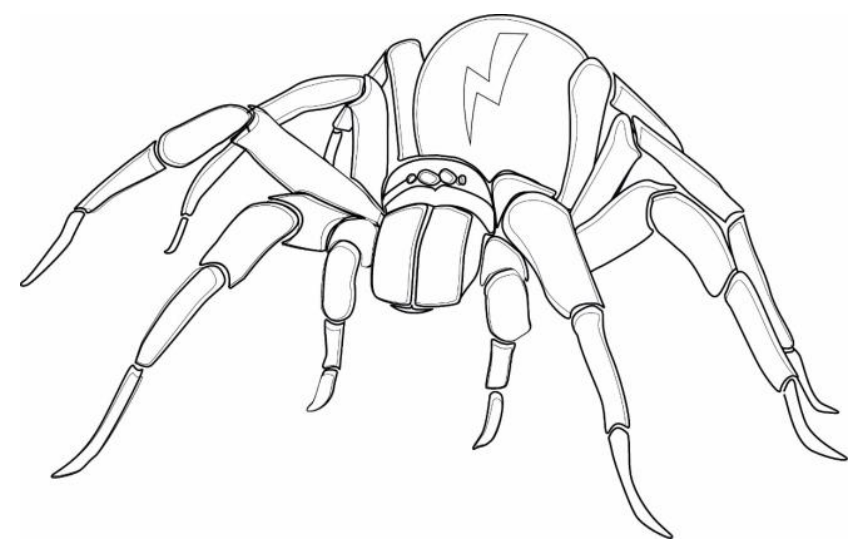




\section{Valorization}

The unique properties of nanofibers, such as high flexibility and an enormous specific surface area, making them suitable for applications in various fields such as medicine, energy and electronics, filtration and separation, and the manufacture of textiles, have significantly increased research interest in efficient nanofiber manufacturing processes [1]. Nevertheless, a key challenge for industry is the ability to upscale nanofiber production. Several methods, as described in Chapter 1, can be used to produce nanofibers and sub-microfibers, but melt electrospinning is among the most promising technologies in terms of fiber structure and the breadth of downstream applications due to the absence of solvents in the manufacturing process [2]. The major drawback of melt electrospinning is its low throughput, resulting in the adoption of solution electrospinning as the prevailing industrial process technology. Recent device developments such as multiple needle and needleless configurations have demonstrated a roadmap to overcome the low throughput in melt electrospinning, although an industrial process has yet to be established [3]. In this thesis, a new developed scaled-up melt-electrospinning prototype featuring 600 nozzles, bridging the gap between laboratory-scale and industrial-scale nanofiber manufacturing, has been presented. The prototype device vastly exceeds the capabilities of any state-of-the-art technology and has been successfully used to produce nanofibers with the same quality and properties compared to nanofibers produced with the more conventional solution electrospinning process.

In addition, we were able to make a decisive contribution to the still necessary material development for melt-electrospinable polymers [3,4] based on polypropylene (Chapter 3) and the biobased polymer polylactic acid (Chapter 4). With the approach of adding additives such as salts like sodium stearate, oleate and chloride as well as the biobased dyed alizarin, hematoxylin and quercetin in combination with plasticizers [5], we were able to overcome the obstacles of poor polymer conductivity and insufficient viscosity of the initial polymers, thus successfully approaching fiber production in the sub-micro range [2]. For the first time, the correlation between spin pump speed, temperature/viscosity and additive concentration/conductivity on the reduction of the fiber diameter could be statistically validated for a pilot-scale melt-electrospinning device using SPSS two-way analysis of variance [2]. This validation will facilitate further material development, as basic principles such as a decrease in fiber diameter with decreasing viscosity and decreasing spin pump speed, can be assumed to be known. Thus, it is possible to focus on the investigation of the polymer melt conductivity in order to achieve a classification of melt-electrospinable polymers. By introducing the climate control system in the form of a glass chamber (Chapter 5), fiber diameters, which are comparable to those produced by conventional solution electrospinning, could be achieved, thereby emphasizing the innovative performance of the prototype.

For a better comprehension of the fiber diameter dimensions achieved in this thesis, the fiber diameters produced with the pilot-scale melt-electrospinning device have been 
related to the fiber diameters depending on the nanofiber production methods described in Chapter 1 (Figure 6.1).

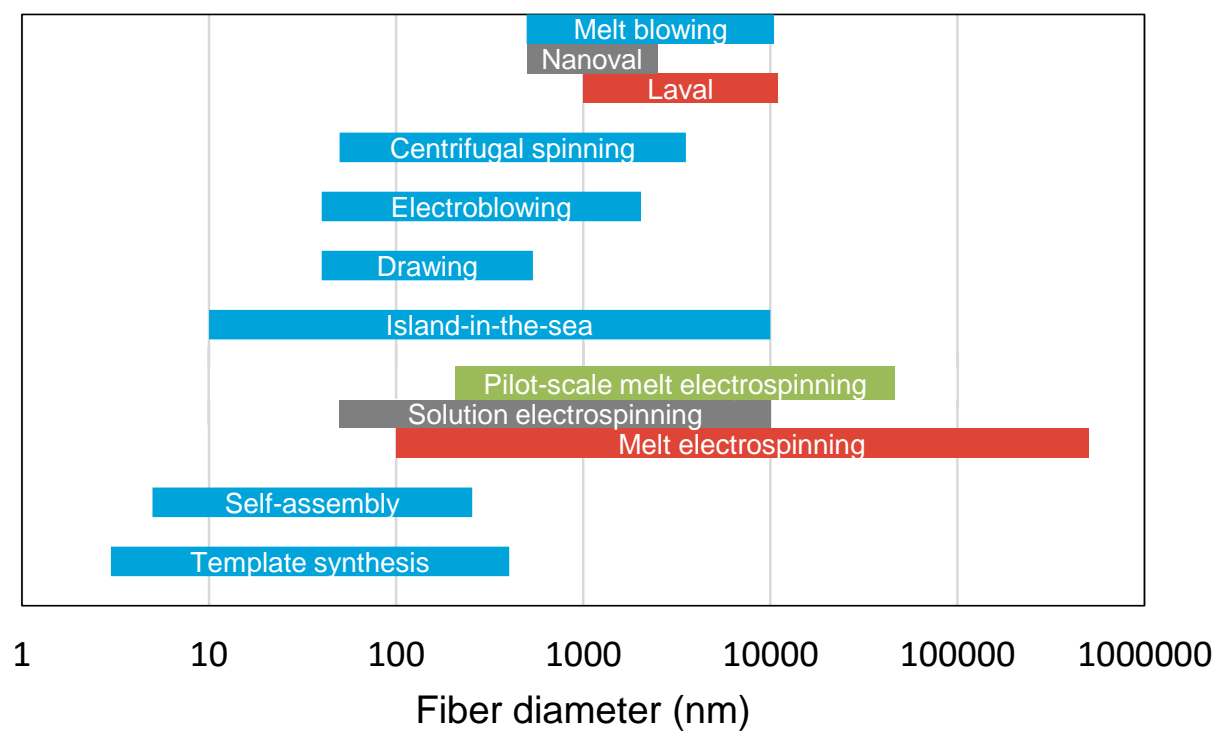

Fig. 6.1: Fiber diameters obtained from pilot-scale melt electrospinning compared to other production methods.

It has to be taken into account that most melt-electrospun sub-microfibres are produced using small devices that are not suitable for sustainable, industrial production $[3,6,7]$. For a multi-nozzle melt-electrospinning device with 600 nozzles, as presented in this thesis, the average fiber diameter of $810 \mathrm{~nm}$ and single fiber diameter of $420 \mathrm{~nm}$ is the smallest fiber diameter achieved so far.

In the following, the utilization of the novel prototype melt-electrospinning device will be evaluated based on two product examples with industrial relevance, which are currently manufactured using the solvent electrospinning process. The aim is to show that the current production process can be advantageously replaced by melt electrospinning. The two examples considered here are nanofibers for air filtration, as well as nanofiber scaffolds as medical product.

Hardly any other topic has gained so much attention in Central Europe in the recent past as environmentally friendly road transport due to the high level of air pollution especially inside big cities. The biggest public debate was triggered by the "diesel scandal". Increased car exhaust emission levels have been observed in various diesel models. Highly efficient surface filters could be a key technology for solving this problem. An important feature of filter materials is their specific surface. Figure 6.2 shows the relationship between the specific surface area and the diameter of the fibers. As the fiber diameter increases, the specific surface area decreases. Conversely, the smaller the fiber, the larger the specific surface area. 


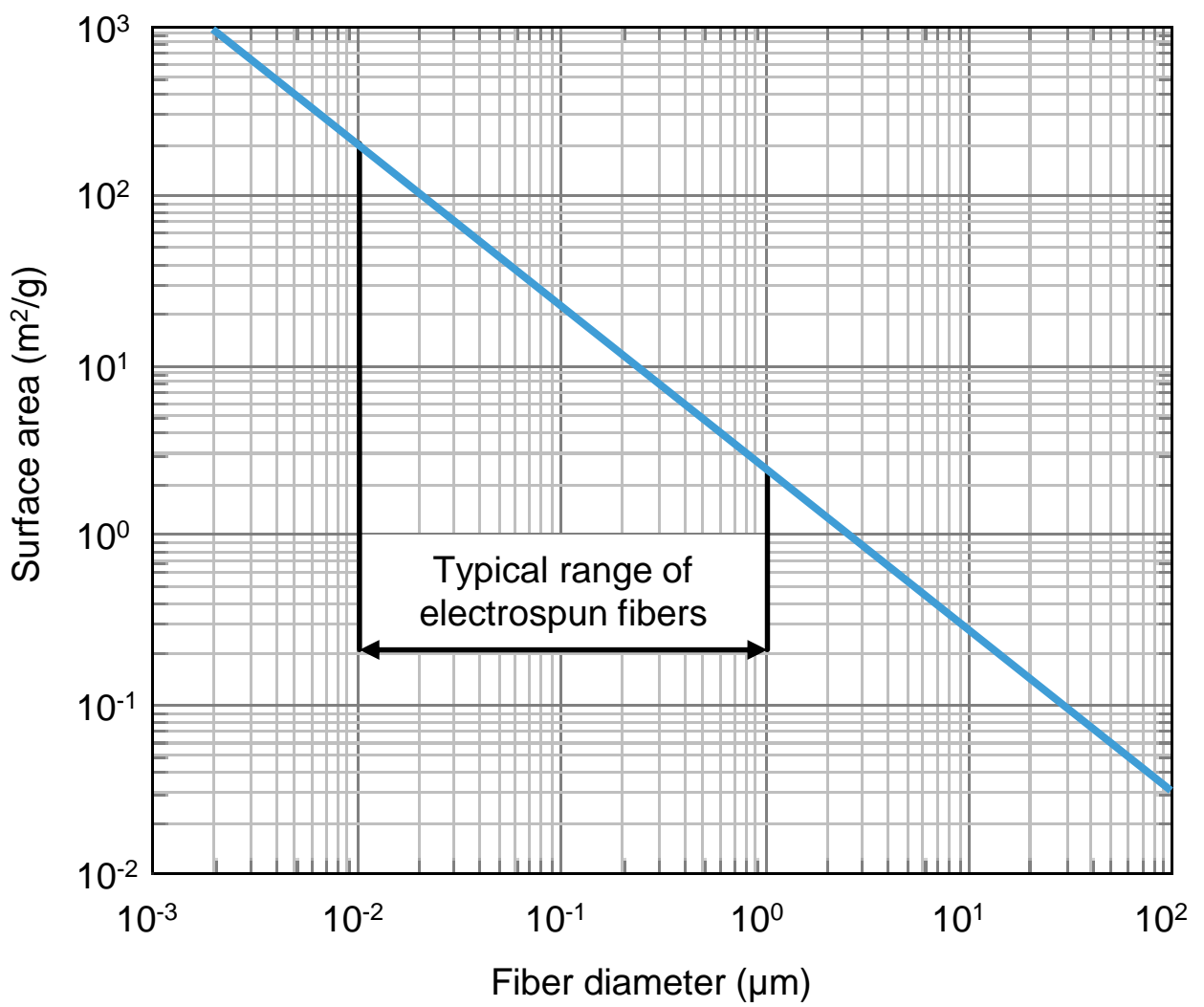

Fig. 6.2: Relationship between the specific surface area and the diameter of fibers.

Nanofibers with their large surface area per fiber mass and simultaneously high porosity improve filter performance and naturally occurring nanoparticles such as viruses and microscopic particles such as bacteria and fine dust can be filtered [8]. Already in 1965, Paul Dutch investigated the influence of fiber diameter on the ability to separate particles of different sizes in an air stream. It could be shown that the binding forces between fiber and dirt particles are always maximal when the diameters of fiber and particles are about the same size [9]. In addition to the separation efficiency, a filter medium is also characterized by its pressure drop. In the world of nanofibers and nanoparticles, physical effects occur which positively influence the flow resistance of these filter media. Considering the behaviour of air that can be observed while flowing around a microfiber, the flow velocity directly at the surface of the fiber is zero, which results in a correspondingly high flow resistance. For fibers with diameters smaller than $500 \mathrm{~nm}$, the so-called slip-flow effect occurs, which ensures that the flow velocity does not drop to zero at the surface for such small fibers, resulting in a comparatively low flow resistance [10]. This is of crucial importance for the filter industry, since instead of increasing flow resistance by increasing the surface area, flow resistance is reduced by increasing the surface area by using nanofibres.

MANN+HUMMEL (Ludwigsburg, Germany) developed nanofiber coated filter media for highly efficient particle separation, even of fine particles below one micrometer. These filter media are characterized by demonstrably higher separation efficiency with a high 
dust holding capacity. With diameters below one micrometer, these filter-coating fibers, produced using the solvent electrospinning process, are much smaller than the 10-30 $\mu \mathrm{m}$ thick carrier fibers (Figure 6.3) [11].

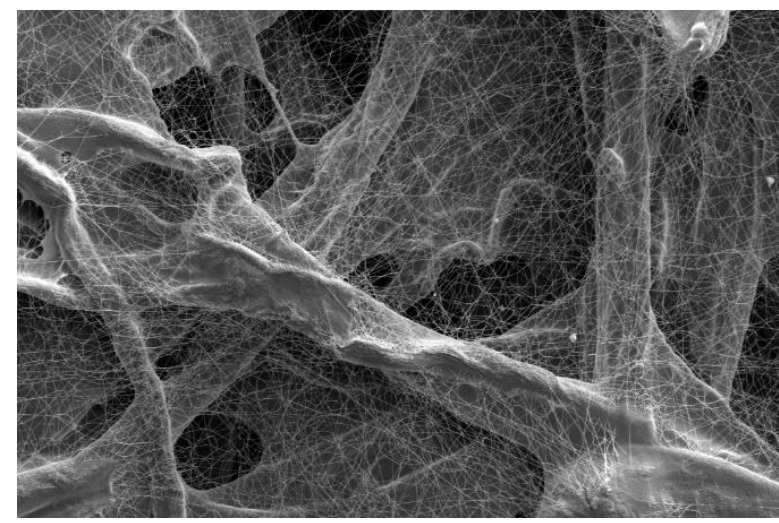

Fig. 6.3: MANN+Hummel filter-coating fibers on carrier material.

The comparison of the nanofiber filter medium with the pure carrier material shows the efficiency of nanofiber technology in filtering. In tests of filter elements according to standard ISO 5011, the nanofiber filter medium achieves up to 10 times less dust passage compared to the pure carrier material. Standard cellulose media enable the separation of $99.9 \%$ of the total mass of dust particles from the air. The close-meshed nanofiber layers increase the so-called separation efficiency to up to $99.99 \%$ [11]. Furthermore, the service life of such filter elements with their fabric-like nanofiber technology is two times longer than that of conventional filter media, which offers a considerable high potential for cost savings [8].

With our lowest average fiber diameter of $810 \mathrm{~nm}$ achieved using the 600 -nozzle meltelectrospinning device (Chapter 5), we meet the requirements for the fiber diameters of less than $1 \mu \mathrm{m}$ needed for the filter coating and can directly substitute the previously used manufacturing process. This is desirable from an economic and environmental point of view because only $2-10 \%$ of the liquid processed during solution electrospinning is the polymer (the rest is solvent that evaporates) whereas $100 \%$ of the processed liquid solidifies into fibers during melt electrospinning [1]. In Chapter 4 we were able to show that a process change from solution to melt electrospinning leads to a cost reduction of $99.7 \%$ in the first production cycle if only material costs are considered and no solvent recovery step is taken into account. The cost reduction results primarily from the absence of expensive solvents such as chloroform with a price of 1000 euro $/ \mathrm{kg}$. For the production of $1 \mathrm{~kg}$ of PLA fibers, $97 \%$ of the material costs can be saved from the second production cycle onwards compared to solution electrospinning with an estimated solution recovery of $90 \%$ [5].

Not only the filtration industry benefits from the use of nanofibers. Nanofibers from polylactic acid, an environmentally friendly and biodegradable polymer, are usually prepared by solvent electrospinning using dichloromethane, chloroform or $\mathrm{N}, \mathrm{N}$ dimethylformamide [2]. Besides the high cost for the solvents and the elaborate solvent 
recovery process, as described above, the potential carryover of toxic solvents into the final product raises an additional risk for biomedical applications (Chapter 4) [4].

DiPole Materials (Baltimore, United States) produces solution-electrospun BioPapers. BioPapers are nanofiber cell scaffolds composed of biologically-derived materials that create a cell microenvironment for drug screening, 3D bioprinting or tissue engineering [12]. Electrospun nanofiber nonwovens are structurally similar to the human extracellular matrix and thus promote successful cell growth [12]. Available are PLA scaffolds as $6 \mathrm{~mm}$ discs with a fiber diameter of approx. $650 \mathrm{~nm}$, randomly aligned with a pore size between 1-15 $\mu \mathrm{m}$. When changing for the melt-electrospinning process, a toxicity of the materials caused by solvent residues can be excluded [2]. The product required fiber diameters are a little smaller than the previously achieved smallest average fiber diameter of $810 \mathrm{~nm}$. Nevertheless, single fiber diameters of $420 \mathrm{~nm}$ have already been produced with the prototype melt-electrospinning device. Further increasing the temperature slightly and therewith reducing the material viscosity or reducing the spin pump speed can further reduce the average fiber diameter.

The increasing environmental awareness of the population and the resulting political goals are obliging the industry more and more to deal with the production of sustainable products. If existing product materials can be replaced by biobased polymers such as PLA, the production methods have also to be adapted sustainably. The presented upscaled melt-electrospinning process for the manufacture of microscale and nanoscale fibers prevents the use and disposal of toxic solvents, as well as a possible carry-over of the solvent into the final product, making a decisive contribution to a truly sustainable process chain. It could thus be shown that the device technology developed can be used advantageously compared to previous processes for the manufacture of actual industrially relevant product examples. Furthermore, the prototype melt-electrospinning device can serve as a model for a further scaling up in order to produce large-scale nonwovens with a width of more than actual $34 \mathrm{~cm}$. 


\section{References}

[1] Koenig K, Beukenberg K, Langensiepen F, Seide G. A new prototype meltelectrospinning device for the production of biobased thermoplastic submicrofibers and nanofibers. Biomaterials Research. 2019; 23(1):10.

[2] Koenig K, Hermanns S, Ellerkmann J, Saralidze K, Langensiepen F, Seide G. The effect of additives and process parameters on the pilot-scale manufacturing of polylactic acid sub-microfibers by melt electrospinning. Textile Research Journal. 2020:0040517520904019.

[3] Brown T, Dalton P, Hutmacher DW. Melt electrospinning today: an opportune time for an emerging polymer process. Elsevier. 2016; 56.

[4] Qin Y, Cheng L, Zhang Y, Chen X, Wang X, He X, et al. Efficient preparation of poly(lactic acid) nanofibers by melt differential electrospinning with addition of acetyl tributyl citrate. Journal of Applied Polymer Science. 2018; 135(31):46554.

[5] Koenig K, Balakrishnan N, Hermanns S, Langensiepen F, Seide G. Biobased Dyes as Conductive Additives to Reduce the Diameter of Polylactic Acid Fibers during Melt Electrospinning. Materials. 2020; 13(5):1055.

[6] Nayak R, Kyratzis IL, Truong YB, Padhye R, Arnold L. Melt-electrospinning of polypropylene with conductive additives. Journal of Materials Science. 2012; 47(17):6387-96.

[7] Zhou H, Green TB, Joo YL. The thermal effects on electrospinning of polylactic acid melts. Polymer (Guildf). 2006; 47.

[8] Chase GG, Swaminathan S, Raghavan B, Wei Q. 7 - Functional nanofibers for filtration applications. Functional Nanofibers and their Applications: Woodhead Publishing; 2012. p. 121-52.

[9] National Air Filtration Association: NAFA GUIDE TO AIR FILTRATION.2007.

[10] Graham K, Ouyang, M., Reather, T., Grafe, T., McDonald, B., Knauf, P. Donaldson Co. Inc . Polymeric Nanofibers in Air Filtration Applications. Annual Technical Conference \& Expo of the American Filtration and Separation Society; Galveston, Texas, 9-12 April 2002.

[11] Mann+Hummel. Nanofaserfiltermedien für Motoransaugluftfiltration. https://www.mann-hummel.com/de/forschung-und-entwicklung/filtertechnik-imdetail/nanofaserfiltermedien-fuer-motoransaugluftfiltration/ (accessed 26 February 2020).

[12] DiPole Materials. 3D NANOFIBER SCAFFOLDS. https://www.dipolematerials.com/wp/content/uploads/2018/10/DiPole_Materials_ 3D-Nanofiber-Scaffolds.pdf (accessed 26 February 2020). 



\section{Chapter 7}

Summary and outlook

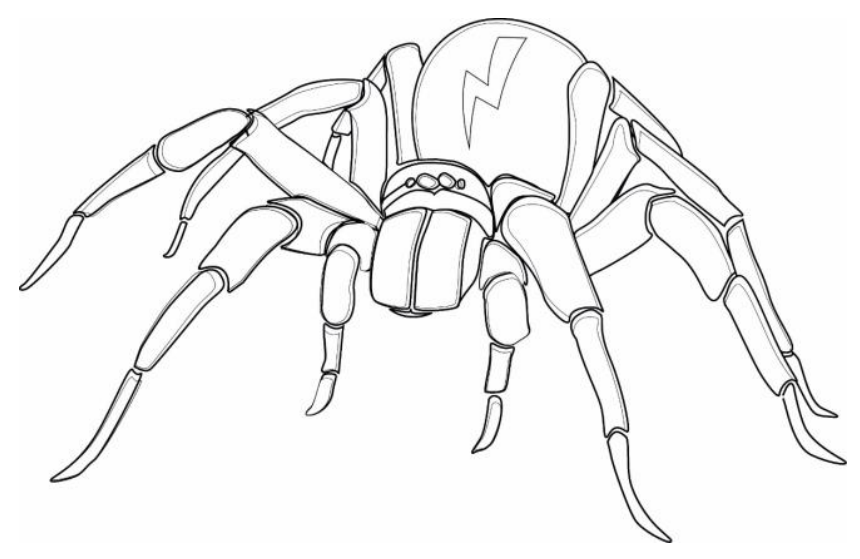




\section{Summary}

In this thesis, a new developed scaled-up melt-electrospinning prototype featuring 600 nozzles, bridging the gap between laboratory-scale and industrial-scale nanofiber manufacturing, is presented (Chapter 1). Herewith tackling the industry-relevant key challenge of increasing the production of nanofibers for various applications such as filtration and medicine in a sustainable and efficient way. The prototype device vastly exceeds the capabilities of any state-of-the-art technology and is successfully used to produce nanofibers with comparable quality and properties to nanofibers produced with the more conventional solution electrospinning process.

Chapter 2 focuses on the forces acting on the polymer melt, process parameters and electrospinning setups influencing the fiber formation. Based on the technical principles, the following assumptions are made for this work: The collector of the pilot-scale meltelectrospinning device is positively charged and the spinneret is grounded, the distance between the collector and the spinneret $(11 \mathrm{~cm})$, as well as the applied voltage $(60 \mathrm{kV})$ are kept constant for all trials. Hence, the influence of the parameters polymer flow rate and temperature on the fiber diameter as well as material modifications resulting in an increased electrical polymer conductivity and/or decreased polymer viscosity can be investigated and validated uninfluenced by these parameters.

In Chapter 3, the feasibility of fabricating polypropylene nanofibers is investigated using the conductive additives sodium stearate, sodium oleate and Irgastat during melt electrospinning with a single-nozzle lab-scale and the 600-nozzle pilot-scale device. A comparison is made between the two devices with regard to the effects on the fiber diameter and the material behavior. The experiments have shown that a transfer of the results from the laboratory- to the pilot-scale melt-electrospinning device is not possible without further ado. The major issues are the dwell time in the spinneret compared to the syringe and the different shear rates. The high melt flow PP HL712FB cannot be processed with the pilot-scale device due to its low viscosity, resulting in an insufficient pressure built up within the spinneret, which leads to droplet formation instead of a continuous fiber formation. The additives - in particular sodium oleate - lead to a strong degradation of the polymer, which requires extensive cleaning of the devices from degradation products and impairs the formation of fibers. The integration of a climate chamber leads to the finest fibers on a laboratory-scale with a fiber diameter of less than $500 \mathrm{~nm}$ using the material combination PP HL712FB + 4\% (w/w) NaSt and 2\% (w/w) Irgastat. At the pilot scale, the smallest fiber diameter of $6.64 \mu \mathrm{m}$ is achieved with PP HL508FB $+2 \%(w / w)$ NaSt, which yields a fiber diameter of $2.07 \mu \mathrm{m}$ on a laboratory scale.

In Chapter 4, the manufacturing of biobased polylactic acid sub-microfibers by melt electrospinning using a single-nozzle laboratory-scale and the novel 600-nozzle pilotscale device combined with the conductive and viscosity-reducing additives: sodium stearate, sodium chloride and a polyester-based plasticizer is investigated. Additionally, 
a two-way variance analysis (ANOVA) is performed to determine the influence of the independent parameters spin pump speed, temperature and additive concentration on the fiber diameter. The first PLA-based fibers in the low micrometer range are sucessfully produced. The addition of $6 \%(\mathrm{w} / \mathrm{w}) \mathrm{NaSt}$ is required to produce the smallest average fiber diameter $(3.77 \mu \mathrm{m})$ using a spinneret temperature of $195^{\circ} \mathrm{C}$ and a spin pump speed of $0.5 \mathrm{rpm}\left(0.16 \mathrm{~cm}^{3}\right)$. The smallest single fiber diameter $(1.23 \mu \mathrm{m})$ is achieved at the same conditions but using a spin pump speed of $2 \mathrm{rpm}\left(0.64 \mathrm{~cm}^{3}\right)$. A higher quantity of additive promotes material degradation due to the processing and dwell time, which inhibited fiber formation. Fibers with diameters in the low micrometer range are achieved, comparable with previous reports, without the integration of a heated spinning chamber or additional air flow, which are already widely used at the laboratory scale to achieve further stretching of the fibers. The carried out ANOVA reveals statistical significance for all examined, independent factors (spin pump speed, additive concentration and temperature). Evaluated by the effect strength $\eta$, the additive concentration has the greatest influence on the fiber diameter. At the laboratory scale, the effect of viscosityreducing additives ( $\mathrm{NaSt}$ and plasticizer) as well as $\mathrm{NaCl}$, which increases the conductivity of the polymer melt, are determined. The smallest average fiber diameter $(16.44 \mu \mathrm{m})$ is achieved by adding $2 \%(\mathrm{w} / \mathrm{w}) \mathrm{NaCl}$ but the spinning process cannot be stabilized. The formation of a Taylor cone followed by fiber deposition is achieved with compounds containing NaSt or plasticizer, but the latter does not reduce the viscosity of the melt at the concentrations tested and its influence on fiber diameter therefore cannot be determined. Concluded is that the additive NaSt has the greatest potential to optimize the material properties of PLA for melt electrospinning.

Since the added salts can potentially increase the hydrolysis and degradation of the moisture-sensitive PLA, the use of the biobased dyes alizarin, hematoxylin and quercetin as conductivity-enhancing additives and a biobased plasticizer in laboratory-scale melt electrospinning is also investigated in Chapter 4. All dyes and dye/plasticizer combinations contribute to the desirable reduction of fiber diameter compared to pure melt-electrospun PLA fibers, which facilitates the development of an economical and environmentally friendly process for the production of microfibers and nanofibers that could ultimately replace solution electrospinning. The formation of a Taylor cone followed by continuous fiber deposition is observed for all dyes and dye/plasticizer combinations. The finest fibers (16.04 $\mu \mathrm{m}$ in diameter) are produced by adding $2 \%(\mathrm{w} / \mathrm{w})$ hematoxylin, reducing the average fiber diameter by $77 \%$ compared to pure PLA. However, hematoxylin induces polymer degradation at a spinning temperature of $275^{\circ} \mathrm{C}$, which reduces the $M_{w}$ and, therefore, favors the production of finer fibers. The addition of alizarin produces finer fibers than pure PLA despite the increase in melt viscosity, indicating that alizarin has a profound effect on the electrical conductivity of the melt. $A$ combination of alizarin (to increase conductivity) and a plasticizer (to reduce viscosity) reduced the fiber diameter to $23.80 \mu \mathrm{m}$, which is $63 \%$ narrower than the pure PLA fibers. The addition of quercetin reduced the melt viscosity but had a limited effect on electrical conductivity compared to alizarin, and the finest fibers containing this additive (achieved 
by adding $2 \%(\mathrm{w} / \mathrm{w})$ liquid quercetin) were $36.72 \mu \mathrm{m}$ in diameter. The comparison of fibers produced by melt spinning, melt spinning with post-drawing, and melt electrospinning reveals that the melt-electrospun fibers had a similar degree of crystallinity to partially-oriented filaments and are not comparable to drawn filaments.

In previous experiments, using the pilot-scale melt-electrospinning device without appropriate climate control, fibers exceeding $1 \mu \mathrm{m}$ in diameter are produced, because the drawing of fibers is inhibited by the rapid cooling of the polymer melt. In Chapter $\mathbf{5}$ the effects resulting of an integration of a climate control system in form of a glass chamber are investigated. The glass chamber creates a temperature gradient exceeding the glass transition temperature of the polymer, allowing the further drawing of fibers below the spinneret. An average fiber diameter of $810 \mathrm{~nm}$ is achieved using PLA Ingeo Biopolymer 6252 (Figure 7.1), and the finest individual fiber (420 nm in diameter) was produced at a spin pump speed of $5 \mathrm{rpm}$ and a spinneret set temperature of $230^{\circ} \mathrm{C}$. The fiber diameters achieved are the smallest fiber diameters yet achieved with an upscaled multi-nozzle melt-electrospinning device of this size.

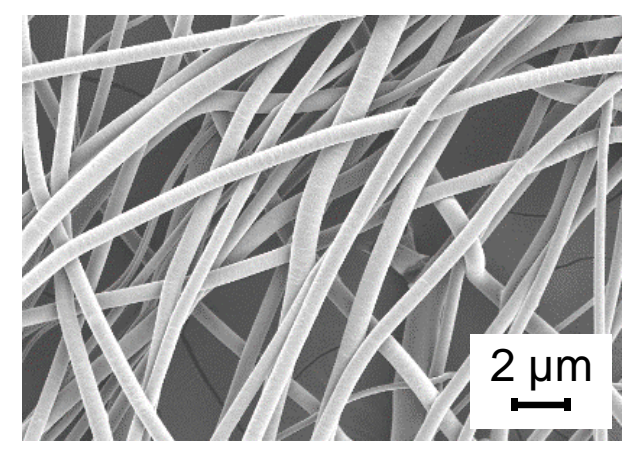

Fig. 7.1: PLA Ingeo Biopolymer 6252 fibers with an average fiber diameter of $810 \mathrm{~nm}$.

The presented upscaled melt-electrospinning process for the manufacture of microscale and nanoscale fibers prevents the use and disposal of toxic solvents, as well as a possible carry-over of the solvent into the final product, making a decisive contribution to a truly sustainable process chain. With the valorization (Chapter 6) it is shown that the device technology developed can be used advantageously compared to previous processes for the manufacturing of actual industrially relevant product examples such as filtration nonwovens and medical tissues.

\section{Outlook}

There are still opportunities for further improvement of the performance of the device and material. In future attempts a combination of the two salts, $\mathrm{NaCl}$ and $\mathrm{NaSt}$, might also be tested in order to combine the respective positive effects of increasing conductivity and lowering viscosity. Thus, lowering the fiber diameter even further. The experiments with PLA and alizarin should be transferred to pilot scale, as they have shown promising 
results on laboratory scale. Other polymers such as the biobased, biodegradable polybutylene succinate seem promising to be tested in the melt-electrospinning process. Preliminary tests have shown that the fiber diameter can be halved compared to pure PLA using the same process conditions in laboratory scale. Regarding the thermal degradation problem, the spinneret has to be optimized to reduce dwell times. Furthermore, individually controlled collector tips in a multi-nozzle structure with the writing ability of melt electrospinning could lead to the development of truly innovative microfiber and nanofiber products. 



\section{Appendix}

List of publications

Curriculum vitae

Additional figures

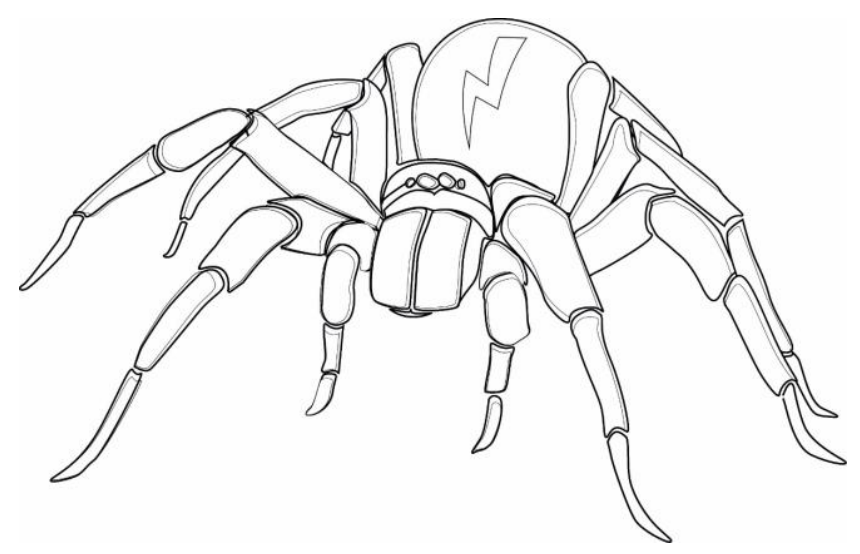




\section{List of publications}

\section{Journal articles}

Koenig K, Beukenberg K, Langensiepen F, Seide G. A new prototype meltelectrospinning device for the production of biobased thermoplastic sub-microfibers and nanofibers. Biomaterials Research. 2019; 23(1):10.

Koenig K, Daenicke J, Langensiepen F, Seide G, Schubert DW. From lab to pilot scale: Melt-electrospun nanofibers of polypropylene with conductive additives. J Nanomater Mol Nanotechnol. 2019; 8(2).

Koenig K, Hermanns S, Ellerkmann J, Saralidze K, Langensiepen F, Seide G. The effect of additives and process parameters on the pilot-scale manufacturing of polylactic acid sub-microfibers by melt electrospinning. Textile Research Journal. 2020:0040517520904019.

Koenig K, Balakrishnan N, Hermanns S, Langensiepen F, Seide G. Biobased Dyes as Conductive Additives to Reduce the Diameter of Polylactic Acid Fibers during Melt Electrospinning. Materials. 2020; 13(5):1055.

Koenig K, Langensiepen F, Seide G. Pilot-scale production of polylactic acid nanofibers by melt electrospinning. e-Polymers. 2020; 20: 233-241.

\section{Magazine articles}

Koenig K., Manvi P, Gries T. Star-Tex: Stärke, ein kostengünstiger Rohstoff für die Textilindustrie. Textile Network 2018. https://textile-network.de/de/ForschungEntwicklung/Institut-fuer-Textiltechnik-an-der-RWTH-Aachen/Star-Tex-Staerke-einkostenguenstiger-Rohstoff-fuer-die-Textilindustrie. *

\section{Reports}

Koenig $\mathrm{K}$, Langensiepen $\mathrm{F}$. Commercially available fibers based on renewable raw material and fiber potentials of bioplastics. The Fiber Year: Word Survey on Textiles \& Nonwovens 2018: 18, pp. 66-69.

\section{Poster presentations}

Koenig K, Hermanns S, Ellerkmann J, Langensiepen F, Seide G. Melt electrospinning from lab to pilot scale: Challenges, solution approaches and applications. Aachen, Dresden and Denkendorf International Textile Conference 2018, Aachen, Germany.

Koenig K, Hermanns S, Ellerkmann J, Langensiepen F, Seide G. Nanotechnology for the automotive sector: Upscaling of the melt-electrospinning process for an economic production of efficient, biobased filter systems. Material, Science and Engineering Congress 2018, Darmstadt, Germany. 
Koenig K, Manvi P, Gries T. Star-Tex: Stärkebasierte Textilien - Kostengünstige Textilien aus Biopolymeren. FNR Gemeinschaftsstand, Hannover Messe 2018, Hannover, Germany.*

\section{Oral presentations}

Koenig K, Kammler S. GraSage: Modelling of the electrical and thermal transport mechanisms in graphene nano-modified polymer compounds and fibres. Aachen, Dresden and Denkendorf International Textile Conference 2019, Dresden Germany. *

Koenig K. Introducing GraSage. Institute of Data Science. UM Data Science Research Seminar (23 May 2019), Geleen, The Netherlands. *

Koenig K. Introducing GraSage. Graphene Flagship Partnering Division Meeting, Graphene Week 2019, Helsinki, Finland.*

*The publication does not constitute a part of this work. 


\section{Curriculum vitae}

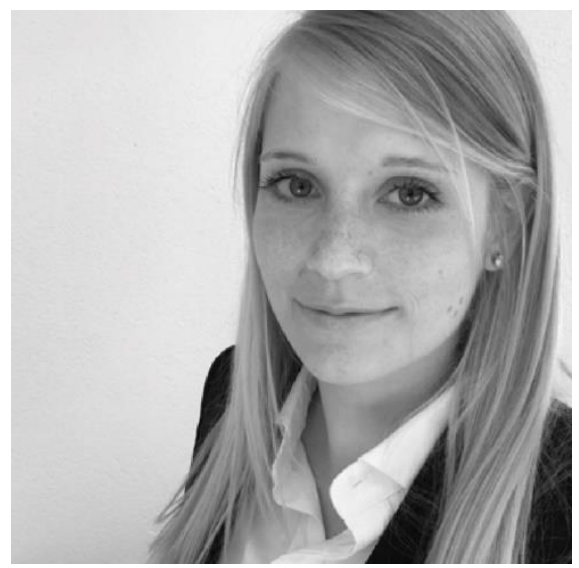

Kylie Koenig was born in Cologne (Germany) on June 29, 1990. Kylie graduated from Freiherr-vomStein Gymnasium (Roesrath, Germany) in 2009. From 2009-2013, Kylie followed her Bachelor studies in aerospace engineering at the University of Stuttgart (Germany). After obtaining her Bachelor degree, she continued her studies in aerospace engineering at RWTH Aachen University (Germany), graduating with a Master degree in 2016. Before graduating, she completed a five-month internship at Airbus (Bremen, Germany) in the composites department to deepen her knowledge of fiber technologies. Kylie completed her master thesis on the topic "Miscibility and refractive index study of polymer blends to produce a transparent fiber-reinforced plastic" at the Institute of Textile Technology (ITA) of RWTH University. In January 2017, she started working as a research assistant at the ITA in the Biopolymer Research Group of Prof. Dr.-Ing. habil. Dipl.-Wirt. Ing. G.H. (Gunnar) Seide with the aim of obtaining a doctorate degree. Since July 2018, she has been continuing her work the newly founded cooperation institute AMIBM of Maastricht University (Aachen-Maastricht Institute for Biobased Materials) (Geleen, The Netherlands). Kylie has gained extensive experience in the extrusion of biobased multifilament yarns and the development of novel spinning processes. The focus of her $\mathrm{PhD}$ study is on the development of an upscaled melt-electrospinning process for an industry-related production of nanofibers. The scope of her study included the development and adaptation of the required device technology as well as the investigation of melt-electrospinable materials and relevant parameters influencing the spinning process. In addition to researching for her doctorate, Kylie has taken the project and management lead for three public funded projects: ZIM BioKombiGarn - Biobased wrapping yarn, FNR StarTex-Cost-effective textiles made from starch and NWO GraSage - Graphene modified electrically conductive fibers. She has presented the projects and contents of her doctoral research in journal articles, at fairs, congresses and workshops, as well as to industry partners. Kylie has also worked on various direct industry research projects, has actively been involved in project acquisition and supervised several student work projects. 


\section{Additional figures}

\section{Chapter 4}

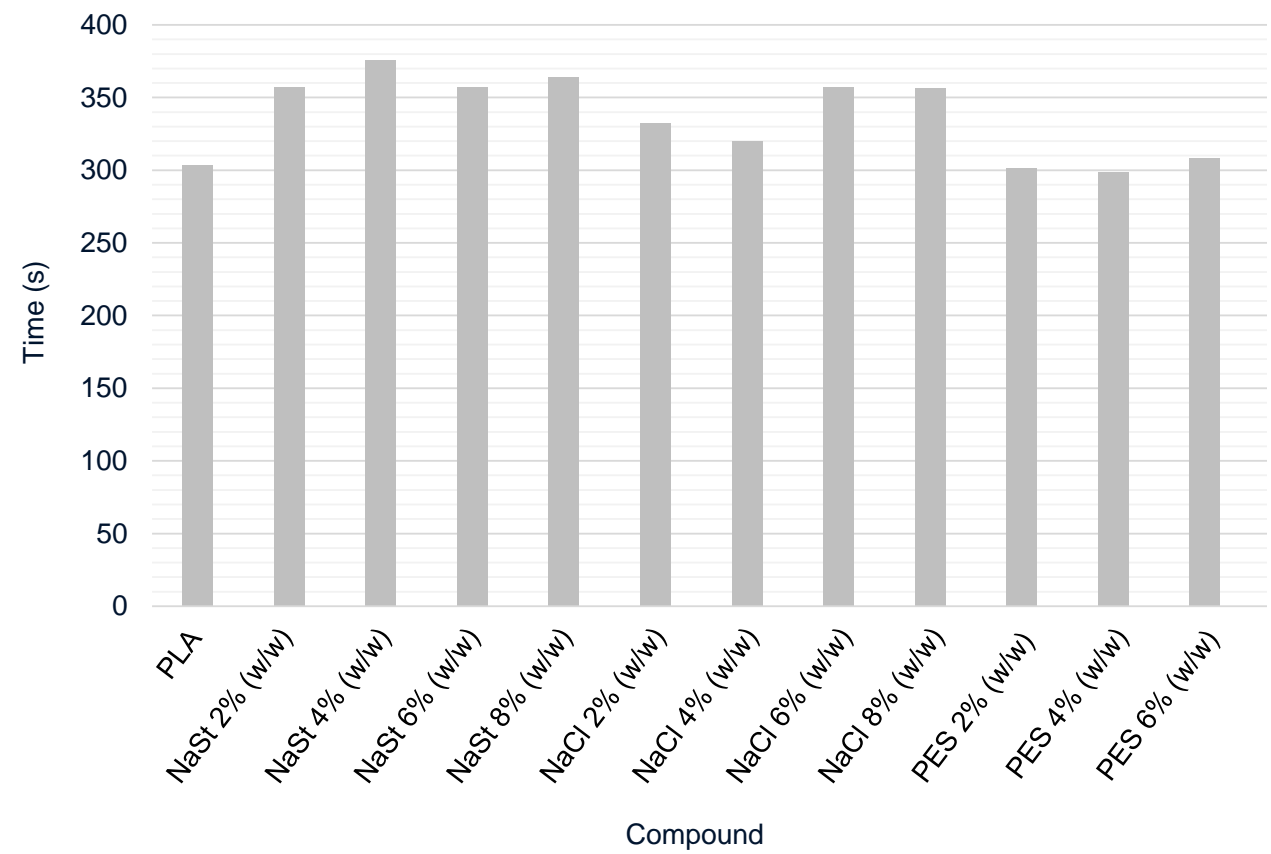

Fig. A1: Time until the point of complex viscosity is captured for a frequency of $10 \mathrm{rad} / \mathrm{s}$.

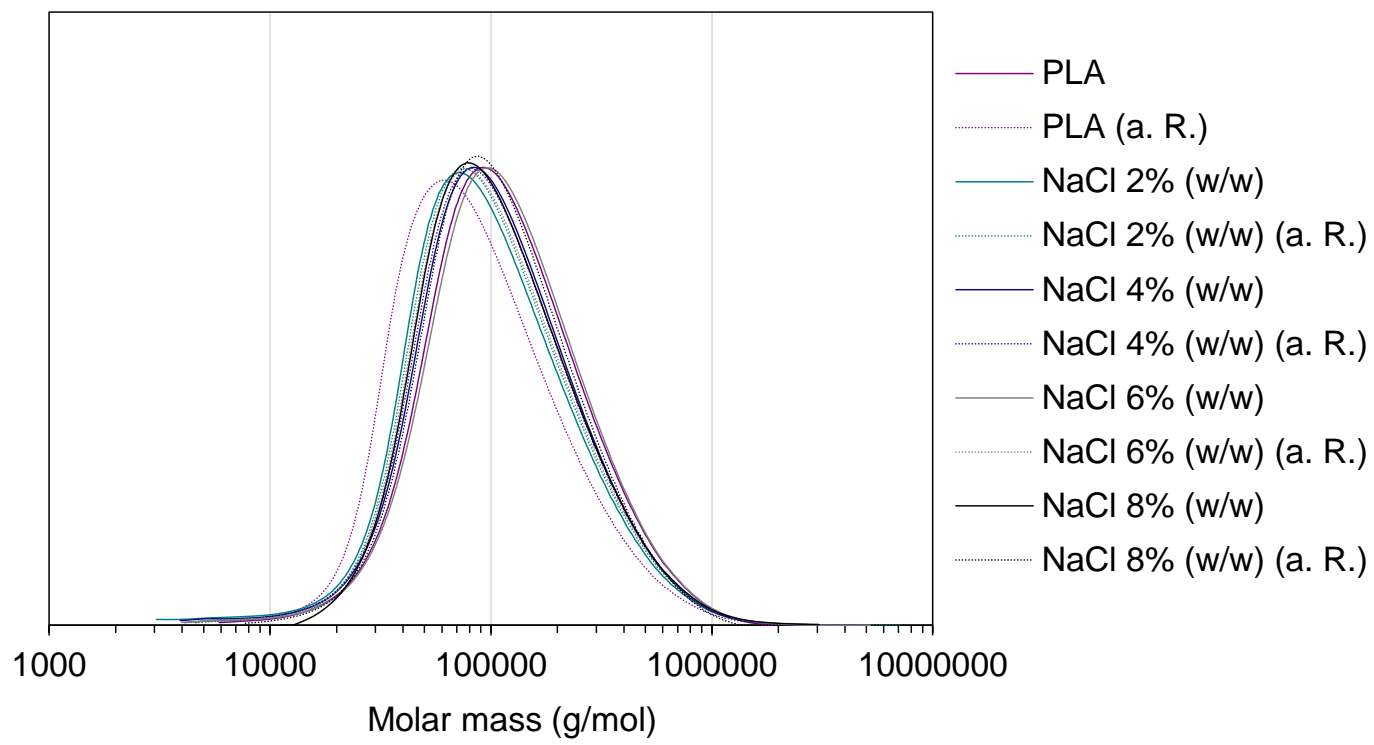

Fig. A2: GPC curves of virgin PLA and PLA containing 2-8\% (w/w) NaCl after compounding and after the time influence of the rheometer test (a.R.). 


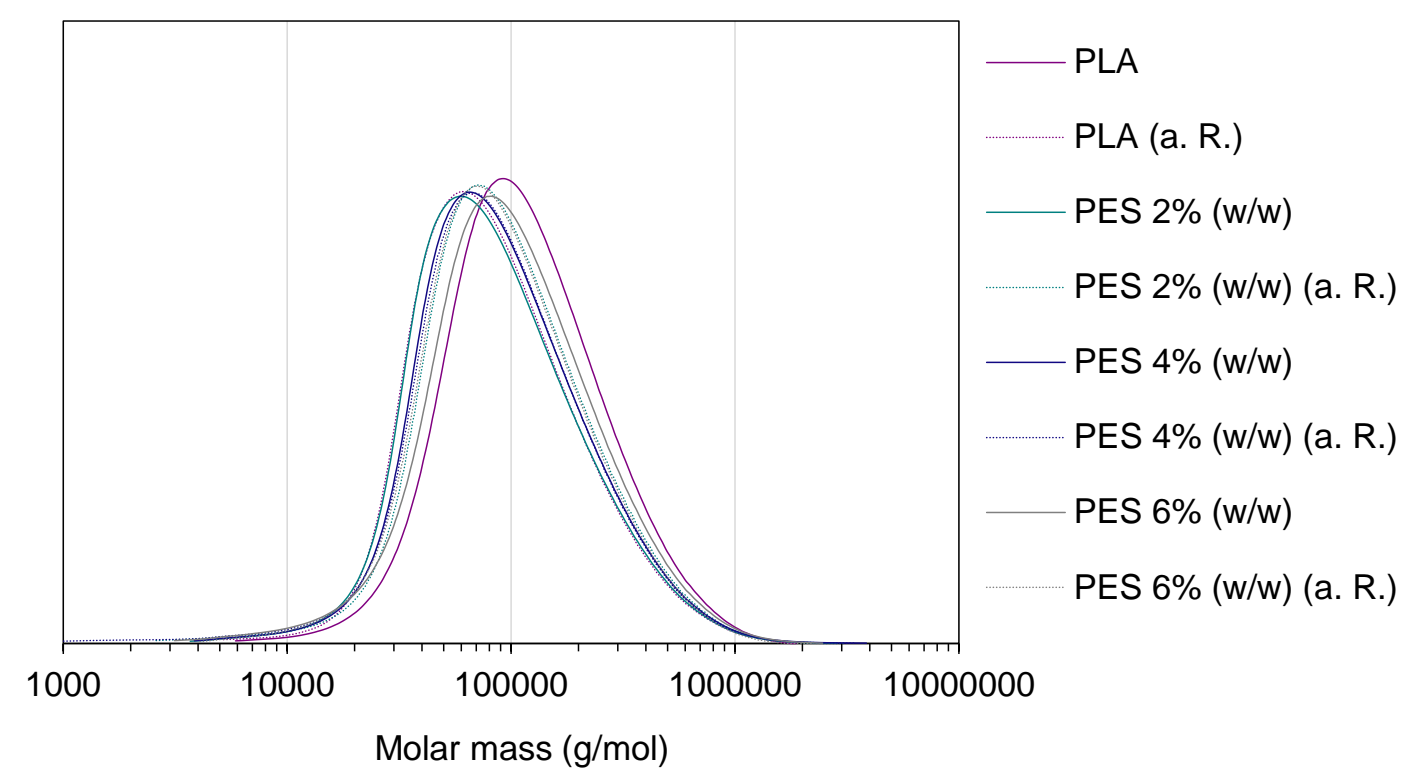

Fig. A3: GPC curves of virgin PLA and PLA containing 2-6\% (w/w) PES after compounding and after the time influence of the rheometer test (a.R.).

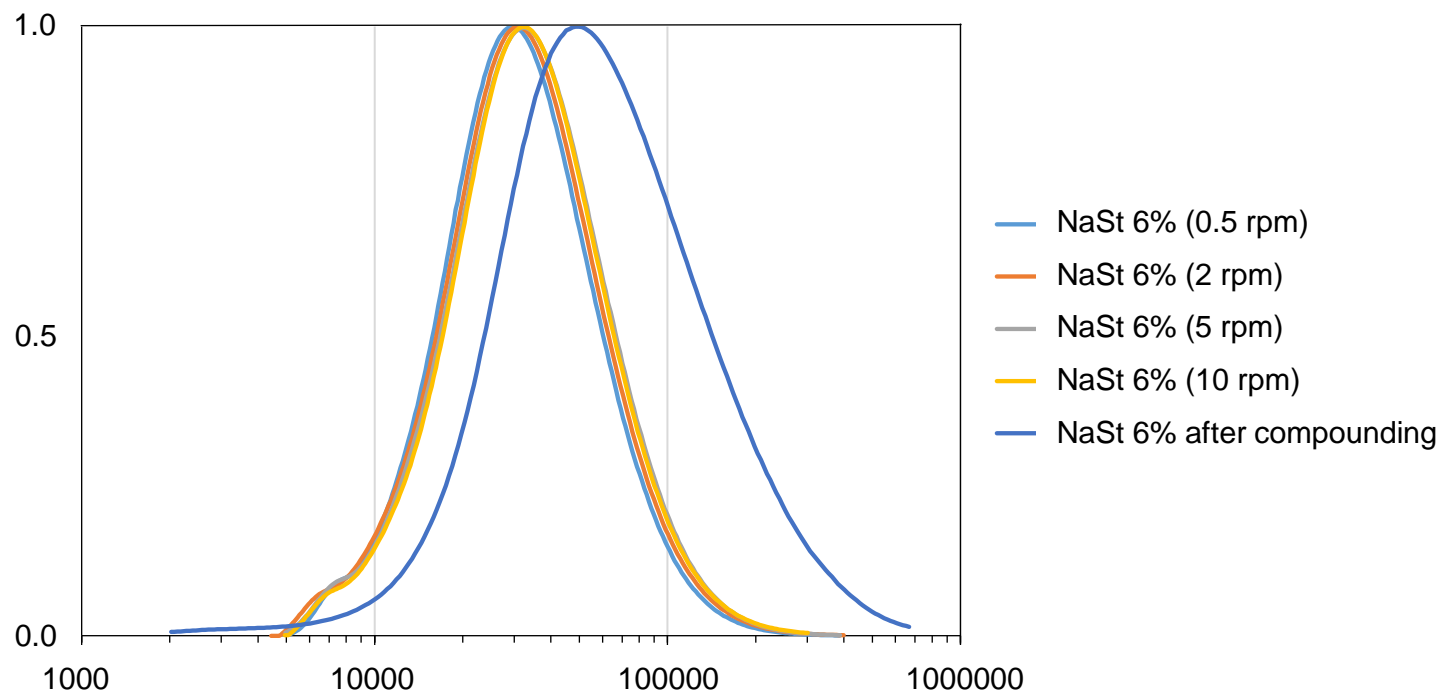

Fig. A4: GPC curves of PLA with $6 \%(\mathrm{w} / \mathrm{w})$ NaSt after compounding and after melt electrospinning with spin pump speeds from 10-0.5 rpm at a temperature of $205^{\circ} \mathrm{C}$ (normalized to 1 ). 


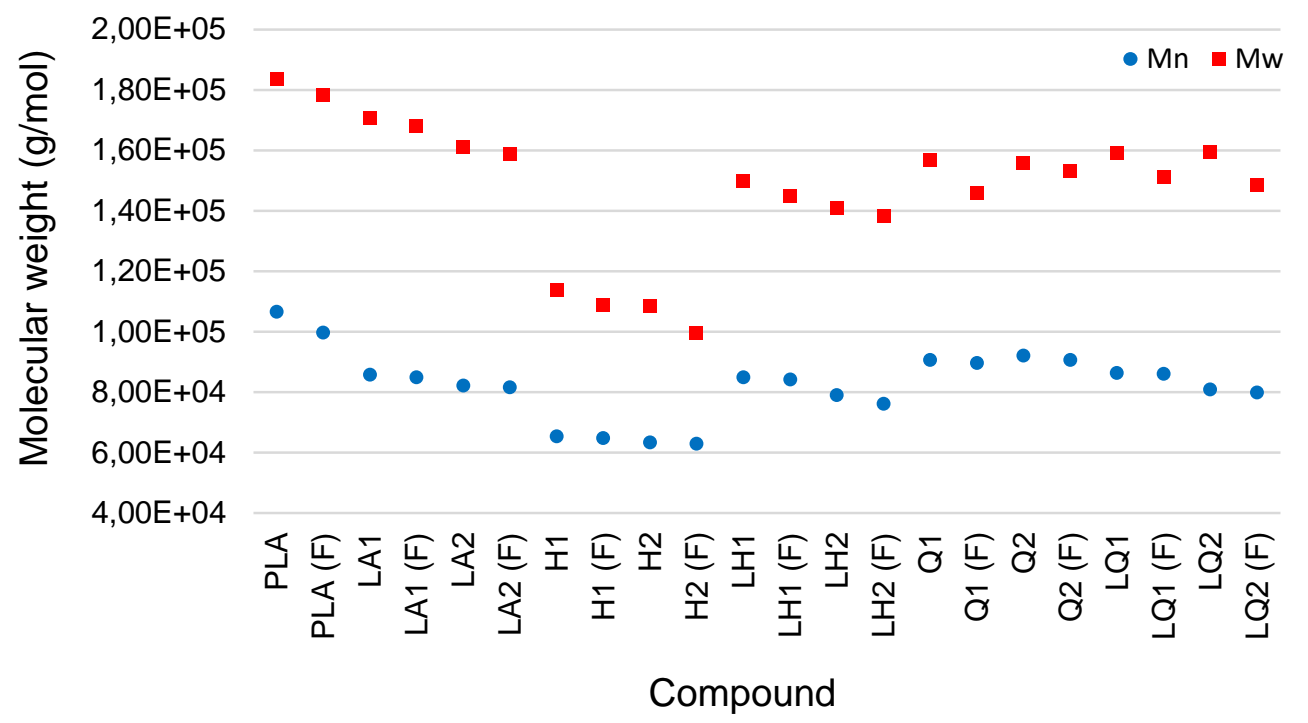

Fig. A5: Molecular weight of PLA, PLA/dye compounds and the melt-electrospun fibers $(F)$. 
It took engineering genius and hard work.

It took teamwork and individual skill.

It took courage and sacrifice.

Not everyone can explore space. But we all have our own moons to reach for. If you set your sights high, you may accomplish more than you ever dreamed was possible.

- Buzz Aldrin -

Reaching For The Moon, 2005 